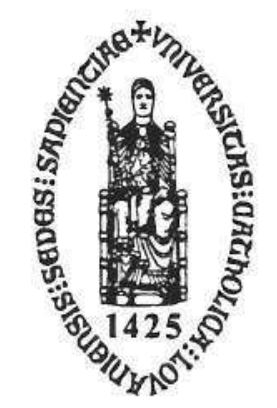

\author{
K.U. LEUVEN
}

Faculteit Der Wetenschappen

\title{
Curves and Surfaces in Minkowski Space
}

\author{
Johan Walrave \\ Doctoraatsverhandeling
}

Promotor : Prof. Dr. L. Verstraelen

Co-Promotor : Prof. Dr. I. Van de Woestyne 
Graag zou ik iedereen die bijgedragen heeft tot het voltooien van deze verhandeling willen bedanken.

Enkele mensen wil ik toch uitdrukkelijk bedanken. Ten eerste zou ik mijn promotor Prof. Dr. L. Verstraelen willen bedanken voor zijn vertrouwen in mij en voor zijn vele waardevolle raadgevingen over de jaren heen. Ten tweede wil ik mijn co-promotor Prof. Dr. I. Van de Woestyne bedanken. Zijn deur stond immers altijd voor mij open en als ik een probleem had, van welke aard ook, dan kon - en kan - ik altijd bij hem terecht. Ten slotte gaat mijn dank ook uit naar Prof. Dr. F. Dillen, Prof. Dr. I. Mihai en Prof. Dr. L. Vrancken voor hun vele tips en aanwijzingen die voor mij van groot belang waren.

Ook de mensen van de K.U.Brussel wil ik bedanken omdat zij mij de mogelijkheid geboden hebben om aan dit doctoraat te werken; in het bijzonder Pro-Rector Prof. Dr. F. Vanhemelryck, Rector Prof. Dr. F. Gotzen, de vorige Decaan van de F.E.T.E.W. Prof. Dr. E. Raymaekers, de huidige Decaan en mijn titularis Statistiek Prof. Dr. J. Degadt, mijn titularis Wiskunde Prof. Dr. R. Donckels, alle collega's en alle studenten van de voorbije vijf jaren.

Mijn laatste woorden van dank gaan naar mijn naaste familieleden, vooral mijn ouders en mijn vrouw Greet wil ik in de bloemetjes zetten. Hun bijdrage is van een onschatbare waarde. Sommige mensen zijn immers gewoon belangrijk omdat ze er zijn. 


\section{CONTENTS}

\section{Preface}

Chapter I. Curves in Minkowski Space 1

$\S 1$ Introduction 1

$\S 2$. The Moving Frenet Frame 4

§3. Curves with constant curvature 14

§4. Curves of restricted type $\quad 23$

$\S 5$. Curves of finite type $\quad 31$

Chapter II. Surfaces in Minkowski Space 42

§1.Introduction $\quad 42$

§2.Minimal surfaces $\quad 45$

$\S 3$. Ruled surfaces of finite type $\quad 53$

§4.Ruled surfaces of constant mean curvature $\quad 62$

$\begin{array}{ll}\text { Chapter III. Tensor product surfaces } & 69\end{array}$

§1.Introduction $\quad 69$

$\begin{array}{ll}\text { §2. Minimal tensor product surfaces } & 76\end{array}$

§3. Totally real and complex tensor product surfaces $\quad 82$

$\S 4$.Pseudo-minimal tensor product surfaces $\quad 84$

$\S 5$.Pseudo-umbilical tensor product surfaces $\quad 88$

$\begin{array}{lr}\text { Pictures } & 91\end{array}$

$\begin{array}{lr}\text { References } & \mathbf{1 1 0}\end{array}$

$\begin{array}{ll}\text { Beknopte samenvatting } & 113\end{array}$ 


\section{PREFACE}

A forerunner in the study of the differential geometry of curves in the Euclidean plane was Huygens, although at the time when he undertook his work on this subject, he couldn't dispose of the calculus. Yet, when investigating some problems related to pendulums and clocks, he was led to the notion of curvature of plane curves at a point $p$ of the curve. The first one who obtained a proper formula for the curvature in terms of the derivatives of the curve $\alpha$ was Newton in his work on the method of fluxions of which a manuscript dates at least from 1671. Newton considered a curve $\alpha(t)=\left(\alpha_{1}(t), \alpha_{2}(t)\right)$ as the trajectory described by a point moving around in the plane in function of the time $t$. He defined the curvature as follows. On the curve, take two nearly points $q_{1}$ and $q_{2}$, one at each side of a given point $p$. These three points together generically determine a circle with center $m$. In the limit, when both $q_{1}$ and $q_{2}$ go to $p$ along the curve $\alpha$, one obtains a special circle, which is tangent to $\alpha$ at $p$ (i.e. at $p, \alpha$ and this circle have the same tangent line ), and which among all such tangent circles has the closest contact with $\alpha$ at $p$. This circle is called the osculating (or kissing) circle of $\alpha$ at $p$. Let its center be $c$ and its radius $r$. Newton called $c$ the center of curvature, $r$ the radius of curvature and $\kappa=\frac{1}{r}$ the curvature of the curve $\alpha$ at the point $p$. He explicitely computed $\kappa$ to be given by

$$
\kappa=\frac{\dot{\alpha}_{1} \ddot{\alpha}_{2}-\ddot{\alpha}_{1} \dot{\alpha}_{2}}{\left(\dot{\alpha}_{1}^{2}+\dot{\alpha}_{2}^{2}\right)^{\frac{3}{2}}} .
$$

Instead of working with an arbitrary parameter $t$, the arclength parameter $s$ can be used, such that $\dot{\alpha}_{1}^{2}+\dot{\alpha}_{2}^{2}=1$. Define $T(s)$ to be the unit tangent vector of $\alpha$, and $N(s)$, the unit normal at $s$, to be the unit vector such that $N(s)$ is perpendicular to $T(s)$, and $\{T(s), N(s)\}$ is the standard orientation of $\mathbb{R}^{2}$. The curvature $\kappa(s)$ of $\alpha$ at $s$ is then defined by the equation

$$
\dot{T}(s)=\kappa(s) N(s) \text {. }
$$

Notice that $|\kappa(s)|=|\ddot{\alpha}(s)|$ is the length of $\ddot{\alpha}(s)$, which is perpendicular to the unit vector $\dot{\alpha}(s)$, clearly $|\kappa(s)|$ is also the area of the rectangle spanned by $\dot{\alpha}(s)$ and $\ddot{\alpha}(s)$. This area is given by $\operatorname{det}(\dot{\alpha}(s), \ddot{\alpha}(s))$, so

$$
\kappa(s)=\left|\begin{array}{cc}
\dot{\alpha}_{1}(s) & \ddot{\alpha}_{1}(s) \\
\dot{\alpha}_{2}(s) & \ddot{\alpha}_{2}(s)
\end{array}\right|=\dot{\alpha}_{1} \ddot{\alpha}_{2}-\ddot{\alpha}_{1} \dot{\alpha}_{2},
$$

which corresponds with the definition given by Newton. The fundamental, or congruence, theorem of Euclidean planar curves states that, up to Euclidean motions, given a continuous function $\kappa$ with variable $s$, there exist a unique curve $\alpha$ in $\mathbb{E}^{2}$ with $s$ as arclength parameter and such that $\kappa(s)$ is the curvature of $\alpha$ at $\alpha(s)$. This means that up to rotations and translations, a curve in $\mathbb{E}^{2}$ is completely charachterized by its curvature. 
For the study of the differential geometry of space curves in $\mathbb{E}^{3}$, the great step forward came with the Frenet-Serret formulas. They were obtained independently by Frenet in 1847 and by Serret in 1851. First they defined the orthonormal frame $\{T, N, B\}$ - known as the Frenet frame - along $\alpha$ a space curve parameterized by the arclength parameter $s . T$ is the velocity or unit tangent vector field of $\alpha$. The acceleration $\ddot{\alpha}(s)$ of the curve $\alpha$ at the point $\alpha(s)$ is a vector which stands perpendicular to $T(s)$. If $\ddot{\alpha}(s) \neq 0$, the principal normal vector field $N$ is the normalized acceleration field $\ddot{\alpha}$. The binormal vector field $B$ is determined by the vectorial product of $T$ and $N$. The Frenet-Serret formulas are then given by

$$
\begin{aligned}
\dot{T} & =\kappa N \\
\dot{N} & =-\kappa T+\tau B \\
\dot{B} & =-\tau N
\end{aligned}
$$

and determine all successive derivatives of $\alpha$. The functions $\kappa$ and $\tau$, which are respectively determined by the first and third formula, are called the first and second curvatures of $\alpha$, or the curvature and the torsion. The fundamental theorem of Euclidean space curves states that if $\kappa$ and $\tau$ are two continuous functions of $s$, then there exists a curve $\alpha$, parameterized by arclength $s$, whose curvature and torsion functions are $\kappa$ and $\tau$. This theorem was obtained by Aoust in 1876. The first to discover the two curvatures of a curve in $\mathbb{E}^{3}$ was Monge in 1775 . He also obtained an analytic expression for the first curvature, but not for the torsion. This was done by Lancret in 1806. It was Cauchy, in his book on applications of the calculus to geometry of 1826 , who first made a study of space curves by systematically investigating its successive derivatives.

The initial study of surfaces in $\mathbb{E}^{3}$ began in a natural way. Since we have a theory of curves in the plane, we may hope to describe surfaces by investigating the curves in which the surface intersects various planes. The first results along this line, due to Euler, date from 1760 . Through a point $p$ on a surface $M \subset \mathbb{E}^{3}$ we construct the line $l$ which is perpendicular to the tangent plane at $M$ in $p$. For each unit vector $X$ in the tangent plane, we can consider the plane through $p$ which contains both $X$ and $l$. The intersection of this plane and $M$ is the image of a curve $\alpha_{X}$ with $\alpha_{X}(0)=p$; we will also suppose $\alpha_{X}$ parameterized by arclength, so that $\dot{\alpha}_{X}(0)=X$. We orient all these planes through $X$ and $l$ by choosing a vector $v_{p}$ perpendicular to the tangent plane at $M$ in $p$ and orienting the plane through $X$ and $l$ so that $X, v_{p}$ is positively oriented. Then $\alpha_{X}$ has a signed curvature at 0 , which will be denoted by $\kappa_{X}$. Euler discovered that if the $\kappa_{X}$ are not all equal, then there is precisely one direction, represented by a unit vector $X_{1}$, say, in which $\kappa_{X}$ has a minimum value $\kappa_{1}=\kappa_{X_{1}}$, and one in which it has a maximum value $\kappa_{2}=\kappa_{X_{2}}$. These two directions are perpendicular, and if $X$ makes an angle of $\theta$ with $X_{1}$, then

$$
\kappa_{X}=\kappa_{1} \cos ^{2} \theta+\kappa_{2} \sin ^{2} \theta .
$$

Despite the appealing simplicity of this construction, we never seem to really get our hands on the surface itself.

To do this we move forward 50 years in time where we find one of the most important works in the history of differential geometry, "Disquisitiones generales circa superficies 
curvas" of 1827 from Carl Friederich Gauss. At each point $p$ of $M$ there is a unique unit vector $\nu(p)$ such that $\nu(p)$ is perpendicular to the tangent plane at $M$ in $p$ and such that $\nu(p), v, w$ is positively oriented in $\mathbb{E}^{3}$ whenever $v$ and $w$ is positively oriented in the tangent plane at $M$ in $p$. Gauss defined the normal map $\nu$ - often called Gauss map - from the surface $M$ to $\mathbb{R}^{3}$, which actually goes to the unit sphere $S^{2} \subset \mathbb{R}^{3}$. The definition of the curvature $K(p)$ of $M$ at $p$ is given by

$$
K(p)=\lim _{A \rightarrow p} \frac{\operatorname{area} \nu(A)}{\operatorname{area} A}
$$

where the limit is taken as the region $A$ around $p$ becomes smaller and smaller. Gauss proved that this curvature $K(p)$ at any point $p \in M$ is the product of the extreme curvatures of the curves through $p$ cut out by normal planes,

$$
K(p)=\kappa_{1}(p) \kappa_{2}(p) .
$$

A remarkable theorem (in Latin Theorema Egregium) appears at the bottom of page 20 of Gauss' work :

Theorem. If a curved surface is developed upon any other surface whatever, the measure of curvature in each point remains unchanged.

What Gauss wants to say is that the curvature $K(p)$ in a point $p$ of a surface in $\mathbb{E}^{3}$ is an isometric invariant, not only an invariant under the group of Euclidean motions, but also invariant under the much larger group of maps, namely the isometries of surfaces. As a contrast, the mean curvature $H(p)$,

$$
H(p)=\frac{1}{2}\left(\kappa_{1}(p)+\kappa_{2}(p)\right),
$$

is invariant under the group of Euclidean motions, but is not invariant under the isometries of surfaces. So, besides the extrinsic geometry of surfaces in $\mathbb{E}^{3}$, which identifies two surfaces if and only if they differ only by a Euclidean motion in space, Gauss discovered that there is a basic geometric notion, his curvature, which is invariant under the group of isometries of surfaces, i.e. under those differentiable mappings between surfaces which preserve the distance function induced on the surface from the Euclidean metric of the ambient space. The study of the geometry of surfaces in $\mathbb{E}^{3}$ with these isometries as transformations is called the intrinsic geometry of the surfaces in $\mathbb{E}^{3}$.

On june 10, 1854 the faculty of Göttingen University heard the Inaugural lecture entitled "Über die Hypthesen, welche der Geometrie zu Grunde liegen" delivered by Georg Friederich Bernhard Riemann. The main contribution of Riemann to differential geometry was to actually carry out the program laid down in Gauss' Theorema Egregium, and such to develop a differential geometry for objects which do not have to be situated in a Euclidean space. Riemann proposes to consider a uniform method of assigning lengths of tangent vectors. He assumes that this length function $f$ is continuous on each tangent space and also positive homogeneous. The matrix of second order partial derivatives is positive semi-definite. Riemann then assumes, as the simplest possibility, that it is actually positive definite. An assignment to each tangent space of such a norm, or more precisely the inner product from which it comes, is what we now call a Riemannian 
metric on a manifold $M$. The corresponding intrinsic geometry is called Riemannian geometry. We can study Riemannian manifolds on their own (intrinsic geometry) or as submanifolds in some ambient Euclidean space (extrinsic geometry).

If the metric on a differentiable manifold is indefinite, we get a so-called semiRiemannian manifold, the corresponding geometry is the semi-Riemannian geometry. A semi-Riemannian manifold can also be seen as a submanifold in some semi-Euclidean space, so again we can work intrinsic or extrinsic.

The Minkowski space $\mathbb{E}_{1}^{n}$, i.e. the manifold $\mathbb{R}^{n}$ furnished with a metric tensor $g$ of index 1 , is a very important semi-Riemannian manifold. If $n=4$, it is the simplest example of a relativistic spacetime. The geometry of the Minkowski spacetime plays an important role in the study of special relativity. The serious difficulties in classical Newtonian physics were studied by Lorentz, Poincaré and Einstein. Einsteins mathematical essence was a novel way to change space and time coordinates; in 1908 Minkowski showed that these occur naturally if space $\mathbb{E}^{3}$ and time $\mathbb{E}$ are merged in a single spacetime $\mathbb{E}_{1}^{4}$. "Henceforth space by itself, and time by itself," he wrote, " are doomed to fade away into mere shadows, and only a kind of union of the two will preserve an independent reality."

In the first chapter of this dissertation we take a closer look at curves, which are one dimensional submanifolds, in the three- and four-dimensional Minkowski space.

We study the moving Frenet frames of curves in Minkowski space. The great differences between the study of Riemannian submanifolds and semi-Riemannian submanifolds appear immediatly in this chapter. For example the causal character of a curve plays an important role when we construct its adapted Frenet frame. Especially for null curves and curves with null normals there is a breakdown in the usual procedure for finding the Frenet equations, and it is simplest to use a moving frame with some null basis vectors. However, such a frame is not unique. The study of these special curves in the four-dimensional Minkowski space was done by W.B. Bonner [Bo1],[Bo2] and B. Rouxel [Ro1].

In section three of chapter 1 we classify all curves with constant curvatures in the three-dimensional Minkowski space. We'll make a distinction between spacelike, timelike and null curves. This last category is somewhat bizarre, in the sense that they have only one curvature. If for example this curvature is equal to zero, we get the well known null cubic.

The fourth and fifth section deal with curves of restricted type and curves of finite type in three dimensional Minkowski space. The notion of finite type submanifolds was introduced by B.y. Chen in [Ch1]. He defined a submanifold $M$ to be of finite type if the second component, $\mathrm{q}$, of the order $[\mathrm{p}, \mathrm{q}]$ of the submanifold $M$ is finite. This means that each component of its position vector field can be written as a finite sum of eigenfunctions of the Laplacian of $M$. Many results were already obtained. For example, Chen proved that a closed curve $\alpha$ in $\mathbb{R}^{n}$ is of finite type if and only if the Fourier series expansion of each coordinate function of $\alpha$ has only finite nonzero terms. If this curve $\alpha$ is a closed plane curve, then it is of finite type if and only if it is of type 1 and hence $\alpha$ is a circle. Chen, Deprez, Dillen, Verstraelen and Vrancken [CDDVV] studied space curves of finite type. In particular, they constructed examples of closed k-type curves in $\mathbb{E}^{3}$, for any positive integer $k$, and they characterized the circles as the only spherical 
curves of finite type in $\mathbb{E}^{3}$. We prove that all finite type curves in the Minkowski plane are of type 1, and hence a part of an orthogonal hyperbola or a part of a straight line. This result is analogous with the Riemannian case. Furthermore we classify all curves of Chen type 2 in $\mathbb{E}_{1}^{3}$.

Submanifolds of dimension two - normally called surfaces - in the three and $n$ dimensional Minkowski space are treated in chapter 2.

We first consider minimal surfaces, i.e. surfaces with mean curvature $H=0$. Kobayashi [Ko] and Van de Woestyne [VdW1] classified all minimal surfaces of revolution in $\mathbb{E}_{1}^{3}$, all minimal ruled surfaces in $\mathbb{E}_{1}^{3}$ and all minimal surfaces of translation in $\mathbb{E}_{1}^{3}$ with metric $g=d x^{2}+d y^{2}-d z^{2}$. By a surface of translation $M$ of $\mathbb{E}_{1}^{n}$, we mean a surface given by an immersion

$$
X: U \subset \mathbb{E}^{2} \rightarrow \mathbb{E}_{1}^{n}:(x, y) \rightarrow\left(x, y, f_{3}(x)+g_{3}(y), \ldots, f_{n}(x)+g_{n}(y)\right) .
$$

We classify all minimal surfaces of translation in the $n$-dimensional Minkowski space $\mathbb{E}_{1}^{n}$ with metric $g=-d x_{1}^{2}+\sum_{i=2}^{n} d x_{i}^{2}$.

In [CDVV1] is proved that a ruled surface of finite type in $\mathbb{E}^{n}$ is either a part of a cylinder over a curve of finite type or a helicoid in $\mathbb{E}^{3}$. In particular it follows that a ruled surface of finite type in $\mathbb{E}^{3}$ is a part of a plane, a circular cylinder or a helicoid. Dillen [Di] showed that a ruled submanifold of finite type in a Euclidean space is a cylinder on a curve of finite type or a generalized helicoid. In the third section of chapter 2 we give a classification of all ruled surfaces of finite type in $\mathbb{E}_{1}^{3}$. We obtain besides the minimal surfaces, and the expected circular and hyperbolic cylinders another class of ruled surfaces of finite type in $\mathbb{E}_{1}^{3}$, namely the isoparametric surfaces with null rules. Their mean curvature $H$ and Gauss curvature $K$ are constant and they are of null 2-type or of 1-type. An isoparametric surface with null rules is of 1-type if and only if it is a one-sheeted hyperboloid. A surface is of null 2-type if and only if its position vector field $X$ can be written as the sum of two eigenfunctions of the Laplacian with one of the corresponding eigenvalues equal to zero.

In the fourth section of chapter 2 we classify all ruled surfaces with constant mean curvature in $\mathbb{E}_{1}^{3}$. A surface of constant mean curvature in $\mathbb{E}_{1}^{3}$ is a surface in $\mathbb{E}_{1}^{3}$ for which the mean curvature vector field has constant length. The minimal ruled surfaces belong to this class of ruled surfaces with constant mean curvature and also the ruled surfaces of finite type are a part of this class. Moreover we prove that a surface in $\mathbb{E}_{1}^{3}$ has constant mean curvature if and only if it is of finite type in $\mathbb{E}_{1}^{3}$.

In the last chapter we explore the tensor product surfaces in $\mathbb{E}_{2}^{4}$, which are the product of a Lorentzian curve and a Euclidean curve. Let $\alpha: \mathbb{R} \rightarrow \mathbb{E}_{1}^{2}$ and $\beta: \mathbb{R} \rightarrow \mathbb{E}^{2}$ be respectively a Lorentzian planar curve and a Euclidean planar curve. Put $\alpha(t)=$ $\left(\alpha_{1}(t), \alpha_{2}(t)\right)$ and $\beta(s)=\left(\beta_{1}(s), \beta_{2}(s)\right)$. Then their tensor product is given by

$$
\alpha \otimes \beta: \mathbb{R}^{2} \rightarrow \mathbb{E}_{2}^{4}:(t, s) \rightarrow\left(\alpha_{1}(t) \beta_{1}(s), \alpha_{1}(t) \beta_{2}(s), \alpha_{2}(t) \beta_{1}(s), \alpha_{2}(t) \beta_{2}(s) .\right.
$$

The minimal tensor product surfaces are treated in section 2. A full classification of this class of surfaces was obtained. The tensor product $\alpha \otimes \beta$ is a minimal immersion 
if and only if either $\alpha$ or $\beta$ is a straight line through the origin, or both $\alpha$ and $\beta$ are orthogonal hyperbolae centered at the origin, or $\beta$ is a circle centered at the origin and $\alpha$ is either a circle or an orthogonal hyperbola centered at the origin.

In the next section the totally real and complex tensor product surfaces were studied. These two classes are special cases of slant surfaces. It was Chen [CH2] who introduced the notion of slant submanifolds. A slant submanifold $M$ is defined as a submanifold such that for any nonzero vector $X$ tangent at a point $p \in M$, the angle $\theta(X)$ between the tangent space in $p$ at $M$ and $J X$ is constant. $J$ is an almost complex structure on the ambient manifold. Complex and totally real immersions are slant immersions with $\theta=0$ and $\theta=\frac{\pi}{2}$, respectively. We prove that the tensor product $\alpha \otimes \beta$ is a totally real immersion with respect to the pseudo-Hermitian structure $J_{0}$,

$$
J_{0}(u, v, z, w)=(-v, u,-w, z),
$$

on $\mathbb{E}_{2}^{4}$ if and only if $\alpha$ is an orthogonal hyperbola centered at the origin, or $\beta$ is a straight line through the origin. We also prove that the tensor product $\alpha \otimes \beta$ is a complex immersion with respect to $J_{0}$ on $\mathbb{E}_{2}^{4}$ if and only if $\alpha$ is a straight line through the origin. The classification of proper slant - slant, but not totally real or complex product surfaces in $\mathbb{E}_{2}^{4}$ is still an open problem.

The last two sections deal with pseudo-minimal and pseudo-umbilical tensor product surfaces. A submanifold $M$ of a pseudo-Riemannian manifold $N$ is said to be pseudominimal if the length of its mean curvature vector vanishes identically. An isometric immersion is said to be pseudo-umbilical if the shape operator with respect to the mean curvature vector is proportional to the identity. For the first class we get a classification, namely that the tensor product $\alpha \otimes \beta$ is a non-minimal pseudo-minimal immersion if and only if $\alpha$ is an hyperbolic spiral in a Minkowski plane and $\beta$ is a logarithmic spiral in a Euclidean plane. For the pseudo-umbilical tensor product surfaces we have the following results. The tensor product of an hyperbola and a logarithmic spiral is pseudo-umbilical immersion in $\mathbb{E}_{2}^{4}$. Analogous is the product of a circle and an hyperbolic spiral a pseudoumbilical tensor product immersion in $\mathbb{E}_{2}^{4}$.

At the end we give some pictures of some of the surfaces which play an important role in this dissertation. 
CHAPTER I

\section{CURVES IN MINKOWSKI SPACE}

\section{§. INTRODUCTION}

Before studying curves in three- and four-dimensional Minkowski space, we first repeat some definitions related with semi-Riemannian geometry.

Let $V$ be a real vector space, then a bilinear form on $V$ is an $\mathbb{R}$-bilinear function $b: V \times V \rightarrow \mathbb{R}$.

Definition 1.1. A symmetric bilinear form $b$ on $\mathrm{V}$ is called

(1) positive definite provided $v \neq 0$ implies $b(v, v)>0$,

(2) negative definite provided $v \neq 0$ implies $b(v, v)<0$,

(3) nondegenerate provided $b(v, w)=0$ for all $w \in V$ implies $v=0$.

The index $\nu$ of a symmetric bilinear form $b$ on $V$ is the largest integer that is the dimension of a subspace $W \subset V$ on which $b \mid W$ is negative definite.

Definition 1.2. A metric tensor $g$ on a smooth manifold $M$ is a symmetric nondegenerate $(0,2)$ tensor field on $M$ of constant index.

In other words $g$ smoothly assigns to each point $p$ of $M$ a scalar product $g_{p}$ on the tangent space $T_{p}(M)$, and the index of $g_{p}$ is the same for all $p$.

Definition 1.3. A semi-Riemannian manifold is a smooth manifold $M$ furnished with a metric tensor $g$.

Thus strictly speaking a semi-Riemannian manifold is an ordered pair $(M, g)$ : two different metric tensors on the same manifold constitute different semi-Riemannian manifolds. Nevertheless we usually denote a semi-Riemannian manifold by the name of its smooth manifold $M, N, \ldots$, the metric $g$ we consider usually being clear from the context. We will work only with smooth manifolds, so we'll use the word "manifold" instead of "smooth manifold".

The common value $\nu$ of index $g_{p}$ on a semi-Riemannian manifold $M$ is called the index of $M: 0 \leq \nu \leq n=\operatorname{dim} M$.

If $\nu=0, M$ is a Riemannian manifold ; each $g_{p}$ is then a positive definite inner product on the tangent space $T_{p}(M)$ in $p$ at $M$.

If $\nu=1$ and $n \geq 2, M$ is a Lorentzian manifold. 
For any integer $\nu$ with $0 \leq \nu \leq n$, we have a metric tensor $g$ of index $\nu$ on $\mathbb{R}^{n}$,

$$
g(v, w)=-\sum_{i=1}^{\nu} v_{i} w_{i}+\sum_{j=\nu+1}^{n} v_{j} w_{j},
$$

with $v=\left(v_{1}, v_{2}, \ldots, v_{n}\right)$ and $w=\left(w_{1}, w_{2}, \ldots, w_{n}\right) \in \mathbb{R}^{n}$, or

$$
g=-\sum_{i=1}^{\nu} d x_{i}^{2}+\sum_{j=\nu+1}^{n} d x_{j}^{2}
$$

with $x_{1}, x_{2}, \ldots, x_{n}$ the natural coordinates on $\mathbb{R}_{\nu}^{n}$. The resulting semi-Euclidean space $\mathbb{E}_{\nu}^{n}$ reduces to the Euclidean space $\mathbb{E}^{n}$ if $\nu=0$. For $n \geq 2, \mathbb{E}_{1}^{n}$ is called the $n$-dimensional Minkowski space.

Definition 1.4. A vector $v$ tangent to a semi-Riemannian manifold $M$ is

(1) spacelike if $g(v, v)>0$ or $v=0$,

(2) null if $g(v, v)=0$ and $v \neq 0$,

(3) timelike if $g(v, v)<0$.

The set of all null vectors in the tangent space $T_{p}(M)$ is called the nullcone at a point $p \in M$. The category into which a given tangent vector falls is called its causal character.

The norm of a tangent vector $v$ is given by

$$
|v|=\sqrt{|g(v, v)|}
$$

and unit vectors, orthogonality and orthonormality are defined as in Euclidean spaces. Remark that not everything is the same as in Euclidean spaces. For example, the norm of a vector being null in a semi-Euclidean space doesn't mean that this vector is equal to 0 .

Let $M$ be a submanifold immersed by an immersion $i$ in a semi-Riemannian manifold $N$. If the pullback $i^{*}(g)$ is a metric tensor on $M$ it makes $M$ a semi-Riemannian submanifold of $N$.

Definition 1.5. A semi-Riemannian submanifold is called

(1) spacelike if $i^{*}(g)$ is positive definite,

(2) Lorentzian if $i^{*}(g)$ has index 1.

Let $x_{1}, x_{2}, \ldots, x_{n}$ be the natural coordinates on $\mathbb{E}_{\nu}^{n}$ and $\partial i=\frac{\partial}{\partial i}$. If $V$ and $W=$ $\sum_{i=1}^{n} W_{i} \partial_{i}$ are vector fields on $\mathbb{E}_{\nu}^{n}$, the vector field

$$
D_{V} W=\sum_{i=1}^{n} V\left(W_{i}\right) \partial_{i}
$$


is called the natural covariant derivative of $W$ with respect to $V$. The natural connection $D$ is the Levi-Civita connection of the semi-Euclidean space $\mathbb{E}_{\nu}^{n}$ for every $\nu=0,1, \ldots, n$.

The connection $D$ of $\mathbb{E}_{\nu}^{n}$ induces the Levi-Civita connection $\nabla$ of a semi-Riemannian submanifold $M$, which satisfies the following decomposition in a tangent and a normal component

$$
D_{X} Y=\nabla_{X} Y+h(X, Y)
$$

and

$$
D_{X} \xi=-A_{\xi} X+D_{X}^{\frac{1}{X}} \xi
$$

with $X, Y \in T_{p}(M), \xi$ a unit normal vector field on $M$. Further $h$ is called the second fundamental form of $M, A_{\xi}$ the shape operator of $M$ w.r.t. $\xi$ and $D^{\perp}$ is the normal connection of $M$. (1.1) is known as the the formula of Gauss, (1.2) as the formula of Weingarten .

We define a curve in the following way.

Definition 1.6. A curve in a manifold $M$ is a smooth mapping $\alpha: I \rightarrow M$, where $I$ is an open interval in the real line $\mathbb{R}$.

Let $x_{1}, \ldots, x_{n}$ be a local coordinate system in $M$ at a point $\alpha(s)$ of $\alpha$. The coordinate expression of the velocity vector of $\alpha$ is given by

$$
\dot{\alpha}(s)=\left.\sum_{i=1}^{n} \frac{d\left(x_{i} \circ \alpha\right)}{d u}(s) \partial_{i}\right|_{\alpha(s)}
$$

where $u$ is a coordinate system on $I$, namely the identity map of $I$. A curve $\alpha$ is said to be regular provided $\dot{\alpha}(s) \neq 0$ for all $s$.

If $\alpha: I \rightarrow M$ is a curve and $h: J \rightarrow I$ is a smooth function on an interval $J$, then $\beta=\alpha(h): J \rightarrow M$ is a curve called a reparameterisation of $\alpha$.

Then

$$
\dot{\beta}(t)=\left(\frac{d h}{d u}\right)(t) \dot{\alpha}(h(t))
$$

for all $t \in J$.

Definition 1.7. A curve $\alpha$ in a semi-Riemannian manifold $M$ is

(1) spacelike if all of its velocity vectors $\dot{\alpha}(s)$ are spacelike,

(2) null if all of its velocity vectors $\dot{\alpha}(s)$ are null,

(3) timelike if all of its velocity vectors $\dot{\alpha}(s)$ are timelike.

An arbitrary curve need not have one of these causal characters. 


\section{§. THE MOVING FRENET FRAME}

\subsection{The 3-dimensional Euclidean case.}

Consider a regular curve $\alpha(s)=\left(\alpha_{1}(s), \alpha_{2}(s), \alpha_{3}(s)\right)$ with arclength parameter $s$, that is $\forall s: g(\dot{\alpha}, \dot{\alpha})=1$, in the 3 -dimensional Euclidean space $\mathbb{E}^{3}$, with metric $g=$ $d x_{1}^{2}+d x_{2}^{2}+d x_{3}^{2}$. The Frenet frame along $\alpha$ is the orthonormal frame $\{T, N, B\}$ which is determined as follows. $T$ is the velocity or unit tangent vector field of $\alpha$. If $\ddot{\alpha}(s) \neq 0$, the acceleration $\ddot{\alpha}(s)$ of the curve $\alpha$ at the point $\alpha(s)$ is a vector which stands perpendicular to $T(s)$. The principal normal vector field $N$ is then the normalized acceleration field $\ddot{\alpha}$. The binormal vector field $B$ is determined by the vectorial product of $T$ and $N$. The Frenet formulas are given by

$$
\begin{aligned}
\dot{T} & =\kappa N \\
\dot{N} & =-\kappa T+\tau B \\
\dot{B} & =-\tau N
\end{aligned}
$$

or

$$
\left(\begin{array}{l}
\dot{T} \\
\dot{N} \\
\dot{B}
\end{array}\right)=\left(\begin{array}{ccc}
0 & \kappa & 0 \\
-\kappa & 0 & \tau \\
0 & -\tau & 0
\end{array}\right) \cdot\left(\begin{array}{l}
T \\
N \\
B
\end{array}\right)
$$

and determine all successive derivatives of $\alpha$. The functions $\kappa$ and $\tau$, which are respectively defined by the first and third formula, are called the first and second curvatures of $\alpha$, or the curvature and the torsion. The fundamental theorem of curves in $\mathbb{E}^{3}$ is,

Congruence theorem. If $\alpha, \beta: I \rightarrow \mathbb{E}^{3}$ are unit-speed curves such that

$$
\kappa_{\alpha}=\kappa_{\beta}>0
$$

and

$$
\tau_{\alpha}= \pm \tau_{\beta}
$$

then $\alpha$ and $\beta$ are congruent, i.e. are identical up to Euclidean motions.

The following properties characterize some special curves:

$\kappa=0$ if and only if $\alpha$ is a straight line,

$\tau=0$ if and only if $\alpha$ is a planar curve,

$\tau=0$ and $\kappa=$ constant $>0$ if and only if $\alpha$ is a circle,

$\tau=$ constant $\neq 0$ and $\kappa=$ constant $>0$ if and only if $\alpha$ is a circular helix.

What the Frenet theory for curves in Euclidean spaces does, is basically to assign a frame field on a curve, and then to express the derivatives of the basic vector fields with respect to this chosen Frenet frame. A frame field on a curve $\alpha: I \rightarrow M$ is defined as a set of mutually orthogonal unit vector fields on $\alpha$.In this way, the curvatures appear as coefficients in these Frenet formulas and completely determine the curves. This is essentially what is known as the method of moving frames for curves. 


\subsection{The 3-dimensional Minkowski case.}

We study the Frenet frames and formulas in the Minkowski space $\mathbb{E}_{1}^{3}, \mathbb{R}^{3}$ with metric $g=-d x_{1}^{2}+d x_{2}^{2}+d x_{3}^{2}$. A curve $\alpha(s)$ in $\mathbb{E}_{1}^{3}$ can have locally one of the following causal characters: $\alpha(s)$ is spacelike, null or timelike, which means that $g(\dot{\alpha}, \dot{\alpha})$ is bigger, equal or smaller then 0 on an interval $I \subset \mathbb{R}$. We consider these three cases seperately and construct their Frenet frame $\{T, N, B\}$.

Case 1. $\alpha$ is spacelike

We take the arclength parameter $s$ for $\alpha$ such that $g(\dot{\alpha}(s), \dot{\alpha}(s))=1 . T(s)$ is the velocity or unit tangent vector field of $\alpha(s)$. If $\ddot{\alpha}(s) \neq 0$, then $\ddot{\alpha}(s)$ is perpendicular to $T(s)$, so we take $N(s)=\lambda \ddot{\alpha}(s), \lambda \in \mathbb{R}$ and $\lambda>0$. Depending on the causal character of $\ddot{\alpha}(s)$ we have the following cases.

Case 1.1. $g(\ddot{\alpha}(s), \ddot{\alpha}(s))>0$

The principal normal vector field $N(s)$ is then the normalized vector field $\ddot{\alpha}(s)$. The binormal vector field $B(s)$ is the unique timelike unit vector field perpendicular to the spacelike plane $\{T(s), N(s)\}$ at every point $\alpha(s)$ of $\alpha$, such that $\{T, N, B\}$ has the same orientation as $\mathbb{E}_{1}^{3}$. The Frenet formulas are, in matrixnotation,

$$
\left(\begin{array}{c}
\dot{T} \\
\dot{N} \\
\dot{B}
\end{array}\right)=\left(\begin{array}{ccc}
0 & \kappa_{1} & 0 \\
-\kappa_{1} & 0 & \kappa_{2} \\
0 & \kappa_{2} & 0
\end{array}\right) \cdot\left(\begin{array}{c}
T \\
N \\
B
\end{array}\right) .
$$

Case 1.2. $g(\ddot{\alpha}(s), \ddot{\alpha}(s))<0$

The principal normal vector field $N(s)$ is then the normalized timelike vector field $\ddot{\alpha}(s)$. The binormal vector field $B(s)$ is the unique spacelike unit vector field perpendicular to the timelike plane $\{T(s), N(s)\}$ at every point $\alpha(s)$ of $\alpha$, such that $\{T, N, B\}$ has the same orientation as $\mathbb{E}_{1}^{3}$. The Frenet formulas are, in matrixnotation,

$$
\left(\begin{array}{c}
\dot{T} \\
\dot{N} \\
\dot{B}
\end{array}\right)=\left(\begin{array}{ccc}
0 & \kappa_{1} & 0 \\
\kappa_{1} & 0 & \kappa_{2} \\
0 & \kappa_{2} & 0
\end{array}\right) \cdot\left(\begin{array}{c}
T \\
N \\
B
\end{array}\right) .
$$

Case 1.3. $g(\ddot{\alpha}(s), \ddot{\alpha}(s))=0$

To rule out straight lines and points of inflexion on $\alpha$, we shall suppose that $\ddot{\alpha}(s) \neq 0$ - The principal normal vector field $N(s)$ is then the vector field $\ddot{\alpha}(s)$. The binormal vector field $B(s)$ is the unique null vector field perpendicular to $T(s)$ at every point $\alpha(s)$ of $\alpha$, such that $g(N, B)=1$. The Frenet formulas are, in matrixnotation,

$$
\left(\begin{array}{c}
\dot{T} \\
\dot{N} \\
\dot{B}
\end{array}\right)=\left(\begin{array}{ccc}
0 & \kappa_{1} & 0 \\
0 & \kappa_{2} & 0 \\
-\kappa_{1} & 0 & -\kappa_{2}
\end{array}\right) \cdot\left(\begin{array}{c}
T \\
N \\
B
\end{array}\right)
$$

where the " curvature " $\kappa_{1}$ can only take two values; 0 , when $\alpha$ is a straight line, or 1 in all other cases. If $\alpha(s)$ is a straight line, then $\ddot{\alpha}(s)=0=\dot{T}(s)$ which means that $\kappa_{1}=0$. If $\alpha(s)$ is not a straight line, then there exist an interval $I$ on which $\ddot{\alpha}(s) \neq 0$. 
$N(s)$ is defined as $N(s)=\ddot{\alpha}(s)=\dot{T}(s)$, thus $\kappa_{1}=1 .\{T, N, B\}$ is a pseudo-orthonormal basis in $\mathbb{E}_{1}^{3}$, which means that

$$
\begin{aligned}
& \dot{N}=a_{1} T+a_{2} N+a_{3} B \\
& \dot{B}=b_{1} T+b_{2} N+b_{3} B .
\end{aligned}
$$

From

$$
g(N, N)=g(N, T)=g(B, B)=0
$$

we get respectively that $a_{3}=a_{1}=b_{2}=0$. Considering that

$$
g(N, B)=1 \text { and } g(B, T)=0,
$$

we get by differentiation that

$$
g(\dot{N}, B)+g(N, \dot{B})=0 \text { and } g(\dot{B}, T)+g(B, \dot{T})=0,
$$

which means that

$$
a_{2}=-b_{3} \text { and } b_{1}=-\kappa_{1}=-1 .
$$

Concluding, we see that in this case there is only one curvature $a_{2}=\kappa_{2}$.

Case 2. $\alpha$ is timelike

We take the arclength parameter $s$ for $\alpha$ such that $g(\dot{\alpha}(s), \dot{\alpha}(s))=-1$. $T(s)$ is the unit timelike tangent vector field of $\alpha . \ddot{\alpha}(s)$ is perpendicular to $T(s)$, and thus spacelike, so we define the principal normal field $N(s)$ as the normalized vector field $\ddot{\alpha}(s)$. The binormal vector field $B(s)$ is the unique spacelike unit vector field perpendicular to the timelike plane $\{T(s), N(s)\}$ at every point $\alpha(s)$ of $\alpha$, such that $\{T, N, B\}$ has the same orientation as $\mathbb{E}_{1}^{3}$. The Frenet formulas are, in matrixnotation,

$$
\left(\begin{array}{c}
\dot{T} \\
\dot{N} \\
\dot{B}
\end{array}\right)=\left(\begin{array}{ccc}
0 & \kappa_{1} & 0 \\
\kappa_{1} & 0 & \kappa_{2} \\
0 & -\kappa_{2} & 0
\end{array}\right) \cdot\left(\begin{array}{l}
T \\
N \\
B
\end{array}\right) .
$$

Case 3. $\alpha$ is a null-curve

Let $T$ be the null vector field $\dot{\alpha}$, then $\ddot{\alpha}$ is a spacelike vector field perpendicular to $T$, except when $\ddot{\alpha}=0$. If $\alpha$ is not a straight null line, take then as parameter the pseudo-arclenght $s$, i.e. $g(\ddot{\alpha}(s), \ddot{\alpha}(s))=1, \quad \forall s$, and define $N$ as the unit vector field corresponding to $\ddot{\alpha}$. The binormal vector field $B$ is the unique null vector field perpendicular to $N(s)$ at every point $\alpha(s)$ of $\alpha$, such that $g(T, B)=1$. The Frenet formulas are, in matrixnotation,

$$
\left(\begin{array}{c}
\dot{T} \\
\dot{N} \\
\dot{B}
\end{array}\right)=\left(\begin{array}{ccc}
0 & \kappa_{1} & 0 \\
\kappa_{2} & 0 & -\kappa_{1} \\
0 & -\kappa_{2} & 0
\end{array}\right) \cdot\left(\begin{array}{l}
T \\
N \\
B
\end{array}\right),
$$

where the "curvature" $\kappa_{1}$ can only take two values; 0 , when $\alpha$ is a straight null line, or 1 in all other cases. If $\alpha(s)$ is a straight null line, then $\ddot{\alpha}(s)=0=\dot{T}(s)$ which means that 
$\kappa_{1}=0$. If $\alpha(s)$ is not a straight line, then there exist an interval $I$ on which $\ddot{\alpha}(s) \neq 0$. $N(s)$ is defined as $N(s)=\ddot{\alpha}(s)=\dot{T}(s)$, thus $\kappa_{1}=1 .\{T, N, B\}$ is a pseudo-orthonormal basis in $\mathbb{E}_{1}^{3}$, which means that

$$
\begin{aligned}
& \dot{N}=a_{1} T+a_{2} N+a_{3} B \\
& \dot{B}=b_{1} T+b_{2} N+b_{3} B .
\end{aligned}
$$

From

$$
g(N, N)=g(T, B)=1 \text { and } g(B, B)=0
$$

we get respectively that $a_{2}=b_{3}=b_{1}=0$. Considering that

$$
g(T, N)=g(N, B)=0,
$$

we get by differentiation that

$$
g(\dot{T}, N)+g(T, \dot{N})=0 \text { and } g(\dot{N}, B)+g(N, \dot{B})=0
$$

which means that

$$
a_{3}=-\kappa_{1}=-1 \text { and } a_{1}=-b_{2} .
$$

Also in this case we see that there is only one curvature $a_{1}=\kappa_{2}$. 


\subsection{The 4-dimensional Euclidean case.}

Consider a regular curve $\alpha(s)=\left(\alpha_{1}(s), \alpha_{2}(s), \alpha_{3}(s), \alpha_{4}(s)\right)$ with arclength parameter $s, \forall s: g(\dot{\alpha}, \dot{\alpha})=1$, in the 4 -dimensional Euclidean space $\mathbb{E}^{4}$, with metric $g=d x_{1}^{2}+d x_{2}^{2}+d x_{3}^{2}+d x_{4}^{2}$. The Frenet frame along $\alpha$ is the orthonormal frame $\left\{T, N, B_{1}, B_{2}\right\}$ which is determined as follows. $T$ is the velocity or unit tangent vector field of $\alpha$. If $\ddot{\alpha}(s) \neq 0$, then the acceleration $\ddot{\alpha}(s)$ of the curve $\alpha$ at the point $\alpha(s)$ is a vector which stands perpendicular to $T(s)$. The principal normal vector field $N$ is the normalized acceleration field $\ddot{\alpha}$. The unit vector field $B_{1}$ is determined such that $\dot{N}$ can be decomposed into two components, a tangent one in the direction of $T$ and a normal one in the direction of $B_{1} . B_{2}$ is the unique unit vector field perpendicular to the three-dimensional subspace $\left\{T, N, B_{1}\right\}$, such that the orientation of the frame $\left\{T, N, B_{1}, B_{2}\right\}$ is the same as the orientation of $\mathbb{E}^{4}$. The Frenet formulas are given by

$$
\begin{aligned}
& \dot{T}=\kappa_{1} N \\
& \dot{N}=-\kappa_{1} T+\kappa_{2} B_{1} \\
& \dot{B}_{1}=-\kappa_{2} N+\kappa_{3} B_{2} \\
& \dot{B}_{2}=-\kappa_{3} B_{1}
\end{aligned}
$$

or

$$
\left(\begin{array}{c}
\dot{T} \\
\dot{N} \\
\dot{B}_{1} \\
\dot{B}_{2}
\end{array}\right)=\left(\begin{array}{cccc}
0 & \kappa_{1} & 0 & 0 \\
-\kappa_{1} & 0 & \kappa_{2} & 0 \\
0 & -\kappa_{2} & 0 & \kappa_{3} \\
0 & 0 & -\kappa_{3} & 0
\end{array}\right) \cdot\left(\begin{array}{c}
T \\
N \\
B_{1} \\
B_{2}
\end{array}\right)
$$

and determine all succesive derivatives of $\alpha$. The functions $\kappa_{1}, \kappa_{2}$ and $\kappa_{3}$ are called the first, second and third curvatures of $\alpha$. The fundamental theorem of curves in $\mathbb{E}^{4}$ states that two curves for which the absolute values of the curvatures are the same, are congruent, i.e. are identical up to Euclidean motions.

The following properties characterize some special curves:

$\kappa_{1}=0$ if and only if $\alpha$ is a straight line,

$\kappa_{2}=0$ if and only if $\alpha$ is a planar curve,

$\kappa_{3}=0$ if and only if $\alpha$ is lying in a three dimensional subspace of $\mathbb{E}^{4}$,

$\kappa_{2}=0$ and $\kappa_{1}=$ constant $>0$ if and only if $\alpha$ is a circle,

$\kappa_{3}=0$ and $\kappa_{2}=c_{2}, \quad \kappa_{1}=c_{1}, \quad c_{1}, c_{2} \in \mathbb{R}_{0}$ if and only if $\alpha$ is a circular helix,

$\kappa_{3}=c_{3}, \quad \kappa_{2}=c_{2}, \quad \kappa_{1}=c_{1}, \quad c_{1}, c_{2}, c_{3} \in \mathbb{R}_{0}$ if and only if

$$
\alpha(s)=\frac{1}{\lambda_{1}} \sin \left(\lambda_{1} s\right) \cdot V_{1}-\frac{1}{\lambda_{1}} \cos \left(\lambda_{1} s\right) \cdot V_{2}+\frac{1}{\lambda_{2}} \sin \left(\lambda_{2} s\right) \cdot V_{3}-\frac{1}{\lambda_{2}} \cos \left(\lambda_{2} s\right) \cdot V_{4}
$$

with $\lambda_{1}^{2}=\frac{K-\sqrt{K^{2}-4 c_{1}^{2} c_{3}^{2}}}{2}, \quad \lambda_{2}^{2}=\frac{K+\sqrt{K^{2}-4 c_{1}^{2} c_{3}^{2}}}{2}$ and $K=c_{1}^{2}+c_{2}^{2}+c_{3}^{2}, \quad V_{i}$ are orthogonal, constant vectors satisfying the following conditions $g\left(V_{1}, V_{1}\right)=g\left(V_{2}, V_{2}\right)$ and $g\left(V_{3}, V_{3}\right)=g\left(V_{4}, V_{4}\right)$. This curve $\alpha$ lies on a sphere with radius $\frac{1}{\left|c_{3}\right|}$. 


\subsection{The 4-dimensional Minkowski case.}

We study the Frenet frames and formulas in the Minkowski space $\mathbb{E}_{1}^{4}, \mathbb{R}^{4}$ with metric $g=-d x_{1}^{2}+d x_{2}^{2}+d x_{3}^{2}+d x_{4}^{2}$. A curve $\alpha(s)$ in $\mathbb{E}_{1}^{4}$ can have locally one of the following causal characters: $\alpha(s)$ is spacelike, null or timelike, which means that $g(\dot{\alpha}, \dot{\alpha})$ is bigger, equal or smaller then 0 on an interval $I$. We will look at these three cases seperately and construct their Frenet frame $\left\{T, N, B_{1}, B_{2}\right\}$.

Case 1. $\alpha$ is spacelike

We take the arclength parameter $s$ for $\alpha$ such that $g(\dot{\alpha}(s), \dot{\alpha}(s))=1$. T is the unit tangent vector field of $\alpha$. If $\ddot{\alpha} \neq 0$, then $\ddot{\alpha}$ is perpendicular to $T$, so we take $N$ in the direction of $\ddot{\alpha}$. Depending on the causal character of $\ddot{\alpha}$ we have the following cases.

Case 1.1. $g(\ddot{\alpha}(s), \ddot{\alpha}(s))>0$

The principal normal vector field $N$ is then the normalized vector field corresponding to $\ddot{\alpha}(s)$. The vector field $B_{1}$ is in the direction of the normal component $C^{\perp}$ of $\dot{N}$ with respect to the plane $\{T, N\}$ and can have all the causal characters.

Case $1.1 .1 \mathrm{~g}\left(\mathrm{C}^{\perp}, \mathrm{C}^{\perp}\right)>0$

Then $B_{1}$ is the normalized vector field $C^{\perp}$ and $B_{2}$ is the unique timelike unit vector field perpendicular to the 3 -dimensional subspace $\left\{T, N, B_{1}\right\}$, such that the orientation of $\left\{T, N, B_{1}, B_{2}\right\}$ is the same as that of $\mathbb{E}_{1}^{4}$. The Frenet formulas then become

$$
\left(\begin{array}{c}
\dot{T} \\
\dot{N} \\
\dot{B}_{1} \\
\dot{B}_{2}
\end{array}\right)=\left(\begin{array}{cccc}
0 & \kappa_{1} & 0 & 0 \\
-\kappa_{1} & 0 & \kappa_{2} & 0 \\
0 & -\kappa_{2} & 0 & \kappa_{3} \\
0 & 0 & \kappa_{3} & 0
\end{array}\right) \cdot\left(\begin{array}{c}
T \\
N \\
B_{1} \\
B_{2}
\end{array}\right) \text {. }
$$

Case 1.1.2 $g\left(C^{\perp}, C^{\perp}\right)<0$

Then $B_{1}$ is the timelike normalized vector field $C^{\perp}$ and $B_{2}$ is the unique spacelike unit vector field perpendicular to the 3 -dimensional subspace $\left\{T, N, B_{1}\right\}$, such that the orientation of $\left\{T, N, B_{1}, B_{2}\right\}$ is the same as that of $\mathbb{E}_{1}^{4}$. The Frenet formulas then become

$$
\left(\begin{array}{c}
\dot{T} \\
\dot{N} \\
\dot{B}_{1} \\
\dot{B}_{2}
\end{array}\right)=\left(\begin{array}{cccc}
0 & \kappa_{1} & 0 & 0 \\
-\kappa_{1} & 0 & \kappa_{2} & 0 \\
0 & \kappa_{2} & 0 & \kappa_{3} \\
0 & 0 & -\kappa_{3} & 0
\end{array}\right) \cdot\left(\begin{array}{c}
T \\
N \\
B_{1} \\
B_{2}
\end{array}\right) \text {. }
$$

Case $1.1 .3 \mathrm{~g}\left(\mathrm{C}^{\perp}, \mathrm{C}^{\perp}\right)=0$

Then $B_{1}$ is the vector field $C^{\perp}$ and $B_{2}$ is the unique null vector field perpendicular to the plane $\{T, N\}$, such that $g\left(B_{1}, B_{2}\right)=1$. The Frenet formulas then become

$$
\left(\begin{array}{c}
\dot{T} \\
\dot{N} \\
\dot{B}_{1} \\
\dot{B}_{2}
\end{array}\right)=\left(\begin{array}{cccc}
0 & \kappa_{1} & 0 & 0 \\
-\kappa_{1} & 0 & \kappa_{2} & 0 \\
0 & 0 & \kappa_{3} & 0 \\
0 & -\kappa_{2} & 0 & -\kappa_{3}
\end{array}\right) \cdot\left(\begin{array}{c}
T \\
N \\
B_{1} \\
B_{2}
\end{array}\right) \text {. }
$$


Such a curve $\alpha$ is called a partially null curve and lies in a three dimensional subspace, as we see from the Frenet formulas. By making a null rotation, i.e. a map from one null tetrad to another null tetrad, that keeps $T$ and $N$ fixed, we can make $\kappa_{3}=0$. Such that $B_{1}$ is a constant null vector. Working out this case, we make the following null rotation

$$
\left(\begin{array}{l}
\bar{T} \\
\bar{N} \\
\bar{B}_{1} \\
\bar{B}_{2}
\end{array}\right)=\left(\begin{array}{llll}
1 & 0 & 0 & 0 \\
0 & 1 & 0 & 0 \\
0 & 0 & \frac{1}{a} & 0 \\
0 & 0 & 0 & a
\end{array}\right) \cdot\left(\begin{array}{c}
T \\
N \\
B_{1} \\
B_{2}
\end{array}\right) .
$$

The Frenet formulas then become

$$
\left(\begin{array}{c}
\dot{\bar{T}} \\
\dot{\bar{N}} \\
\dot{\bar{B}}_{1} \\
\dot{\bar{B}}_{2}
\end{array}\right)=\left(\begin{array}{cccc}
0 & \bar{\kappa}_{1} & 0 & 0 \\
-\bar{\kappa}_{1} & 0 & \bar{\kappa}_{2} & 0 \\
0 & 0 & \bar{\kappa}_{3} & 0 \\
0 & -\bar{\kappa}_{2} & 0 & -\bar{\kappa}_{3}
\end{array}\right) \cdot\left(\begin{array}{c}
\bar{T} \\
\bar{N} \\
\bar{B}_{1} \\
\bar{B}_{2}
\end{array}\right)
$$

with

$$
\bar{\kappa}_{1}=\kappa_{1}, \bar{\kappa}_{2}=a \kappa_{2} \text { and } \bar{\kappa}_{3}=\frac{-\dot{a}}{a}+\kappa_{3} .
$$

Thus we may choose $a(s)$ so that $\bar{\kappa}_{3}=0$. This means that

$$
\dot{\bar{B}}_{1}=0
$$

or in other words $\bar{B}_{1}$ is a constant null vector. There are only two curvatures in this case, the second curvature is determined only up to a constant factor. For more details we refer to [Bo2]. A special partially null curve is the partially null helix, i.e. a curve that satisfies the above Frenet formulas with $\kappa_{1}$ and $\kappa_{2}$ non-zero constants. This curve may be expressed as

$$
\alpha(s)=\left(c s, \frac{1}{\kappa_{1}} \cos \left(\kappa_{1} s\right), \frac{1}{\kappa_{1}} \sin \left(\kappa_{1} s\right), c s\right),
$$

with $c$ a non-zero constant and may be regarded as a circular helix with a null axis.

Case 1.2. $g(\ddot{\alpha}(s), \ddot{\alpha}(s))<0$

The principal normal vector field $N$ is then the normalized timelike vector field corresponding to $\ddot{\alpha}(s)$. The unit vector field $B_{1}$ is in the direction of the normal component of $\dot{N}$ and must be spacelike. $B_{2}$ is the unique unit spacelike vector field perpendicular to the subspace $\left\{T, N, B_{1}\right\}$ and such that the orientation of $\left\{T, N, B_{1}, B_{2}\right\}$ is equal to that of $\mathbb{E}_{1}^{4}$. The Frenet formulas are

$$
\left(\begin{array}{c}
\dot{T} \\
\dot{N} \\
\dot{B}_{1} \\
\dot{B}_{2}
\end{array}\right)=\left(\begin{array}{cccc}
0 & \kappa_{1} & 0 & 0 \\
\kappa_{1} & 0 & \kappa_{2} & 0 \\
0 & \kappa_{2} & 0 & \kappa_{3} \\
0 & 0 & -\kappa_{3} & 0
\end{array}\right) \cdot\left(\begin{array}{c}
T \\
N \\
B_{1} \\
B_{2}
\end{array}\right) .
$$

Case 1.3. $g(\ddot{\alpha}(s), \ddot{\alpha}(s))=0$

Such a curve $\alpha$ is known as a pseudo null curve. $N$ is the vector field $\ddot{\alpha}$, if $\ddot{\alpha}(s) \neq 0$. 
$\dddot{\alpha}$ can be a spacelike vector field or a null vector field. If $\dddot{\alpha}$ is spacelike, let then $B_{1}$ be the normalized vector field $\dddot{\alpha} . B_{2}$ is the unique null vector field perpendicular to the subspace $\left\{T, B_{1}\right\}$, such that $g\left(N, B_{2}\right)=1$. The Frenet system - known as the Standard Tetrad [Bo2] - then becomes

$$
\left(\begin{array}{c}
\dot{T} \\
\dot{N} \\
\dot{B}_{1} \\
\dot{B}_{2}
\end{array}\right)=\left(\begin{array}{cccc}
0 & \kappa_{1} & 0 & 0 \\
0 & 0 & \kappa_{2} & 0 \\
0 & \kappa_{3} & 0 & -\kappa_{2} \\
-\kappa_{1} & 0 & -\kappa_{3} & 0
\end{array}\right) \cdot\left(\begin{array}{c}
T \\
N \\
B_{1} \\
B_{2}
\end{array}\right) .
$$

where the "curvature" $\kappa_{1}$ can only take two values; 0 , when $\alpha$ is a straight null line, or 1 in all other cases.

If $\dddot{\alpha}$ is a null vector field, then $\alpha$ is a two dimensional pseudo null curve, lying in a degenerate plane, with parameterisation

$$
\alpha(s)=(f(s), s, 0, f(s))
$$

where $f$ is an arbitrary function of $s$. From any pseudo null curve $\alpha$ we can obtain the associated null curve $\tilde{\alpha}$ see [Ro1]

$$
\tilde{\alpha}(s)=\alpha(s)-s \cdot T(s)
$$

and in this case the associated null curve is a straight line.

Case 2. $\alpha$ is timelike

We take the arclenght parameter $s$ for $\alpha$ such that $g(\dot{\alpha}(s), \dot{\alpha}(s))=-1$. $T$ is the timelike unit vector field $\dot{\alpha}$. $\ddot{\alpha}$ is perpendicular to $T$, so $N$ is the spacelike normalized vector field $\ddot{\alpha} . B_{1}$ is a spacelike unit vector field in the direction of the normal component of $\dot{N}$ with respect to the plane $\{T, N\} . B_{2}$ is the unique spacelike unit vector field perpendicular to $\left\{T, N, B_{1}\right\}$ and such that the orientation of $\left\{T, N, B_{1}, B_{2}\right\}$ corresponds with that of $\mathbb{E}_{1}^{4}$. The Frenet formulas are

$$
\left(\begin{array}{c}
\dot{T} \\
\dot{N} \\
\dot{B}_{1} \\
\dot{B}_{2}
\end{array}\right)=\left(\begin{array}{cccc}
0 & \kappa_{1} & 0 & 0 \\
\kappa_{1} & 0 & \kappa_{2} & 0 \\
0 & -\kappa_{2} & 0 & \kappa_{3} \\
0 & 0 & -\kappa_{3} & 0
\end{array}\right) \cdot\left(\begin{array}{c}
T \\
N \\
B_{1} \\
B_{2}
\end{array}\right) .
$$

Case 3. $\alpha$ is a null curve

Let $T$ be the null vector field $\dot{\alpha}$. If we exclude straight lines and use the pseudo-arc $s$ for $\alpha$, then $g(\ddot{\alpha}(s), \ddot{\alpha}(s))=1$, so take $N=\ddot{\alpha}$. Let $B_{1}$ be the normal component of $\dddot{\alpha}$ with respect to the plane $\{T, N\}$. From $g(\dot{\alpha}, \dddot{\alpha})=-1$ and $g(\ddot{\alpha}, \dddot{\alpha})=0$, we get that $g\left(B_{1}, B_{1}\right)$ must be equal to 0 and $B_{1}$ is completely determined by $g\left(T, B_{1}\right)=1$. $B_{2}$ is the unique spacelike unit vector field perpendicular to the 3-dimensional subspace $\left\{T, N, B_{1}\right\}$ and such that the orientation of $\left\{T, N, B_{1}, B_{2}\right\}$ is the same as that of $\mathbb{E}_{1}^{4}$. The Frenet formulas are

$$
\left(\begin{array}{c}
\dot{T} \\
\dot{N} \\
\dot{B}_{1} \\
\dot{B}_{2}
\end{array}\right)=\left(\begin{array}{cccc}
0 & \kappa_{1} & 0 & 0 \\
\kappa_{2} & 0 & -\kappa_{1} & 0 \\
0 & -\kappa_{2} & 0 & \kappa_{3} \\
-\kappa_{3} & 0 & 0 & 0
\end{array}\right) \cdot\left(\begin{array}{c}
T \\
N \\
B_{1} \\
B_{2}
\end{array}\right) .
$$


where the "curvature" $\kappa_{1}$ can only take two values; 0 , when $\alpha$ is a straight null line, or 1 in all other cases.

This tetrad $\left\{T, N, B_{1}, B_{2}\right\}$ is known as the Cartan tetrad for null curves, for other tetrads as the canonical tetrad or the screw tetrad see [Bol].

A null curve has only two essential curvatures $\kappa_{2}$ and $\kappa_{3} . \kappa_{3}$ plays a role similar to the third curvature of a curve in $\mathbb{E}^{4}$, this means that a null curve $\alpha$ lies in a three dimensional subspace of $\mathbb{E}_{1}^{4}$ if and only if $\kappa_{3}=0$. The geometrical meaning of $\kappa_{2}$ is less obvious. There exist null helices with $\kappa_{2}=0$, and there is a single curve, the null cubic, with $\kappa_{2}=\kappa_{3}=0$. For this reason, we study null curves with constant curvatures.

Case 1. $\kappa_{2}=\kappa_{3}=0$

From the Frenet equations we have that

$$
\dddot{\alpha}=0 \text {. }
$$

Thus

$$
\alpha(s)=A s^{3}+B s^{2}+C s+D, \quad A, B, C, D \in \mathbb{R}^{4},
$$

and the derivatives are

$$
\begin{gathered}
\dot{\alpha}(s)=3 A s^{2}+2 B s+C, \\
\ddot{\alpha}(s)=6 A s+2 B, \\
\dddot{\alpha}(s)=6 A .
\end{gathered}
$$

Because $g\left(B_{1}, B_{1}\right)=0=g(\dddot{\alpha}, \dddot{\alpha})$, we get that $A$ is a constant null vector. Expressing that $s$ is the pseudo-arc parameter, i.e. $g(\ddot{\alpha}, \ddot{\alpha})=1$, and that $\alpha$ is a null curve, i.e. $g(\dot{\alpha}, \dot{\alpha})=0$, we get the following conditions on the vectors $A, B, C, D$,

$$
g(A, A)=g(C, C)=g(A, B)=g(B, C)=0, \quad g(A, C)=\frac{-1}{6}, \quad g(B, B)=\frac{1}{4} .
$$

For example

$$
\alpha(s)=\left(s^{3}+\frac{s}{12}, s^{3}-\frac{s}{12}, \frac{s^{2}}{2}, 0\right)
$$

which is a parameterisation of the null cubic.

Case 2. $\kappa_{2}, \kappa_{3}$ constants and not both zero

Case 2.1. $\kappa_{3} \neq 0$

We have to solve

$$
\frac{d^{4} T}{d s^{4}}-2 \kappa_{2} \frac{d^{2} T}{d s^{2}}-\kappa_{3}^{2} T=0
$$

After integration, we get

$$
\alpha(s)=\frac{1}{\lambda_{1}}\left[\sinh \left(\lambda_{1} s\right) V+\cosh \left(\lambda_{1} s\right) W\right]+\frac{1}{\lambda_{2}}\left[\sin \left(\lambda_{2} s\right) Z-\cos \left(\lambda_{2} s\right) Y\right]
$$

with

$$
g(Z, Z)=g(W, W)=g(Y, Y)=-g(V, V)=\frac{1}{\lambda_{1}^{2}+\lambda_{2}^{2}},
$$




$$
\lambda_{1}=\sqrt{\sqrt{\kappa_{2}^{2}+\kappa_{3}^{2}}+\kappa_{2}}, \quad \lambda_{2}=\sqrt{\sqrt{\kappa_{2}^{2}+\kappa_{3}^{2}}-\kappa_{2}} .
$$

This is a parameterisation of a null helix, lying on a circular cylinder.

No very distinctive role is played by $\kappa_{2}$, it can be put zero without affecting the general nature of the helix.

Case 2.2. $\kappa_{3}=0$

If $\kappa_{3}=0$, the curves are lying in a three dimensional subspace. From the Frenet equations follows that we have to solve the differential equation

$$
\frac{d^{3} T}{d s^{3}}=2 \kappa_{2} \frac{d T}{d s}
$$

We distinguish two cases :

i) $\kappa_{2}>0$, then

$$
\alpha(s)=\frac{1}{2 \kappa_{2}}\left[\sinh \left(\sqrt{2 \kappa_{2}} s\right), \cosh \left(\sqrt{2 \kappa_{2}} s\right), \sqrt{2 \kappa_{2}} s, 0\right] .
$$

ii) $\kappa_{2}<0$, then

$$
\alpha(s)=\frac{1}{-2 \kappa_{2}}\left[0, \sqrt{-2 \kappa_{2}} s, \sin \left(\sqrt{-2 \kappa_{2}} s\right), \cos \left(\sqrt{-2 \kappa_{2}} s\right)\right] .
$$




\section{§3. CURVES WITH CONSTANT CURVATURE IN $\mathbb{E}_{1}^{3}$}

In the 3-dimensional Euclidean space $\mathbb{E}^{3}$, we know that every curve $\alpha$ has two curvatures, the first curvature $\kappa_{1}$, also called the curvature $\kappa$, and the second curvature $\kappa_{2}$, sometimes called the torsion $\tau$. Curves with constant curvatures - also called W-curves - are characterized by the following properties :

$\kappa=0$ if and only if $\alpha$ is a straight line,

$\tau=0$ if and only if $\alpha$ is a planar curve,

$\tau=0$ and $\kappa=$ constant $>0$ if and only if $\alpha$ is a circle,

$\tau=$ constant $\neq 0$ and $\kappa=$ constant $>0$ if and only if $\alpha$ is a circular helix.

In the next sections we study W-curves, i.e. curves with constant curvatures, in Minkowski space.

\subsection{Spacelike curves.}

We will work with the Frenet equations from the previous section, so we shall distinguish three cases depending on the sign of $g(\ddot{\alpha}, \ddot{\alpha})$. Before doing this, we give some general results for spacelike curves in $\mathbb{E}_{1}^{3}$.

Theorem 3.1. A spacelike curve $\alpha$ in $\mathbb{E}_{1}^{3}$ has first curvature $\kappa_{1}$ identically zero if and only if $\alpha$ is a part of a straight line.

Proof.

From the Frenet equations follows that $\kappa_{1}$ equal to zero, is equivalent with $\dot{T}=\ddot{\alpha}=0$, which means that $\alpha$ is a part of a straight line.

Thus first curvature measures deviation from straightness.

Theorem 3.2. A spacelike curve $\alpha$ in $\mathbb{E}_{1}^{3}$, with $g(\ddot{\alpha}, \ddot{\alpha}) \neq 0$, has second curvature $\kappa_{2}$ identically zero if and only if $\alpha$ is a planar curve.

Proof.

If $\kappa_{2}=0$, we get from the Frenet equations that

$$
\begin{gathered}
\dot{T}=\kappa_{1} N \\
\dot{N}= \pm \kappa_{1} T .
\end{gathered}
$$

Rewriting this equations in terms of the derivatives of the curve $\alpha$ gives us the following equation,

$$
\dddot{\alpha}=\frac{\dot{\kappa}_{1}}{\kappa_{1}} \ddot{\alpha} \pm \kappa_{1}^{2} \dot{\alpha} .
$$

Using the MacLaurin expansion for $\alpha$,

$$
\alpha(s)=\alpha(0)+\dot{\alpha}(0) s+\ddot{\alpha}(0) \frac{s^{2}}{2 !}+\dddot{\alpha}(0) \frac{s^{3}}{3 !}+\ldots,
$$

we can conclude that $\alpha$ is lying in the plane spanned by $\{\dot{\alpha}(0), \ddot{\alpha}(0)\}$. 
Conversely, suppose $\alpha$ is a plane curve. Then there exists points $p$ and $q$ such that $(\alpha(s)-p) \cdot q=0$ for all $s$. Differentiation yields

$$
\dot{\alpha}(s) \cdot q=\ddot{\alpha}(s) \cdot q=0 .
$$

Thus $q$ is always orthogonal to $T=\dot{\alpha}$ and $N=\frac{\ddot{\alpha}}{\kappa_{1}}$. But $B$ is also orthogonal to $T$ and $N$, so, since $B$ has unit length, $B=\frac{q}{\|q\|}$. Thus $\dot{B}=0$, and by definition $\kappa_{2}=0$.

For simpleness, we now distinguish the three possible cases.

Case 1. $g(\ddot{\alpha}, \ddot{\alpha})>0$

The Frenet formulas are given by

$$
\begin{aligned}
& \dot{T}=\kappa_{1} N \\
& \dot{N}=-\kappa_{1} T+\kappa_{2} B \\
& \dot{B}=\kappa_{2} N
\end{aligned}
$$

In this case the curves with constant curvatures are characterized by the following theorem.

Theorem 3.3. For a spacelike curve in $\mathbb{E}_{1}^{3}$, with $g(\ddot{\alpha}, \ddot{\alpha})>0$, we have that

(1) $\kappa_{2}=0$ and $\kappa_{1}=$ constant $>0$ if and only if $\alpha$ is a part of a circle;

(2) $\kappa_{1}=$ constant $>0, \kappa_{2}=$ constant $\neq 0$ and $\left|\kappa_{2}\right|>\kappa_{1}$ if and only if $\alpha$ is a part of a spacelike hyperbolic helix,

$$
\alpha(s)=\frac{1}{K}\left(\kappa_{1} \sinh (\sqrt{K} s), \sqrt{\kappa_{2}^{2} K} s, \kappa_{1} \cosh (\sqrt{K} s)\right)
$$

with $K=\kappa_{2}^{2}-\kappa_{1}^{2}$

(3) $\kappa_{1}=$ constant $>0, \kappa_{2}=$ constant $\neq 0$ and $\left|\kappa_{2}\right|<\kappa_{1}$ if and only if $\alpha$ is a part of a spacelike circular helix,

$$
\alpha(s)=\frac{1}{K}\left(\sqrt{\kappa_{2}^{2} K} s, \kappa_{1} \cos (\sqrt{K} s), \kappa_{1} \sin (\sqrt{K} s)\right)
$$

with $K=\kappa_{1}^{2}-\kappa_{2}^{2}$;

(4) $\kappa_{1}=$ constant $>0, \kappa_{2}=$ constant $\neq 0$ and $\left|\kappa_{2}\right|=\kappa_{1}$ if and only if $\alpha$ can be parameterized by

$$
\alpha(s)=\frac{1}{6}\left(\kappa_{1} \kappa_{2} s^{3},-\kappa_{1}^{2} s^{3}+6 s, 3 \kappa_{1} s^{2}\right) .
$$

\section{Proof.}

(1) Suppose $\kappa_{1}=$ constant $>0$ and $\kappa_{2}=0$. From theorem 3.2. we know that $\alpha$ is a planar curve and from the proof of theorem 2.3. we see that $\alpha(s)$ lies in the plane spanned by $\{\dot{\alpha}(0), \ddot{\alpha}(0)\} . \alpha$ is a spacelike curve and $g(\ddot{\alpha}, \ddot{\alpha})>0$, so we know that $\alpha$ lies in a spacelike plane. Define

$$
\alpha_{0}(s)=\alpha(s)+\frac{1}{\kappa_{1}} N(s) .
$$


Taking the derivative, we get

$$
\dot{\alpha}_{0}(s)=\dot{\alpha}(s)+\frac{1}{\kappa_{1}} \dot{N}(s)=0 .
$$

$\alpha_{0}(s)$ is a constant curve, so $\alpha(s)+\frac{1}{\kappa_{1}} N(s)$ has the same value, say $c$, for all $s$. The distance between $c$ and $\alpha(s)$ is equal to

$$
d(c, \alpha(s))=\|c-\alpha(s)\|=\left\|\frac{N(s)}{\kappa_{1}}\right\|=\frac{1}{\left|\kappa_{1}\right|} .
$$

Thus $\alpha(s)$ is a part of a circle.

Conversely, suppose $\alpha(s)$ is a planar spacelike circle. From theorem 3.2. we know that $\kappa_{2}=0$, and from the proof of theorem 2.3. we see that $\alpha(s)$ lies in the plane spanned by $\{\dot{\alpha}(0), \ddot{\alpha}(0)\}$. $\alpha$ is a spacelike curve and $g(\ddot{\alpha}, \ddot{\alpha})>0$, so we know that $\alpha$ lies in a spacelike plane. Without loss of generality, we can take the plane $x=0$. Thus

$$
\alpha(s)=(0, r \cos (s / r), r \sin (s / r)),
$$

with $r \in \mathbb{R}, r>0$. Calculating $\kappa_{1}$ gives that $\kappa_{1}=\frac{1}{r}=$ constant.

(2) Suppose that $\kappa_{1}=$ constant $>0, \kappa_{2}=$ constant $\neq 0$ and $\left|\kappa_{2}\right|>\kappa_{1}$.

If we take the curve $\alpha_{0}$

$$
\alpha_{0}(s)=\alpha(s)+\frac{\kappa_{1}}{\kappa_{1}^{2}-\kappa_{2}^{2}} N(s)
$$

it's easy to see that this curve is a straight line. The causal character of this curve is determined by

$$
g\left(\dot{\alpha}_{0}, \dot{\alpha}_{0}\right)=\frac{-\kappa_{2}^{2}}{\kappa_{1}^{2}-\kappa_{2}^{2}} .
$$

Since $\alpha_{0}$ is a spacelike straight line, we may assume, without loss of generality, that $\alpha_{0}$ is the Y-axis. From $g\left(\dot{\alpha}_{0}, N\right)=0$, we obtain that

$$
N(s)=-(\sinh (f(s)), 0, \cosh (f(s)))
$$

and

$$
\alpha(s)=\left(\frac{\kappa_{1}}{\kappa_{1}^{2}-\kappa_{2}^{2}} \sinh (f(s)), h(s), \frac{\kappa_{1}}{\kappa_{1}^{2}-\kappa_{2}^{2}} \cosh (f(s))\right) .
$$

Using the Frenet formulas and the fact that $\alpha$ is spacelike, we find that

$$
h(s)=\sqrt{\frac{\kappa_{2}^{2}}{\kappa_{2}^{2}-\kappa_{1}^{2}}} s
$$

and

$$
f(s)=\sqrt{\kappa_{2}^{2}-\kappa_{1}^{2}} s .
$$

$\alpha$ is then a part of a spacelike hyperbolic helix. 
If $\alpha$ is a part of a spacelike hyperbolic helix, then by differentiation and using the Frenet equations we find that $\kappa_{1}$ and $\kappa_{2}$ are constant and satisfy the necessary condition.

(3) Suppose that $\kappa_{1}>\left|\kappa_{2}\right|$ and that both curvatures are constant.

If we take the curve $\alpha_{0}$

$$
\alpha_{0}(s)=\alpha(s)+\frac{\kappa_{1}}{\kappa_{1}^{2}-\kappa_{2}^{2}} N(s)
$$

then it is easy to see that this curve is a straight line. The causal character of this curve is determined by

$$
g\left(\dot{\alpha}_{0}, \dot{\alpha}_{0}\right)=\frac{-\kappa_{2}^{2}}{\kappa_{1}^{2}-\kappa_{2}^{2}} .
$$

Since $\alpha_{0}$ is a timelike straight line, we may assume without loss of generality that $\alpha_{0}$ is the $\mathrm{X}$-axis. From $g\left(\dot{\alpha}_{0}, N\right)=0$, we obtain that

$$
N(s)=-(0, \cos (f(s)), \sin (f(s)))
$$

and

$$
\alpha(s)=\left(h(s), \frac{\kappa_{1}}{\kappa_{1}^{2}-\kappa_{2}^{2}} \cos (f(s)), \frac{\kappa_{1}}{\kappa_{1}^{2}-\kappa_{2}^{2}} \sin (f(s))\right) .
$$

Using the Frenet formulas and the fact that $\alpha$ is spacelike, gives

$$
h(s)=\sqrt{\frac{\kappa_{2}^{2}}{\kappa_{1}^{2}-\kappa_{2}^{2}}} s
$$

and

$$
f(s)=\sqrt{\kappa_{1}^{2}-\kappa_{2}^{2}} s .
$$

In this case $\alpha$ is thus a part of a spacelike circular helix.

Conversely, if $\alpha$ is a part of a spacelike circular helix, then by applying the Frenet equations we get that both curvatures are constant and satisfy the postulated condition.

(4) Suppose that $\kappa_{1}=\left|\kappa_{2}\right|$ with both curvatures constant.

From the Frenet formulas, we have that

$$
\ddot{N}=0 \text {. }
$$

Without loss of generality we may assume that for $s=0, \quad \alpha(s)=(0,0,0)$, $T(0)=(0,1,0), \quad N(0)=(0,0,1)$ and $\quad B(0)=(1,0,0)$. We find that

$$
N(s)=\left(c_{1} s, c_{2} s, c_{3} s+1\right)
$$

with $\forall i \in\{1,2,3\}: c_{i} \in \mathbb{R}$. From the Frenet formulas and after some integrations, we get that

$$
\alpha(s)=\frac{1}{6}\left(c_{1} \kappa_{1} s^{3}, c_{2} \kappa_{1} s^{3}+6 s, c_{3} \kappa_{1} s^{3}+3 \kappa_{1} s^{2}\right) .
$$


By straightforward computations and using again the Frenet formulas, we obtain that $c_{1}=\kappa_{2}, \quad c_{2}=-\kappa_{1}, \quad c_{3}=0 \quad$ which we had to prove. The other part can be proved by taking the first and second derivative of the curve $\alpha$ and by applying the Frenet equations.

Case 2. $g(\ddot{\alpha}, \ddot{\alpha})<0$

The Frenet formulas are given by

$$
\begin{aligned}
& \dot{T}=\kappa_{1} N \\
& \dot{N}=\kappa_{1} T+\kappa_{2} B \\
& \dot{B}=\kappa_{2} N
\end{aligned}
$$

In this case the curves with constant curvatures are characterized by the following theorem.

Theorem 3.4. For a spacelike curve in $\mathbb{E}_{1}^{3}$, with $g(\ddot{\alpha}, \ddot{\alpha})<0$, we have that

(1) $\kappa_{2}=0$ and $\kappa_{1}=$ constant $>0$ if and only if $\alpha$ is a part of an orthogonal hyperbola;

(2) $\kappa_{1}=$ constant $>0$ and $\kappa_{2}=$ constant $\neq 0$ if and only if $\alpha$ is a part of a spacelike hyperbolic helix,

$$
\alpha(s)=\frac{1}{K}\left(\kappa_{1} \cosh (\sqrt{K} s), \sqrt{\kappa_{2}^{2} K} s, \kappa_{1} \sinh (\sqrt{K} s)\right)
$$

with $K=\kappa_{1}^{2}+\kappa_{2}^{2}$.

\section{Proof.}

(1) Suppose $\kappa_{1}=$ constant $>0$ and $\kappa_{2}=0$. From theorem 3.2. we know that $\alpha$ is a planar curve and from the proof of theorem 2.3. we see that $\alpha(s)$ lies in the plane spanned by $\{\dot{\alpha}(0), \ddot{\alpha}(0)\}$. $\alpha$ is a spacelike curve and $g(\ddot{\alpha}, \ddot{\alpha})<0$, so we know that $\alpha$ lies in a timelike plane. Define

$$
\alpha_{0}(s)=\alpha(s)-\frac{1}{\kappa_{1}} N(s) .
$$

Taking the derivative, we get

$$
\dot{\alpha}_{0}(s)=\dot{\alpha}(s)-\frac{1}{\kappa_{1}} \dot{N}(s)=0 .
$$

$\alpha_{0}(s)$ is a constant curve, so $\alpha(s)-\frac{1}{\kappa_{1}} N(s)$ has the same value, say $c$, for all $s$. The distance between $c$ and $\alpha(s)$ is equal to

$$
d(c, \alpha(s))=\|c-\alpha(s)\|=\left\|\frac{-N(s)}{\kappa_{1}}\right\|=\frac{1}{\left|\kappa_{1}\right|} .
$$

Thus $\alpha(s)$ is a part of a orthogonal hyperbola. 
Conversely, suppose $\alpha(s)$ is a part of a planar orthogonal hyperbola. From theorem 3.2. we know that $\kappa_{2}=0$, and from the proof of theorem 2.3. we see that $\alpha(s)$ lies in the plane spanned by $\{\dot{\alpha}(0), \ddot{\alpha}(0)\}$. $\alpha$ is a spacelike curve and $g(\ddot{\alpha}, \ddot{\alpha})<0$, so we know that $\alpha$ lies in a timelike plane. Without loss of generality, we can take the plane $z=0$. Thus

$$
\alpha(s)=(r \sinh (s / r), r \cosh (s / r), 0),
$$

with $r \in \mathbb{R}, r>0$. Calculating $\kappa_{1}$ gives that $\kappa_{1}=\frac{1}{r}=$ constant.

(2) Suppose that $\kappa_{1}=$ constant $>0$ and $\kappa_{2}=$ constant $\neq 0$.

If we take the curve $\alpha_{0}$

$$
\alpha_{0}(s)=\alpha(s)-\frac{\kappa_{1}}{\kappa_{1}^{2}+\kappa_{2}^{2}} N(s),
$$

it's easy to calculate that $\ddot{\alpha}_{0}(s)=0$, so this curve is a straight line. The causal character of this curve is determined by

$$
g\left(\dot{\alpha}_{0}, \dot{\alpha}_{0}\right)=\frac{\kappa_{2}^{2}}{\kappa_{1}^{2}+\kappa_{2}^{2}} .
$$

Since $\alpha_{0}$ is a spacelike straight line, we may assume, without loss of generality, that $\alpha_{0}$ is the Y-axis. From $g\left(\dot{\alpha}_{0}, N\right)=0$, we obtain that

$$
N(s)=(\cosh (f(s)), 0, \sinh (f(s)))
$$

and

$$
\alpha(s)=\left(\frac{\kappa_{1}}{\kappa_{1}^{2}+\kappa_{2}^{2}} \cosh (f(s)), h(s), \frac{\kappa_{1}}{\kappa_{1}^{2}+\kappa_{2}^{2}} \sinh (f(s))\right) .
$$

Using the Frenet formulas and the fact that $\alpha$ is spacelike, we find that

$$
h(s)=\sqrt{\frac{\kappa_{2}^{2}}{\kappa_{2}^{2}+\kappa_{1}^{2}}} s
$$

and

$$
f(s)=\sqrt{\kappa_{2}^{2}+\kappa_{1}^{2}} s .
$$

$\alpha$ is then a part of a spacelike hyperbolic helix.

If $\alpha$ is a part of a spacelike hyperbolic helix, then by differentiation and using the Frenet equations we find that $\kappa_{1}$ and $\kappa_{2}$ are constant.

Case 3. $g(\ddot{\alpha}, \ddot{\alpha})=0$

$\alpha$ is a pseudo null curve and the Frenet formulas are given by

$$
\begin{aligned}
\dot{T} & =\kappa_{1} N \\
\dot{N} & =\kappa_{2} N \\
\dot{B} & =-\kappa_{1} T-\kappa_{2} B
\end{aligned}
$$

where the " curvature " $\kappa_{1}$ can only take two values; 0 , if $\alpha$ is a spacelike straight line, or 1 in all other cases. In this case the curves with constant curvatures are characterized by the following theorem. 
Theorem 3.5. All pseudo null spacelike curves in $\mathbb{E}_{1}^{3}$ with constant curvatures can be classified as

(1) $\kappa_{1}=0$ if and only if $\alpha$ is a part of a spacelike straight line;

(2) $\kappa_{1}=1$ and $\kappa_{2}=0$ if and only if $\alpha$ is a part of a planar curve with parameterisation

$$
\alpha(s)=\left(\frac{s^{2}}{2}, s, \frac{s^{2}}{2}\right) ;
$$

(3) $\kappa_{1}=1$ and $\kappa_{2}=$ constant $\neq 0$ if and only if $\alpha$ is a part of a planar curve with parameterisation

$$
\alpha(s)=\frac{1}{\kappa_{2}^{2}}\left(\cosh \left(\kappa_{2} s\right)+\sinh \left(\kappa_{2} s\right), \kappa_{2}^{2} s, \cosh \left(\kappa_{2} s\right)+\sinh \left(\kappa_{2} s\right)\right)
$$

\section{Proof.}

(1) If $\kappa_{1}=0$, then by definition $\alpha$ is a straight line. Conversely, if $\alpha$ is a straight line then $\ddot{\alpha}=0$ and thus $\kappa_{1}=0$.

(2) If $\kappa_{1}=1$ and $\kappa_{2}=0$, we can use the Frenet formulas and the Maclaurin serie of $\alpha$ to get that

$$
\alpha(s)=\alpha(0)+T(0) s+N(0) \frac{s^{2}}{2} .
$$

From this equation, we see that $\alpha$ is a planar curve. Without loss of generality, we may assume that

$$
\alpha(0)=(0,0,0), \quad T(0)=(0,1,0) \text { and } \quad N(0)=(1,0,1) .
$$

We find that

$$
\alpha(s)=\left(\frac{s^{2}}{2}, s, \frac{s^{2}}{2}\right) .
$$

If $\alpha(s)$ has the above parameterisation, then $\dddot{\alpha}(s)=0$. This means that $\dot{N}(s)=0$. Thus from the Frenet formulas follows that $\kappa_{2}=0$.

(3) If $\kappa_{1}=1$ and $\kappa_{2}=$ constant $\neq 0$, then

$$
\alpha_{0}(s)=\alpha(s)-\frac{1}{\kappa_{2}^{2}} N(s)
$$

is a spacelike straight line. Without loss of generality, we may assume that $\alpha_{0}$ is the Y-axis. We know that $N(s)$ is a null vector perpendicular to $\dot{\alpha}_{0}$, so it can be written as

$$
N(s)=n(s)(1,0,1) .
$$

After some calculations, we find that

$$
\dot{n}(s)=\kappa_{2} n(s) .
$$

Solving this differential equation and using that

$$
\alpha(s)=\alpha_{0}(s)+\frac{1}{\kappa_{2}^{2}} N(s),
$$

we get what we had to prove.

By derivating $\alpha$ and applying the Frenet formulas, we find that $\kappa_{2}$ is constant. 


\subsection{Timelike curves.}

The Frenet formulas are given by

$$
\begin{aligned}
\dot{T} & =\kappa_{1} N \\
\dot{N} & =\kappa_{1} T+\kappa_{2} B \\
\dot{B} & =-\kappa_{2} N
\end{aligned}
$$

Timelike curves with constant curvatures are characterized by the following theorem.

Theorem 3.6. All timelike curves in $\mathbb{E}_{1}^{3}$ with constant curvatures can be classified as

(1) $\kappa_{1}=0$ if and only if $\alpha$ is a part of a timelike straight line;

(2) $\kappa_{2}=0$ if and only if $\alpha$ is a planar timelike curve;

(3) $\kappa_{2}=0$ and $\kappa_{1}=$ constant $>0$ if and only if $\alpha$ is a part of a orthogonal hyperbola;

(4) $\kappa_{1}=$ constant $>0, \kappa_{2}=$ constant $\neq 0$ and $\left|\kappa_{2}\right|>\kappa_{1}$ if and only if $\alpha$ is a part of a timelike circular helix,

$$
\alpha(s)=\frac{1}{K}\left(\sqrt{\kappa_{2}^{2} K} s, \kappa_{1} \cos (\sqrt{K} s), \kappa_{1} \sin (\sqrt{K} s)\right)
$$

with $K=\kappa_{2}^{2}-\kappa_{1}^{2}$;

(5) $\kappa_{1}=$ constant $>0, \kappa_{2}=$ constant $\neq 0$ and $\left|\kappa_{2}\right|<\kappa_{1}$ if and only if $\alpha$ is a part of a timelike hyperbolic helix,

$$
\alpha(s)=\frac{1}{K}\left(\kappa_{1} \sinh (\sqrt{K} s), \sqrt{\kappa_{2}^{2} K} s, \kappa_{1} \cosh (\sqrt{K} s)\right)
$$

with $K=\kappa_{1}^{2}-\kappa_{2}^{2}$

(6) $\kappa_{1}=$ constant $>0, \kappa_{2}=$ constant $\neq 0$ and $\left|\kappa_{2}\right|=\kappa_{1}$ if and only if $\alpha$ can be parameterized by

$$
\alpha(s)=\frac{1}{6}\left(\kappa_{1}^{2} s^{3}+6 s, 3 \kappa_{1} s^{2}, \kappa_{1} \kappa_{2} s^{3}\right) .
$$

\section{Proof.}

Same methods as in the proofs of theorem 3.1., 3.2. and 3.3.

\subsection{Null curves.}

The Frenet formulas are given by

$$
\begin{aligned}
\dot{T} & =\kappa_{1} N \\
\dot{N} & =\kappa_{2} T-\kappa_{1} B \\
\dot{B} & =-\kappa_{2} N
\end{aligned}
$$

where the " curvature" $\kappa_{1}$ can take only two values; 0 , when $\alpha$ is a straight null line, or 1 in all other cases. Null curves with constant curvatures are characterized by the following theorem. 
Theorem 3.7. All null curves in $\mathbb{E}_{1}^{3}$ with constant curvatures can be classified as

(1) $\kappa_{1}=0$ if and only if $\alpha$ is a part of a null straight line;

(2) $\kappa_{1}=1$ and $\kappa_{2}=0$ if and only if $\alpha$ is a part of the null cubic,

$$
\alpha(s)=\frac{1}{6 \sqrt{2}}\left(6 s+s^{3}, 3 \sqrt{2} s^{2}, 6 s-s^{3}\right)
$$

(3) $\kappa_{1}=1$ and $\kappa_{2}>0$ if and only if $\alpha$ is a part of a null circular helix,

$$
\alpha(s)=\frac{1}{K^{2}}(K s, \cos (K s), \sin (K s))
$$

with $K=\sqrt{2 \kappa_{2}}$;

(4) $\kappa_{1}=1$ and $\kappa_{2}<0$ if and only if $\alpha$ is a part of a null hyperbolic helix,

$$
\alpha(s)=\frac{1}{K^{2}}(\sinh (K s), K s, \cosh (K s))
$$

with $K=\sqrt{-2 \kappa_{2}}$.

Proof. This proof is very similar with previous proofs, so we give only the most deviating parts.

(2) Suppose that $\kappa_{2}=0$. Then the Maclaurin serie of $\alpha$ can be written as

$$
\alpha(s)=\alpha(0)+T(0) s+N(0) \frac{s^{2}}{2}-B(0) \frac{s^{3}}{6}
$$

with $\{T, N, B\}$ the Frenet frame of $\alpha$. In this case, $T$ and $B$ are null vectors, such that $g(T, B)=1$, and $N$ is a unit spacelike vector. Without loss of generality, we may assume that

$$
T(0)=\frac{1}{\sqrt{2}}(1,0,1), \quad N(0)=(0,1,0), \quad B(0)=\frac{1}{\sqrt{2}}(-1,0,1) .
$$

This gives us the desired result.

(3) and (4) If $\kappa_{2} \neq 0$, then

$$
\alpha_{0}(s)=\alpha(s)-\frac{1}{2 \kappa_{2}} N(s)
$$

is a straight line. The causal character of this line depends on the sign of $\kappa_{2}$. If $\kappa_{2}<0, \quad \alpha_{0}$ is a timelike straight line, say the $\mathrm{X}$-axis. In the other case, $\alpha_{0}$ is a spacelike line, say the Y-axis. The spacelike unit vector $N(s)$ is, in both cases, perpendicular to $\dot{\alpha}_{0}$. This means that $N(s)$ takes a well-determined form. From the definition of $\alpha_{0}$, we see that $\alpha$ is known when $N$ is known. 


\section{§4. CURVES OF RESTRICTED TYPE}

\subsection{Introduction.}

A submanifold $N^{n}$ in Euclidean space $\mathbb{E}^{m}$ is said to be of restricted type (R.T.) if for each tangent vector $X$ at any point $\mathrm{p}$ of $N^{n}$

$$
A_{H}(X)=(M X)^{T}
$$

where $A_{H}$ denotes the shape operator or Weingarten map of $N^{n}$ at $\mathrm{p}$ with respect to the mean curvature vector $H,()^{T}$ denotes the tangential component and $M \in \mathbb{R}^{m \times m}$ is a fixed endomorphism of the ambient space [CDVV2]. The following theorem [CDVV2] gives a complete classification of the curves of restricted type in the Euclidean plane $\mathbb{E}^{2}$.

Theorem 4.1. Up to rigid motions of $\mathbb{E}^{2}$, a curve in $\mathbb{E}^{2}$ is of restricted type if and only if it is anen portion of one of the following plane curves :

(1) a line;

(2) a circle;

(3) a curve with equation : $\cos (c x)=e^{-c y}$, where $c \neq 0$;

(4) a curve with equation: $a \sin ^{2}(\sqrt{c} x)+b \sinh ^{2}(\sqrt{c} y)=c$, where $a>b>0$, $c=a-b$

(5) a curve with equation : $a \sin ^{2}(\sqrt{c} x)-b \cosh ^{2}(\sqrt{c} y)=c$, where $a>0>b$, $c=a-b$.

Notice that the equations in (4) and (5) resp. can be written as

$$
\sqrt{b} \cosh y \pm \sqrt{a} \cos x=0
$$

and

$$
\sqrt{-b} \sinh y \pm \sqrt{a} \cos x=0 .
$$

We will give a classification of all plane curves of restricted type in the 2-dimensional Minkowski space $\mathbb{E}_{1}^{2}$.

Let $\beta(s)$ be a curve in $\mathbb{E}_{1}^{2}, \quad \mathbb{R}^{2}$ with the non-degenerate metric $g=d x_{1}^{2}-d x_{2}^{2}$. We will consider only two cases, namely the spacelike and the timelike curves of restricted type in $\mathbb{E}_{1}^{2}$, the only null curves in $\mathbb{E}_{1}^{2}$ being straight lines.

We define a curve $\beta$, parametrized by arclength $s$, to be of restricted type in $\mathbb{E}_{1}^{2}$ if there exist a fixed endomorphism $M \in \mathbb{R}^{2 \times 2}$ such that

$$
A_{H}(X)=(M X)^{T}
$$

Let $\dot{\beta}(s)=T(s)$, then $(4.2)$ is equivalent with

$$
g\left(A_{H}(T), T\right)=g(M T, T) .
$$


Using the Frenet formulas for $\beta$ and the formula of Gauss, we get that

$$
\ddot{\beta}=\dot{T}=\kappa N=D_{T} T=h(T, T) .
$$

From the definition of $H, H=\varepsilon_{T} h(T, T)$ with $\varepsilon_{T}=g(T, T)$, we find that

$$
H=\varepsilon_{T} \kappa N .
$$

This expression for the mean curvature vector $H$ of $\beta$, leads to

$$
g\left(A_{H} T, T\right)=\varepsilon_{T} \kappa g\left(A_{N} T, T\right)=\varepsilon_{T} \kappa g\left(D_{T} T, N\right)=\varepsilon_{T} \kappa g(h(T, T), N)=\varepsilon_{T} \kappa^{2} g(N, N) .
$$

Condition (4.2) for a planar curve $\beta$ in $\mathbb{E}_{1}^{2}$ turns out to be equivalent with,

$$
-\kappa^{2}=g(M T, T)
$$

where $\kappa$ denotes the curvature from the Frenet formulas of $\beta$ and $T=\dot{\beta}$.

In a Minkowski space it is not possible to diagonalize every symmetric, linear operator. But there exists another classification for self-adjoint operators, which makes it possible to say something about the form of $M$. From the symmetry of $A_{H}$ in definition (4.1), we can convert $M$ into a self-adjoint operator by replacing $M$ by

$$
\tilde{M}=\frac{1}{2}\left(M+\varepsilon M^{t} \varepsilon\right)
$$

with

$$
\varepsilon=\left(\begin{array}{cc}
-1 & 0 \\
0 & 1
\end{array}\right)
$$

From the classificationtheorem for self-adjoint operators in a Lorentz space, due to Petrov [Pe], we know that $M$ takes one of the following forms :

$$
M=\left(\begin{array}{ll}
a & 0 \\
0 & b
\end{array}\right)
$$

with respect to an orthonormal basis;

$$
M=\left(\begin{array}{cc}
a & b \\
-b & a
\end{array}\right),
$$

with respect to an orthonormal basis and $b \neq 0$;

$$
M=\left(\begin{array}{ll}
a & 0 \\
1 & a
\end{array}\right),
$$

with respect to a pseudo-orthonormal basis $u_{1}, u_{2}$; $g\left(u_{1}, u_{1}\right)=g\left(u_{2}, u_{2}\right)=0 ; \quad g\left(u_{1}, u_{2}\right)=-1 ;$ where $a, b \in \mathbb{R}$.

Using these representations of $M$, we can determine all the curves of restricted type in $\mathbb{E}_{1}^{2}$. We first make the following observation. 
Lemma 4.2. If $\beta$ is a curve of restricted type in $\mathbb{E}_{1}^{2}$, then the reflection of $\beta$ w.r.t. one of the bisectors is also a curve of restricted type in $\mathbb{E}_{1}^{2}$.

Proof. We'll give the proof for a spacelike curve $\beta$, the proof for a timelike curve is completely similar.

Suppose that $\beta$ is a spacelike curve of restricted type in $\mathbb{E}_{1}^{2}$. This means that there exists a matrix $M$ such that

$$
-\kappa^{2}=g(M T, T)
$$

with $\dot{\beta}=T=(\cosh (\theta(s)), \sinh (\theta(s)))$ and

$$
M=\left(\begin{array}{cc}
a & c \\
-c & b
\end{array}\right)
$$

with respect to an orthonormal basis. This is equivalent with

$$
\kappa^{2}=-a \cosh ^{2}(\theta(s))-2 c \sinh (\theta(s)) \cosh (\theta(s))+b \sinh ^{2}(\theta(s)) .
$$

Let $\beta_{1}$ be the reflection of $\beta$ with respect to the first bisector. We must prove that $\beta_{1}$ is also of restricted type.

Knowing that $\dot{\beta}_{1}=T_{1}=(\sinh (\theta(s)), \cosh (\theta(s)))$ and that $\kappa=\kappa_{1}=\dot{\theta}$, we have that

$$
\kappa_{1}^{2}=g\left(M_{1} T_{1}, T_{1}\right)
$$

with

$$
M_{1}=\left(\begin{array}{cc}
-b & c \\
-c & -a
\end{array}\right) .
$$

This means that $\beta_{1}$ is a timelike curve of restricted type .

Let $\beta_{2}$ be the reflection of $\beta$ with respect to the second bisector. We'll prove that also $\beta_{2}$ is of restricted type in $\mathbb{E}_{1}^{2}$.

Knowing that $\dot{\beta}_{2}=T_{2}=(-\sinh (\theta(s)),-\cosh (\theta(s)))$ and that $\kappa_{2}=-\kappa=-\dot{\theta}$, we have that

$$
\kappa_{2}^{2}=g\left(M_{2} T_{2}, T_{2}\right)
$$

with

$$
M_{2}=\left(\begin{array}{cc}
-b & c \\
-c & -a
\end{array}\right)
$$

We find that $\beta_{2}$ is a timelike curve of restricted type in $\mathbb{E}_{1}^{2}$.

Remark that if we know all spacelike curves of restricted type in $\mathbb{E}_{1}^{2}$, we find by reflection w.r.t. one of the bisectors also all the timelike curves of restricted type in $\mathbb{E}_{1}^{2}$.

\subsection{Classification.}

Considering the remark above, it is enough to classify all the spacelike curves of restricted type in $\mathbb{E}_{1}^{2}$. 
Theorem 4.3. Up to rigid motions of $\mathbb{E}_{1}^{2}$, a spacelike curve in $\mathbb{E}_{1}^{2}$ is of restricted type if and only if it is an open portion of one of the following plane curves :

(1) a straight line;

(2) an orthogonal hyperbola;

Curves with equations:

(3) $\sinh (c y)=e^{c x}, \quad c \neq 0$;

(4) $\cosh (c x)=e^{c y}, \quad c \neq 0$;

(5) $-a \cosh ^{2}(\sqrt{c} x)+b \cosh ^{2}(\sqrt{c} y)=c, \quad b>a>0, \quad c=b-a$;

(6) $a \sinh ^{2}(\sqrt{c} x)+b \cosh ^{2}(\sqrt{c} y)=c, \quad b>0>a, \quad c=b-a$;

(7) $a \sinh ^{2}(\sqrt{c} x)-b \sinh ^{2}(\sqrt{c} y)=c, \quad 0>b>a, \quad c=b-a$;

(8) $a \sin ^{2}(\sqrt{c} x)-b \sin ^{2}(\sqrt{c} y)=c, \quad 0>a>b, \quad c=a-b$;

(9) $[\cos (\sqrt{2 b}(x+y))-d] \cdot[\cosh (\sqrt{2 b}(x-y))+d]=c^{2}, \quad b>0, \quad c=\frac{b}{\sqrt{a^{2}+b^{2}}}$, $d=\frac{a}{\sqrt{a^{2}+b^{2}}}$;

$d=\frac{a}{\sqrt{a^{2}+b^{2}}}$;

(10) $[\cosh (\sqrt{-2 b}(x+y))+d] \cdot[\cos (\sqrt{-2 b}(x-y))-d]=c^{2}, \quad b<0, \quad c=\frac{b}{\sqrt{a^{2}+b^{2}}}$,
(11) $e^{x}=\frac{1}{2 \sqrt{y}}$
(12) $y=c \tanh x, \quad c<0$;
(13) $y=c \operatorname{coth} x, \quad c>0$.

\section{Proof.}

From definition (4.3) we know that $\beta$ is a spacelike curve of R.T. if and only if there exists an endomorphism $M$ such that

$$
-\kappa^{2}=g(M T, T)
$$

with $T=\dot{\beta}, \quad g(T, T)=1$.

\section{Case 1.}

Assume that

$$
M=\left(\begin{array}{ll}
a & 0 \\
0 & b
\end{array}\right),
$$

with respect to an orthonormal basis and $a, b \in \mathbb{R}$. Let

$$
T(s)=(\cosh (\theta(s)), \sinh (\theta(s))) \text {. }
$$

Then

$$
\kappa^{2}(s)=\left(\frac{d \theta}{d s}\right)^{2},
$$

such that (4.4) becomes

$$
\left(\frac{d \theta}{d s}\right)^{2}=-a \cosh ^{2}(\theta)+b \sinh ^{2}(\theta) .
$$


This condition implies the following cases.

1.1. $a=b=0$. In this case, the curve is a portion of a straight line.

1.2. $a=b<0$. Here $\beta$ is a part of an orthogonal hyperbola,

$$
\beta(s)=\left(\alpha \sinh \left(\frac{s}{\alpha}\right), \alpha \cosh \left(\frac{s}{\alpha}\right)\right)
$$

with $\alpha= \pm \frac{1}{\sqrt{-a}}$.

1.3. $a \neq b$. We can divide $\beta$ into pieces such that on each piece, $\frac{d \theta}{d s} \neq 0$. We then can compute the inverse function $s(\theta)$. Let $\gamma(\theta)=\beta(s(\theta))$ be the reparametrization of $\beta$ in terms of $\theta$. Then

$$
\frac{d \gamma}{d \theta}= \pm \frac{1}{\sqrt{-a \cosh ^{2}(\theta)+b \sinh ^{2}(\theta)}}(\cosh (\theta), \sinh (\theta))
$$

1.3.1. $a=0, \quad b>0$.

We can integrate (4.5) immediatly and obtain, up to translations

$$
\gamma(\theta)= \pm \frac{1}{\sqrt{b}}(\ln (\sinh (\theta)), \theta)
$$

such that $\gamma$ is of form (3).

1.3.2. $a<0, \quad b=0$.

We get a curve of form (4);

$$
\gamma(\theta)= \pm \frac{1}{\sqrt{-a}}(\theta, \ln (\cosh (\theta)))
$$

1.3.3. $b>a>0$.

From (3.2), $\theta$ is restricted to

$$
\sinh (\theta)>\sqrt{\frac{a}{b-a}}, \quad \cosh (\theta)>\sqrt{\frac{b}{b-a}}
$$

and we have that

$$
\gamma(\theta)= \pm \frac{1}{\sqrt{b-a}}\left(\operatorname{arccosh}\left(\sqrt{\frac{b-a}{a}} \sinh (\theta)\right), \operatorname{arccosh}\left(\sqrt{\frac{b-a}{b}} \cosh (\theta)\right)\right) .
$$

It is clear that $\gamma$ takes form (5).

1.3.4. $b>0>a$.

After some computations, we get a curve from form (6),

$$
\gamma(\theta)= \pm \frac{1}{\sqrt{b-a}}\left(\operatorname{arcsinh}\left(\sqrt{\frac{b-a}{-a}} \sinh (\theta)\right), \operatorname{arccosh}\left(\sqrt{\frac{b-a}{b}} \cosh (\theta)\right)\right) .
$$


1.3.5. $0>b>a$.

In this case $\gamma$ takes form (7),

$$
\gamma(\theta)= \pm \frac{1}{\sqrt{b-a}}\left(\operatorname{arcsinh}\left(\sqrt{\frac{b-a}{-a}} \sinh (\theta)\right), \operatorname{arcsinh}\left(\sqrt{\frac{b-a}{-b}} \cosh (\theta)\right)\right)
$$

1.3.6. $0>a>b$.

We must restrict $\theta$ to

$$
|\theta| \leq \operatorname{arcsinh}\left(\sqrt{\frac{-a}{a-b}}\right)
$$

and we have a curve of form (8),

$$
\gamma(\theta)= \pm \frac{1}{\sqrt{b-a}}\left(\arcsin \left(\sqrt{\frac{a-b}{-a}} \sinh (\theta)\right), \arcsin \left(\sqrt{\frac{a-b}{-b}} \cosh (\theta)\right)\right) .
$$

\section{Case 2.}

Assume that

$$
M=\left(\begin{array}{cc}
a & b \\
-b & a
\end{array}\right),
$$

with respect to an orthonormal basis and $a \in \mathbb{R}, \quad b \in \mathbb{R}_{0}$. From (4.4) and $T$ being the same as in the previous case, we get that

$$
\left(\frac{d \theta}{d s}\right)^{2}=-2 b \sinh (\theta) \cosh (\theta)-a
$$

must be satisfied.

2.1. $b>0$.

For

$$
\sinh (\theta) \cosh (\theta)<\frac{-a}{2 b}
$$

or equivalently

$$
\theta<\frac{1}{2} \operatorname{arcsinh}\left(\frac{-a}{b}\right)
$$

we have that

$$
\gamma(\theta)= \pm \frac{1}{2 \sqrt{2 b}}\left(\arccos \left(f_{1}(\theta)\right)+\operatorname{arccosh}\left(f_{2}(\theta)\right), \arccos \left(f_{1}(\theta)\right)-\operatorname{arccosh}\left(f_{2}(\theta)\right)\right)
$$

with

$$
\begin{gathered}
f_{1}(\theta)=c e^{2 \theta}+d, \quad f_{2}(\theta)=c e^{-2 \theta}-d, \\
c=\frac{b}{\sqrt{a^{2}+b^{2}}}, \quad d=\frac{a}{\sqrt{a^{2}+b^{2}}} .
\end{gathered}
$$

We see that $\gamma$ is of form (9). 
2.2. $b<0$.

For

$$
\sinh (\theta) \cosh (\theta)>\frac{-a}{2 b}
$$

or equivalently

$$
\theta>\frac{1}{2} \operatorname{arcsinh}\left(\frac{-a}{b}\right)
$$

we have that

$$
\gamma(\theta)= \pm \frac{1}{2 \sqrt{-2 b}}\left(\operatorname{arccosh}\left(f_{1}(\theta)\right)+\arccos \left(f_{2}(\theta)\right), \operatorname{arccosh}\left(f_{1}(\theta)\right)-\arccos \left(f_{2}(\theta)\right)\right),
$$

with

$$
\begin{gathered}
f_{1}(\theta)=c e^{2 \theta}+d, \quad f_{2}(\theta)=c e^{-2 \theta}-d, \\
c=\frac{-b}{\sqrt{a^{2}+b^{2}}}, \quad d=\frac{-a}{\sqrt{a^{2}+b^{2}}} .
\end{gathered}
$$

We see that $\gamma$ is of form (10).

Case 3.

Assume that

$$
M=\left(\begin{array}{ll}
a & 0 \\
1 & a
\end{array}\right),
$$

with respect to a pseudo-orthonormal basis $\left(u_{1}, u_{2}\right)$, such that

$$
g\left(u_{1}, u_{1}\right)=g\left(u_{2}, u_{2}\right)=0, \quad g\left(u_{1}, u_{2}\right)=-1 .
$$

With respect to $\left(u_{1}, u_{2}\right)$,

$$
T(s)=\left(\theta(s), \frac{-1}{2 \theta(s)}\right)
$$

with $\theta(s)>0$.

From (4.4) we get the following condition

$$
\kappa^{2}=\theta^{2}-a
$$

with $\kappa$ the curvature of the curve $\beta$. Equivalently, we have

$$
\left(\frac{d \theta}{d s}\right)^{2}=\theta^{2}\left(\theta^{2}-a\right) .
$$

After a reparameterization $\gamma(\theta)=\beta(s(\theta))$, the condition above becomes

$$
\frac{d \gamma}{d \theta}= \pm \frac{1}{\sqrt{\theta^{2}\left(\theta^{2}-a\right)}}\left(\theta(s), \frac{-1}{2 \theta(s)}\right) .
$$

3.1. $a=0$. 
Integrating implies that, for $\theta>0$,

$$
\gamma(\theta)= \pm\left(\ln (\theta), \frac{1}{2 \theta^{2}}\right)
$$

with respect to a pseudo-orthonormal basis, so $\gamma$ is if form (11).

3.2. $a>0$.

For $\theta>\sqrt{a}$, and with respect to a pseudo-orthonormal basis, we have

$$
\gamma(\theta)= \pm\left(\operatorname{arccosh}\left(\frac{\theta}{\sqrt{a}}\right), \frac{-1}{2 a} \tanh \left(\operatorname{arccosh}\left(\frac{\theta}{\sqrt{a}}\right)\right)\right) \text {. }
$$

This is clearly a curve of type (12).

3.3. $a<0$.

In this case $\gamma$ is of type (13)

$$
\gamma(\theta)= \pm\left(\operatorname{arcsinh}\left(\frac{\theta}{\sqrt{-a}}\right), \frac{-1}{2 a} \operatorname{coth}\left(\operatorname{arcsinh}\left(\frac{\theta}{\sqrt{-a}}\right)\right)\right)
$$

with respect to a pseudo-orthonormal basis. 


\section{$\S 5$. CURVES OF FINITE TYPE}

\subsection{Introduction.}

The notion of submanifold of finite type was introduced by B.Y. Chen see [Ch1]. A submanifold $M^{n}$ of an Euclidean space $\mathbb{E}^{n+p}$ is said to be of finite type, if each component of its position vector field $X$ can be written as a finite sum of eigenfunctions of the Laplacian $\Delta$ of $M^{n}$. This means that

$$
X=X_{0}+\sum_{i=1}^{k} X_{i}
$$

where $X_{0}$ is a constant vector and $\Delta X=\lambda_{i} X_{i}$ for $i=1, . ., k$ and $\lambda_{i} \in \mathbb{R}$. If in particular all eigenvalues $\left\{\lambda_{1}, \lambda_{2}, . ., \lambda_{k}\right\}$ are mutually different, then $M^{n}$ is said to be of $k$-type. If one of the $\lambda$ 's is equal to zero, we say that $M^{n}$ is of null $k$-type.

Finite type curves in Euclidean space where studied intensively ([Ch1],[CDDVV], $[\mathrm{CDV}])$. We give some of the results.

* A closed curve $\alpha$ in $\mathbb{E}^{m}$ is of finite Chen type if and only if the Fourier series expansion of the periodic coordinate functions of $\alpha(s)$ with respect to the arc length $s$ is finite.

* Circles are the only closed curves of 1 - type in $\mathbb{E}^{m}$, and the only non-closed ones are straight lines. Moreover, B.y. Chen proved that amongst all closed curves in a Euclidean plane $\mathbb{E}^{2}$, circles are the only ones of finite type [C]. The only non-closed plane curve of finite type is the straight line [CDVV].

* In higher dimensional Euclidean space however, the situation is drastically different: for instance, in Euclidean space $\mathbb{E}^{3}$ for every $k \in \mathbb{N}$ there do exist closed curves of $k$-type [CDDVV].

* A full classification of all closed 2-type curves in $\mathbb{E}^{m}$ can be found in [CDV]. The classification of all 2-type curves in $\mathbb{E}^{m}$ is given in the following theorem [DPVV].

Classificationtheorem. Let $\alpha:[0, L] \rightarrow \mathbb{E}^{n}$ be a curve of 2-type which lies fully in $\mathbb{E}^{n}$. Then either $n=3$ and $\alpha$ is a circular helix or congruent to

$$
\alpha(s)=a\left((12 b)^{\frac{1}{2}} \sin (p s),-b \cos (p s)+\cos (3 p s),-b \sin (p s)+\sin (3 p s)\right),
$$

where $a=\frac{1}{p}(b+3)^{-1}$ and $b \in \mathbb{R}_{0}^{+}$; or else $n=4$ and $\alpha$ is a $W$-curve or congruent to

$$
\begin{gathered}
\alpha(s)=a\left((12 d)^{\frac{1}{2}} \sin (p(s-\theta)),(12 b)^{\frac{1}{2}} \sin (p s),\right. \\
-d \cos (p(s+2 \theta))-b \cos (p s)+\cos (3 p s),-d \sin (p(s+2 \theta))-b \sin (p s)+\sin (3 p s)),
\end{gathered}
$$

where $b, d \in \mathbb{R}_{0}^{+}, \theta \in \mathbb{R}$ and $a=\frac{1}{p}\left[(b+d+3)^{2}-4 b d \sin ^{2} \theta\right]^{\frac{1}{2}}$.

We recall that a curve is called a W-curve if its Frenet curvatures are constant. A $\mathrm{W}$-curve is given by

$$
\alpha(s)=\alpha_{0}+a s e_{0}+\sum_{i=1}^{k} r_{i}\left(\cos \left(a_{i} s\right) e_{2 i-1}+\sin \left(a_{i} s\right) e_{2 i}\right),
$$


where $\left\{e_{0}, e_{1}, e_{2}, \ldots, e_{2 k}\right\}$ is an orthonormal basis of $\mathbb{E}^{2 k+1}$, the numbers $a_{i}$ are positive and mutually different and satisfy $a^{2}+\sum_{i=1}^{k}\left(r_{i} a_{i}\right)^{2}=1$. If $a=0$ and $a_{i}=\frac{1}{r} p_{i}$, with $p_{i} \in \mathbb{N}$, then the curve is closed. Moreover, the curve $\alpha$ lies fully in $\mathbb{E}^{2 k+1}$ if $a \neq 0$ and fully in $\mathbb{E}^{2 k}$ if $a=0$. In the latter case, $\alpha$ lies on a hypersphere of $\mathbb{E}^{2 k}$.

It is natural to do the same in Minkowski space. Consider a curve $\alpha$ in the ndimensional Minkowski space $\mathbb{E}_{1}^{n}$ parameterized by the arclength (we exclude nullcurves). We know that the Laplace operator $\Delta$ of $\alpha$ is given by

$$
\Delta= \pm \frac{d^{2}}{d s^{2}}
$$

and the eigenfunctions of $\Delta$ are $\cos (a s), \sin (a s), \cosh (a s), \sinh (a s)$ and $s$. Following the definition of Chen we find that a finite type curve $\alpha$ in $\mathbb{E}_{1}^{n}$ can be written as

$$
\alpha(s)=a_{0}+b_{0} s+\sum_{t=1}^{k_{1}}\left[a_{t} \cos \left(p_{t} s\right)+b_{t} \sin \left(p_{t} s\right)\right]+\sum_{t=1}^{k_{2}}\left[c_{t} \cosh \left(q_{t} s\right)+d_{t} \sinh \left(q_{t} s\right)\right]
$$

with $a_{i}, b_{i} \in \mathbb{R}^{n} ; \quad 0<p_{1}<p_{2}<. .<p_{k_{1}}$ and $0<q_{1}<q_{2}<. .<q_{k_{2}}$.

\subsection{Curves of finite type in $\mathbb{E}_{1}^{2}$.}

Theorem 5.1. Every curve of finite type in $\mathbb{E}_{1}^{2}$ is of 1-type, and hence a part of an orthogonal hyperbola or a part of a straight line.

Proof. Let $\alpha$ be a curve of finite type in $\mathbb{E}_{1}^{2}$, parameterised by arc length $\mathrm{s}$, then $\alpha$ can be written as (5.1). Expressing that $g(\dot{\alpha}, \dot{\alpha})= \pm 1$ we obtain:

$$
2 B_{0}+\sum_{i=1}^{k_{1}} p_{i}^{2} A_{i i}-\sum_{i=1}^{k_{2}} q_{i}^{2} \overline{C_{i i}}= \pm 1
$$

$$
4 \sum_{\substack{i=1 \\ p_{i}=l}}^{k_{1}} p_{i} B_{i}-\sum_{\substack{i=1 \\ 2 p_{i}=l}}^{k_{1}} p_{i}^{2} \overline{A_{i i}}-2 \sum_{\substack{j, i=1 \\ i>j \\ p_{i}+p_{j}=l}}^{k_{1}} p_{i} p_{j} \overline{A_{i j}}+2 \sum_{\substack{j, i=1 \\ i>j \\ p_{i}-p_{j}=l}}^{k_{1}} p_{i} p_{j} A_{i j}=0
$$

$$
4 \sum_{\substack{i=1 \\ p_{i}=l}}^{k_{1}} p_{i} A_{i}+\sum_{\substack{i=1 \\ 2 p_{i}=l}}^{k_{1}} p_{i}^{2} D_{i i}-2 \sum_{\substack{j, i=1 \\ i>j \\ p_{i}+p_{j}=l}}^{k_{1}} p_{i} p_{j} D_{i j}+2 \sum_{\substack{j, i=1 \\ i>j \\ p_{i}-p_{j}=l}}^{k_{1}} p_{i} p_{j} \overline{D_{i j}}=0
$$

$$
4 \sum_{\substack{i=1 \\ q_{i}=l}}^{k_{2}} q_{i} D_{i}+\sum_{\substack{i=1 \\ 2 q_{i}=l}}^{k_{2}} q_{i}^{2} C_{i i}+2 \sum_{\substack{j, i=1 \\ i>j \\ q_{i}+q_{j}=l}}^{k_{2}} q_{i} q_{j} C_{i j}-2 \sum_{\substack{j, i=1 \\ i>j \\ q_{i}-q_{j}=l}}^{k_{2}} q_{i} q_{j} \overline{C_{i j}}=0
$$




$$
4 \sum_{\substack{i=1 \\ q_{i}=l}}^{k_{2}} q_{i} C_{i}+\sum_{\substack{i=1 \\ 2 q_{i}=l}}^{k_{2}} q_{i}^{2} E_{i i}-2 \sum_{\substack{j, i=1 \\ i>j \\ q_{i}+q_{j}=l}}^{k_{2}} q_{i} q_{j} E_{i j}+2 \sum_{\substack{j, i=1 \\ i>j \\ q_{i}-q_{j}=l}}^{k_{2}} q_{i} q_{j} \overline{E_{i j}}=0
$$

with

$$
\begin{array}{rr}
B_{i}=g\left(b_{0}, b_{i}\right), \quad A_{i}=g\left(b_{0}, a_{i}\right), & C_{i}=g\left(b_{0}, c_{i}\right), \quad D_{i}=g\left(b_{0}, d_{i}\right) \\
A_{i j}=g\left(a_{i}, a_{j}\right)+g\left(b_{i}, b_{j}\right) & \overline{A_{i j}}=g\left(a_{i}, a_{j}\right)-g\left(b_{i}, b_{j}\right) \\
D_{i j}=g\left(a_{i}, b_{j}\right)+g\left(a_{j}, b_{i}\right) & \overline{D_{i j}}=g\left(a_{i}, b_{j}\right)-g\left(a_{j}, b_{i}\right) \\
C_{i j}=g\left(c_{i}, c_{j}\right)+g\left(d_{i}, d_{j}\right) & \overline{C_{i j}}=g\left(c_{i}, c_{j}\right)-g\left(d_{i}, d_{j}\right) \\
E_{i j}=g\left(c_{i}, d_{j}\right)+g\left(c_{j}, d_{i}\right) & \overline{E_{i j}}=g\left(c_{i}, d_{j}\right)-g\left(c_{j}, d_{i}\right) .
\end{array}
$$

From the linear independence of the goniometric functions sin and cos, we get that

$$
\begin{aligned}
& g\left(a_{i}, c_{j}\right)=g\left(a_{i}, d_{j}\right)=0 \\
& g\left(b_{i}, c_{j}\right)=g\left(b_{i}, d_{j}\right)=0
\end{aligned}
$$

$\forall i \in\left\{1, . ., k_{1}\right\}, \quad \forall j \in\left\{1, . ., k_{2}\right\}$.

We first prove that $a_{t}=b_{t}=0, \quad \forall t \in\left\{1, \ldots, k_{1}\right\}$.

Let us look at the case that $k_{1}=1$, which means that $a_{1}$ and $b_{1}$ are not both zero. For $l=2 p_{1}$, we have from (5.3) and (5.4)

$$
\begin{gathered}
g\left(a_{1}, a_{1}\right)-g\left(b_{1}, b_{1}\right)=0 \\
g\left(a_{1}, b_{1}\right)=0 .
\end{gathered}
$$

The only solution is that $a_{1}=(a, a), \quad b_{1}=(b, b)$ with $a$ and $b$ not both equal to 0 . Taking $l=p_{1}$, we get from (5.3) that

$$
g\left(b_{0}, b_{1}\right)=0
$$

which means that $b_{0}=(c, c)$. From (5.7) and (5.8) it is easy to see that $\forall j \in\left\{1, \ldots, k_{2}\right\}$ : $g\left(c_{j}, c_{j}\right)=g\left(d_{j}, d_{j}\right)=0$ and this gives a contradiction with (5.2) the constant term in $g(\dot{\alpha}, \dot{\alpha})$.

We now prove, in a similar way, the general case $k_{1}>1$. Suppose that $\forall t \in\left\{1, \ldots, k_{1}\right\}, \quad a_{t}, b_{t}$ are noth both zero. Take $l=2 p_{k_{1}}$ then from (5.3) and (5.4) we have

$$
\begin{gathered}
g\left(a_{k_{1}}, a_{k_{1}}\right)-g\left(b_{k_{1}}, b_{k_{1}}\right)=0 \\
g\left(a_{k_{1}}, b_{k_{1}}\right)=0 .
\end{gathered}
$$

The only solution is that $a_{k_{1}}=\left(a_{k_{1} 1}, a_{k_{1} 1}\right)$ and $b_{k_{1}}=\lambda a_{k_{1}}, \quad \lambda \in \mathbb{R}$. If we take $l=p_{k_{1}}+p_{k_{1}-1}$ then from (5.3) and (5.4)

$$
g\left(a_{k_{1}}, a_{k_{1}-1}\right)-g\left(b_{k_{1}}, b_{k_{1}-1}\right)=0
$$




$$
g\left(a_{k_{1}}, b_{k_{1}-1}\right)+g\left(a_{k_{1}-1}, b_{k_{1}}\right)=0 .
$$

Substituting $b_{k_{1}}=\lambda a_{k_{1}}$ in this equations gives that $a_{k_{1}-1}$ and $b_{k_{1}-1}$ are of the same form as $a_{k_{1}}$ and $b_{k_{1}}$. It is easy to see that $\forall t, a_{t}$ and $b_{t}$ are of this form, such that $g\left(a_{t}, a_{t}\right)=g\left(b_{t}, b_{t}\right)=0$. Again from (5.7) and (5.8) follows that also $g\left(c_{t}, c_{t}\right)=g\left(d_{t}, d_{t}\right)=0$, which gives a contradiction with (5.2).

We obtain that $k_{1}$ must be equal to 0 .

In the next step we prove that $k_{2}$ can not be greater then 1 .

Suppose $k_{2}>1$ and $\forall t \in\left\{1, \ldots, k_{2}\right\}, \quad c_{t}$ and $d_{t}$ are not both equal to zero. Take $l=2 q_{k_{2}}$ in (5.5) and (5.6), then

$$
\begin{gathered}
g\left(c_{k_{2}}, c_{k_{2}}\right)+g\left(d_{k_{2}}, d_{k_{2}}\right)=0 \\
g\left(c_{k_{2}}, d_{k_{2}}\right)=0 .
\end{gathered}
$$

There are two cases.

Case 1. $g\left(c_{k_{2}}, c_{k_{2}}\right) \neq 0$

Then there exists one solution, $c_{k_{2}}=(u, v), \quad d_{k_{2}}=(v, u)$ with $|u| \neq|v|$. Working out equation (5.5) and (5.6) for $l=q_{k_{2}}+q_{k_{2}-1}$, we get that $c_{k_{2}-1}=$ $\left(u_{k_{2}-1}, v_{k_{2}-1}\right), \quad d_{k_{2}-1}=\left(v_{k_{2}-1}, u_{k_{2}-1}\right)$ with $\left|u_{k_{2}-1}\right| \neq\left|v_{k_{2}-1}\right|$. We can see that $\forall t: c_{t}=\left(u_{t}, v_{t}\right)$ and $d_{t}=\left(v_{t}, u_{t}\right)$ with $\left|u_{t}\right| \neq\left|v_{t}\right|$. Take $l=q_{k_{2}}-q_{1}$ in (5.5) and (5.6), then

$$
\begin{aligned}
& g\left(c_{k_{2}}, c_{1}\right)-g\left(d_{k_{2}}, d_{1}\right)=0 \\
& g\left(c_{k_{2}}, d_{1}\right)-g\left(c_{1}, d_{k_{2}}\right)=0 .
\end{aligned}
$$

This gives a contradiction, namely

(1) or $c_{1}=d_{1}=0$

(2) or $c_{k_{2}}=d_{k_{2}}=0$,

(3) or $\left|u_{1}\right|=\left|v_{1}\right|$,

(4) or $|u|=|v|$.

Case 2. $g\left(c_{k_{2}}, c_{k_{2}}\right)=0$

Then $c_{k_{2}}=(u, u), \quad d_{k_{2}}=(v, v)$, from (5.5) and (5.6) it is easy to prove that $\forall t: c_{t}=\left(u_{t}, u_{t}\right), \quad d_{t}=\left(v_{t}, v_{t}\right)$. From $l=q_{k_{2}}$ we derive that also $g\left(b_{0}, b_{0}\right)=0$ and this gives a contradiction with the causal character of $\alpha$.

The only cases left, are $k_{1}$ equal to 0 and $k_{2}$ equal to 0 or 1 . If $k_{1}$ and $k_{2}$ are both 0 , then $\alpha$ is a straight line. The case where $k_{1}=0$ and $k_{2}=1$ gives, from (5.2),(5.5) and (5.6) we have that

$$
\begin{gathered}
2 g\left(b_{0}, b_{0}\right)-q_{1}^{2} g\left(c_{1}, c_{1}\right)+q_{1}^{2} g\left(d_{1}, d_{1}\right)= \pm 2 \\
g\left(c_{1}, c_{1}\right)+g\left(d_{1}, d_{1}\right)=0 \\
g\left(c_{1}, d_{1}\right)=g\left(b_{0}, c_{1}\right)=g\left(b_{0}, d_{1}\right)=0 .
\end{gathered}
$$

The only solution is $c_{1}=(u, v), \quad d_{1}=(v, u)$ and $b_{0}=(0,0)$ with $|u| \neq|v|$ and $v^{2}-u^{2}= \pm \frac{1}{q_{1}^{2}}$. 
If $\alpha$ is a spacelike curve, take $v=\frac{1}{q_{1}^{2}}, \quad u=0$ and we get

$$
\alpha(s)=\frac{1}{q_{1}^{2}}\left(\sinh \left(q_{1} s\right), \cosh \left(q_{1} s\right)\right) .
$$

If $\alpha$ is a timelike curve, take $v=0, \quad u=\frac{1}{q_{1}^{2}}$ and we get

$$
\alpha(s)=\frac{1}{q_{1}^{2}}\left(\cosh \left(q_{1} s\right), \sinh \left(q_{1} s\right)\right) .
$$

This means that $\alpha$ is a part of an orthogonal hyperbola.

\subsection{Curves of Chen-type 2 in $\mathbb{E}_{1}^{3}$.}

Theorem 5.2. A planar 2-type curve,lying in an isotropic plane of $\mathbb{E}_{1}^{3}$, is a null 2-type spacelike curve.

Proof. We take as degenerate plane in $\mathbb{E}_{1}^{3}$, with metric $g=-d x_{1}^{2}+d x_{2}^{2}+d x_{3}^{2}$, the plane with equation $x_{1}=x_{2}$. If $\alpha(s)=a+b s+c \cos (p s)+d \sin (p s), a, b, c, d \in \mathbb{R}^{3}$, $p \in \mathbb{R}_{0}$ then $g(\dot{\alpha}, \dot{\alpha})= \pm 1$ leads to

$$
\begin{gathered}
g(c, d)=g(b, c)=g(b, d)=0 \\
g(c, c)-g(d, d)=0 \\
g(b, b)+p^{2} g(c, c)= \pm 1 .
\end{gathered}
$$

The only solution is $c=\left(c_{1}, c_{1}, 0\right), \quad d=\left(d_{1}, d_{1}, 0\right) \quad$ and $b=\left(b_{1}, b_{1}, b_{2}\right)$ with $c_{1}, d_{1}, b_{1} \in \mathbb{R}, c_{1}$ and $d_{1}$ not both equal to 0 and with $b_{2}= \pm 1$. Up to a translation, we obtain a spacelike curve

$$
\alpha(s)=(a \cos s+b \sin s+c s, a \cos s+b \sin s+c s, \pm s)
$$

with $a$ and $b$ not both 0 and $c \in \mathbb{R}_{0}$.

If $\alpha(s)=a+b s+c \cosh (p s)+d \sinh (p s), a, b, c, d \in \mathbb{R}^{3}, \quad p \in \mathbb{R}_{0}$ then $g(\dot{\alpha}, \dot{\alpha})= \pm 1$ leads to

$$
\begin{gathered}
g(c, d)=g(b, c)=g(b, d)=0 \\
g(c, c)+g(d, d)=0 \\
g(b, b)-p^{2} g(c, c)= \pm 1 .
\end{gathered}
$$

The only solution is $c=\left(c_{1}, c_{1}, 0\right), \quad d=\left(d_{1}, d_{1}, 0\right) \quad$ and $b=\left(b_{1}, b_{1}, b_{2}\right)$ with $c_{1}, d_{1}, b_{1} \in \mathbb{R}, c_{1}$ and $d_{1}$ not both equal to 0 and with $b_{2}= \pm 1$. Up to a translation, we obtain a spacelike curve

$$
\alpha(s)=(a \cosh s+b \sinh s+c s, a \cosh s+b \sinh s+c s, \pm s)
$$

with $c \neq 0$ and $a$ and $b$ not both 0 . 
If

$$
\alpha(s)=a_{0}+\sum_{i=1}^{2}\left(a_{i} \cosh \left(p_{i} s\right)+b_{i} \sinh \left(p_{i} s\right)\right)
$$

with $a_{i}, b_{i} \in \mathbb{R}^{3}, \quad 0<p_{1}<p_{2}$, then $g(\dot{\alpha}, \dot{\alpha})= \pm 1$, leads to two cases:

Case 1. $\quad p_{2} \neq 3 p_{1}$, then

$$
\begin{aligned}
& g\left(a_{1}, b_{1}\right)=g\left(a_{2}, b_{2}\right)=0 \\
& g\left(a_{1}, a_{1}\right)+g\left(b_{1}, b_{1}\right)=0 \\
& g\left(a_{2}, a_{2}\right)+g\left(b_{2}, b_{2}\right)=0
\end{aligned}
$$

and the solution is $a_{13}=a_{23}=b_{13}=b_{23}=0$, which gives a contradiction, because $\alpha$ is the intersection of 2 planes.

Case 2. $p_{2}=3 p_{1}, \quad$ then

$$
\begin{gathered}
g\left(a_{2}, b_{2}\right)=g\left(a_{2}, a_{2}\right)+g\left(b_{2}, b_{2}\right)=0 \\
g\left(a_{1}, a_{1}\right)+g\left(b_{1}, b_{1}\right)-6 g\left(a_{1}, a_{2}\right)+6 g\left(b_{1}, b_{2}\right)=0 \\
g\left(a_{1}, b_{1}\right)-3 g\left(a_{1}, b_{2}\right)+3 g\left(b_{1}, a_{2}\right)=0
\end{gathered}
$$

and the solution is $a_{13}=a_{23}=b_{13}=b_{23}=0$, which gives again a contradiction.

If

$$
\alpha(s)=a_{0}+\sum_{i=1}^{2}\left(a_{i} \cos \left(p_{i} s\right)+b_{i} \sin \left(p_{i} s\right)\right)
$$

or

$$
\alpha(s)=a_{0}+a_{1} \cos \left(p_{1} s\right)+b_{1} \sin \left(p_{1} s\right)+a_{2} \cosh \left(p_{2} s\right)+b_{2} \sinh \left(p_{2} s\right)
$$

with $a_{i}, b_{i} \in \mathbb{R}^{3}, \quad 0<p_{1}<p_{2}$, then we obtain after some computations also a contradiction.

We want to classify all curves of Chen-type 2 in $\mathbb{E}_{1}^{3}$, but first we look at the null 2-type curves[CS].

Theorem 5.3. Up to rigid motions of $\mathbb{E}_{1}^{3}$, a non-planar curve $\alpha$ in $\mathbb{E}_{1}^{3}$ is a null 2-type curve if and only if $\alpha$ is a part of one of the following curves :

$$
\begin{array}{ccc}
\alpha(s)=(a s, b \cos s, b \sin s), & a, b \in \mathbb{R}_{0} ; \quad|a| \neq|b| ; \\
\alpha(s)=(a \cosh s, a \sinh s, b s), & a, b \in \mathbb{R}_{0} ; \quad|a| \neq|b| ; \\
\alpha(s)=(a \sinh s, a \cosh s, b s), & a, b \in \mathbb{R}_{0} ; \quad|a| \neq|b| .
\end{array}
$$

Remarks. * All these curves are lying on a cilinder; (1) on a circular cilinder, (2) and (3) on a hyperbolic cilinder. Curve (2) is a spacelike curve; the causal character of curves (1) and (3) depends on the choice of the constants. 
Proof. If $\alpha$ is of null 2-type, then $\alpha$ can be written as :

(i)

$$
\alpha(s)=a+b s+c \cos (p s)+d \sin (p s),
$$

or

$$
\alpha(s)=a+b s+c \cosh (p s)+d \sinh (p s),
$$

$a, b, c, d \in \mathbb{R}^{3}, \quad p \in \mathbb{R}_{0}$.

Case i. $\quad g(\dot{\alpha}, \dot{\alpha})=k, \quad k \in \mathbb{R}_{0}$ leads to

$$
\begin{gathered}
g(c, d)=g(b, c)=g(b, d)=0 \\
g(c, c)-g(d, d)=0 .
\end{gathered}
$$

i.1. If $c$ is a null-vector, then $d=\lambda c, \lambda \in \mathbb{R}$ and $\alpha$ is a planar curve, which gives a contradiction.

i.2. If $c$ is not a null-vector, then the plane $\{c, d\}$ is spacelike. We can take $c=\left(0, c_{0}, 0\right)$ and $d=\left(0,0, c_{0}\right)$, then $b=\left(b_{0}, 0,0\right)$ with $\left|b_{0}\right| \neq\left|p c_{0}\right| ; \alpha(s)$ is of form (1).

Case ii. $\quad g(\dot{\alpha}, \dot{\alpha})=k, \quad k \in \mathbb{R}_{0}$ leads to

$$
\begin{gathered}
g(c, d)=g(b, c)=g(b, d)=0 \\
g(c, c)+g(d, d)=0 .
\end{gathered}
$$

ii.1. If $c$ is a null-vector, then $d=\lambda c, \lambda \in \mathbb{R}$ and $\alpha$ is a planar curve, which gives a contradiction.

ii.2. If $c$ is not a null-vector, then the plane $\{c, d\}$ is timelike.

If $c$ is timelike, we can take $c=\left(c_{0}, 0,0\right)$ and $d=\left(0, c_{0}, 0\right)$, then $b=\left(0,0, b_{0}\right)$ with $\left|b_{0}\right| \neq\left|p c_{0}\right| ; \alpha(s)$ is of form (2).

If $c$ is spacelike, we can take $c=\left(0, c_{0}, 0\right)$ and $d=\left(c_{0}, 0,0\right)$, then $b=\left(0,0, b_{0}\right)$ with $\left|b_{0}\right| \neq\left|p c_{0}\right| ; \alpha(s)$ is of form (3).

We now look at the curves of Chen-type 2 with 2 eigenvalues different from 0 .

Theorem 5.4. Up to rigid motions of $\mathbb{E}_{1}^{3}$, a non-planar curve $\alpha$ in $\mathbb{E}_{1}^{3}$ is a 2-type curve with both eigenvalues different from zero if and only if $\alpha$ is a part of one of the following curves :

$$
\begin{gathered}
\alpha(s)=(\rho \sin s, \epsilon \cos s+a \cos (3 s), \epsilon \sin s+a \sin (3 s)), \quad \rho^{2}-12 a \epsilon=0 ; \quad \rho, \epsilon, a \in \mathbb{R}_{0} ; \\
\alpha(s)=\left(a \cosh s+\lambda b \sinh s-4 c e^{(3 \lambda s)},-b \cosh s-\lambda a \sinh s+4 c e^{(3 \lambda s)}, 2 d e^{\lambda s}\right) ; \\
d^{2}-6(a-b) c=0, \quad a, b, c, d \in \mathbb{R}_{0}, \quad \lambda \in\{-1,1\} ; \\
\alpha(s)=\left(a e^{s}+b \cosh (3 s), a e^{s}+b \sinh (3 s), c e^{-s}\right) ; \\
c^{2}+6 a b=0, \quad a, b, c \in \mathbb{R}_{0} ;
\end{gathered}
$$


$\alpha(s)=(\epsilon \cosh s+a \cosh (3 s), \epsilon \sinh s+a \sinh (3 s), \rho \cosh s) ; \quad \rho^{2}+12 a \epsilon=0 ; \quad \rho, \epsilon, a \in \mathbb{R}_{0} ;$

$\alpha(s)=(\epsilon \cosh s+a \cosh (3 s), \epsilon \sinh s+a \sinh (3 s), \rho \sinh s) ; \quad \rho^{2}+12 a \epsilon=0 ; \quad \rho, \epsilon, a \in \mathbb{R}_{0} ;$

$$
\begin{gathered}
\alpha(s)=\left(a e^{s}+b \sinh (3 s), a e^{s}+b \cosh (3 s), c e^{-s}\right) \\
c^{2}-6 a b=0, \quad a, b, c \in \mathbb{R}_{0}
\end{gathered}
$$

$\alpha(s)=(\epsilon \sinh s+a \sinh (3 s), \epsilon \cosh s+a \cosh (3 s), \rho \cosh s) ; \quad \rho^{2}-12 a \epsilon=0 ; \quad \rho, \epsilon, a \in \mathbb{R}_{0} ;$

$\alpha(s)=(\epsilon \sinh s+a \sinh (3 s), \epsilon \cosh s+a \cosh (3 s), \rho \sinh s) ; \quad \rho^{2}-12 a \epsilon=0 ; \quad \rho, \epsilon, a \in \mathbb{R}_{0}$.

Remarks. * All these curves are lying on a quadric; (1) on an elipsoid and the others on a hyperboloid.

* Curves (1), (3), (4) and (5) are spacelike curves; curves (6), (7) and (8) are timelike curves. The causal character of the other curves depends on the choice of the constants.

Proof. If $\alpha$ is of 2 -type with both eigenvalues different from 0 , then $\alpha$ can be written as :

$$
\alpha(s)=a_{0}+a \cos (p s)+b \sin (p s)+c \cos (q s)+d \sin (q s)
$$

$a, b, c, d \in \mathbb{R}^{3}, \quad 0<p<q$.

Case i. $\quad \alpha(s)=a_{0}+a \cos (p s)+b \sin (p s)+c \cos (q s)+d \sin (q s)$

$\overline{g(\dot{\alpha}, \dot{\alpha})}= \pm 1$ leads to

i.1. $q \neq 3 p$.

$$
\begin{gathered}
g(c, d)=g(b, c)=g(b, d)=g(a, d)=g(a, b)=g(a, c)=0 \\
g(c, c)-g(d, d)=0 \\
g(a, a)-g(b, b)=0 \\
p^{2}[g(a, a)+g(b, b)]+q^{2}[g(c, c)+g(d, d)]= \pm 2
\end{gathered}
$$

It is impossible that one of these vectors is timelike (different from zero), and they can't be all spacelike, so there must be at least one, say $c$, with $g(c, c)=0$. $c$ is perpendicular to the spacelike plane $\{a, b\}$, so $c=0$, but the same is true for $d$, thus $d=0$ which gives a contradiction.

i.2. $q=3 p$.

$$
\begin{gathered}
g(c, c)-g(d, d)=g(c, d)=0 \\
g(b, d)-g(a, c)=g(a, d)+g(b, c)=0
\end{gathered}
$$




$$
\begin{gathered}
g(b, b)-g(a, a)+6[g(a, c)+g(b, d)]=0 \\
g(a, b)-3[g(a, d)-g(b, c)]=0 \\
p^{2}[g(a, a)+g(b, b)+9 g(c, c)+9 g(d, d)]= \pm 2
\end{gathered}
$$

i.2.1. If $c$ is a null-vector, then $d=\lambda c, \lambda \in \mathbb{R}$. From (2) follows that $\left(1+\lambda^{2}\right) g(a, c)=0$ and thus $g(a, c)=g(b, d)=0$. Using (3) and (4) we get that

$$
\begin{gathered}
g(b, b)-g(a, a)=0 \\
g(a, b)=0,
\end{gathered}
$$

we have the same conditions as in case i.1, which gives us again a contradiction.

i.2.2. If $c=0$, then $g(d, d)=0$ and $d$ is perpendicular to the spacelike plane $\{a, b\}$, so $d=0$ which gives the contradiction.

i.2.3. If $g(c, c) \neq 0$, then the plane $\{c, d\}$ is spacelike. Take $c=\left(0, c_{0}, 0\right), d=\left(0,0, c_{0}\right)$, from (2) follows that if $a=\left(a_{1}, a_{2}, a_{3}\right)$, then $b=\left(b_{1},-a_{3}, a_{2}\right)$. We have that

$$
\begin{gathered}
\alpha(s)=\left(a_{1} \cos (p s)+b_{1} \sin (p s), a_{2} \cos (p s)-a_{3} \sin (p s)+c_{0} \cos (3 p s),\right. \\
\left.a_{3} \cos (p s)+a_{2} \sin (p s)+c_{0} \sin (3 p s)\right) .
\end{gathered}
$$

Let

$$
\begin{array}{ll}
a_{2}=\epsilon \cos (\theta) & a_{1}=\rho \sin (\phi) \\
a_{3}=\epsilon \sin (\theta) & b_{1}=\rho \cos (\phi)
\end{array}
$$

then $\alpha(s)$ becomes

$$
\alpha(s)=\left(\rho \sin (p s+\varphi), \epsilon \cos (p s+\theta)+c_{0} \cos (3 p s), \epsilon \sin (p s+\theta)+c_{0} \sin (3 p s)\right) .
$$

From the conditions (3) and (4) we deduce that

$$
\begin{gathered}
\theta+2 \varphi=0 \\
\rho^{2}-12 \epsilon c_{0}=0 .
\end{gathered}
$$

Transforming $\alpha(s)$ with the isometry

$$
\left(\begin{array}{ccc}
1 & 0 & 0 \\
0 & \cos \beta & \sin \beta \\
0 & -\sin \beta & \cos \beta
\end{array}\right)
$$

$\beta=-3 \varphi$ gives that

$$
\alpha(s)=(\rho \sin (s), \epsilon \cos (s)+a \cos (3 s), \epsilon \sin (s)+a \sin (3 s)),
$$

$\rho^{2}-12 a \epsilon=0, \quad 3 a-\epsilon= \pm 1, \quad \rho, \epsilon, a \in \mathbb{R}_{0}$. Which means that $\alpha$ is of form (1).

Case ii. $\quad \alpha(s)=a_{0}+a \cosh (p s)+b \sinh (p s)+c \cosh (q s)+d \sinh (q s)$ $g(\dot{\alpha}, \dot{\alpha})= \pm 1$ leads to 
ii.1. $q \neq 3 p$.

$$
\begin{gathered}
g(c, d)=g(b, c)=g(b, d)=g(a, d)=g(a, b)=g(a, c)=0 \\
g(c, c)+g(d, d)=0 \\
g(a, a)+g(b, b)=0 \\
p^{2}[g(b, b)-g(a, a)]+q^{2}[g(d, d)-g(c, c)]= \pm 2
\end{gathered}
$$

If all these vectors are different from zero and not null vectors, then there would be two perpendicular, timelike vectors in $\mathbb{E}_{1}^{3}$, which is impossible. So there must be at least one vector,say $c$, with $g(c, c)=0 . c$ is perpendicular to the timelike plane $\{a, b\}$, so $c=0$, but the same is true for $d$, thus $d=0$ which gives a contradiction.

ii.2. $q=3 p$.

$$
\begin{gathered}
g(c, c)+g(d, d)=g(c, d)=0 \\
g(b, d)+g(a, c)=g(a, d)+g(b, c)=0 \\
g(b, b)+g(a, a)+6[g(b, d)-g(a, c)]=0 \\
g(a, b)-3[g(a, d)-g(b, c)]=0 \\
p^{2}[g(b, b)-g(a, a)-9 g(c, c)+9 g(d, d)]= \pm 2
\end{gathered}
$$

ii.2.1. If $c$ is a null-vector, then $d=\lambda c, \lambda \in \mathbb{R}$. From (2) follows that

$$
\left(1-\lambda^{2}\right) g(b, c)=0
$$

If $g(b, c)=0$, then also $g(a, c)=0$. Using (3) and (4) we get that

$$
\begin{gathered}
g(b, b)+g(a, a)=0 \\
g(a, b)=0,
\end{gathered}
$$

we have the same conditions as ii.1, which gives us again a contradiction.

If $\lambda^{2}=1$, then we get that

$$
\begin{gathered}
g(a+\lambda b, c)=0 \\
g(a+\lambda b, a+\lambda b)-12 g(a-\lambda b, c)=0 \\
g(a-\lambda b, a-\lambda b)=0 .
\end{gathered}
$$

It is easy to verify that $c$ and $a-\lambda b$ are two linear independent null vectors, so the plane $\{c, a-\lambda b\}$ is timelike. Take $a-\lambda b=\left(a_{0}, a_{0}, 0\right), \quad a_{0} \neq 0$ and $c=\left(-c_{0}, c_{0}, 0\right), \quad c_{0} \neq 0$. Calculating $a+\lambda b$, we get from the conditions above that $a+\lambda b=\left(b_{0},-b_{0}, b_{1}\right)$ with $b_{1}^{2}-24 a_{0} c_{0}=0$. Deducing $a$ and $b$ from $a-\lambda b, a+\lambda b$, we get a curve $\alpha$ of form ( 2 ).

ii.2.2. If $c=0$, then $g(d, d)=0$ and $d$ is perpendicular to the timelike plane $\{a, b\}$, so $d=0$ which gives the contradiction.

ii.2.3. If $g(c, c) \neq 0$, then the plane $\{c, d\}$ is timelike. 
If $c$ is timelike, take $c=\left(c_{0}, 0,0\right), \quad d=\left(0, c_{0}, 0\right)$. From (2) follows that if $a=\left(a_{1}, a_{2}, a_{3}\right)$, then $b=\left(a_{2}, a_{1}, b_{3}\right)$. We have that

$\alpha(s)=\left(a_{1} \cosh (p s)+a_{2} \sinh (p s)+c_{0} \cosh (3 p s), a_{2} \cosh (p s)+a_{1} \sinh (p s)+c_{0} \cosh (3 p s)\right.$,

$$
\left.a_{3} \cosh (p s)+b_{3} \sinh (p s)\right) \text {, }
$$

with

$$
\left(a_{3}+b_{3}\right)^{2}+12 c_{0}\left(a_{1}-a_{2}\right)=0
$$

and

$$
\left(a_{3}-b_{3}\right)^{2}+12 c_{0}\left(a_{1}+a_{2}\right)=0
$$

If $b_{3}= \pm a_{3}$ then $a_{1}=\mp a_{2}$ and $a_{3}^{2}+6 a_{1} c_{0}=0$, which means that $\alpha$ is of form (3). If $a_{3}^{2}>b_{3}^{2}$, let then

$$
\begin{array}{ll}
a_{1}=\epsilon \cosh (\theta) & a_{3}=\rho \cosh (\phi) \\
a_{2}=\epsilon \sinh (\theta) & b_{3}=\rho \sinh (\phi)
\end{array}
$$

then $\alpha(s)$ becomes

$$
\alpha(s)=\left(\epsilon \cosh (p s+\theta)+c_{0} \cosh (3 p s), \epsilon \sinh (p s+\theta)+c_{0} \sin (3 p s), \rho \cosh (p s+\varphi)\right) .
$$

From the conditions (3) and (4) we deduce that

$$
\begin{gathered}
\theta+2 \varphi=0 \\
\rho^{2}+12 \epsilon c_{0}=0 .
\end{gathered}
$$

After some transformations $\alpha$ is of form (4).

If $a_{3}^{2}<b_{3}^{2}$, we get by similar computations that $\alpha$ takes form (5).

All the same can be done when $c$ is spacelike, this gives us forms (6), (7) and (8).

Case iii. $\quad \alpha(s)=a_{0}+a \cos (p s)+b \sin (p s)+c \cosh (q s)+d \sinh (q s)$ $g(\dot{\alpha}, \dot{\alpha})= \pm 1$ leads to

$$
\begin{aligned}
& g(c, d)=g(b, c)= g(b, d)=g(a, d)=g(a, b)=g(a, c)=0 \\
& g(c, c)+g(d, d)=0 \\
& g(a, a)-g(b, b)=0
\end{aligned}
$$

If all these vectors are different from zero and not null vectors, then there would be three,orthogonal spacelike vectors in $\mathbb{E}_{1}^{3}$, which is impossible. So there must be at least one vector, say $c$, with $g(c, c)=0 . c$ is perpendicular to the spacelike plane $\{a, b\}$, so $c=0$, but the same is true for $d$, thus $d=0$ which gives a contradiction. 
CHAPTER II

\section{SURFACES IN MINKOWSKI SPACE}

\section{$\S 1$. INTRODUCTION}

A semi-Riemannian surface $M$ is a semi-Riemannian manifold of dimension 2 in the n-dimensional Minkowski space $\mathbb{E}_{1}^{n}$, i.e. the space $\mathbb{R}^{n}$ with a Lorentzian metric $g=\sum_{i=1}^{n} \varepsilon_{i} d x_{i}^{2}$, with all $\varepsilon_{i}=1$, except one which is equal to -1 . For a coordinate system $u, v$ in $M$ the components of the metric tensor are traditionally denoted by

$$
E=g_{11}=g\left(\partial_{u}, \partial_{u}\right), \quad F=g_{12}=g_{21}=g\left(\partial_{u}, \partial_{v}\right), \quad G=g_{22}=g\left(\partial_{v}, \partial_{v}\right) .
$$

For a semi-Riemannian surface $M$ the metric induced on $M$, which we also denote by $g$, is non-degenerate, this means that

$$
E G-F^{2} \neq 0
$$

We also introduce the functions $g^{i j}$ which satisfy

$$
\sum_{k=1}^{2} g_{i k} g^{k j}=\delta_{i}^{j}
$$

where $\delta_{i}^{j}=0$ if $i \neq j$ and $\delta_{i}^{i}=1$.

The Laplacian of $M$ is defined by

$$
\Delta=\frac{1}{\sqrt{|g|}}\left[\frac{\partial}{\partial u}\left(\sqrt{|g|}\left(g^{11} \frac{\partial}{\partial u}+g^{12} \frac{\partial}{\partial v}\right)\right)+\frac{\partial}{\partial v}\left(\sqrt{|g|}\left(g^{21} \frac{\partial}{\partial u}+g^{22} \frac{\partial}{\partial v}\right)\right)\right]
$$

where $|g|=\left|\operatorname{det}\left(g_{i j}\right)\right|$.

We repeat some wellknown formulas.

The standard connection $D$ on $\mathbb{E}_{1}^{n}$ induces the Levi-Civita-connection $\nabla$ on $M$, and we have :

$$
\begin{aligned}
& D_{X}^{Y}=\nabla_{X} Y+h(X, Y) \\
& D_{X} \xi=-A_{\xi} X+D_{X}^{\perp} \xi,
\end{aligned}
$$


with $X, Y \in T M, \xi$ a unit normal vector field on $M$, and where $h$ is the second fundamental form of $M$. $A_{\xi}$ is the shape operator of $M$ with respect to $\xi$ and $D^{\perp}$ the normal connection on $M$. (1.1) is known as the Gauss formula and (1.2) as the Weingarten formula.

The mean curvature vector field $H$ of $M$ is defined by

$$
H=\frac{1}{2}\left(\varepsilon_{1} h\left(e_{1}, e_{1}\right)+\varepsilon_{2} h\left(e_{2}, e_{2}\right)\right)
$$

with $\left(e_{1}, e_{2}\right)$ an orthonormal bases of $T M$ and $\varepsilon_{i}=g\left(e_{i}, e_{i}\right)= \pm 1$.

Equivalently,

$$
H=\frac{1}{2} \sum_{i=3}^{n} \varepsilon_{i} \cdot \operatorname{tr} A_{\xi_{i}} \cdot \xi_{i}
$$

with $\left\{\xi_{i}\right\}$ an orthonormal bases of $T M^{\perp}$ and $\varepsilon_{i}=g\left(\xi_{i}, \xi_{i}\right)= \pm 1$.

We'll need the notion of a surface of translation. By a surface of translation $M$ of $\mathbb{E}_{1}^{n}$, we mean a surface given by an immersion

$$
X: U \subset \mathbb{E}^{2} \rightarrow \mathbb{E}_{1}^{n}:(x, y) \rightarrow\left(x, y, f_{3}(x)+g_{3}(y), \ldots, f_{n}(x)+g_{n}(y)\right) .
$$

The best known surface of translation in the 3-dimensional Euclidean space $\mathbb{E}^{3}$ is the surface of Scherk (Figure 8, p.99). This is the surface $M$ defined by

$$
e^{z} \cos x=\cos y
$$

When $\cos x \cos y>0$, we can solve explicitly for $z$ :

$$
z=\log \left(\frac{\cos y}{\cos x}\right)
$$

The ruled surfaces are another well known class of surfaces. By a ruled surface $M$ of $\mathbb{E}_{1}^{n}$, we mean a surface which can be parameterized as

$$
X(s, t)=\alpha(s)+t \beta(s),
$$

where $\alpha$ and $\beta$ are two curves of $\mathbb{E}_{1}^{n}$. For fixed $s$, we obtain straight lines through $\alpha(s)$; these straight lines are called the rules (or rulings) of $M$. The ruled surfaces which are not generalized cones or cylinders, or tangent developables, are called scrolls. A generalized cone of $\mathbb{E}_{1}^{n}$ is parameterized by

$$
X(s, t)=V+t[c(s)-V] \text {, }
$$

where $V \in \mathbb{R}^{n}$ and $c(s)$ is an immersed curve in $\mathbb{E}_{1}^{n}$. A tangent developable surface consists of the tangents to a curve $\alpha$. It can therefore be parameterized by

$$
X(s, t)=\alpha(s)+t \dot{\alpha}(s)
$$

We give some wellknown examples of ruled surfaces in $\mathbb{E}^{3}$. 
Our first example is the "standard" Möbius strip, which can be parameterized by

$$
X(s, t)=(\cos s, \sin s, 0)+t(\cos (s / 2) \cos s, \cos (s / 2) \sin s, \sin (s / 2)),
$$

with $|t|<\frac{1}{2}$. (Figure 1, p.91)

The second example is the right helicoid, parameterized by

$$
X(s, t)=(0,0, b s)+t(\cos s, \sin s, 0), \quad b \neq 0 .
$$

This surface is generated by a line which moves along the z-axis in such a way that it remains parallel to the (x,y)-plane, and passes through the points of a circular helix. The lines $t=$ constant are helices. (Figure 4, p.95)

The third example is a quadric surface, namely the elliptic hyperboloid of one sheet. This surface has the equation (Figure 2, p.92)

$$
\frac{x^{2}}{a^{2}}+\frac{y^{2}}{b^{2}}-\frac{z^{2}}{c^{2}}=1 \text {. }
$$

It is doubly ruled and may be parameterized as

$$
X(s, t)=(a \cos (s), b \sin s, 0)+t(a \sin s,-b \cos s, c) .
$$

Planes perpendicular to the $\mathrm{z}$-axis intersect the surface in similar ellipses, while planes perpendicular to the other axes intersect it in hyperbolas. When $a=b$, it may be obtained by revolving a hyperbola around the $z$-axis (hyperboloid of revolution).

The last example is also a quadric surface, namely the hyperbolic paraboloid. This surface has the equation

$$
z=\frac{x^{2}}{a^{2}}-\frac{y^{2}}{b^{2}}
$$

It is also a doubly ruled surface and it can be parameterized by

$$
X(s, t)=\left(a s, 0, s^{2}\right)+t(a, b, 2 s) .
$$

Planes perpendicular to the $y$-axis intersect the surface in parabolas, and planes perpendicular to the $\mathrm{x}$-axis intersect the surface in parabolas pointing in the other way. Planes perpendicular to the $z$-axis intersect the surface in hyperboles pointing in one direction when the plane lies above the $(x, y)$-plane, and in the other direction when the plane lies below the $(x, y)$ plane; the $(x, y)$-plane itself intersects the surface in two intersecting straight lines. (Figures 3a, 3b, p. 93,94) 


\section{§2. MINIMAL SURFACES}

\subsection{Minimal surfaces in $\mathbb{E}_{1}^{3}$.}

The study of minimal surfaces started with the more intuitive meaning of minimal surfaces, namely surfaces of least area among a family of surfaces having the same boundary. Lagrange defined in 1760 the minimal surfaces as surfaces whose mean curvature vanishes. A surface $M$ in $\mathbb{E}_{1}^{n}$ is called minimal if and only if the mean curvature vector field is equal to zero, so

$$
H=0
$$

or equivalently, if

$$
\operatorname{tr} A_{\xi_{i}}=0, \text { for } i=3, \ldots, n \text {; }
$$

with $\left\{\xi_{i}\right\}$ an orthonormal bases of $T M^{\perp}$ and $A_{\xi_{i}}$ the shape operator of $M$ with respect to $\xi_{i}$. This condition is precisely the one which must be satisfied by a surface which is a critical point for the area function among all surfaces with the same boundary curve.[VdW1]

Historically, the three first examples of non-trivial minimal surfaces in 3-dimensional Euclidean space $\mathbb{E}^{3}$ were the catenoids, the helicoids and the minimal translation surfaces. The catenoids are ( besides the planes ) the only minimal surfaces of revolution (Euler 1744, Meusnier 1776). These surfaces are obtained by revolving a catenary $(x=a \cosh (z / a))$ around the z-axis. They can be parameterized by

$$
X(s, t)=\left(\sqrt{1+s^{2}} \cos t, \sqrt{1+s^{2}} \sin t, \sinh ^{-1}(s)\right) .
$$

The helicoids are (besides the planes) the only minimal ruled surfaces (Meusnier 1776, Catalan 1844). The minimal translation surfaces were discovered by Scherk in 1835; he proved that (besides the planes) essentially the surfaces with equation

$$
z=\log \left|\frac{\cos y}{\cos x}\right|
$$

are the only minimal surfaces of translation. Of all other minimal surfaces, Enneper's minimal surface is the best known. [see Sp]

The minimal surfaces in the 3 -dimensional Minkowski space $\mathbb{E}_{1}^{3}$ with metric $g=$ $d x_{1}^{2}+d x_{2}^{2}-d x_{3}^{2}$ were studied by Kobayashi in 1983 . He classified all the spacelike minimal - he called them "maximal" because the second variation of volume is allways negative definite for spacelike surfaces in $\mathbb{E}_{1}^{3}$ - rotation surfaces and ruled surfaces. Van de Woestyne classified in 1989 all the timelike minimal rotation surfaces and ruled surfaces and also all the minimal translation surfaces in $\mathbb{E}_{1}^{3}$ with metric $g=d x_{1}^{2}+d x_{2}^{2}-d x_{3}^{2}$.

We mention some of the results without proves. For the proves see [Ko] and [VdW1]. 
Theorem 2.1. Every minimal, spacelike surface of translation in $\mathbb{E}_{1}^{3}$ with metric $g=d x_{1}^{2}+d x_{2}^{2}-d x_{3}^{2}$, is congruent to a part of one of the following surfaces :

i. a spacelike plane,

ii. the surface of Scherk of the first kind,

$$
z(x, y)=\frac{1}{a} \log \left|\frac{\cosh (a y)}{\cosh (a x)}\right|
$$

with $a \in \mathbb{R}_{0}$ and $\tanh ^{2}(a x)+\tanh ^{2}(a y)<1$.

Theorem 2.2. Every minimal, timelike surface of translation in $\mathbb{E}_{1}^{3}$ with metric $g=d x_{1}^{2}+d x_{2}^{2}-d x_{3}^{2}$, is congruent to a part of one of the following surfaces:

i. the timelike plane,

ii. the surface of Scherk of the first kind,

$$
z(x, y)=\frac{1}{a} \log \left|\frac{\cosh (a y)}{\cosh (a x)}\right|
$$

with $a \in \mathbb{R}_{0}$ and $\tanh ^{2}(a x)+\tanh ^{2}(a y)>1$,

iii. the surface of Scherk of the second kind,(Figure 9, p. 100)

$$
z(x, y)=\frac{1}{a} \log \left|\frac{\cosh (a y)}{\sinh (a x)}\right|
$$

with $a \in \mathbb{R}_{0}$,

iv. the surface of Scherk of the third kind,(Figure 10, p. 101)

$$
z(x, y)=\frac{1}{a} \log \left|\frac{\sinh (a y)}{\sinh (a x)}\right|
$$

with $a \in \mathbb{R}_{0}$,

v. a flat B-scroll over a null-curve,(Figure 13, p.104)

$$
z(x, y)= \pm x+g(y)
$$

and $g(y)$ is an arbitrary function.

Theorem 2.3. Every minimal, spacelike ruled surface in $\mathbb{E}_{1}^{3}$ is congruent to a part of one of the following surfaces:

(1) a spacelike plane;

(2) the helicoid of first kind;(Figure 4, p. 95)

$$
X(s, t)=\left(\cos (\sqrt{K} t)\left(-s+\frac{\kappa}{K}\right), \sin (\sqrt{K} t)\left(-s+\frac{\kappa}{K}\right), \frac{\tau t}{\sqrt{K}}\right)
$$

with $K=\kappa^{2}-\tau^{2}, \quad s<\min \left(\frac{-1}{\tau+\kappa}, \frac{1}{\tau-\kappa}\right)$ or $s>\max \left(\frac{-1}{\tau+\kappa}, \frac{1}{\tau-\kappa}\right)$, where $\kappa$ is the curvature and $\tau$ is the torsion of $\alpha$ with $|\kappa|>|\tau|>0$; 
(3) the helicoid of second kind;(Figure 5, p. 96)

$$
X(s, t)=\left(\cosh (\sqrt{K} t)\left(-s-\frac{\kappa}{K}\right), \frac{\tau t}{\sqrt{K}}, \sinh (\sqrt{K} t)\left(-s-\frac{\kappa}{K}\right)\right)
$$

with $K=\tau^{2}-\kappa^{2}, \quad s>\min \left(\frac{-1}{\tau+\kappa}, \frac{1}{\tau-\kappa}\right)$ or $s<\max \left(\frac{-1}{\tau+\kappa}, \frac{1}{\tau-\kappa}\right)$, where $\kappa$ is the curvature and $\tau$ is the torsion of $\alpha$ with $|\tau|>|\kappa|>0$;

(4) the conjugate of Enneper's surface of second kind;(Figure 7, p. 98)

$$
X(s, t)=\left(\frac{\kappa t^{2}}{2}+s, \frac{-\tau^{2} t^{3}}{6}+t-\kappa s t, \frac{\kappa \tau t^{3}}{6}+\tau s t\right),
$$

where $\kappa$ is the curvature and $\tau$ is the torsion of $\alpha$ with $|\tau|=|\kappa|>0$. $s<\frac{1}{2 \kappa}$ if $\kappa>0, \quad s>\frac{1}{2 \kappa}$ if $\kappa<0$, because $M$ is spacelike.

Theorem 2.4. Every minimal, timelike ruled surface in $\mathbb{E}_{1}^{3}$ is congruent to a part of one of the following surfaces:

(1) a timelike plane;

(2) the helicoid of first kind;(Figure 4, p. 95)

$$
X(s, t)=\left(\cos (\sqrt{K} t)\left(-s+\frac{\kappa}{K}\right), \sin (\sqrt{K} t)\left(-s+\frac{\kappa}{K}\right), \frac{\tau t}{\sqrt{K}}\right)
$$

with $K=\tau^{2}-\kappa^{2}, \quad \min \left(\frac{-1}{\tau+\kappa}, \frac{1}{\tau-\kappa}\right)<s<\max \left(\frac{-1}{\tau+\kappa}, \frac{1}{\tau-\kappa}\right)$, where $\kappa$ is the curvature and $\tau$ is the torsion of $\alpha$ with $|\tau|>|\kappa|>0$;

(3) the helicoid of second kind;(Figure 5, p. 96)

$$
X(s, t)=\left(-\cosh (\sqrt{K} t)\left(s+\frac{\kappa}{K}\right), \frac{\tau t}{\sqrt{K}},-\sinh (\sqrt{K} t)\left(s+\frac{\kappa}{K}\right)\right)
$$

with $K=\kappa^{2}-\tau^{2}, \quad s<\min \left(\frac{-1}{\tau+\kappa}, \frac{1}{\tau-\kappa}\right)$ or $s>\max \left(\frac{-1}{\tau+\kappa}, \frac{1}{\tau-\kappa}\right)$, where $\kappa$ is the curvature and $\tau$ is the torsion of $\alpha$ with $|\kappa|>|\tau|>0$;

(4) the helicoid of third kind;(Figure 6, p. 97)

$$
X(s, t)=\left(-\sinh (\sqrt{K} t)\left(s+\frac{\kappa}{K}\right), \frac{\tau t}{\sqrt{K}},-\cosh (\sqrt{K} t)\left(s+\frac{\kappa}{K}\right)\right)
$$

with $K=\kappa^{2}+\tau^{2}$, where $\kappa$ is the curvature and $\tau$ is the torsion of $\alpha$;

(5) the conjugate of Enneper's surface of second kind;(Figure 7, p. 98)

$$
X(s, t)=\left(\frac{\kappa t^{2}}{2}+s, \frac{-\kappa \tau t^{3}}{6}-\tau s t, \frac{\tau^{2} t^{3}}{6}+t+\kappa s t\right),
$$

where $\kappa$ is the curvature and $\tau$ is the torsion of $\alpha$ with $|\tau|=|\kappa|>0$. $s>\frac{-1}{2 \kappa}$ if $\kappa>0, \quad s<\frac{-1}{2 \kappa}$ if $\kappa<0$, because $M$ is timelike;

(6) a flat B-scroll over a null-curve (Figure 13, p.104). 


\subsection{Minimal translation surfaces.}

Like we saw in the previous paragraph, minimal surfaces of translation in the 3dimensional Minkowski space $\mathbb{E}_{1}^{3}$ with metric $g=d x_{1}^{2}+d x_{2}^{2}-d x_{3}^{2}$, are classified by $\mathrm{I}$. Van de Woestyne ([VdW1],[VdW2]). We'll classify the minimal surfaces of translation in the n-dimensional Minkowski space $\mathbb{E}_{1}^{n}$.

We now assume that $\mathbb{E}_{1}^{n}$ has the following Lorentzian metric, $g=-d x_{1}^{2}+\sum_{i=2}^{n} d x_{i}^{2}$. Let $M$ be a surface of translation of $\mathbb{E}_{1}^{n}$ given by an immersion $X$,

$$
X: U \subset \mathbb{E}^{2} \rightarrow \mathbb{E}_{1}^{n}:(x, y) \rightarrow\left(x, y, f_{3}(x)+g_{3}(y), \ldots, f_{n}(x)+g_{n}(y)\right) .
$$

A bases for the tangent plane $T M$ is given by

$$
\begin{gathered}
\frac{\partial X}{\partial x}=\left(1,0, \frac{d f_{3}(x)}{d x}, \ldots, \frac{d f_{n}(x)}{d x}\right) \\
\frac{\partial X}{\partial y}=\left(0,1, \frac{d g_{3}(y)}{d y}, \ldots, \frac{d g_{n}(y)}{d y}\right) .
\end{gathered}
$$

The normal space $T^{\perp} M$ is spanned by

$$
\xi_{i}=\left(\frac{d f_{i}(x)}{d x}, \frac{d g_{i}(y)}{d y}, 0, \ldots, 1, \ldots, 0\right), \text { for } i=3, \ldots, n
$$

with the $i$-th component equal to 1 .

From the condition of minimality,

$$
\operatorname{tr} A_{\xi_{i}}=0, \text { for } i=3, \ldots, n \text {; }
$$

we obtain the following system of differential equations

$$
\left(1+\sum_{j=3}^{n} \dot{g}_{j}^{2}(y)\right) \ddot{f}_{i}(x)+\left(-1+\sum_{j=3}^{n} \dot{f}_{j}^{2}(x)\right) \ddot{g}_{i}(y)=0
$$

for $i=3, \ldots, n$.

Case 1. If

$$
\sum_{j=3}^{n} \dot{f}_{j}^{2}=1
$$

then the condition of minimality implies

$$
\ddot{f}_{i}(x)=0, \quad \forall i \in\{3, \ldots, n\}
$$

such that

$$
f_{i}(x)=a_{i} x+b_{i}
$$


with $\forall i a_{i}, b_{i} \in \mathbb{R}$ and $\sum_{j=3}^{n} a_{j}^{2}=1 . g_{i}(y)$ is an arbitrary function and we get a generalized B-scroll,

$$
F(x, y)=x\left(1,0, a_{3}, . ., a_{n}\right)+\left(0, y, g_{3}(y), . ., g_{n}(y)\right) \text {. }
$$

\section{Case 2. If}

$$
\sum_{j=3}^{n} \dot{f}_{j}^{2} \neq 1
$$

then

$$
\frac{\ddot{f}_{i}(x)}{-1+\sum_{j=3}^{n} \dot{f}_{j}^{2}(x)}=\frac{-\ddot{g}_{i}(y)}{1+\sum_{j=3}^{n} \dot{g}_{j}^{2}(y)}=c_{i},
$$

with $c_{i} \in \mathbb{R}, \quad \forall i=3, . ., n$.

Case 2.1. If $c_{i}=0, \forall i \in\{3, . ., n\}$, we get that $M$ is a plane.

Case 2.2. Suppose that $\exists i: c_{i} \neq 0$. Without loss of generality, we may assume that $c_{3} \neq 0$. We get that

$$
\frac{\ddot{f}_{i}(x)}{\ddot{f}_{3}}=\frac{c_{i}}{c_{3}}
$$

such that

$$
\dot{f}_{i}=\frac{c_{i}}{c_{3}} \dot{f}_{3}+k_{i}
$$

with $k_{i} \in \mathbb{R}, \quad \forall i \in\{3, . ., n\}$ and $k_{3}=0$.

Substituting $\dot{f}_{i}$ in

$$
\ddot{f_{3}}=c_{3}\left(-1+\sum_{j=3}^{n} \dot{f}_{j}^{2}\right)
$$

we get that

$$
c_{3} \ddot{f}_{3}=\dot{f}_{3}^{2}\left(c_{3}^{2}+\cdots+c_{n}^{2}\right)+2 c_{3} \dot{f}_{3}\left(c_{4} k_{4}+\cdots+c_{n} k_{n}\right)+c_{3}^{2}\left(-1+k_{4}^{2}+\cdots+k_{n}^{2}\right) .
$$

This equation is equivalent with the following differential equation,

$$
\frac{c_{3} d y}{c_{3}^{2} K+2 c_{3} B y+A y^{2}}=d x
$$

with

$$
y=\dot{f_{3}}, \quad A=\sum_{j=3}^{n} c_{j}^{2}, \quad B=\sum_{j=4}^{n} c_{j} k_{j}, \quad K=-1+\sum_{j=4}^{n} k_{j}^{2} .
$$

Let $D$ be the discriminant, $D=4 c_{3}^{2}\left(B^{2}-A K\right)$. The above differential equation can then be written as

$$
\frac{c_{3} d y}{A\left[\left(y+\frac{c_{3} B}{A}\right)^{2}-\frac{D}{4 A^{2}}\right]}=d x \text {. }
$$


The following cases appear.

Case 2.2.1.

If $D=0$, then

$$
\frac{d y}{\left(y+\frac{B c_{3}}{A}\right)^{2}}=\frac{A}{c_{3}} d x .
$$

After integration, we find that

$$
\dot{f}_{3}=y=\frac{c_{3}}{A x+c_{3} l}-\frac{B c_{3}}{A} .
$$

One more integration, gives us that

$$
f_{3}(x)=\frac{-c_{3}}{A} \log \left|A x+l c_{3}\right|-\frac{B c_{3}}{A} x+q,
$$

with $l$ and $q$ integration constants. Using the relation between $f_{i}$ and $f_{3}$ and after some translations and reparameterizations, we get that $\forall i \in\{3, . ., n\}$,

$$
f_{i}(x)=\frac{-c_{i}}{A} \log |A x|+\left(k_{i}-c_{i} \frac{B}{A}\right) x .
$$

\section{Case 2.2.2.}

If $D<0$, then

$$
\frac{c_{3} d y}{\frac{D}{4 A}\left[\left(\frac{2 A y}{\sqrt{-D}}+\frac{2 c_{3} B}{\sqrt{-D}}\right)^{2}+1\right]}=d x
$$

Integration gives

$$
\dot{f}_{3}=y=\frac{\sqrt{-D}}{2 A} \tan \left(\frac{\sqrt{-D}}{-2 c_{3}}(x+l)\right)-\frac{c_{3} B}{A} .
$$

After one more integration, we find that

$$
f_{3}(x)=\frac{-c_{3}}{A} \log \left|\cos \left(\sqrt{A K-B^{2}}(x+l)\right)\right|-\frac{B c_{3}}{A} x+q,
$$

with $l$ and $q$ integration constants. Using the relation between $f_{i}$ and $f_{3}$ and after some translations and reparameterizations, we get that $\forall i \in\{3, . ., n\}$,

$$
f_{i}(x)=\frac{-c_{i}}{A} \log \mid \cos \left(\sqrt{A K-B^{2}}(x+l) \mid+\left(k_{i}-c_{i} \frac{B}{A}\right) x .\right.
$$

Case 2.2.3. If $D>0$, then

$$
\frac{-c_{3} d y}{\frac{D}{4 A}\left[1-\left(\frac{2 A y}{\sqrt{D}}+\frac{2 c_{3} B}{\sqrt{D}}\right)^{2}\right]}=d x
$$


a. If $\left|\frac{2\left(A \dot{f}_{3}+B c_{3}\right)}{\sqrt{D}}\right|<1$, then after some integrations, we have that

$$
\dot{f}_{3}=y=\frac{\sqrt{D}}{2 A} \tanh \left(\frac{\sqrt{D}}{-2 c_{3}}(x+l)\right)-\frac{c_{3} B}{A},
$$

and thus

$$
f_{3}(x)=\frac{-c_{3}}{A} \log \left(\cosh \left(\sqrt{B^{2}-A K}(x+l)\right)\right)-\frac{B c_{3}}{A} x+q,
$$

with $l$ and $q$ integration constants. Using the relation between $f_{i}$ and $f_{3}$ and after some translations and reparameterizations, we get that $\forall i \in\{3, . ., n\}$,

$$
f_{i}(x)=\frac{-c_{i}}{A} \log \left(\cosh \left(\sqrt{B^{2}-A K} x\right)\right)+\left(k_{i}-c_{i} \frac{B}{A}\right) x .
$$

b. If $\left|\frac{2\left(A \dot{f}_{3}+B c_{3}\right)}{\sqrt{D}}\right|>1$, then after some integrations, we have that

$$
\dot{f}_{3}=y=\frac{\sqrt{D}}{2 A} \operatorname{coth}\left(\frac{\sqrt{D}}{-2 c_{3}}(x+l)\right)-\frac{c_{3} B}{A},
$$

and

$$
f_{3}(x)=\frac{-c_{3}}{A} \log \left|\sinh \left(\sqrt{B^{2}-A K}(x+l)\right)\right|-\frac{B c_{3}}{A} x+q,
$$

with $l$ and $q$ integration constants. Using the relation between $f_{i}$ and $f_{3}$ and after some translations and reparameterizations, we get that $\forall i \in\{3, . ., n\}$,

$$
f_{i}(x)=\frac{-c_{i}}{A} \log \left|\sinh \left(\sqrt{B^{2}-A K} x\right)\right|+\left(k_{i}-c_{i} \frac{B}{A}\right) x .
$$

We can do the same computations for $g_{i}(y)$, by replacing the constants $k_{i}$ by $l_{i}$. We get the following differential equation,

$$
\frac{c_{3} d z}{c_{3}^{2} L+2 c_{3} \tilde{B} z+A z^{2}}=d y
$$

with

$$
z=\dot{g}_{3}, \quad A=\sum_{j=3}^{n} c_{j}^{2}, \quad \tilde{B}=\sum_{j=4}^{n} c_{j} l_{j}, \quad L=1+\sum_{j=4}^{n} l_{j}^{2} .
$$

The discriminant, $\tilde{D}=4 c_{3}^{2}\left(\tilde{B}^{2}-A L\right)$, is allways negative which results in the only possible case that $\forall i \in\{3, . ., n\}$,

$$
g_{i}(y)=\frac{c_{i}}{A} \log \left|\cos \left(\sqrt{A L-\tilde{B}^{2}} y\right)\right|+\left(l_{i}-c_{i} \frac{\tilde{B}}{A}\right) y
$$

Summarizing, we get the following result. 
Theorem 2.5. $M$ is a minimal surface of translation in $\mathbb{E}_{1}^{n}$, with metric $g=-d x_{1}^{2}+\sum_{i=2}^{n} d x_{i}^{2}$, if and only if it is congruent to a part of one of the following surfaces,

(1) a plane;

(2) a generalized B-scroll;

(3) a surface parameterized by

$$
F(x, y)=\left(x, y, f_{3}(x)+g_{3}(y), \ldots, f_{n}(x)+g_{n}(y)\right),
$$

with $f_{i}$ and $g_{i}$ one of the previous forms.

For $n=3$, this becomes.

Theorem 2.6. $M$ is a minimal spacelike surface of translation in $\mathbb{E}_{1}^{3}$, with metric $g=-d x_{1}^{2}+d x_{2}^{2}+d x_{3}^{2}$, if and only if it is congruent to a part of one of the following surfaces,

(1) a spacelike plane;

(2) a surface parameterized by

$$
z(x, y)=\frac{1}{a} \log \left|\frac{\cos (a y)}{\sinh (a x)}\right|,
$$

with $a \in \mathbb{R}_{0}, \quad \operatorname{coth}^{2}(a x)-\tan ^{2}(a y)>1$. (Figure 11, p. 102)

Theorem 2.7. $M$ is a minimal timelike surface of translation in $\mathbb{E}_{1}^{3}$, with metric $g=-d x_{1}^{2}+d x_{2}^{2}+d x_{3}^{2}$, if and only if it is congruent to a part of one of the following surfaces,

(1) a timelike plane;

(2) a flat B-scroll;

$$
z(x, y)= \pm x+g(y)
$$

with $g(y)$ an arbitrary function;(Figure 13, p. 104)

(3) a surface parameterized by

$$
z(x, y)=\frac{1}{a} \log \left|\frac{\cos (a y)}{\cosh (a x)}\right|,
$$

with $a \in \mathbb{R}_{0} ;$ (Figure 12, p. 103)

(4) a surface parameterized by

$$
z(x, y)=\frac{1}{a} \log \left|\frac{\cos (a y)}{\sinh (a x)}\right|,
$$

with $a \in \mathbb{R}_{0}, \quad \operatorname{coth}^{2}(a x)-\tan ^{2}(a y)<1$. (Figure 11, p. 102) 


\section{§3. RULED SURFACES OF FINITE TYPE}

\subsection{Introduction.}

A classical result of Catalan states that the only ruled minimal surfaces in Euclidean 3 -space are the plane and the helicoid. Minimal submanifolds are contained in a much larger class of submanifolds, namely in the class of submanifolds of finite type.

In [CDVV] the ruled surfaces of finite type in a Euclidean space were studied, we recall some of the results.

Theorem 3.1. A ruled surface $M$ in $\mathbb{E}^{n}$ is of finite type if and only if $M$ is a cylinder over a curve of finite type or $M$ is a part of a helicoid in an affine subspace $\mathbb{E}^{3}$.

If we look at the 3-dimensional Euclidean space and consider that the only curves of finite type in $\mathbb{E}^{2}$ are lines and circles, we get the following theorem.

Theorem 3.2. A ruled surface $M$ in $\mathbb{E}^{3}$ is of finite type if and only if $M$ is a part of a plane, a circular cylinder or a helicoid.

In [D] all the ruled submanifolds of finite type in Euclidean space were classified.

Theorem 3.3. A ruled submanifold $M^{n+1}$ in $\mathbb{E}^{n+p}$ is of finite type if and only if $M^{n+1}$ is a part of a cylinder on a curve of finite type or a part of a generalized helicoid.

The minimal ruled surfaces in $\mathbb{E}_{1}^{3}$ are a part of the ruled surfaces of finite type in $\mathbb{E}_{1}^{3}$. We will look for the non-minimal ruled surfaces of finite type in Minkowski 3-space.

We repeat the definition of submanifolds of finite type. A submanifold $M^{n}$ of a Minkowski space $\mathbb{E}_{1}^{n+p}$ is said to be of finite type if each component of its position vector field $X$ can be written as a finite sum of eigenfunctions of the Laplacian $\Delta$ of $M$, that is

$$
X=X_{0}+\sum_{i=1}^{k} X_{i}
$$

where $X_{0}$ is a constant vector and $\Delta X_{i}=\lambda_{i} X_{i} \quad$, for $i=1, \ldots, k$. If in particular all eigenvalues $\left\{\lambda_{1}, \lambda_{2}, \ldots, \lambda_{k}\right\}$ are mutually different, then $M^{n}$ is said to be of $k$-type. Note that every minimal submanifold is of 1-type, since $\Delta X=0$.

A submanifold is of null $k$-type if and only if it is of $k$-type and one of the eigenvalues is equal to zero. 


\subsection{Classification.}

We give a classification of all ruled surfaces of finite type in $\mathbb{E}_{1}^{3}$.

Theorem 3.4. A ruled surface $M$ in $\mathbb{E}_{1}^{3}$ is of finite type if and only if

(1) $M$ is minimal, or

(2) $M$ is a part of a circular cylinder (Figure 14, p. 105), or

(3) $M$ is a part of an hyperbolic cylinder (Figure 15, p. 106), or

(4) $M$ is an isoparametric surface with null rules. (Figures 16-18, p. 107-109)

The examples (2) and (3) are of null 2-type; the example (4) is also of null 2-type, unless it is a one-sheeted hyperboloid, which is of 1-type. Before proving this theorem, we first study non cylindrical ruled surfaces with null rules more in detail.

Theorem 3.5. Let $M$ be a non cylindrical ruled surface in $\mathbb{E}_{1}^{3}$ with null rules, then the following conditions are equivalent

(1) $\frac{g(\dot{\beta}, \dot{\beta})}{g(\dot{\alpha}, \beta)^{2}}=$ nonzero constant ,

(2) the Gaussian curvature $K$ of $M$ is constant,

(3) the mean curvature $H$ of $M$ is constant,

(4) $M$ is isoparametric (in the sense of $[\mathrm{M}]$ ).

Proof. Let $\alpha$ be any regular curve on the surface which intersects the rulings transversally. Let $\beta$ be a differentiable vector field along $\alpha$ such that $\beta(s)$ is pointing in the direction of the ruling through $\alpha(s)$. So we have the parameterization

$$
X(s, t)=\alpha(s)+t \beta(s) .
$$

We assume that $\dot{\beta}$ is never zero. Then $g(\dot{\beta}, \dot{\beta})>0$ for all $s$, and $g(\dot{\alpha}, \beta) \neq 0$, since the surface is non-degenerate. It is easy to check that the function

$$
\rho=\frac{g(\dot{\beta}, \dot{\beta})}{g(\dot{\alpha}, \beta)^{2}}
$$

is independent of the choice of $\alpha$, the parameterization of $\alpha$ or the scaling of $\beta$. A tangent basis is given by

$$
\begin{aligned}
& \frac{\partial X}{\partial s}=\dot{\alpha}+t \dot{\beta}, \\
& \frac{\partial X}{\partial t}=\beta .
\end{aligned}
$$

Then $\xi$ is a unit normal vector, given by

$$
\xi=\frac{-1}{\sqrt{g(\dot{\beta}, \dot{\beta})}}\left(\left(\frac{g(\dot{\alpha}, \dot{\beta})+g(\dot{\beta}, \dot{\beta}) t}{g(\dot{\alpha}, \beta)}\right) \beta-\dot{\beta}\right) .
$$


By straightforward computations we get the shape operator

$$
A_{\xi}=\left(\begin{array}{cc}
\sqrt{\rho} & 0 \\
* & \sqrt{\rho}
\end{array}\right) \text {, }
$$

such that the Gaussian curvature $K$ and the mean curvature $H$ are equal to

$$
\begin{aligned}
& K=\rho \\
& H=\sqrt{\rho} .
\end{aligned}
$$

If $\rho=$ constant, then $M$ is an isoparametric surface [M].

We'll give some nices examples of such surfaces, but first we'll proof theorem 3.4.

Proof of Theorem 3.4 If $M$ is a ruled surface in $\mathbb{E}_{1}^{3}$, i.e. $\mathbb{R}^{3}$ with metric $g=d x_{1}^{2}+d x_{2}^{2}-d x_{3}^{2}$, it can be parameterized as

$$
X(s, t)=\alpha(s)+t \beta(s) .
$$

We consider two cases seperately.

Case 1. $g(\dot{\beta}, \dot{\beta})=0$.

1.1. $\dot{\beta}=0$.

1.1.1. $\beta(s)$ is a constant, spacelike vector. $M$ is a cylinder over a curve $\alpha(s)$ in a timelike plane, which we can choose to have the equation $x_{1}=0$. We can assume that $\alpha$ is parameterised by its arc length, i.e. $g(\dot{\alpha}, \dot{\alpha})=\varepsilon, \quad \varepsilon= \pm 1$. Then a parameterisation $X$ of $M$ is given by

$$
X(s, t)=\alpha(s)+t e_{1} .
$$

The Laplacian $\Delta$ of $M$ is given in terms of $s$ and $t$ by

$$
\Delta=\varepsilon \frac{\partial^{2}}{\partial s^{2}}+\frac{\partial^{2}}{\partial t^{2}}
$$

and the Laplacian $\Delta^{\prime}$ of $\alpha$ is given by

$$
\Delta^{\prime}=\frac{\partial^{2}}{\partial s^{2}} .
$$

Certainly we have that $\Delta t e_{1}=0$. Thus $M$ is of finite type if and only if each component of $\alpha(s)$ can be written as a finite sum of eigenfunctions of $\Delta$, that is,

$$
\alpha(s)=\alpha_{0}+\sum_{i=1}^{k} \alpha_{i}(s, t)
$$

where $\Delta \alpha_{i}=\lambda_{i} \alpha_{i}$. Assume that all the $\lambda_{i}$ are mutually different. If we apply $\prod_{i=2}^{k}(\Delta-$ $\lambda_{i}$ ) to (1), we obtain that $\alpha_{1}$ does not depend on $t$. Similarly we find that none of the $\alpha_{i}$ depend on $t$. Moreover

$$
\varepsilon \Delta^{\prime} \alpha_{i}(s)=\varepsilon \frac{\partial^{2}}{\partial s^{2}} \alpha_{i}(s)=\varepsilon \frac{\partial^{2}}{\partial s^{2}} \alpha_{i}(s)+\frac{\partial^{2}}{\partial t^{2}} \alpha_{i}(s)=\Delta \alpha_{i}(s)=\lambda_{i} \alpha_{i}(s)
$$


for all $i$. Hence every component of $\alpha$ can be written as a finite sum of eigenfunctions of $\Delta^{\prime}$. This means that $\alpha$ is of finite type. Thus $M$ is of finite type if and only if $\alpha$ is of finite type. Moreover, if $\alpha$ is of $k$-type, then $M$ is of $(k+1)$-type, unless one of the eigenfunctions which appear in the decomposition of $\alpha$ has eigenvalue 0 , in which case $M$ is of $k$-type too. The only finite type curves in $\mathbb{E}_{1}^{2}$ are a part of a straight line or a part of an orthogonal hyperbola. So there are only two cases for $M$ in $\mathbb{E}_{1}^{3}$ :

$M$ is a plane (type 1 ), or

$M$ is a cylinder on an orthogonal hyperbola (type 2).

1.1.2. $\beta(s)$ is a constant, timelike vector. Then $M$ is a cylinder over a curve $\alpha(s)$ in a spacelike plane. Similarly as in case 1.1.1. we find that $M$ is of finite type if and only if $\alpha$ is of finite type. Moreover, if $\alpha$ is of $k$-type, then $M$ is of $(k+1)$-type, unless one of the eigenfunctions which appear in the decomposition of $\alpha$ has eigenvalue 0 , in which case $M$ is of $k$-type too. The only finite type curves in $\mathbb{E}^{2}$ [CDVV] are a part of a straight line or a part of a circle. So there are only two cases for $M$ :

$M$ is a plane (type 1 ), or

$M$ is a circular cylinder (type 2).

1.1.3. $\beta(s)$ is a constant nullvector. The Laplacian $\Delta$ of $M$ is given by

$$
\Delta=2 \frac{\partial^{2}}{\partial s \partial t}-\frac{\partial^{2}}{\partial t^{2}}
$$

We see that $\Delta X$ is equal to 0 , so $M$ is a minimal surface.

\section{2. $\dot{\beta} \neq 0$.}

We can decompose $M$ into open pieces such that on each piece we can find a parameterization $X$ of the form

$$
X(s, t)=\alpha(s)+t \beta(s),
$$

where $\alpha$ and $\beta$ are curves in $\mathbb{E}_{1}^{3}$ such that $g(\dot{\alpha}, \beta)=0, g(\beta, \beta)=1$. If we define a function $q$ by

$$
q=g(\dot{\alpha}, \dot{\alpha})+2 \operatorname{tg}(\dot{\alpha}, \dot{\beta})
$$

then it is easy to show that the Laplacian $\Delta$ of $M$ can be expressed as follows

$$
\Delta=\frac{\partial^{2}}{\partial t^{2}}+\frac{1}{q} \frac{\partial^{2}}{\partial s^{2}}-\frac{1}{2} \frac{\partial q}{\partial s} \frac{1}{q^{2}} \frac{\partial}{\partial s}+\frac{1}{2} \frac{\partial q}{\partial t} \frac{1}{q} \frac{\partial}{\partial t} .
$$

The following lemma can be proved by induction.

Lemma 3.6. If $P$ is a polynomial in $t$ with functions in $s$ as coefficients and $\operatorname{deg} P=$ $d$, then

$$
\Delta\left(\frac{P(t)}{q^{m}}\right)=\frac{\tilde{P}(t)}{q^{m+3}}
$$

where $\tilde{P}$ is a polynomial in $t$ with functions in $s$ as coefficients and $\operatorname{deg} \tilde{P} \leq d+2$.

From now on we suppose that $M$ is of $k$-type. Hence there exist numbers $c_{1}, \ldots, c_{k}$; $c_{k} \neq 0$ such that

$$
\Delta^{k+1} X+c_{1} \Delta^{k} X+\ldots+c_{k} \Delta X=0
$$


We know that every component of $X$ is a linear function in $t$ with functions in $s$ as coefficients. By applying the Lemma 3.6, we easily obtain that

$$
\Delta^{r} X=\frac{P_{r}(t)}{q^{3 r}}
$$

where $P_{r}$ is a vector whose components are polynomials in $t$ with functions in $s$ as coefficients and the degree of the components of $P_{r}$ is smaller than or equal to $1+2 r$. Hence if $r$ goes up by one, the degree of the numinator of any component of $\Delta^{r} X$ goes up by at most 2, while the degree of the denominator goes up by 3 . Hence the sum (2) becomes

$$
P_{k+1}(t)+c_{1} P_{k}(t) q^{3}+\ldots+c_{k-1} P_{2}(t) q^{3(k-1)}+c_{k} P_{1}(t) q^{3 k}
$$

this can never be zero because the degree of $P_{1}(t) q^{3 k}$ is always greater then the degree of the other components, unless of course $P_{1}(t)=0$, this means that $\Delta X=0$. But then $M$ is minimal.

Case 2. $g(\dot{\beta}, \dot{\beta}) \neq 0$.

2.1. $g(\beta, \beta) \neq 0$.

We can decompose $M$ into open pieces such that on each piece we can find a parameterization $X$ of the form

$$
X(s, t)=\alpha(s)+t \beta(s),
$$

where $\alpha$ and $\beta$ are curves in $\mathbb{E}_{1}^{3}$ such that $g(\dot{\alpha}, \beta)=0, g(\beta, \beta)=\varepsilon, \quad \varepsilon= \pm 1$. If we define a function $q$ by

$$
q=g(\dot{\alpha}, \dot{\alpha})+2 g(\dot{\alpha}, \dot{\beta}) t+g(\dot{\beta}, \dot{\beta}) t^{2},
$$

then it is easy to show that the Laplacian $\Delta$ of $M$ can be expressed as follows

$$
\Delta=\varepsilon \frac{\partial^{2}}{\partial t^{2}}+\frac{1}{q} \frac{\partial^{2}}{\partial s^{2}}-\frac{1}{2} \frac{\partial q}{\partial s} \frac{1}{q^{2}} \frac{\partial}{\partial s}+\frac{\varepsilon}{2} \frac{\partial q}{\partial t} \frac{1}{q} \frac{\partial}{\partial t} .
$$

The following lemma can also be proved by induction.

Lemma 3.7. If $P$ is a polynomial in $t$ with functions in $s$ as coefficients and $\operatorname{deg} P=$ $d$, then

$$
\Delta\left(\frac{P(t)}{q^{m}}\right)=\frac{\tilde{P}(t)}{q^{m+3}}
$$

where $\tilde{P}$ is a polynomial in $t$ with functions in $s$ as coefficients and $\operatorname{deg} \tilde{P} \leq d+4$.

From now on we suppose that $M$ is of $k$-type. Hence there exist numbers $c_{1}, \ldots, c_{k}$, $c_{k} \neq 0$ such that

$$
\Delta^{k+1} X+c_{1} \Delta^{k} X+\ldots+c_{k} \Delta X=0 .
$$

We know that every component of $X$ is a linear function in $t$ with functions in $s$ as coefficients. By applying the Lemma 3.7, we easily obtain that

$$
\Delta^{r} X=\frac{P_{r}(t)}{q^{3 r}},
$$


where $P_{r}$ is a vector whose components are polynomials in $t$ with functions in $s$ as coefficients and the degree of the components of $P_{r}$ is smaller or equal to $1+4 r$. Hence if $r$ goes up by one, the degree of the numinator of any component of $\Delta^{r} X$ goes up by at most 4 , while the degree of the denominator goes up by 6 . Hence the sum (2) can never be zero, unless of course $\Delta X=0$. But then $M$ is minimal.

\section{2. $g(\beta, \beta)=0$.}

We can decompose $M$ into open pieces such that on each piece we can find a parameterization $X$ of the form

$$
X(s, t)=\alpha(s)+t \beta(s),
$$

where $\alpha$ and $\beta$ are curves in $\mathbb{E}_{1}^{3}$ such that $g(\dot{\beta}, \dot{\beta})>0, g(\beta, \beta)=0$. Because we suppose that $M$ is non-degenerate, we may assume that $g(\dot{\alpha}, \beta) \neq 0$. If we define a function $q$ by

$$
q=g(\dot{\alpha}, \dot{\alpha})+2 g(\dot{\alpha}, \dot{\beta}) t+g(\dot{\beta}, \dot{\beta}) t^{2},
$$

then it is easy to show that the Laplacian $\Delta$ of $M$ can be expressed as follows

$$
\Delta=\frac{1}{g^{2}(\dot{\alpha}, \beta)}\left(-q \frac{\partial^{2}}{\partial t^{2}}+2 g(\dot{\alpha}, \beta) \frac{\partial^{2}}{\partial s \partial t}-\frac{\partial q}{\partial t} \frac{\partial}{\partial t}\right) .
$$

By straightforward computations, we obtain that

$$
\Delta^{r} X=\frac{2^{r-1}}{g^{2}(\dot{\alpha}, \beta)}\left(2(-1)^{r-1} g(\dot{\alpha}, \beta) \frac{\partial}{\partial s}\left(\rho^{r-1} \beta\right)+(-1)^{r} \frac{\partial q}{\partial t}\left(\rho^{r-1} \beta\right)\right),
$$

with

$$
\rho=\frac{g(\dot{\beta}, \dot{\beta})}{g^{2}(\dot{\alpha}, \beta)} .
$$

From now on we suppose that $M$ is of $k$-type. Hence there exist numbers $c_{1}, \ldots, c_{k}$ such that

$$
\Delta^{k+1} X+c_{1} \Delta^{k} X+\ldots+c_{k} \Delta X=0
$$

Expressing that the coefficient by $t$ is equal to null, gives

$$
(-1)^{k+1} 2^{k} \rho^{k}+c_{1}(-1)^{k} 2^{k-1} \rho^{k-1}+\ldots+2 c_{k-1} \rho-c_{k}=0 .
$$

We can conclude that $M$ is of finite type if and only if $\rho$ is constant. Using Theorem 3.5 we know that $M$ is isoparametric.

Conversely, suppose $\rho$ is constant. If we assume that $g(\dot{\alpha}, \beta)=1$, then putting $g(\dot{\beta}, \dot{\beta})=c$, and

$$
\begin{aligned}
& X_{1}(s, t)=\frac{1}{c}(c \alpha-g(\dot{\alpha}, \dot{\beta}) \beta+\dot{\beta}) \\
& X_{2}(s, t)=\frac{1}{c}(c t \beta+g(\dot{\alpha}, \dot{\beta}) \beta-\dot{\beta}) .
\end{aligned}
$$

We obtain that

$$
X(s, t)=X_{1}(s, t)+X_{2}(s, t),
$$


with $\Delta X_{1}=0$ and $\Delta X_{2}=-2 c X_{2}$. Hence $M$ is of null 2-type, unless $X_{1}$ is constant, in which case $M$ is of 1-type. In particular $M$ is then a one-sheeted hyperboloid.So $M$ is of finite type.

Like we mentioned already in the proof, a special case of ruled surfaces of finite type with null rules is the one-sheeted hyperboloid. Indeed, suppose that $g(\alpha, \beta)=0$ ( note that this property does not depend on the choice of $\alpha$ or $\beta)$. Then it is easy to see that

$$
\alpha=b \beta+\frac{g(\alpha, \dot{\beta})}{g(\dot{\beta}, \dot{\beta})} \dot{\beta},
$$

with $b$ an arbitrary function of $s$. Then

$$
X(s, t)=\frac{g(\alpha, \dot{\beta})}{g(\dot{\beta}, \dot{\beta})} \dot{\beta}+(b+t) \beta,
$$

and

$$
g(X, X)=\frac{1}{\rho} .
$$

Using the fact that $M$ is of finite type, gives us a one-sheeted hyperboloid.

On the other hand, we can give a procedure of obtaining at least locally all ruled surfaces of finite type with null rules. We first remark that $\beta$ can always be rescaled and reparameterized (locally) to

$$
\beta(s)=(1, \cos (s), \sin (s)) .
$$

Then $g(\dot{\beta}(s), \dot{\beta}(s))=1$ and $\alpha$ has to satisfy only

$$
g(\dot{\alpha}(s), \beta(s))=c,
$$

for some nonzero constant $c$.

If $\alpha(s)=\left(\alpha_{1}(s), \alpha_{2}(s), \alpha_{3}(s)\right)$, this condition reduces to

$$
\dot{\alpha}_{1}=-c+\dot{\alpha}_{2}(s) \cos (s)+\dot{\alpha}_{3}(s) \sin (s) .
$$

Without loosing generality, we may assume that $\alpha$ is contained in the plane $x_{3}=0$. Putting $\alpha_{2}(s)=f(s)$, we obtain the following formula for the surface :

$$
X(s, t)=\left(-c s+\int \dot{f}(s) \cos (s) d s+t, f(s)+t \cos (s), t \sin (s)\right),
$$

which is, locally, the most general form of a ruled surface of finite type with non parallel null rules.

Using the above formula, we calculate the Laplacian $\Delta$,

$$
\Delta=\frac{1}{c^{2}}\left(2 c \frac{\partial^{2}}{\partial s \partial t}-\frac{\partial q}{\partial t} \frac{\partial}{\partial t}-q \frac{\partial^{2}}{\partial t^{2}}\right),
$$


with

$$
q=\left(-c^{2}+\dot{f}^{2} \sin ^{2}(s)+2 c \dot{f} \cos (s)\right)-2 \dot{f} \sin (s) t+t^{2} .
$$

We'll look for the type of such surfaces, therefore we first calculate

$$
\begin{gathered}
\Delta X(s, t)=\lambda(-\dot{f} \sin (s)+t, c \sin (s)-\dot{f} \sin (s) \cos (s)+\cos (s) t, \\
\left.-c \cos (s)-\dot{f} \sin ^{2}(s)+\sin (s) t\right),
\end{gathered}
$$

with $\lambda=\frac{-2}{c^{2}} \in \mathbb{R}_{0}$.

There are two cases: The first case occurs when

$$
\Delta X(s, t)=\lambda X(s, t)
$$

The surface $M$ is then of type 1 . One of the conditions that must be satisfied is

$$
-c \cos (s)-\dot{f} \sin ^{2}(s)=0 .
$$

After integration and using the other conditions we get that

$$
f(s)=\frac{c}{\sin (s)} .
$$

Then we obtain the parametrization

$$
X(s, t)=\left(c \cot (s)+t, \frac{c}{\sin (s)}+t \cos (s), t \sin (s)\right)
$$

which is a weird parametrization of the hyperboloid.(Figure 2, p. 92)

The second case occurs when

$$
\begin{gathered}
\Delta X(s, t)=\lambda X_{2}(s, t), \\
X_{2}(s, t) \neq X(s, t) .
\end{gathered}
$$

It is then easy to see that

$$
X(s, t)=X_{1}(s, t)+X_{2}(s, t)
$$

with

$$
\begin{aligned}
X_{1}(s, t)=(-c s+\dot{f} \sin (s)+ & \int \dot{f} \cos (s) d s,-c \sin (s)+\dot{f} \sin (s) \cos (s)+f(s), \\
& \left.c \cos (s)+\dot{f} \sin ^{2}(s)\right) .
\end{aligned}
$$

Because $X_{1}$ is only a function of $s$ and using the definition of $\Delta$, we have

$$
\Delta X_{1}=0
$$


$X_{1}$ is a non-constant function, so we have proved that in this case $M$ is of null 2-type.

Some nices examples can be found using the description above, by taking i) $f(s)=0$, we get that

$$
X(s, t)=(t-c s, t \cos (s), t \sin (s)) .
$$

This surface is drawn in Figure 16, p. 107.

ii) $f(s)=s$, we get that

$$
X(s, t)=(t-c s+\sin (s), s+t \cos (s), t \sin (s)) .
$$

This surface is drawn in Figure 17, p. 108.

iii) $f(s)=\sin (s)$, which gives

$$
X(s, t)=\left(t-c s+\frac{1}{2} \sin ^{2}(s),(t-1) \cos (s), t \sin (s)\right) .
$$

This surface is drawn in Figure 18, p. 109. 


\section{§4. RULED SURFACES OF CONSTANT MEAN CURVATURE}

\subsection{Introduction.}

As we saw in the previous section, minimal submanifolds are contained in the class of finite type submanifolds. But that's not the only class they belong to. Moreover they are also a part of the class of submanifolds with constant mean curvature.

A surface $M$ in $\mathbb{E}^{3}$ is of constant mean curvature if and only if

$$
g(H, H)=c,
$$

with $c \in \mathbb{R}, g$ the standard metric in $\mathbb{E}^{3}$ and $H$ the mean curvature vector field. If $c=0$, then $H=0$, which means that $M$ is a minimal surface. Using the formula of Beltrami for surfaces

$$
\Delta X=2 H
$$

where $\Delta$ is the Laplacian and $X$ is the position vector field of $M$. We can say that $M$ is of constant mean curvature if and only if

$$
g(\Delta X, \Delta X)=4 c
$$

The following theorem was proved in [B]

Theorem 4.1. A ruled surface of constant mean curvature in $\mathbb{E}^{3}$ is a minimal surface or a circular cylinder.

Remark that every ruled surface of constant mean curvature in $\mathbb{E}^{3}$ is of finite type and, vice versa.

We'll study the ruled surfaces of constant mean curvature in Minkowski 3-space. The minimal ruled surfaces in $\mathbb{E}_{1}^{3}$ are again a part of this family. We are interested in the non-minimal ruled surfaces with constant mean curvature. We first give a necessary and sufficient condition for a surface in the 3-dimensional Minkowski space $\mathbb{E}_{1}^{3}$ to be of constant mean curvature.

A surface $M$ in a Minkowski space $\mathbb{E}_{1}^{3}$ is of constant mean curvature if and only if

$$
g(H, H)=c,
$$

with $c \in \mathbb{R}, g=-d x_{1}^{2}+d x_{2}^{2}+d x_{3}^{2}$ a metric in $\mathbb{E}_{1}^{3}$ and $H$ the mean curvature vector field. 


\subsection{Classification.}

We will prove the following theorem. if

Theorem 4.2. A ruled surface $M$ in $\mathbb{E}_{1}^{3}$ is of constant mean curvature if and only

(1) $M$ is minimal, or

(2) $M$ is a part of a circular cylinder, (Figure 14, p. 105) or

(3) $M$ is a part of an hyperbolic cylinder, (Figure 15, p. 106) or

(4) $M$ is an isoparametric surface with null rules (Figures 16-18, p. 107-109).

\section{Proof}

Case 1. Suppose that $M$ is a spacelike ruled surface in $\mathbb{E}_{1}^{3}$.

Every spacelike ruled surface $M$ in $\mathbb{E}_{1}^{3}$ can be written as

$$
X(s, t)=\alpha(s)+t \beta(s),
$$

with

$$
g(\beta, \beta)=g(\dot{\alpha}, \dot{\alpha})=1, \quad g(\dot{\alpha}, \beta)=0 .
$$

$\alpha(s)$ is a spacelike curve in $\mathbb{E}_{1}^{3}$ with arc length parameter $s$ and $\beta$ is a unit normal vector field along $\alpha$. We have that

$$
g_{i j}=\left(\begin{array}{ll}
q & 0 \\
0 & 1
\end{array}\right)
$$

and

$$
g^{i j}=\left(\begin{array}{cc}
\frac{1}{q} & 0 \\
0 & 1
\end{array}\right)
$$

with $q=1+2 g(\dot{\alpha}, \dot{\beta}) t+g(\dot{\beta}, \dot{\beta}) t^{2}$. The Laplacian $\Delta$ of $M$ is given by

$$
\Delta=\frac{-1}{2 q^{2}} \frac{\partial q}{\partial s} \frac{\partial}{\partial s}+\frac{1}{q} \frac{\partial^{2}}{\partial s^{2}}+\frac{1}{2 q} \frac{\partial q}{\partial t} \frac{\partial}{\partial t}+\frac{\partial^{2}}{\partial t^{2}}
$$

and

$$
\Delta X=\frac{-1}{2 q^{2}} \frac{\partial q}{\partial s}(\dot{\alpha}+t \dot{\beta})+\frac{1}{q}(\ddot{\alpha}+t \ddot{\beta})+\frac{1}{2 q} \frac{\partial q}{\partial t} \beta .
$$

Expressing that $M$ is of constant mean curvature, is equivalent with

$$
g(\Delta X, \Delta X)=c
$$

with $c \in \mathbb{R}$. $M$ is spacelike, so we know that $c \leq 0$ and moreover that $c=0$ only when $H=0$, thus when $M$ is minimal. Computing $g(\Delta X, \Delta X)=c$ gives us the following polynomial in $t$ that must be equal to 0 ,

$$
\left(\frac{\partial q}{\partial s}\right)^{2}+4 q g(\ddot{\alpha}+t \ddot{\beta}, \ddot{\alpha}+t \ddot{\beta})+q\left(\frac{\partial q}{\partial t}\right)^{2}+4 q \frac{\partial q}{\partial t} g(\ddot{\alpha}+t \ddot{\beta}, \beta)-4 \frac{\partial q}{\partial s} g(\dot{\alpha}+t \dot{\beta}, \ddot{\alpha}+t \ddot{\beta})-4 c q^{3} .
$$


Putting the coefficient by $t^{6}$ equal to 0 , we get the following condition,

$$
g(\dot{\beta}, \dot{\beta})=0
$$

There are two cases:

i) $\dot{\beta}=0$

In this case,

$$
\Delta=\frac{\partial^{2}}{\partial s^{2}}+\frac{\partial^{2}}{\partial t^{2}}
$$

and

$$
\Delta X=\ddot{\alpha}
$$

so

$$
g(\Delta X, \Delta X)=g(\ddot{\alpha}, \ddot{\alpha})=c<0 .
$$

$\beta$ is a constant, spacelike vector. Without loss of generality, we can take

$$
\beta(s)=(0,0,1) \text {. }
$$

From $g(\dot{\alpha}, \beta)=0$, follows that

$$
\dot{\alpha}(s)=\left(\dot{\alpha}_{1}(s), \dot{\alpha}_{2}(s), 0\right) .
$$

$s$ is the arc length parameter for $\alpha$, so

$$
\dot{\alpha}_{1}(s)=\sinh (f(s)), \quad \dot{\alpha}_{2}(s)=\cosh (f(s)),
$$

with $f(s)$ an arbitrary function. Using the condition mentioned above for $\ddot{\alpha}$, we obtain

$$
f(s)=\sqrt{-c} s+b, \quad b \in \mathbb{R} .
$$

After integration and translation,

$$
\alpha(s)=\left(\frac{1}{\sqrt{-c}} \cosh (\sqrt{-c} s), \frac{1}{\sqrt{-c}} \sinh (\sqrt{-c} s), 0\right),
$$

which means that $M$ is a part of a spacelike hyperbolic cylinder.

ii) $\dot{\beta} \neq 0$

From $g(\beta, \ddot{\beta})=0$ and $g(\beta, \dot{\beta})=0$ follows that $\dot{\beta}$ and $\ddot{\beta}$ lie in the same timelike plane. From $g(\dot{\beta}, \dot{\beta})=0$ and $g(\dot{\beta}, \ddot{\beta})=0$ follows that

$$
\ddot{\beta}(s)=\lambda \dot{\beta}, \quad \lambda \in \mathbb{R}
$$

and

$$
g(\ddot{\beta}, \ddot{\beta})=0 .
$$

If $M$ is of constant mean curvature then

$$
g(\Delta X, \Delta X)=c,
$$


which gives in this case a polynomial of degree 3 . The coefficient by $t^{3}$ must be 0 . This is - after some computations - equivalent with

$$
g(\dot{\alpha}, \dot{\beta})\left[g(\ddot{\beta}, \ddot{\beta})-4 c g^{2}(\dot{\alpha}, \dot{\beta})\right]=0 .
$$

With $g(\ddot{\beta}, \ddot{\beta})=0$ and $c<0$, we obtain that

$$
g(\dot{\alpha}, \dot{\beta})=0
$$

This gives a contradiction, because $\dot{\beta}$ is then perpendicular to the spacelike plane $\{\dot{\alpha}, \beta\}$.

Case 2. $M$ is a Lorentzian ruled surface

2.a. The rules are spacelike.

Every Lorentz ruled surface $M$ with spacelike rules can be written as

$$
X(s, t)=\alpha(s)+t \beta(s),
$$

with

$$
g(\beta, \beta)=1, \quad g(\dot{\alpha}, \dot{\alpha})=-1, \quad g(\dot{\alpha}, \beta)=0 .
$$

$\alpha(s)$ is a timelike curve in $\mathbb{E}_{1}^{3}$ with arc length parameter $s$ and $\beta$ is a unit normal vector field along $\alpha$. We have that

$$
g_{i j}=\left(\begin{array}{ll}
q & 0 \\
0 & 1
\end{array}\right)
$$

and

$$
g^{i j}=\left(\begin{array}{cc}
\frac{1}{q} & 0 \\
0 & 1
\end{array}\right)
$$

with $q=-1+2 g(\dot{\alpha}, \dot{\beta}) t+g(\dot{\beta}, \dot{\beta}) t^{2}$.

The Laplacian $\Delta$ is given by

$$
\Delta=\frac{-1}{2 q^{2}} \frac{\partial q}{\partial s} \frac{\partial}{\partial s}+\frac{1}{q} \frac{\partial^{2}}{\partial s^{2}}+\frac{1}{2 q} \frac{\partial q}{\partial t} \frac{\partial}{\partial t}+\frac{\partial^{2}}{\partial t^{2}}
$$

and

$$
\Delta X=\frac{-1}{2 q^{2}} \frac{\partial q}{\partial s}(\dot{\alpha}+t \dot{\beta})+\frac{1}{q}(\ddot{\alpha}+t \ddot{\beta})+\frac{1}{2 q} \frac{\partial q}{\partial t} \beta .
$$

Expressing that $M$ is of constant mean curvature, is equivalent with

$$
g(\Delta X, \Delta X)=c
$$

with $c \in \mathbb{R} . M$ is timelike, so we know that $c \geq 0$ and moreover that $c=0$ only when $H=0$, thus when $M$ is minimal. Computing $g(\Delta X, \Delta X)=c$ gives us the following polynomial in $t$ that must be equal to 0 ,

$$
\left(\frac{\partial q}{\partial s}\right)^{2}+4 q g(\ddot{\alpha}+t \ddot{\beta}, \ddot{\alpha}+t \ddot{\beta})+q\left(\frac{\partial q}{\partial t}\right)^{2}+4 q \frac{\partial q}{\partial t} g(\ddot{\alpha}+t \ddot{\beta}, \beta)-4 \frac{\partial q}{\partial s} g(\dot{\alpha}+t \dot{\beta}, \ddot{\alpha}+t \ddot{\beta})-4 c q^{3} .
$$


Putting the coefficient by $t^{6}$ equal to 0 , we get the following condition,

$$
g(\dot{\beta}, \dot{\beta})=0
$$

There are again two cases :

i) $\dot{\beta}=0$

In this case,

$$
\Delta=\frac{\partial^{2}}{\partial s^{2}}+\frac{\partial^{2}}{\partial t^{2}}
$$

and

$$
\Delta X=\ddot{\alpha}
$$

so

$$
g(\Delta X, \Delta X)=g(\ddot{\alpha}, \ddot{\alpha})=c>0 .
$$

$\beta$ is a constant, spacelike vector. Without loss of generality, we can take

$$
\beta(s)=(0,0,1) \text {. }
$$

From $g(\dot{\alpha}, \beta)=0$ follows that

$$
\dot{\alpha}(s)=\left(\dot{\alpha}_{1}(s), \dot{\alpha}_{2}(s), 0\right),
$$

$s$ is the arc length parameter for $\alpha$ so

$$
\dot{\alpha}_{1}(s)=\cosh (f(s)), \quad \dot{\alpha}_{2}(s)=\sinh (f(s)),
$$

with $f(s)$ an arbitrary function. Using the condition mentioned above for $\ddot{\alpha}$, we obtain

$$
f(s)=\sqrt{c} s+b, \quad b \in \mathbb{R} .
$$

After integration and translation,

$$
\alpha(s)=\left(\frac{1}{\sqrt{c}} \sinh (\sqrt{c} s), \frac{1}{\sqrt{c}} \cosh (\sqrt{c} s), 0\right),
$$

which means that $M$ is a part of a timelike hyperbolic cylinder.

ii) $\dot{\beta} \neq 0$

From $g(\beta, \ddot{\beta})=0$ and $g(\beta, \dot{\beta})=0$ follows that $\dot{\beta}$ and $\ddot{\beta}$ lie in the same timelike plane. From $g(\dot{\beta}, \dot{\beta})=0$ and $g(\dot{\beta}, \ddot{\beta})=0$ follows that

$$
\ddot{\beta}(s)=\lambda \dot{\beta}, \quad \lambda \in \mathbb{R}
$$

and

$$
g(\ddot{\beta}, \ddot{\beta})=0 .
$$

If $M$ is of constant mean curvature then

$$
g(\Delta X, \Delta X)=c,
$$


which gives in this case a polynomial of degree 3 . The coefficient by $t^{3}$ must be 0 . This is - after some computations - equivalent with

$$
g(\dot{\alpha}, \dot{\beta})\left[g(\ddot{\beta}, \ddot{\beta})-4 c g^{2}(\dot{\alpha}, \dot{\beta})\right]=0 .
$$

With $g(\ddot{\beta}, \ddot{\beta})=0$ and $c>0$, we obtain that

$$
g(\dot{\alpha}, \dot{\beta})=0
$$

This gives a contradiction, because $\dot{\beta}$ is then perpendicular to the timelike plane $\{\dot{\alpha}, \beta\}$.

2.b. The rules are timelike.

Every Lorentz ruled surface $M$ with timelike rules can be written as

$$
X(s, t)=\alpha(s)+t \beta(s),
$$

with

$$
g(\beta, \beta)=-1, \quad g(\dot{\alpha}, \dot{\alpha})=1, \quad g(\dot{\alpha}, \beta)=0 .
$$

$\alpha(s)$ is a spacelike curve in $\mathbb{E}_{1}^{3}$ with arc length parameter $s$ and $\beta$ is a unit normal vector field along $\alpha$. We have that

$$
g_{i j}=\left(\begin{array}{cc}
q & 0 \\
0 & -1
\end{array}\right)
$$

and

$$
g^{i j}=\left(\begin{array}{cc}
\frac{1}{q} & 0 \\
0 & -1
\end{array}\right)
$$

with $q=1+2 g(\dot{\alpha}, \dot{\beta}) t+g(\dot{\beta}, \dot{\beta}) t^{2}$. The only difference with the previous case occurs when $\dot{\beta}=0$.

In this case,

$$
\Delta=\frac{\partial^{2}}{\partial s^{2}}-\frac{\partial^{2}}{\partial t^{2}}
$$

and

$$
\Delta X=\ddot{\alpha}
$$

so

$$
g(\Delta X, \Delta X)=g(\ddot{\alpha}, \ddot{\alpha})=c>0 .
$$

$\beta$ is a constant, timelike vector. Without loss of generality, we can take

$$
\beta(s)=(1,0,0) \text {. }
$$

From $g(\dot{\alpha}, \beta)=0$ follows that

$$
\dot{\alpha}(s)=\left(0, \dot{\alpha}_{2}(s), \dot{\alpha}_{3}(s)\right),
$$

$s$ is the arc length parameter for $\alpha$ so

$$
\dot{\alpha}_{2}(s)=\cos (f(s)), \quad \dot{\alpha}_{3}(s)=-\sin (f(s)),
$$


with $f(s)$ an arbitrary function. Using the condition mentioned above for $\ddot{\alpha}$, we obtain

$$
f(s)=\sqrt{c} s+b, \quad b \in \mathbb{R} .
$$

After integration and translation,

$$
\alpha(s)=\left(0, \frac{1}{\sqrt{c}} \sin (\sqrt{c} s), \frac{1}{\sqrt{c}} \cos (\sqrt{c} s)\right),
$$

which means that $M$ is a part of a timelike circular cylinder.

2.c. The rules are null.

Every Lorentzian ruled surface $M$ with null rules can be written as

$$
X(s, t)=\alpha(s)+t \beta(s),
$$

with

We have that

$$
g(\beta, \beta)=0, \quad g(\dot{\alpha}, \beta) \neq 0
$$

and

$$
g_{i j}=\left(\begin{array}{cc}
q & g(\dot{\alpha}, \beta) \\
g(\dot{\alpha}, \beta) & 0
\end{array}\right)
$$

$$
g^{i j}=\left(\begin{array}{cc}
0 & \frac{1}{g(\dot{\alpha}, \beta)} \\
\frac{1}{g(\dot{\alpha}, \beta)} & \frac{-q}{g^{2}(\dot{\alpha}, \beta)}
\end{array}\right) .
$$

with $q=g(\dot{\alpha}, \dot{\alpha})+2 g(\dot{\alpha}, \dot{\beta}) t+g(\dot{\beta}, \dot{\beta}) t^{2}$. Using the definition of $\Delta$, we get

$$
\Delta X=\frac{1}{g^{2}(\dot{\alpha}, \beta)}\left[2 g(\dot{\alpha}, \beta) \frac{\partial^{2} X}{\partial t \partial s}-\frac{\partial q}{\partial t} \frac{\partial X}{\partial t}-q \frac{\partial^{2} X}{\partial t^{2}}\right],
$$

in casu

$$
\Delta X=\frac{1}{g^{2}(\dot{\alpha}, \beta)}\left[2 g(\dot{\alpha}, \beta) \dot{\beta}-\frac{\partial q}{\partial t} \beta\right] .
$$

So,

$$
g(\Delta X, \Delta X)=c>0
$$

corresponds with

$$
\frac{g(\dot{\beta}, \dot{\beta})}{g^{2}(\dot{\alpha}, \beta)}=\rho=\frac{c}{4} \in \mathbb{R}_{0}^{+} .
$$

Using Theorem 3.5. we obtain that the Gaussian curvature

$$
K=\rho
$$

and the mean curvature

$$
H=\sqrt{\rho}
$$

are constant. This means that $M$ is an isoparametric surface [M].

Remark that also in the 3 -dimensional Minkowski space every ruled surface with constant mean curvature of finite type is and vice versa.

For a classification and examples of isoparametric surfaces with null rules, we refer to the end of the previous paragraph. 
CHAPTER III

\section{TENSOR PRODUCT SURFACES OF A LORENTZIAN AND A EUCLIDEAN CURVE}

\section{§1. INTRODUCTION}

The origin of the study of tensor product submanifolds is related with the study of minimal submanifolds in spheres. It was the well-known result of $\mathrm{T}$. Takahashi [Ta] about 1 type submanifolds in $\mathbb{E}^{m}$, that urged several mathematicians to look for all minimal submanifolds in spheres.

Theorem 1.1. A submanifold $M^{n}$ of $\mathbb{E}^{m}$ is of 1 type if and only if either $M$ is a minimal submanifold of the ambient Euclidean space $\mathbb{E}^{m}$ or $M$ is a minimal submanifold of some hypersphere $S^{m-1}$ in $\mathbb{E}^{m}$.

The class of minimal submanifolds in spheres is very large. Hence, if one aims for classification results, one needs to impose additional conditions on such submanifolds. One of this conditions can be stated in terms of the Chen type of their quadratic representation. According to S.S. Tai [Tai], the second standard minimal immersion of a sphere

$$
S^{m}(r)=\left\{x=\left(x_{1}, x_{2}, \ldots, x_{m+1}\right) \in \mathbb{E}^{m+1} \mid x_{1}^{2}+x_{2}^{2}+\ldots+x_{m+1}^{2}=r^{2}\right\}
$$

can essentially be described by

$$
\begin{gathered}
f: S^{m}(r) \rightarrow S M(m+1) \\
x \rightarrow x^{t} \cdot x=\left(\begin{array}{c}
x_{1} \\
x_{2} \\
\cdot \\
\cdot \\
x_{m+1}
\end{array}\right) \cdot\left(x_{1}, x_{2}, \ldots, x_{m+1}\right),
\end{gathered}
$$

whereby $S M(m+1)$ denotes the set of all real symmetric matrices of order $m+1$, which can be identified naturally with $\mathbb{R}^{\frac{1}{2}(m+1)(m+2)}$, and becomes a Euclidean space with the metric

$$
<A, B>:=\frac{1}{r^{2}} \cdot \operatorname{tr}(A B),
$$

with $A, B \in S M(m+1)$.

Then, given a spherical submanifold $x: M^{n} \rightarrow S^{m}(r) \subset \mathbb{E}^{m+1}$, the map

$$
\phi: M^{n} \stackrel{x}{\longrightarrow} S^{m}(r) \stackrel{f}{\longrightarrow} S M(m+1)
$$


is called the quadratic representation of the submanifold $M^{n}$. Various authors, beginning with A. Ros [Ros], studied minimal submanifolds in spheres under an additional condition on the finite Chen type of their quadratic representation. In particular, in this respect, we mention here the following results of $\mathrm{M}$. Barros and B.Y. Chen $[\mathrm{BC}]$ and of $\mathrm{M}$. Barros and F. Urbano [BU], which characterize some of the nicest submanifolds among the minimal surfaces in spheres.

Theorem 1.2. Let $M$ be a minimal surface of a hypersphere $S^{m}(1)$. Then, the quadratic representation $\phi$ of $M$ is :

(1) of 1 type if and only if $M$ is a totally geodesic $S^{2}$ in $S^{3}(1)$;

(2) of 2 type if and only if $M$ is a Veronese surface in $S^{4}(1)$ or a Clifford torus in $S^{3}(1)$

(3) of 3 type if and only if $M$ is a Veronese surface in $S^{6}(1)$ or an equilateral torus in $S^{5}(1)$.

More generally, B.Y. Chen considered the quadratic representation $\tilde{x}$ of Euclidean submanifolds $x: M \rightarrow \mathbb{E}^{m}$ ( thus extending $\phi$ from spherical submanifolds $x: M \rightarrow$ $S^{m} \subset \mathbb{E}^{m+1}$ to arbitrary submanifolds in Euclidian spaces). For a given submanifold

$$
x: M \rightarrow \mathbb{E}^{m}: p \rightarrow x(p)=\left(x_{1}(p), x_{2}(p), \ldots, x_{m}(p)\right),
$$

its quadratic representation is defined by

$$
\tilde{x}: M \rightarrow \mathbb{E}^{\frac{1}{2} m(m+1)}: p \rightarrow \tilde{x}(p)=\left(x_{1}^{2}, \ldots, x_{m}^{2}, x_{1} x_{2}, \ldots, x_{m-1} x_{m}\right)(p)
$$

whereby the matrix product

$$
\left(x^{t} \cdot x\right)(p)=\left(\begin{array}{c}
x_{1}(p) \\
\cdot \\
\ddots \\
x_{m+1}(p)
\end{array}\right) \cdot\left(x_{1}(p), \ldots, x_{m+1}(p)\right)
$$

in $S M(m)$ is naturally identified with $\tilde{x}(p)$ in $\mathbb{E}^{\frac{1}{2} m(m+1)}$.

B.Y. Chen initiated the study of the tensor product immersion of two immersions of a given Riemannian manifold; this concept orginated from the investigation of the quadratic representation of submanifolds.

Let $V$ and $W$ be two vector spaces over the field of the real numbers $\mathbb{R}$, endowed with inner products $g_{V}($,$) and g_{W}($,$) respectively. Then both the direct sum V \oplus W$ and the tensor product $V \otimes W$ are inner product spaces with inner products defined by

$$
\begin{gathered}
g_{V \oplus W}(v \oplus w, \dot{v} \oplus \dot{w})=g_{V}(v, \dot{v})+g_{W}(w, \dot{w}), \\
g_{V \otimes W}(v \otimes w, \dot{v} \otimes \dot{w})=g_{V}(v, \dot{v}) . g_{W}(w, \dot{w}),
\end{gathered}
$$

for any $v, \dot{v} \in V$ and $w, \dot{w} \in W$.

In particular, for Euclidean spaces, one has the natural isomorphisms

$$
\mathbb{E}^{p} \oplus \mathbb{E}^{q} \cong \mathbb{E}^{p+q}
$$


and

$$
\mathbb{E}^{p} \otimes \mathbb{E}^{q} \cong \mathbb{E}^{p q} \text {. }
$$

By applying these algebraic notions, we can define the direct sum and tensor product maps of two immersions of a given Riemannian manifold.

Let $(M, g)$ be a Riemannian manifold and

$$
\begin{aligned}
& f: M \rightarrow \mathbb{E}^{m}, \\
& h: M \rightarrow \mathbb{E}^{n},
\end{aligned}
$$

two immersions of $M$ into Euclidean spaces. Then

$$
\begin{gathered}
f \oplus h: M \rightarrow \mathbb{E}^{m+n}: x \rightarrow(f(x), h(x)), \\
f \otimes h: M \rightarrow \mathbb{E}^{m n}: x \rightarrow f(x) \otimes h(x),
\end{gathered}
$$

for any $x \in M$.

Remark. For a Euclidean immersion $i: M \rightarrow \mathbb{E}^{m}$, the tensor product $i \otimes i$ is essentially the quadratic representation of $M$, as studied by A. Ros, B.Y. Chen, M. Barros, O. Garay, F. Urbano and I. Dimitric.

In [Ch], B.Y. Chen defines the tensor product field of $f_{1} \otimes \ldots \otimes f_{k}$. Let $f_{i}: M \rightarrow$ $\mathbb{E}^{m_{i}}(i=1, \ldots, k)$ be $k$ immersions from a Riemannian manifold $(M, g)$ into Euclidean spaces. The tensor product vector field $X_{f_{1} \otimes \ldots \otimes f_{k}}$ on $M$ is defined by

$$
\begin{gathered}
X_{f_{1} \otimes \ldots \otimes f_{k}}= \\
\sum_{i<j} \sum_{l=1}^{n} f_{1} \otimes \ldots f_{i-1} \otimes d f_{i}\left(e_{l}\right) \otimes \ldots \otimes f_{j-1} \otimes d f_{j}\left(e_{l}\right) \otimes \ldots \otimes f_{k},
\end{gathered}
$$

where $\left\{e_{1}, \ldots, e_{n}\right\}$ is an orthonormal local frame field on $M$.

Inspired by Chen's definition, F. Decruyenaere, F. Dillen, L. Vrancken and L. Verstraelen studied the tensor product of two immersions of, in general, different manifolds ([DDVV]); under some conditions, this realizes an immersion of the product manifold.

\section{Definition 1.3. [DDVV]} that

Let $M$ and $N$ be two differentiable manifolds of dimension $r$, resp. $s$, and assume

$$
f: M \rightarrow \mathbb{E}^{m}
$$

and

$$
h: N \rightarrow \mathbb{E}^{n}
$$

are two immersions. Then the direct sum and tensor product maps are defined respectively by

$$
f \oplus h: M \times N \rightarrow \mathbb{E}^{m+n}:(p, q) \rightarrow(f(p), h(p))=\left(f^{1}(p), \ldots, f^{m}(p), h^{1}(q), \ldots, h^{n}(q)\right),
$$

and

$f \otimes h: M \times N \rightarrow \mathbb{E}^{m n}:(p, q) \rightarrow f(p) \otimes h(q)=\left(f^{1}(p) h^{1}(q), \ldots, f^{1}(p) h^{n}(q), \ldots, f^{m}(p) h^{n}(q)\right)$.

Necessary and sufficient conditions for $f \otimes h$ to be an immersion were obtained in [DDVV]. 
Proposition 1.4. The tensor product $f \otimes h$ is an immersion if and only if $f$ and $h$ are immersions that satisfy the three following conditions :

(1) either $f$ or $h$ is transversal;

(2) $0 \notin \operatorname{Im}(f)$;

(3) $0 \notin \operatorname{Im}(h)$.

An immersion $f: M \rightarrow \mathbb{E}^{m}$ is said to be transversal in a point $p \in M$ if and only if the position vector $f(p)$ is not tangent to $M$ at $p$. If $f$ is transversal in every point of $M$, then $f$ shortly is called transversal. If $f$ and $h$ are transversal immersions, then their tensor product is a transversal immersion too (see [DDVV]). But, for many immersions which are not transversal, the tensor product $f \otimes h$ is still worthwhile to be investigated and in many cases still produces an immersion.

Interesting examples of tensor product immersions are provided by the rotation surfaces of Vrănceanu. We recall that these surfaces are given by the parameterisations

$$
X(u, v)=(r(v) \cos (u) \cos (v), r(v) \cos (u) \sin (v), r(v) \sin (u) \cos (v), r(v) \sin (u) \sin (v))
$$

or in terms of the tensor product

$$
X(u, v)=(\cos (u), \sin (u)) \otimes(r(v) \cos (v), r(v) \sin (v)) .
$$

C.S. Houh proved that such a surface is of finite type if and only if it is a Clifford torus, i.e. it is the product of two plane circles with the same radius.

The tensor product surfaces of two Euclidean plane curves were studied by I. Mihai, R. Rosca, L. Verstraelen and L. Vrancken [MRVV] and by I. Mihai and B. Rouxel [MR]. We recall the definition and the main results.

Definition 1.5.

Let $\alpha: \mathbb{R} \rightarrow \mathbb{E}^{2}$ and $\beta: \mathbb{R} \rightarrow \mathbb{E}^{2}$ be two Euclidean planar curves. Put $\alpha(t)=$ $\left(\alpha_{1}(t), \alpha_{2}(t)\right)$ and $\beta(s)=\left(\beta_{1}(s), \beta_{2}(s)\right)$. Then their tensor product is given by

$$
\begin{gathered}
f=\alpha \otimes \beta: \mathbb{R}^{2} \rightarrow \mathbb{E}^{4} \\
f(t, s)=\left(\alpha_{1}(t) \beta_{1}(s), \alpha_{1}(t) \beta_{2}(s), \alpha_{2}(t) \beta_{1}(s), \alpha_{2}(t) \beta_{2}(s)\right) .
\end{gathered}
$$

The first theorem from [MRVV] classifies all the minimal tensor product surfaces.

Theorem 1.6. The tensor product immersion $\alpha \otimes \beta$ of two Euclidean planar curves is a minimal surface in $\mathbb{E}^{4}$ if and only if either

(1) $\alpha$ is a portion of a straight line through 0 ;

(2) $\beta$ is a portion of a straight line through 0 ;

(3) $\alpha$ is a circle centered at 0 and $\beta$ is an orthogonal hyperbola centered at 0 , or vice versa.

Before we give some more results, we first repeat some useful definitions.

In terms of the behaviour of the tangent bundle $T M$ of the submanifold $M$, a totally real submanifold $M$ of an almost Hermitian manifold $N$ is a submanifold such that the 
complex structure $J$ of the ambient manifold $N$ carries each tangent vector of $M$ into the corresponding normal space of $M$ in $N$. That means,

$$
J\left(T_{p} M\right) \subseteq T_{p}^{\perp} M
$$

for any point $p \in M$. In other words, $M$ is a totally real submanifold of $(N, g, J)$ if and only if for any nonzero vector $X$ tangent to $M$ at any point $p \in M$, the angle between $J X$ and the tangent space $T_{p} M$ is equal to $\frac{\pi}{2}$, identically. The study of totally real submanifolds from the differential geometric points of view was initiated in the early 1970's. Since then many differential geometers have contributed many interesting results in this subject.

The complex submanifolds $M$ of an almost Hermitian manifold $(N, g, J)$ are characterized by the condition

$$
J\left(T_{p} M\right) \subseteq T_{p} M
$$

for any point $p \in M$. In other words, $M$ is a complex submanifold of $(N, g, J)$ if and only if for any nonzero vector $X$ tangent to $M$ at any point $p \in M$, the angle between $J X$ and the tangent space $T_{p} M$ is equal to zero, identically.

The third important class of submanifolds of an almost Hermitian manifold $(N, g, J)$ are slant submanifolds. A slant submanifold $M$ is defined as a submanifold of $(N, g, J)$ such that, for any nonzero vector $X$ tangent to $M$, the angle $\theta(X)$ between $J X$ and the tangent space $T_{p} M$ is a constant, which is independent of the choice of the point $p \in M$ and the choice of the tangent vector $X$ in the tangent space $T_{p} M$. It is obvious that totally real submanifolds and complex submanifolds are special classes of slant submanifolds. A slant submanifold is called proper slant if it is neither a totally real submanifold nor a complex submanifold. For more information about proper slant submanifolds, we refer to [Ch1].

The following three theorems determine the totally real, complex and proper slant tensor product surfaces in $\mathbb{E}^{4}$.

Theorem 1.7. The tensor product immersion $\alpha \otimes \beta$ of two Euclidean planar curves is totally real in $\left(\mathbb{C}^{2}, J\right)$, with $J$ the standard complex structure, if and only if $\alpha$ is a portion of a straight line which contains 0 or $\beta$ is a portion of a circle centered at 0 .

Theorem 1.8. The tensor product immersion $\alpha \otimes \beta$ of two Euclidean planar curves is complex with respect to at least one complex structure on $\mathbb{C}^{2}$ if and only if either

(1) $\alpha$ is a portion of a straight line through 0 ;

(2) $\beta$ is a portion of a straight line through 0 ;

(3) $\alpha$ is a circle centered at 0 and $\beta$ is an orthogonal hyperbola centered at 0 , or vice versa.

Remark that all complex surfaces are minimal and vice versa. 
Theorem 1.9. The tensor product immersion $\alpha \otimes \beta$ of two Euclidean planar curves is a proper slant surface in $\left(\mathbb{C}^{2}, J\right)$ if and only if $\beta$ is a logarithmic spiral and $\alpha$ is either a circle centered at 0 or a logarithmic spiral too.

Just like a point $p \in M$ is called umbilic if the principal curvatures for $\xi$ are all equal, for every $\xi \in T_{p}^{\perp} M$. In other words, the shape operator with respect to $\xi$ must be some multiple of the identity, so for each $\xi$ there must be a $\lambda$ with

$$
A_{\xi}(X)=\lambda X
$$

or equivalently

$$
<h(X, Y), \xi\rangle=\lambda\langle X, Y\rangle, \quad \text { for all } X, Y \in T_{p} M \text {. }
$$

Just in the same way, an isometric immersion $f$ is said to be pseudo-umbilical if the shape operator with respect to $H$ is proportional to the identity. It is obvious that every minimal submanifold is pseudo-umbilical.

A general classification of all non-minimal pseudo-umbilical tensor product surfaces in $\mathbb{E}^{4}$ is not found, but a special case gives the following result.

Theorem 1.10. The tensor product of a circle centered at 0 and a Euclidean planar curve is a non-minimal pseudo-umbilical surface in $\mathbb{E}^{4}$ if and only if it is a rotation surface of Vrănceanu, with

$$
r(s)=k e^{b s}, \quad k, b \in \mathbb{R}, k \neq 0
$$

i.e. if and only if the second Euclidean plane curve is either a circle centered at the origin or a logarithmic spiral.

In $[M R]$ the Kommerell conic of a surface in $\mathbb{E}^{4}$ was used to study tensor product surfaces of Euclidean plane curves. We give some of the main results. if

Theorem 1.11. The tensor product of two Euclidean plane curves is flat if and only

(1) the curves are two spiral sinusoids

$$
\begin{aligned}
& \alpha(t)=\left((\cos (h t))^{1 / h} \cos (t),(\cos (h t))^{1 / h} \sin (t)\right), \\
& \beta(s)=\left((\cos (k s))^{1 / k} \cos (s),(\cos (k s))^{1 / k} \sin (s)\right),
\end{aligned}
$$

with $\frac{1}{h}+\frac{1}{k}=-1$, or

(2) the curves are two logarithmic spiral curves. 
Theorem 1.12. The tensor product surface $\alpha \otimes \beta$ of two Euclidean plane curves is a ruled surface if and only if $\alpha$ or $\beta$ is a straight line.

Another class of submanifolds are the so called Chen submanifolds (see [C]). A submanifold $M$ of a Riemannian manifold $N$ is called a Chen submanifold of $N$ if the allied mean curvature vector of $M$ in $N$ vanishes identically.

The allied mean curvature vector of an n-dimensional submanifold $M$ of an mdimensional Riemannian manifold $N$ is defined as follows. Let $e_{n+1}, \ldots, e_{m}$ be mutually orthogonal unit normal vector fields of $M$ in $N$ such that $e_{n+1}$ is parallel to the mean curvature vector $H$ of $\operatorname{Min} N$. We define a normal vector field $a(H)$ by

$$
a(H)=\sum_{r=n+2}^{m} \operatorname{tr}\left(A_{H} A_{e_{r}}\right) e_{r}
$$

Then $a(H)$ is a well-defined normal vector field (up to sign) of $M$ in $N$. We call $a(H)$ the allied mean curvature vector of $M$ in $N$. It is clear that $a(H)$ is perpendicular to $H$.

B.Rouxel showed that all rotation surfaces of Vrănceanu are Chen surfaces.

Theorem 1.13. The tensor product surface $\alpha \otimes \beta$ of two Euclidean plane curves is a non-trivial Chen surface

(1) if $\alpha$ or $\beta$ is a circle with center 0 and $\alpha \otimes \beta$ is a rotation surface of Vrănceanu,

(2) $\alpha$ is a straight line and $\beta$ a circle through 0 (or vice versa); $\alpha \otimes \beta$ is then a ruled Chen surface.

For the tensor product surfaces of two Lorentzian curves we refer to [MVVW] and the additional thesis of I.Mihai [M]. 


\section{§2. MINIMAL TENSOR PRODUCT SURFACES}

In this section we classify minimal tensor product surfaces of a Lorentzian planar curve and a Euclidean planar curve.

Let $\alpha: \mathbb{R} \rightarrow \mathbb{E}_{1}^{2}(-+)$ and $\beta: \mathbb{R} \rightarrow \mathbb{E}^{2}$ be respectively a Lorentzian planar curve and a Euclidean planar curve. Put $\alpha(t)=\left(\alpha_{1}(t), \alpha_{2}(t)\right)$ and $\beta(s)=\left(\beta_{1}(s), \beta_{2}(s)\right)$. Then their tensor product is given by

$$
\begin{gathered}
f=\alpha \otimes \beta: \mathbb{R}^{2} \rightarrow \mathbb{E}_{2}^{4}(--++) \\
f(t, s)=\left(\alpha_{1}(t) \beta_{1}(s), \alpha_{1}(t) \beta_{2}(s), \alpha_{2}(t) \beta_{1}(s), \alpha_{2}(t) \beta_{2}(s)\right) .
\end{gathered}
$$

We have

$$
\begin{aligned}
& \frac{\partial f}{\partial t}=\left(\dot{\alpha}_{1}(t) \beta_{1}(s), \dot{\alpha}_{1}(t) \beta_{2}(s), \dot{\alpha}_{2}(t) \beta_{1}(s), \dot{\alpha}_{2}(t) \beta_{2}(s)\right), \\
& \frac{\partial f}{\partial s}=\left(\alpha_{1}(t) \dot{\beta}_{1}(s), \alpha_{1}(t) \dot{\beta}_{2}(s), \alpha_{2}(t) \dot{\beta}_{1}(s), \alpha_{2}(t) \dot{\beta}_{2}(s)\right),
\end{aligned}
$$

where $\dot{\alpha}$ and $\dot{\beta}$ means the first derivative of resp. $\alpha$ and $\beta$.

The coefficients of the pseudo-Riemannian metric $g$ induced on $\operatorname{Imf}$ by the pseudoRiemannian metric of $\mathbb{E}_{2}^{4}$ are

$$
\begin{gathered}
g_{11}=\|\dot{\alpha}\|_{L}^{2}\|\beta\|^{2}, \\
g_{12}=<\alpha, \dot{\alpha}>_{L}<\beta, \dot{\beta}>, \\
g_{22}=\|\alpha\|_{L}^{2}\|\dot{\beta}\|^{2},
\end{gathered}
$$

where \|\|$_{L}$ and $<,>_{L}$ mean the Lorentzian length and inner product on $\mathbb{E}_{1}^{2},\|\|$ and $<,>$ mean the Euclidean length an inner product on $\mathbb{E}^{2}$.

The normal space is spanned by

$$
n_{1}=\left(\dot{\alpha}_{2} \dot{\beta}_{2},-\dot{\alpha}_{2} \dot{\beta}_{1}, \dot{\alpha}_{1} \dot{\beta}_{2},-\dot{\alpha}_{1} \dot{\beta}_{1}\right)
$$

and

$$
n_{2}=\left(\alpha_{2} \beta_{2},-\alpha_{2} \beta_{1}, \alpha_{1} \beta_{2},-\alpha_{1} \beta_{1}\right) .
$$

In the following, we assume that $\alpha$ is a timelike curve or a spacelike curve. Consequently $g_{11} \neq 0$. An orthonormal basis on $\operatorname{Im} \alpha \otimes \beta$ is given by

$$
\begin{gathered}
e_{1}=\frac{1}{\sqrt{\left|g_{11}\right|}} \frac{\partial f}{\partial t}, \\
e_{2}=\frac{1}{\sqrt{\left|g_{11}\left(g_{11} g_{22}-g_{12}^{2}\right)\right|}}\left(g_{11} \frac{\partial f}{\partial s}-g_{12} \frac{\partial f}{\partial t}\right) .
\end{gathered}
$$

The mean curvature vector field $H$ is defined by

$$
H=\frac{1}{2}\left(\varepsilon_{1} h\left(e_{1}, e_{1}\right)+\varepsilon_{2} h\left(e_{2}, e_{2}\right),\right.
$$


where $h$ is the second fundamental form of $\operatorname{Im} f$ and $\varepsilon_{i}=<e_{i}, e_{i}>, \quad i \in\{1,2\}$. Using Beltrami's formula, we also know that

$$
H=-\frac{1}{2} \Delta f
$$

As seen earlier a surface $M$ in $\mathbb{E}_{2}^{4}$ is said to be minimal if its mean curvature vector field vanishes identically. So $\operatorname{Im} f$ is minimal if and only if

$$
<H, n_{i}>=0, \quad i \in\{1,2\} \text {. }
$$

We will determine the tensor product surfaces satisfying the above conditions.

The equation

$$
<H, n_{2}>=0
$$

is equivalent with

$$
<\Delta f, n_{2}>=0 \text {. }
$$

After a straightforward computation we get the Laplacian of $f$, and see that this condition is equal to

$$
<g^{11} \frac{\partial f^{2}}{\partial t^{2}}+g^{22} \frac{\partial f^{2}}{\partial s^{2}}+2 g^{12} \frac{\partial^{2} f}{\partial t \partial s}, n_{2}>=0
$$

Because

we get that

$$
<\frac{\partial f^{2}}{\partial t^{2}}, n_{2}>=<\frac{\partial f^{2}}{\partial s^{2}}, n_{2}>=0
$$

$$
g_{12}<\frac{\partial^{2} f}{\partial t \partial s}, n_{2}>=0
$$

If

$$
g_{12}=0
$$

then either:

(1) $\alpha$ is an orthogonal hyperbola centered at 0 , or

(2) $\beta$ is a circle centered at 0 .

If

$$
<\frac{\partial^{2} f}{\partial t \partial s}, n_{2}>=\left(\alpha_{1} \dot{\alpha}_{2}-\dot{\alpha}_{1} \alpha_{2}\right)\left(\dot{\beta}_{1} \beta_{2}-\beta_{1} \dot{\beta}_{2}\right)=0
$$

then either:

(1) $\alpha$ is a straight line through 0 , or

(2) $\beta$ is a straight line through 0 .

The second condition

$$
<H, n_{1}>=0,
$$

is equivalent with the following equations,

$$
<\Delta f, n_{1}>=0
$$


and

and

$$
<g^{11} \frac{\partial f^{2}}{\partial t^{2}}+g^{22} \frac{\partial f^{2}}{\partial s^{2}}, n_{1}>=0
$$

$$
g_{22}<\frac{\partial f^{2}}{\partial t^{2}}, n_{1}>+g_{11}<\frac{\partial f^{2}}{\partial s^{2}}, n_{1}>=0 .
$$

If we work out this condition, we get that the components of $\alpha$ and $\beta$ must satisfy the following expression,

$$
\begin{gathered}
\left(-\alpha_{1}^{2}+\alpha_{2}^{2}\right)\left(\dot{\beta}_{1}^{2}+\dot{\beta}_{2}^{2}\right)\left(\dot{\alpha}_{1} \ddot{\alpha}_{2}-\ddot{\alpha}_{1} \dot{\alpha}_{2}\right)\left(\beta_{1} \dot{\beta}_{2}-\dot{\beta}_{1} \beta_{2}\right)+ \\
\left(-\dot{\alpha}_{1}^{2}+\dot{\alpha}_{2}^{2}\right)\left(\beta_{1}^{2}+\beta_{2}^{2}\right)\left(\dot{\alpha}_{1} \alpha_{2}-\alpha_{1} \dot{\alpha}_{2}\right)\left(\ddot{\beta}_{1} \dot{\beta}_{2}-\dot{\beta}_{1} \ddot{\beta}_{2}\right)=0 .
\end{gathered}
$$

\section{Case 1.}

Assume $\alpha$ is a straight line through 0 . In this case

$$
\ddot{\alpha}_{1}=\ddot{\alpha}_{2}=\dot{\alpha}_{1} \alpha_{2}-\alpha_{1} \dot{\alpha}_{2}=0,
$$

which means that condition (1) is true for all $\beta$.

The same is true if $\beta$ is a straight line through 0 .

\section{Case 2.}

If $\alpha$ is an orthogonal hyperbola centered at 0 , we can assume that

$$
\alpha(t)=a(\cosh t, \sinh t), \quad a>0 .
$$

( If $\alpha(t)=a(\sinh t, \cosh t)$, we get the same results.)

We calculate $\dot{\alpha}$ and $\ddot{\alpha}$ and look for the curves $\beta$ that satisfy condition (1). The tensor product surface $\alpha \otimes \beta$ is then a minimal surface in $\mathbb{E}_{2}^{4}$. Condition (1) becomes in this case

$$
\left(\dot{\beta}_{1}^{2}+\dot{\beta}_{2}^{2}\right)\left(\beta_{1} \dot{\beta}_{2}-\dot{\beta}_{1} \beta_{2}\right)+\left(\beta_{1}^{2}+\beta_{2}^{2}\right)\left(\dot{\beta}_{1} \ddot{\beta}_{2}-\ddot{\beta}_{1} \dot{\beta}_{2}\right)=0
$$

Because $\beta$ is not a straight line through 0 , its equation in polar coordinates is

$$
\beta(s)=r(s)(\cos s, \sin s) .
$$

The above equation is then equivalent to

$$
r \ddot{r}-3 \dot{r}^{2}-2 r^{2}=0 .
$$

This differential equation can be written as

$$
r \ddot{r}-\dot{r}^{2}-2\left(\dot{r}^{2}+r^{2}\right)=0,
$$


or equivalently

$$
\left(\frac{\dot{r}}{r}\right)^{\prime}-2\left(\left(\frac{\dot{r}}{r}\right)^{2}+1\right)=0 .
$$

Putting

$$
y=\frac{\dot{r}}{r},
$$

we get that

$$
\frac{\dot{y}}{y^{2}+1}=2 \text {. }
$$

The solution of this differential equation is

$$
y=\tan \left(2 s+c_{2}\right) .
$$

After integration, we see that

$$
r(s)=\frac{c_{1}}{\sqrt{\left|\cos \left(2 s+c_{2}\right)\right|}},
$$

where $c_{1}, c_{2} \in \mathbb{R}, c_{1}>0$.

Thus

$$
\beta(s)=\frac{c_{1}}{\sqrt{\left|\cos \left(2 s+c_{2}\right)\right|}}(\cos s, \sin s),
$$

i.e. it is an orthogonal hyperbola centered at 0 .

\section{Case 3.}

If $\beta$ is a circle centered at 0 , assume its equation be given by

$$
\beta(s)=b(\cos s, \sin s), \quad b>0 .
$$

The condition (1) turns out to be

$$
\left(-\alpha_{1}^{2}+\alpha_{2}^{2}\right)\left(\dot{\alpha}_{1} \ddot{\alpha}_{2}-\ddot{\alpha}_{1} \dot{\alpha}_{2}\right)+\left(-\dot{\alpha}_{1}^{2}+\dot{\alpha}_{2}^{2}\right)\left(\alpha_{1} \dot{\alpha}_{2}-\dot{\alpha}_{1} \alpha_{2}\right)=0 .
$$

The equation of an arbitrary curve $\alpha$ in $\mathbb{E}_{1}^{2}$ can be written as

$$
\alpha(t)=\rho(t)(\cosh t, \sinh t),
$$

or

$$
\alpha(t)=\rho(t)(\sinh t, \cosh t) .
$$

These two equations lead to the same differential equation, so we will only consider the first case.

If

$$
\alpha(t)=\rho(t)(\cosh t, \sinh t)
$$

the above condition becomes

$$
\rho \ddot{\rho}-3 \dot{\rho}^{2}+2 \rho^{2}=0 .
$$


This differential equation can also be written as

$$
\left(\frac{\dot{\rho}}{\rho}\right)^{\prime}+2\left(1-\left(\frac{\dot{\rho}}{\rho}\right)^{2}\right)=0 .
$$

Let

$$
y=\frac{\dot{\rho}}{\rho} .
$$

If $y= \pm 1$, we get that

$$
\rho(t)=K e^{ \pm t}, \quad K \in \mathbb{R}_{0} .
$$

Which means that

$$
\alpha(t)=K e^{ \pm t}(\cosh (t), \sinh (t)) .
$$

This case is excluded, because $\alpha$ is a null straight line.

If $y \neq \pm 1$, we get that

$$
\frac{\dot{y}}{1-y^{2}}=-2
$$

There are two cases.

If $|y|<1$, the solution of the differential equation becomes

$$
y=-\tanh (2 t+\mu), \quad \mu \in \mathbb{R} .
$$

After substitution and integration, we see that

$$
\rho(t)=\frac{\gamma}{\sqrt{\cosh (2 t+\mu)}}, \quad \gamma \in \mathbb{R}_{0}
$$

which means that

$$
\alpha(t)=\frac{\gamma}{\sqrt{\cosh (2 t+\mu)}}(\cosh (t), \sinh (t)) .
$$

In this case $\alpha(t)$ is an ellips, moreover when $\mu=0$ we get a circle centered at 0 .

If $|y|>1$, the solution of the differential equation becomes

$$
y=-\operatorname{cotanh}(2 t+\mu), \quad \mu \in \mathbb{R} .
$$

After substitution and integration, we see that

$$
\rho(t)=\frac{\gamma}{\sqrt{\sinh (2 t+\mu)}}, \quad \gamma \in \mathbb{R}_{0}
$$

which means that

$$
\alpha(t)=\frac{\gamma}{\sqrt{\sinh (2 t+\mu)}}(\cosh (t), \sinh (t)) .
$$

In this case $\alpha(t)$ is an orthogonal hyperbola centered at 0 .

Summing up, the following theorem is proved. 
Theorem 2.1. Let $\alpha: \mathbb{R} \rightarrow \mathbb{E}_{1}^{2}$ be a Lorentzian planar curve and $\beta: \mathbb{R} \rightarrow \mathbb{E}^{2}$ be a Euclidean planar curve. Then their tensor product $\alpha \otimes \beta$ is a minimal immersion if and only if either:

(1) either $\alpha$ or $\beta$ is a straight line through 0 ;

(2) both $\alpha$ and $\beta$ are orthogonal hyperbolae centered at 0 ;

(3) $\beta$ is a circle centered at 0 and $\alpha$ is either a circle or an orthogonal hyperbola centered at 0 . 


\section{§3. TOTALLY REAL AND COMPLEX TENSOR PRODUCT SURFACES}

Theorem 3.1. The tensor product $\alpha \otimes \beta$ is a totally real Lorentzian immersion with respect to the pseudo-Hermitian structure $J_{0}$,

$$
J_{0}(u, v, z, w)=(-v, u,-w, z)
$$

on $\mathbb{E}_{2}^{4}$ if and only if

(1) $\alpha$ is an orthogonal hyperbola centered at 0 , or

(2) $\beta$ is a straight line through 0 .

Proof. Assume that $\alpha \otimes \beta$ is totally real. Then

$$
J_{0}\left(\frac{\partial f}{\partial t}\right) \perp \frac{\partial f}{\partial s}
$$

and

$$
J_{0}\left(\frac{\partial f}{\partial s}\right) \perp \frac{\partial f}{\partial t}
$$

With

$$
\begin{aligned}
& \frac{\partial f}{\partial t}=\left(\dot{\alpha}_{1} \beta_{1}, \dot{\alpha}_{1} \beta_{2}, \dot{\alpha}_{2} \beta_{1}, \dot{\alpha}_{2} \beta_{2}\right) \\
& \frac{\partial f}{\partial s}=\left(\alpha_{1} \dot{\beta}_{1}, \alpha_{1} \dot{\beta}_{2}, \alpha_{2} \dot{\beta}_{1}, \alpha_{2} \dot{\beta}_{2}\right)
\end{aligned}
$$

the conditions above become

$$
<\left(-\dot{\alpha}_{1} \beta_{2}, \dot{\alpha}_{1} \beta_{1},-\dot{\alpha}_{2} \beta_{2}, \dot{\alpha}_{2} \beta_{1}\right),\left(\alpha_{1} \dot{\beta}_{1}, \alpha_{1} \dot{\beta}_{2}, \alpha_{2} \dot{\beta}_{1}, \alpha_{2} \dot{\beta}_{2}\right)>=0
$$

and

$$
<\left(-\alpha_{1} \dot{\beta}_{2}, \alpha_{1} \dot{\beta}_{1},-\alpha_{2} \dot{\beta}_{2}, \alpha_{2} \dot{\beta}_{1}\right),\left(\dot{\alpha}_{1} \beta_{1}, \dot{\alpha}_{1} \beta_{2}, \dot{\alpha}_{2} \beta_{1}, \dot{\alpha}_{2} \beta_{2}\right)>=0 .
$$

Both conditions are equivalent with

$$
\left(\alpha_{1} \dot{\alpha}_{1}-\alpha_{2} \dot{\alpha}_{2}\right)\left(\beta_{1} \dot{\beta}_{2}-\dot{\beta}_{1} \beta_{2}\right)=0,
$$

which we had to prove.

Theorem 3.2. The tensor product $\alpha \otimes \beta$ is a complex Lorentzian immersion with respect to the pseudo-Hermitian structure $J_{0}$,

$$
J_{0}(u, v, z, w)=(-v, u,-w, z)
$$

on $\mathbb{E}_{2}^{4}$ if and only if $\alpha$ is a straight line through 0 .

Proof. Since $\alpha \otimes \beta$ is a complex immersion,

$$
J_{0}\left(\frac{\partial f}{\partial t}\right) \perp\left\{n_{1}, n_{2}\right\}
$$


and

$$
J_{0}\left(\frac{\partial f}{\partial s}\right) \perp\left\{n_{1}, n_{2}\right\}
$$

with $\left\{n_{1}, n_{2}\right\}$ a basis for the normal plane.

By straightforward computations, these four equations can be reduced to

$$
\begin{aligned}
& \left(\beta_{1}^{2}+\beta_{2}^{2}\right)\left(\alpha_{1} \dot{\alpha}_{2}-\dot{\alpha}_{1} \alpha_{2}\right)=0 \\
& \left(\dot{\beta}_{1}^{2}+\dot{\beta}_{2}^{2}\right)\left(\alpha_{1} \dot{\alpha}_{2}-\dot{\alpha}_{1} \alpha_{2}\right)=0 .
\end{aligned}
$$

The only solution is that $\alpha$ is a straight line through the origin. 


\section{§4. PSEUDO-MINIMAL TENSOR PRODUCT SURFACES}

We recall that a submanifold $M$ of a pseudo-Riemannian manifold $N$ is said to be pseudo-minimal if the length of its mean curvature vector vanishes identically.

Each minimal submanifold is a pseudo-minimal one. Non-minimal pseudo-minimal submanifolds were studied in particular by R. Rosca (see [R]).

We know that the mean curvature vector field $H$ can be written as

$$
H=a n_{1}+b n_{2},
$$

with $\left\{n_{1}, n_{2}\right\}$ a basis for the normal plane, $a, b$ functions of $t$ and $s$. Using Cramers rule, we can determine $a$ and $b$ from the following equations,

$$
\begin{aligned}
& <H, n_{1}>=a<n_{1}, n_{1}>+b<n_{1}, n_{2}> \\
& <H, n_{2}>=a<n_{1}, n_{2}>+b<n_{2}, n_{2}>.
\end{aligned}
$$

By straightforward computations, we obtain that

$$
\begin{gathered}
<H, H>=\frac{1}{\operatorname{det} g}\left\{<H, n_{1}>^{2}<n_{2}, n_{2}>-2<H, n_{1}><H, n_{2}><n_{1}, n_{2}>\right. \\
\left.+<H, n_{2}>^{2}<n_{1}, n_{1}>\right\},
\end{gathered}
$$

where

$$
\begin{gathered}
<H, n_{1}>=g_{22}<\frac{\partial^{2} f}{\partial t^{2}}, n_{1}>+g_{11}<\frac{\partial^{2} f}{\partial s^{2}}, n_{1}>= \\
<\alpha, \alpha>R^{2}\left(\beta_{1} \dot{\beta}_{2}-\dot{\beta}_{1} \beta_{2}\right)\left(\ddot{\alpha}_{1} \dot{\alpha}_{2}-\dot{\alpha}_{1} \ddot{\alpha}_{2}\right)+<\dot{\alpha}, \dot{\alpha}>r^{2}\left(\alpha_{1} \dot{\alpha}_{2}-\dot{\alpha}_{1} \alpha_{2}\right)\left(\ddot{\beta}_{1} \dot{\beta}_{2}-\dot{\beta}_{1} \ddot{\beta}_{2}\right)
\end{gathered}
$$

with

$$
\begin{aligned}
& r^{2}=\beta_{1}^{2}+\beta_{2}^{2}, \\
& R^{2}=\dot{\beta}_{1}^{2}+\dot{\beta}_{2}^{2},
\end{aligned}
$$

and

$$
<H, n_{2}>=-2<\alpha, \dot{\alpha}>\left(\beta_{1} \dot{\beta}_{1}+\beta_{2} \dot{\beta}_{2}\right)\left(\dot{\alpha}_{1} \dot{\alpha}_{2}-\alpha_{1} \dot{\alpha}_{2}\right)\left(\dot{\beta}_{1} \beta_{2}-\beta_{1} \dot{\beta}_{2}\right) \text {. }
$$

Using Theorem 2.1, we can assume that neither $\alpha$ nor $\beta$ is a straight line through 0 . Thus, we put

$$
\alpha(t)=\rho(\cosh t, \sinh t)
$$

and

$$
\beta(s)=r(\cos s, \sin s) .
$$

$(\alpha(t)=\rho(\sinh t, \cosh t)$ givesusthesamedifferentialequation. $)$

The equation $\langle H, H\rangle=0$ then leads to

$$
\begin{gathered}
{\left[\left(r^{2}+\dot{r}^{2}\right)\left(\rho \ddot{\rho}-\dot{\rho}^{2}\right)-2\left(r^{2}+\dot{r}^{2}\right)\left(\dot{\rho}^{2}-\rho^{2}\right)+\left(\dot{\rho}^{2}-\rho^{2}\right)\left(r \ddot{r}-\dot{r}^{2}\right)\right]^{2}} \\
+4 \dot{r}^{2} \dot{\rho}^{2}\left[\left(\rho \ddot{\rho}-\dot{\rho}^{2}\right)\left(r^{2}+\dot{r}^{2}\right)-\left(\dot{\rho}^{2}-\rho^{2}\right)\left(r^{2}+\dot{r}^{2}\right)+\left(\dot{\rho}^{2}-\rho^{2}\right)\left(r \ddot{r}-\dot{r}^{2}\right)\right]=0 .
\end{gathered}
$$


If $\dot{\rho}=0$, i.e. $\alpha$ is an orthogonal hyperbola centered at 0 , then the condition above becomes

$$
2 r^{2}+3 \dot{r}^{2}-r \ddot{r}=0,
$$

solving this differential equation gives us a minimal surface.

If $\dot{r}=0$, i.e. $\beta$ is a circle centered at 0 , the condition turns out to be,

$$
\rho \ddot{\rho}-3 \dot{\rho}^{2}+2 \rho^{2}=0,
$$

which gives us also minimal surfaces.

We can conclude that, if either $\dot{\rho}=0$ or $\dot{r}=0$, there do not exist non-minimal pseudo-minimal tensor product surfaces $\alpha \otimes \beta$.

If $\dot{\rho} \neq 0$ and $\dot{r} \neq 0$, we denote

$$
\delta=\frac{r}{\dot{r}}
$$

and

$$
\gamma=\frac{\rho}{\dot{\rho}} .
$$

The equation (4.1) of a pseudo-minimal surface becomes

$$
\begin{gathered}
{\left[\left(\delta^{2}+1\right) \dot{\gamma}+2\left(\delta^{2}+1\right)\left(1-\gamma^{2}\right)+\left(1-\gamma^{2}\right) \dot{\delta}\right]^{2}} \\
-4\left[\left(\delta^{2}+1\right) \dot{\gamma}+\left(1-\gamma^{2}\right) \dot{\delta}+\left(1-\gamma^{2}\right)\left(1+\delta^{2}\right)\right]=0 .
\end{gathered}
$$

We distinguish two cases.

a) $\gamma^{2}=1$.

It's easy to see that this is a solution of equation (4.2). In this case

$$
\frac{\rho}{\dot{\rho}}= \pm 1
$$

By integrating, we see that

$$
\rho=K e^{ \pm t}
$$

with $K \in \mathbb{R}_{0}$. The equation of $\alpha$ is given by

$$
\alpha(t)=K e^{ \pm t}(\cosh t, \sinh t) \quad, \quad K \in \mathbb{R}_{0} .
$$

This case is excluded, because $\alpha$ is a null straight line.

b) $\gamma^{2} \neq 1$.

We introduce the following notations:

$$
F(t, s)=\frac{\dot{\gamma}}{1-\gamma^{2}}+\frac{\dot{\delta}}{1+\delta^{2}}+2
$$

and

$$
P(t, s)=\left(1+\delta^{2}\right)\left(1-\gamma^{2}\right) .
$$


The equation (4.2) reduces to

Then

$$
F^{2}-\frac{4}{P} F+\frac{4}{P}=0
$$

$$
F=\frac{2(1 \pm \sqrt{1-P})}{P} .
$$

From equation (4.3) we see immediatly that $\frac{\partial F}{\partial t}$ is a function of $t$ and $\frac{\partial F}{\partial s}$ is a function of $s$. We can also calculate $\frac{\partial F}{\partial t}$ and $\frac{\partial F}{\partial s}$ using equation (4.5). From (4.4) we get that

and

$$
\frac{\partial P}{\partial t}=-2 \gamma \dot{\gamma}\left(1+\delta^{2}\right)
$$

$$
\frac{\partial P}{\partial s}=2 \delta \dot{\delta}\left(1-\gamma^{2}\right)
$$

So using (4.5) and after some differentiation, we have that

and

$$
\frac{\partial F}{\partial t}=\frac{2 \gamma \dot{\gamma}}{1-\gamma^{2}}\left(\frac{2}{p}(1 \pm \sqrt{1-p}) \pm \frac{1}{\sqrt{1-p}}\right)
$$

Now it is easy to see that

$$
\frac{\partial F}{\partial s}=\frac{-2 \delta \dot{\delta}}{1+\delta^{2}}\left(\frac{2}{P}(1 \pm \sqrt{1-P}) \pm \frac{1}{\sqrt{1-P}}\right) .
$$

$$
\frac{2}{P}(1 \pm \sqrt{1-P}) \pm \frac{1}{\sqrt{1-P}}=c
$$

with $c \in \mathbb{R}$. If we take the partial derivative with respect to $t$ we see that

This leads to

$$
\left(\frac{2 \gamma \dot{\gamma}}{1-\gamma^{2}}\right) c \pm \frac{1}{2(1-P)^{\frac{3}{2}}} \frac{\partial P}{\partial t}=0
$$

$$
\pm \frac{P}{2(1-P)^{3 / 2}}=c \text {. }
$$

The partial derivative with respect to $t$ of this last equation gives

The partial derivative with respect to $s$ gives

$$
\frac{\partial P}{\partial t} \sqrt{1-p}\left(1+\frac{P}{2}\right)=0 .
$$

$$
\frac{\partial P}{\partial s} \sqrt{1-p}\left(1+\frac{P}{2}\right)=0 .
$$

The solution of these equations is that $P=$ constant . It's easy to see from

$$
P(t, s)=\left(1+\delta^{2}\right)\left(1-\gamma^{2}\right)=\text { constant }
$$

that both $\delta$ and $\gamma$ are constants. In this case

where $a, b, k, l \in \mathbb{R}_{0}, a>0$ and $l^{2} \neq 1$.

$$
r(s)=a e^{b s} \text { and } \rho(t)=k e^{l t},
$$

The pseudo-minimality condition is then equivalent to $l^{2}-b^{2}=1$.

A classification of pseudo-minimal tensor product surfaces is thus given by the following theorem. 
Theorem 4.1. The tensor product $\alpha \otimes \beta$ is a non-minimal pseudo-minimal immersion if and only if

$$
\alpha(t)=k e^{ \pm \sqrt{1+b^{2}} t}(\cosh t, \sinh t) \quad, \quad k, b \in \mathbb{R}_{0},
$$

and

$$
\beta(s)=a e^{b s}(\cos s, \sin s) \quad, \quad a>0, \quad b \in \mathbb{R}_{0}
$$

i.e. $\alpha$ is a hyperbolic spiral and $\beta$ is a logarithmic spiral, respectively. 


\section{§5. PSEUDO-UMBILICAL TENSOR PRODUCT SURFACES}

An isometric immersion $f$ is said to be pseudo-umbilical if the shape operator with respect to $H$ is proportional to the identity.

An equivalent condition is the following:

$$
<h(X, Y), H\rangle=\lambda\langle X, Y\rangle \quad, \quad X, Y \in \Gamma(T M),
$$

where $\lambda=<H, H>$.

It is easily seen that each minimal submanifold is pseudo-umbilical.

In this section we determine pseudo-umbilical tensor products of an orthogonal hyperbola centered at the origin in $\mathbb{E}_{1}^{2}$ and an arbitrary curve in $\mathbb{E}^{2}$.

Let $\alpha$ be an orthogonal hyperbola centered at 0 , say

$$
\alpha(t)=a(\cosh t, \sinh t),
$$

(the same is true if $\alpha(t)=a(\sinh t, \cosh t)$ )

and let $\beta$ be an arbitrary Euclidean planar curve. The Laplacian of $f$ is given by

$$
\Delta f=\frac{1}{a^{2}}\left[\frac{1}{\|\beta\|^{2}} \frac{\partial^{2} f}{\partial t^{2}}-\left(\frac{<\beta, \dot{\beta}>}{\|\beta\|^{2}\|\dot{\beta}\|^{2}}-\frac{<\dot{\beta}, \ddot{\beta}>}{\|\dot{\beta}\|^{4}}\right) \frac{\partial f}{\partial s}-\frac{1}{\|\dot{\beta}\|^{2}} \frac{\partial^{2} f}{\partial s^{2}}\right] .
$$

If we put in condition (5.1) respectively

$$
X=Y=\frac{\partial f}{\partial t}
$$

and

we get that

$$
X=Y=\frac{\partial f}{\partial s}
$$

$$
\begin{aligned}
& <\frac{\partial^{2} f}{\partial t^{2}}, H>=\lambda g_{11}, \\
& <\frac{\partial^{2} f}{\partial s^{2}}, H>=\lambda g_{22} .
\end{aligned}
$$

If we use $H=-\frac{1}{2} \Delta f$ and eliminate $\lambda$ from these equations, we obtain after long computations that

$$
\|\dot{\beta}\|^{2}-\frac{<\beta, \dot{\beta}>^{2}}{\|\beta\|^{2}}-\frac{\|\beta\|^{2}\|\ddot{\beta}\|^{2}}{\|\dot{\beta}\|^{2}}+<\dot{\beta}, \ddot{\beta}>^{2} \frac{\|\beta\|^{2}}{\|\dot{\beta}\|^{4}}=0 .
$$

If $\beta$ is not a straight line containing 0 , consider its equation in polar coordinates

$$
\beta(s)=r(s)(\cos s, \sin s) .
$$

The equation then becomes:

$$
\left(r \ddot{r}-\dot{r}^{2}\right)\left(r \ddot{r}-3 \dot{r}^{2}-2 r^{2}\right)=0 .
$$


The equation

$$
r \ddot{r}-3 \dot{r}^{2}-2 r^{2}=0
$$

gives us the minimal surfaces, so we only consider the following equation

$$
r \ddot{r}-\dot{r}^{2}=0 .
$$

The solution is

$$
r=k e^{b s}, \quad k, b \in \mathbb{R}_{0}
$$

( $b$ equal to 0 gives a minimal surface).

Summarizing, we obtain the following result.

Theorem 5.1. The tensor product $\alpha \otimes \beta$ of an orthogonal hyperbola $\alpha$ centered at the origin in $\mathbb{E}_{1}^{2}$ and a Euclidean planar curve $\beta$ is a non-minimal pseudo-umbilical surface in $\mathbb{E}_{2}^{4}$ if and only if $\beta$ is a logaritmic spiral in $\mathbb{E}^{2}$, i.e.

$$
\beta(s)=k e^{b s}(\cos s, \sin s), \quad k, b \in \mathbb{R}_{0} .
$$

In this last section we determine pseudo-umbilical tensor products of an arbitrary curve $\alpha$ in $\mathbb{E}_{1}^{2}$ and a circle $\beta$ centered at the origin in $\mathbb{E}^{2}$.

Let

$$
\beta(s)=a(\cos s, \sin s)
$$

and let $\alpha$ be a curve in $\mathbb{E}_{1}^{2}$, we have that

$$
H=\frac{-1}{2 a^{2}}\left[\left(\frac{<\alpha, \dot{\alpha}>_{L}}{\|\alpha\|_{L}^{2}\|\dot{\alpha}\|_{L}^{2}}-\frac{<\dot{\alpha}, \ddot{\alpha}>_{L}}{\|\dot{\alpha}\|_{L}^{4}}\right) \frac{\partial f}{\partial t}+\frac{1}{\|\dot{\alpha}\|_{L}^{2}} \frac{\partial^{2} f}{\partial t^{2}}+\frac{1}{\|\alpha\|_{L}^{2}} \frac{\partial^{2} f}{\partial s^{2}}\right] .
$$

The condition for pseudo-umbilicality turns out to be

$$
\|\dot{\alpha}\|_{L}^{2}-\frac{<\alpha, \dot{\alpha}>_{L}^{2}}{\|\alpha\|_{L}^{2}}+\frac{<\dot{\alpha}, \ddot{\alpha}>_{L}^{2}\|\alpha\|_{L}^{2}}{\|\dot{\alpha}\|_{L}^{4}}-\frac{\|\alpha\|_{L}^{2}\|\ddot{\alpha}\|_{L}^{2}}{\|\dot{\alpha}\|_{L}^{2}}=0 .
$$

After a lot of computations and knowing that $\alpha$ is not a straight line through the origin, so

$$
\alpha(t)=r(t)(\cosh t, \sinh t),
$$

(the same is true when $\alpha(t)=r(t)(\sinh t, \cosh t)$ )

we get the following equation to solve:

$$
\left(r \ddot{r}-\dot{r}^{2}\right)\left(r \ddot{r}-3 \dot{r}^{2}+2 r^{2}\right)=0 .
$$

The second term of the equation equal to 0 , gives minimal surfaces, so we only consider the first term equal to 0 :

$$
r \ddot{r}-\dot{r}^{2}=0 .
$$

The solution is

$$
r(t)=k e^{b t}, \quad k, b \in \mathbb{R}_{0} .
$$

( $b \neq \pm 1$ otherwise we get a null straight line)

We can conclude: 
Theorem 5.2. The tensor product $\alpha \otimes \beta$ of an arbitrary curve $\alpha$ in $\mathbb{E}_{1}^{2}$ and a circle $\beta$ centered at the origin in $\mathbb{E}^{2}$ is a non-minimal pseudo-umbilical surface in $\mathbb{E}_{2}^{4}$ if and only if $\alpha$ is a hyperbolic spiral, i.e.

$$
\alpha(t)=k e^{b t}(\cosh t, \sinh t), \quad k, b \in \mathbb{R}_{0} \backslash\{-1,1\}
$$




\section{PICTURES}

Figure 1 : The Möbius strip

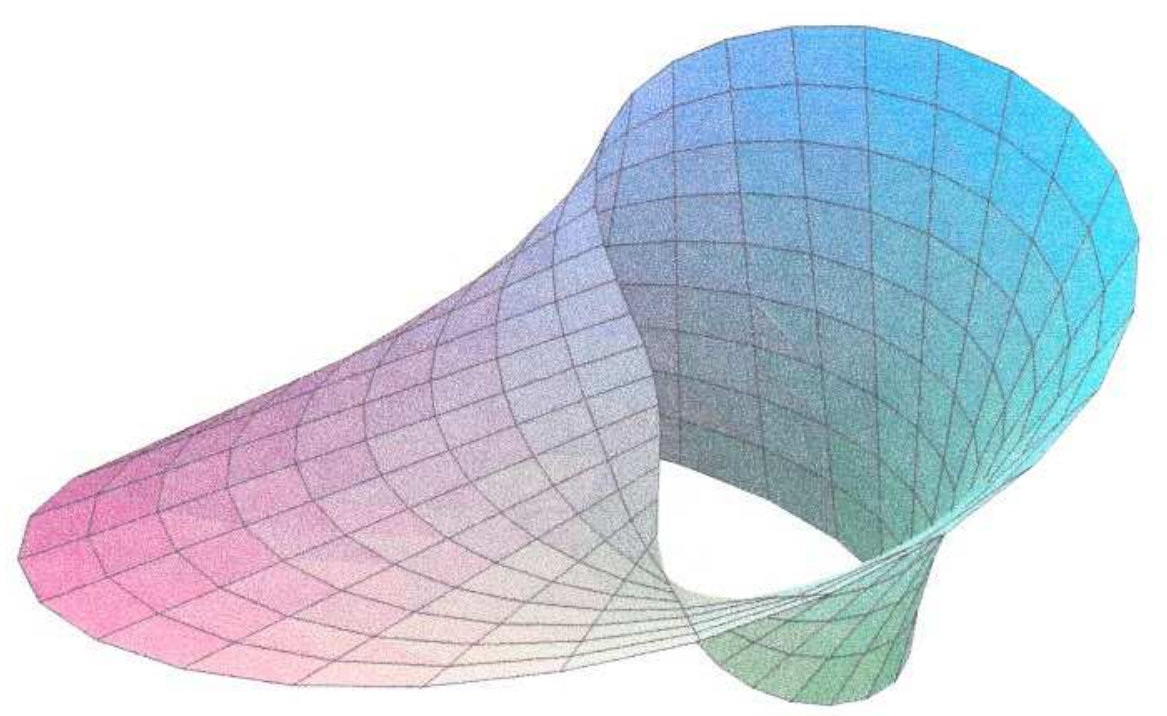

$X(s, t)=(\cos s, \sin s, 0)+t(\cos (s / 2) \cos s, \cos (s / 2) \sin s, \sin (s / 2))$ 
Figure 2 : The one-sheeted hyperboloid

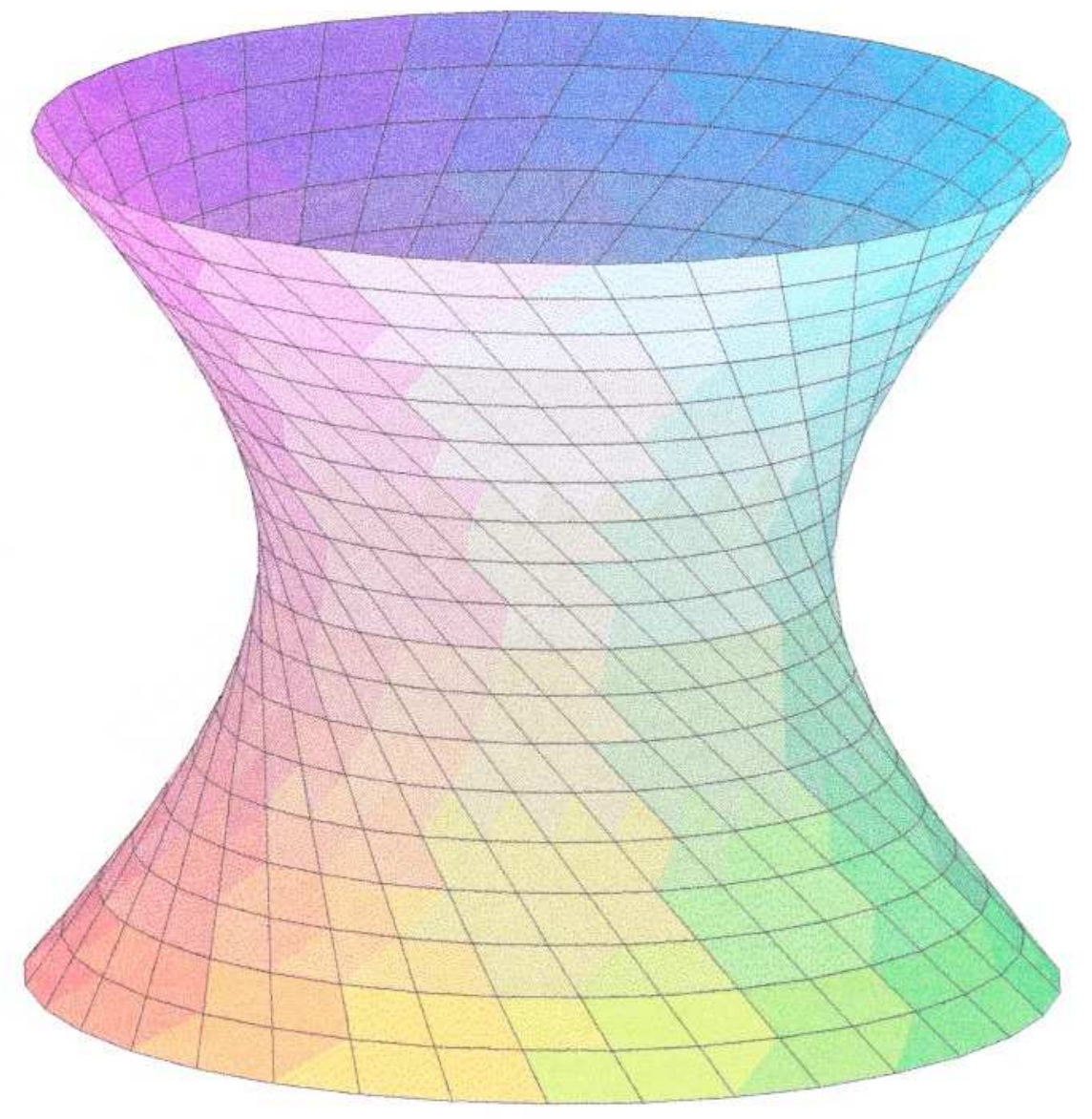

$X(s, t)=(a \cos s, b \sin s, 0)+t(a \sin s,-b \cos s, c), \quad a, b, c \in \mathbb{R}_{0}$ 
Figure 3a : The hyperbolic paraboloid

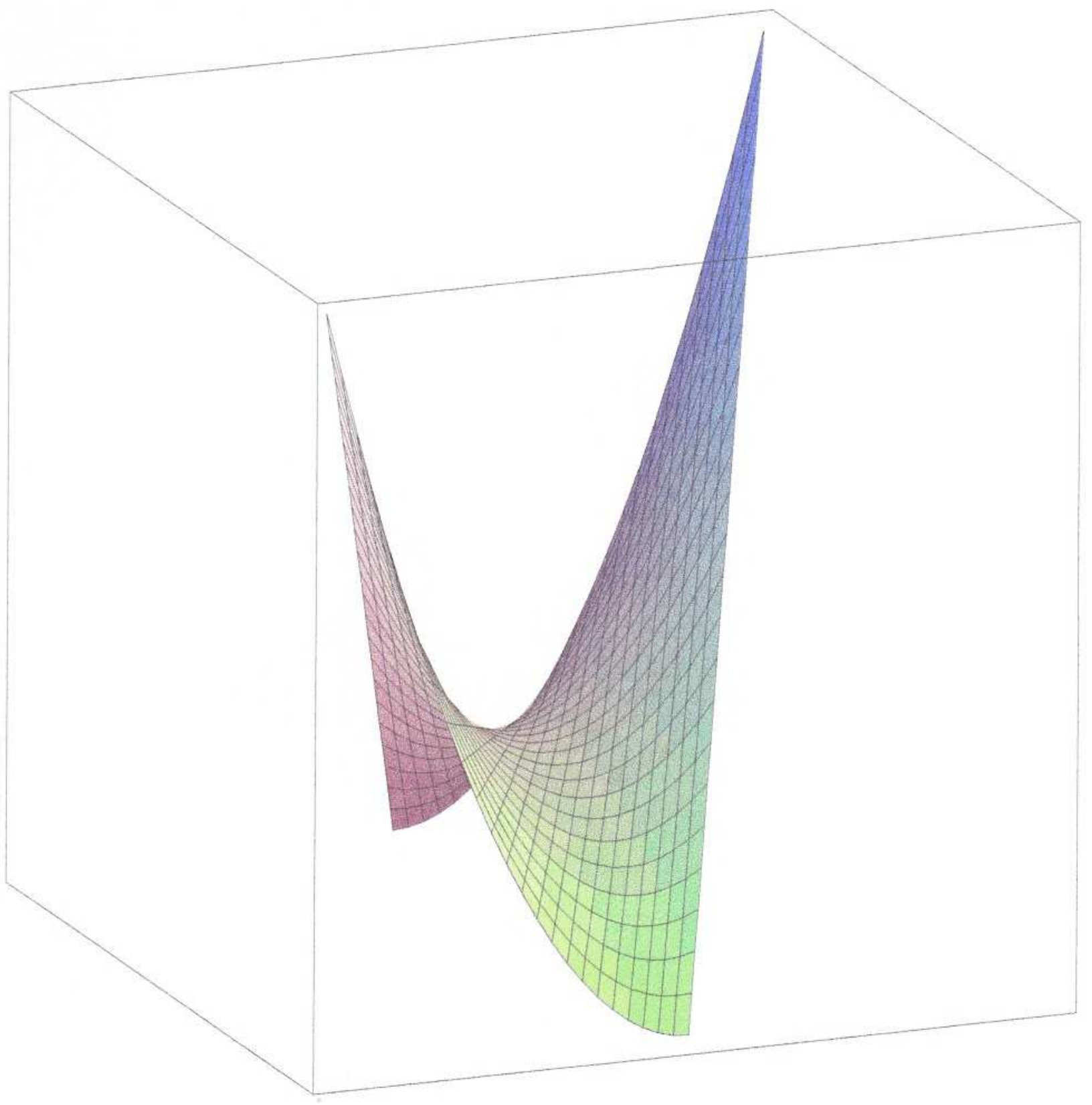

$$
X(s, t)=\left(a s, 0, s^{2}\right)+t(a, b, 2 s), \quad a, b \in \mathbb{R}_{0}
$$


Figure 3b : The hyperbolic paraboloid

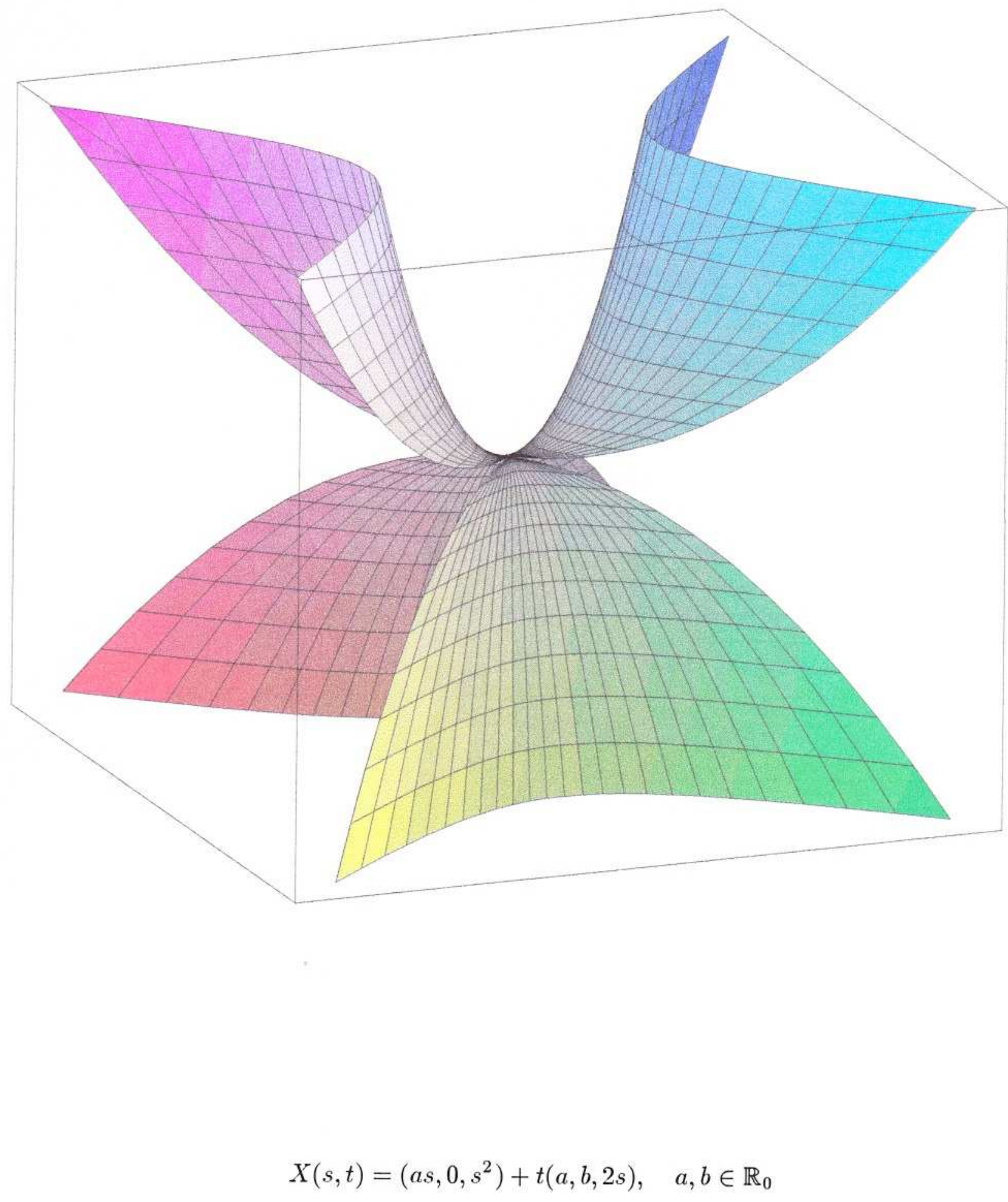


Figure 4 : The helicoid

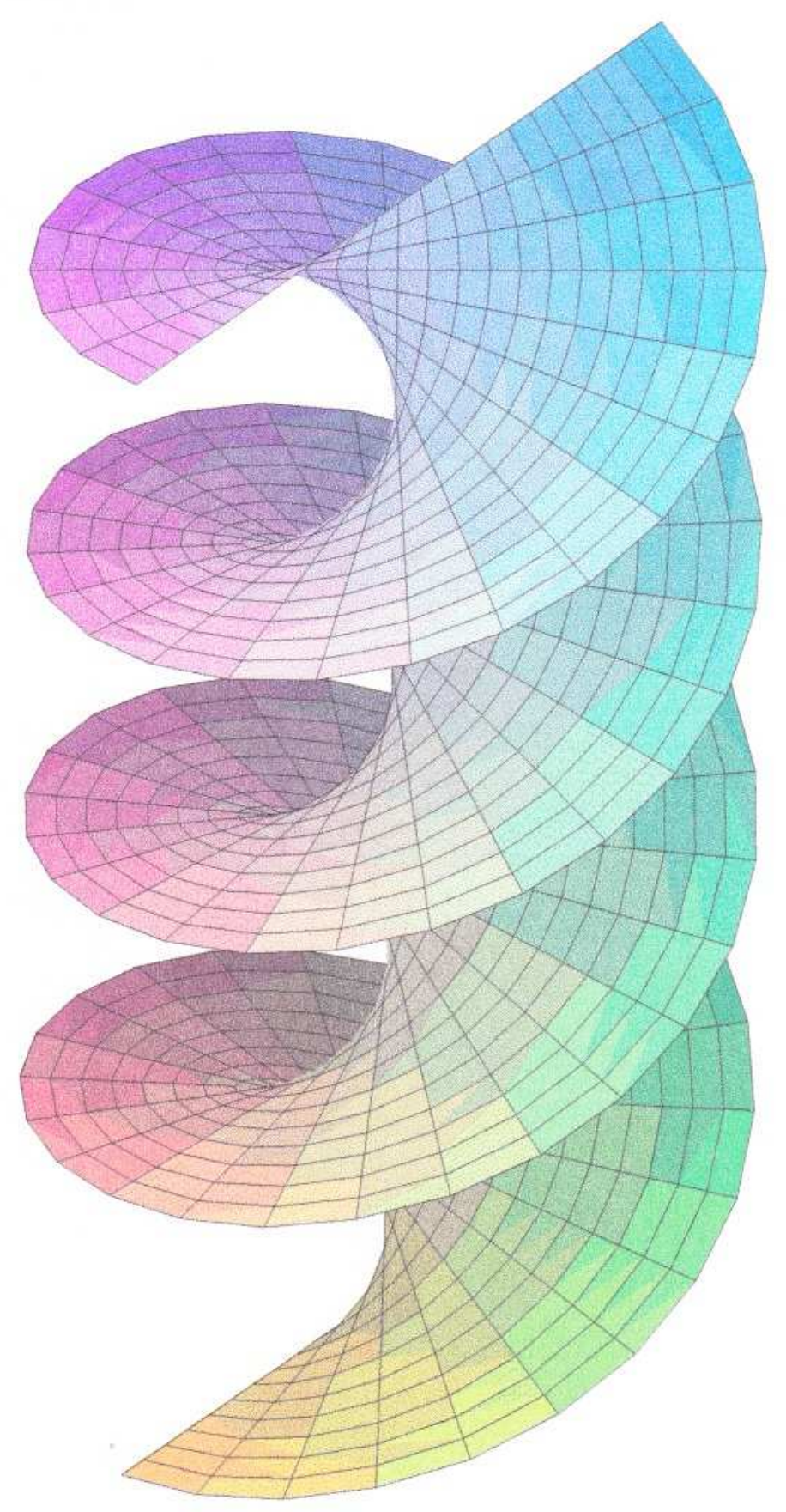

$$
X(s, t)=(0,0, b s)+t(\cos s, \sin s, 0), \quad b \neq 0
$$


Figure 5 : The helicoid of second kind

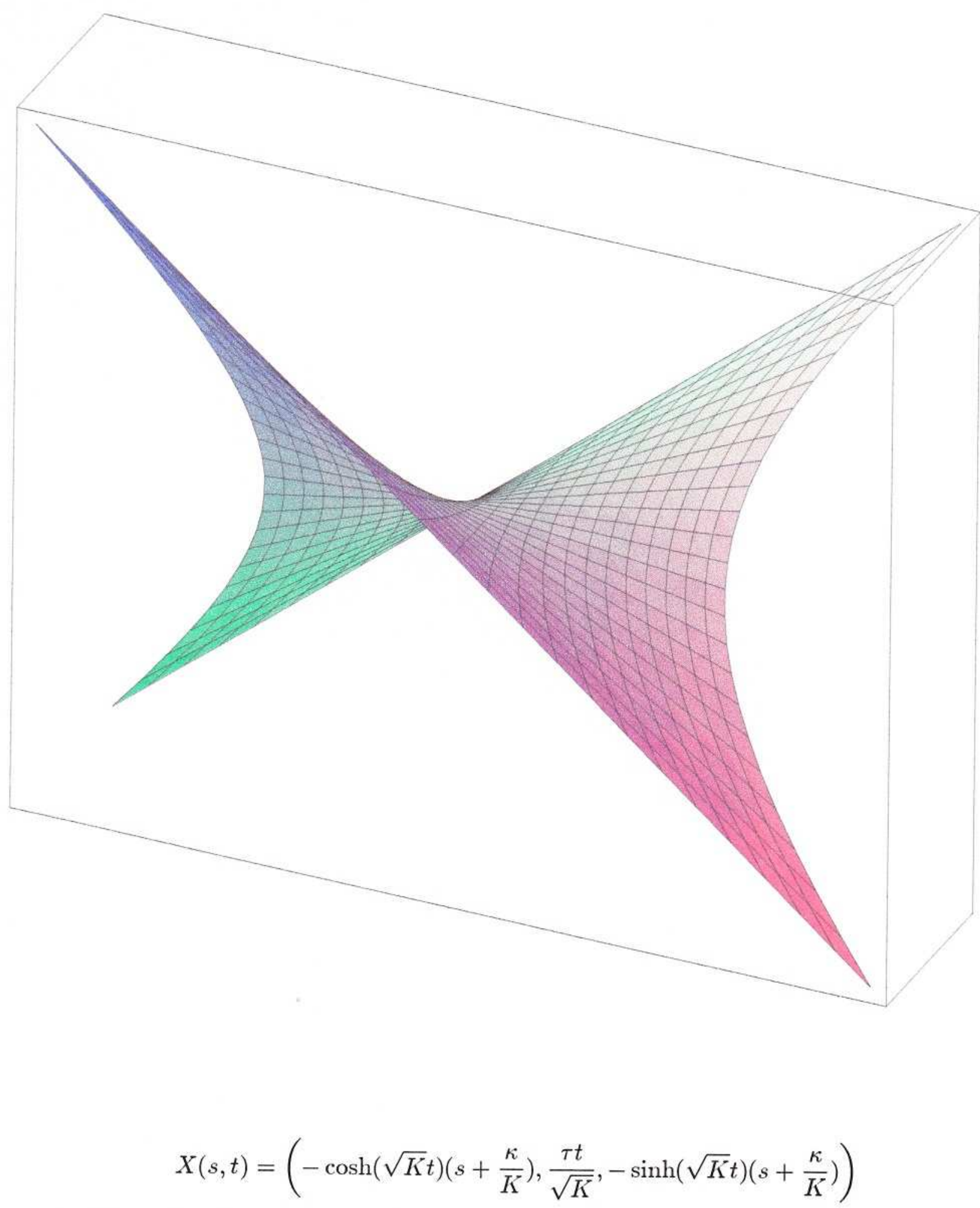

with $K=\kappa^{2}-\tau^{2}, \quad$ where $\kappa$ is the curvature and $\tau$ is the torsion of $\alpha$ with $|\kappa|>|\tau|>0$ 
Figure 6 : The helicoid of third kind

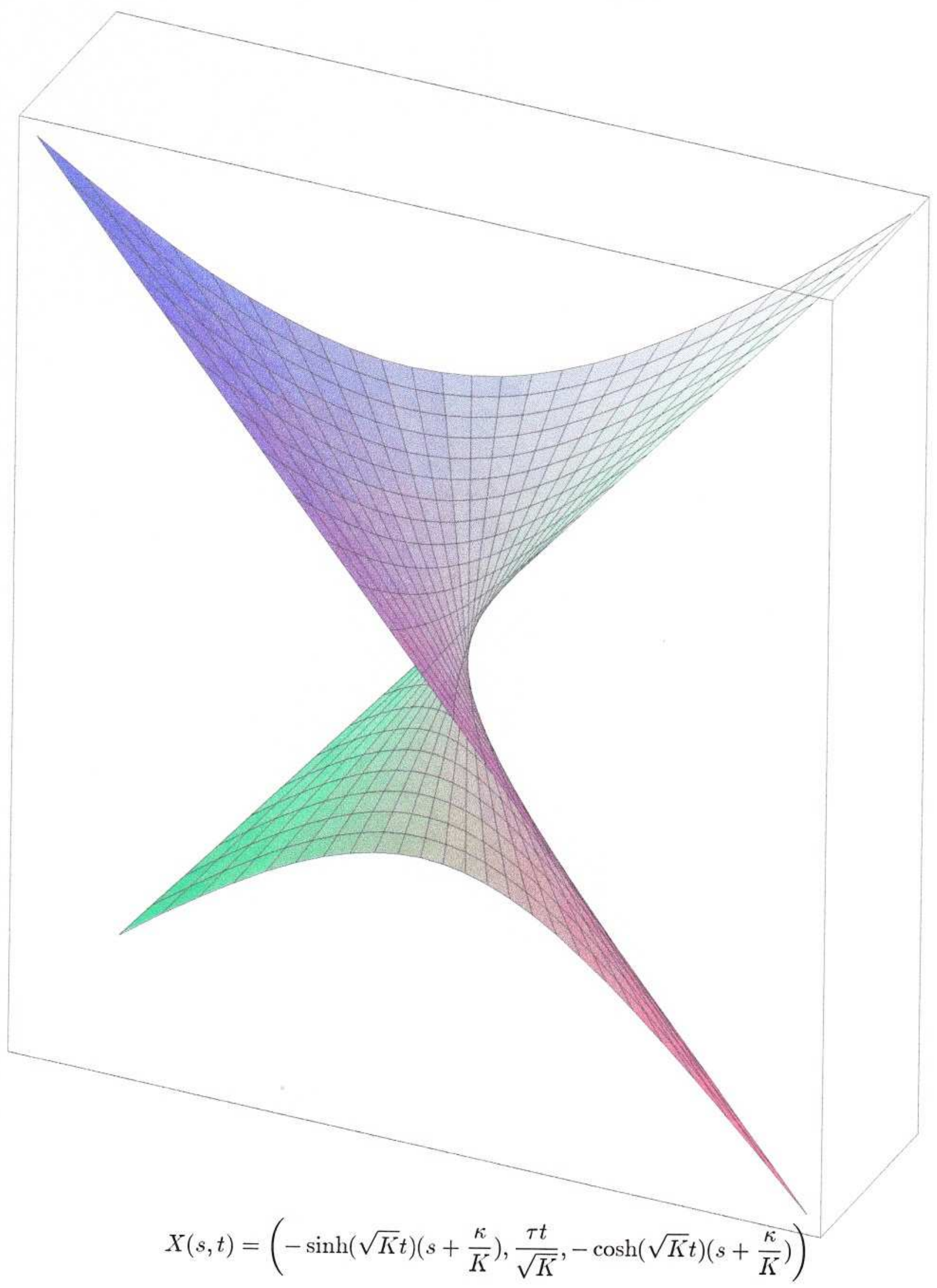

with $K=\kappa^{2}+\tau^{2}$, where $\kappa$ is the curvature and $\tau$ is the torsion of $\alpha$ 
Figure 7 : The conjugate of Enneper's surface of second kind

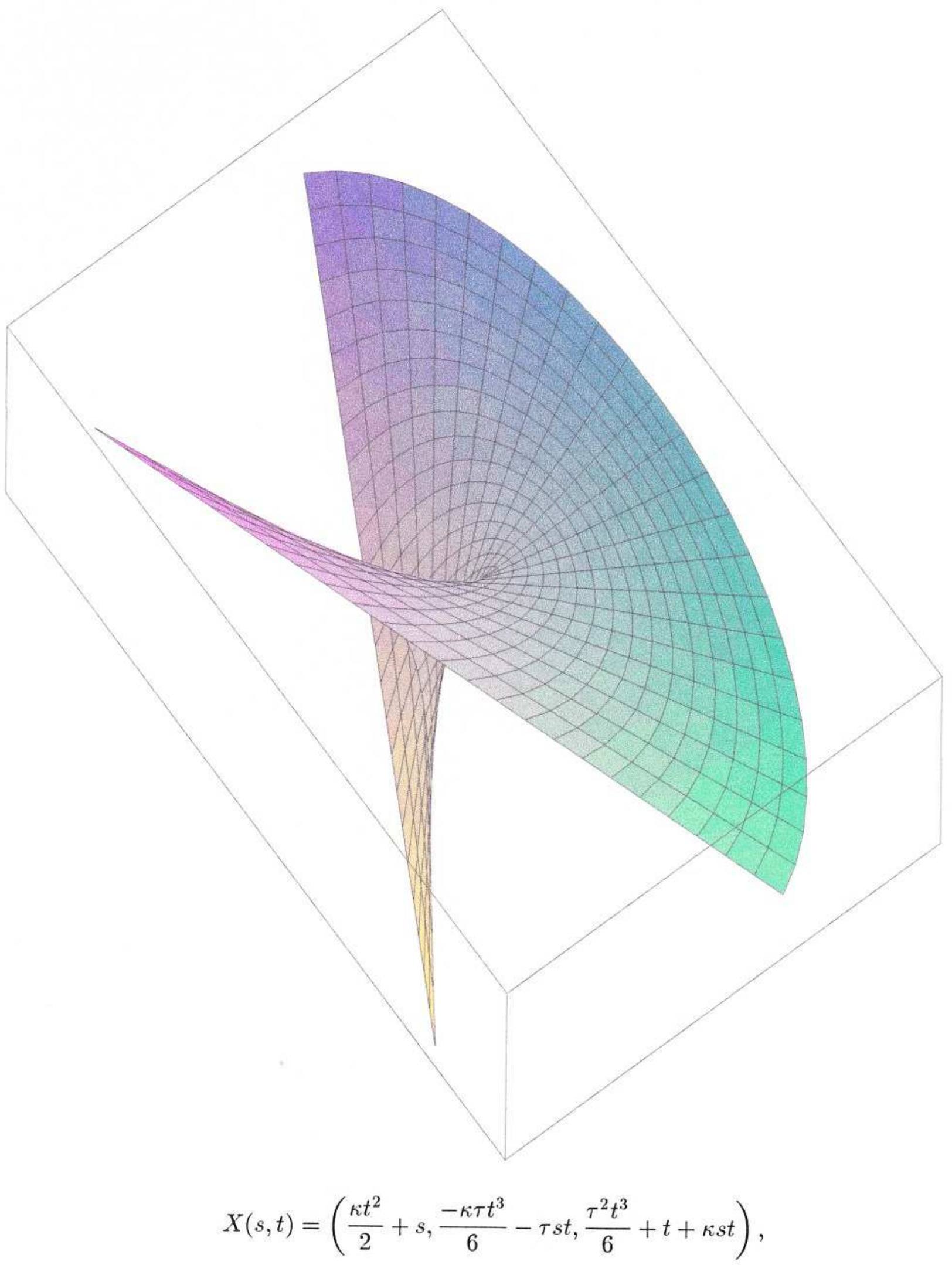

where $\kappa$ is the curvature and $\tau$ is the torsion of $\alpha$ with $|\tau|=|\kappa|>0$ 
Figure 8 : The surface of Scherk

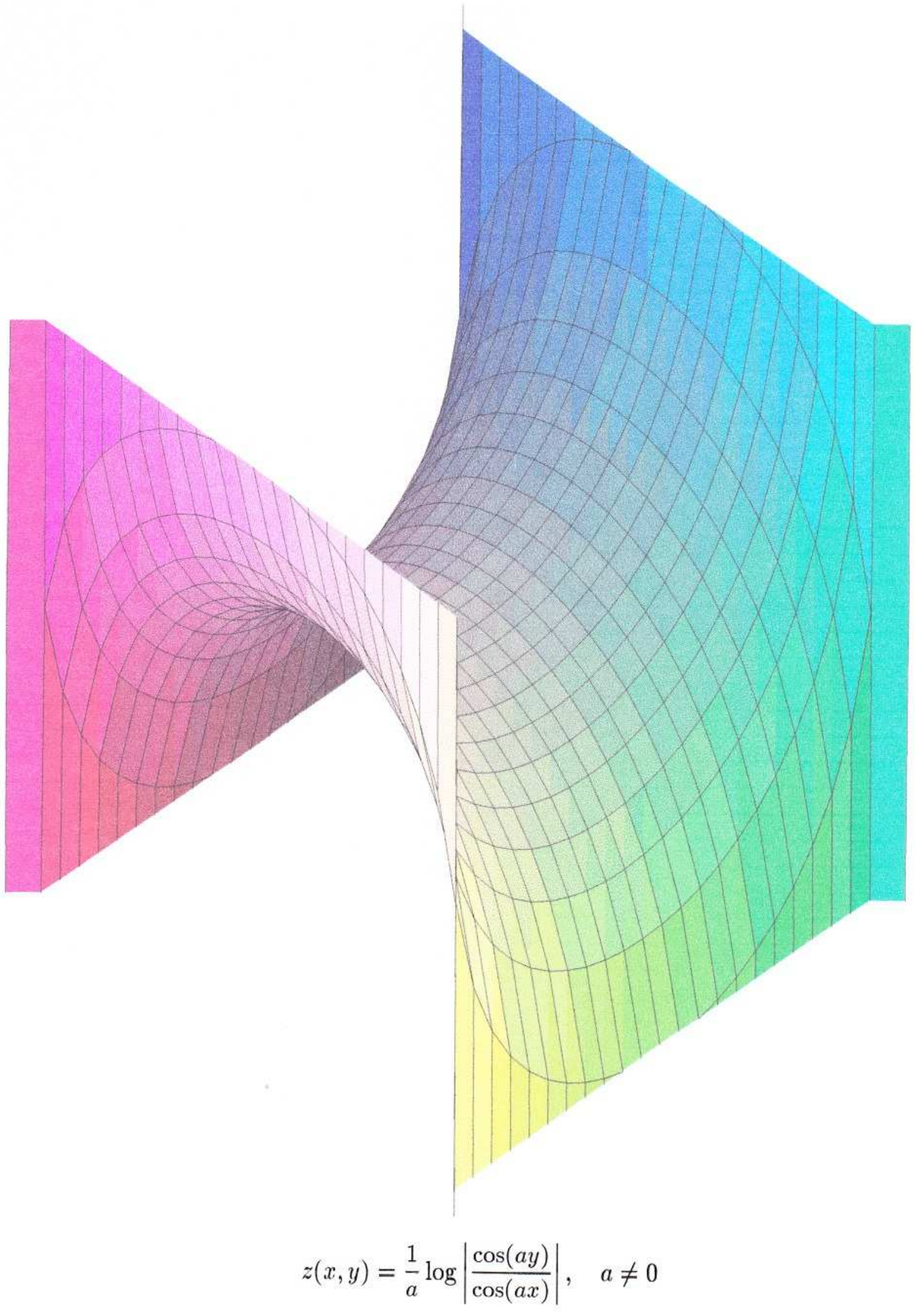


Figure 9 : The surface of Scherk of second kind

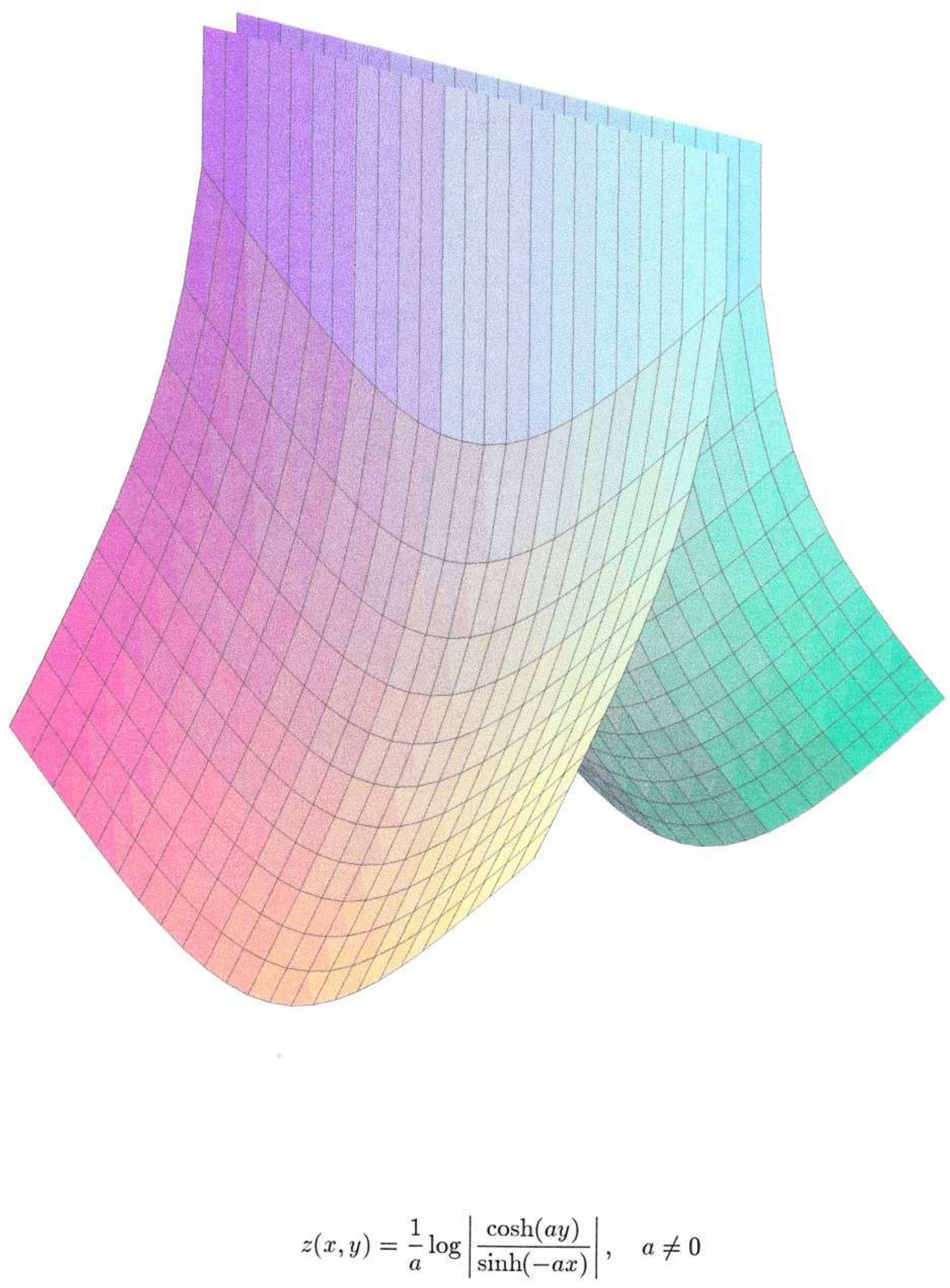


Figure 10 : The surface of Scherk of third kind

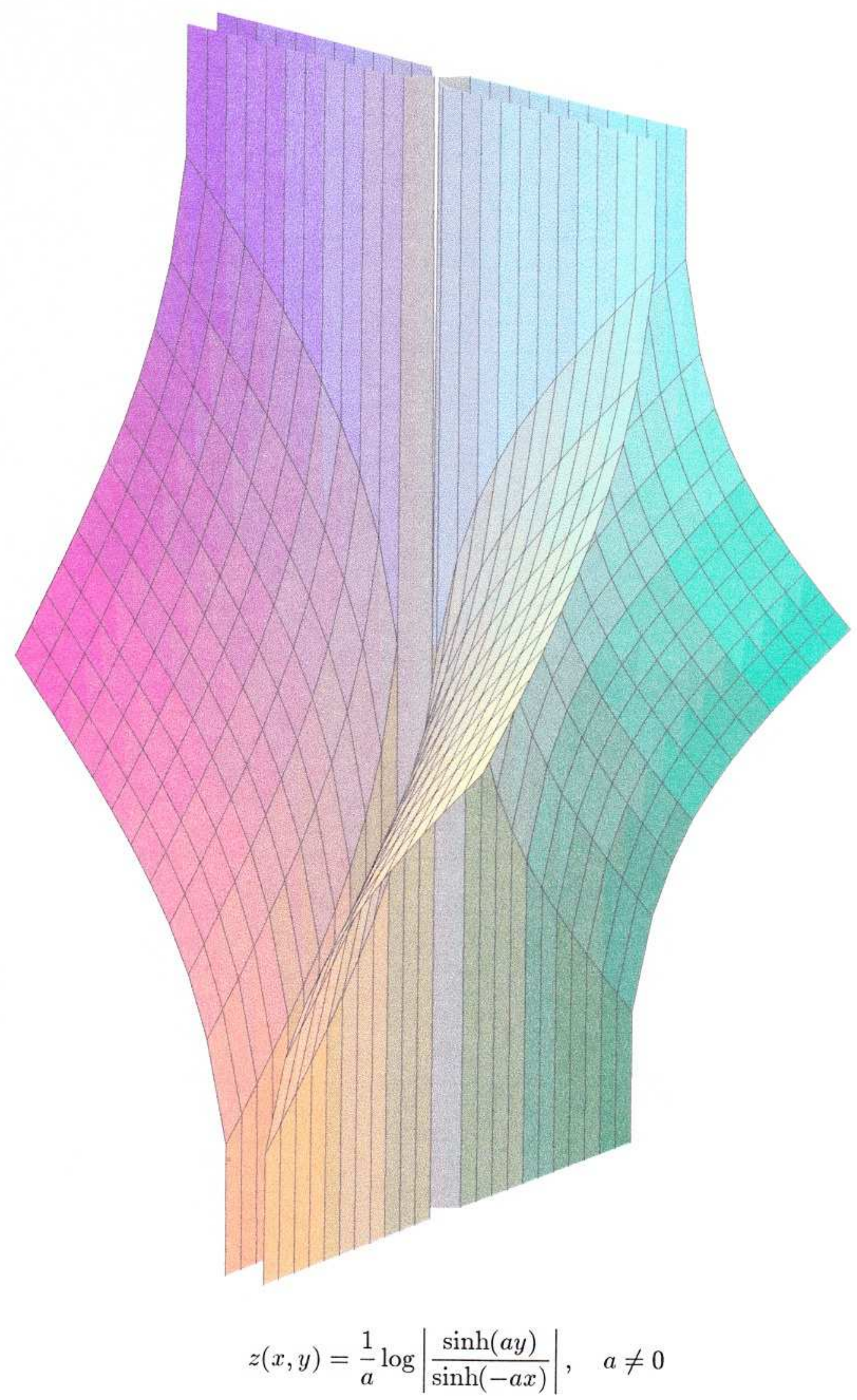


Figure 11 : The surface of Scherk of fourth kind

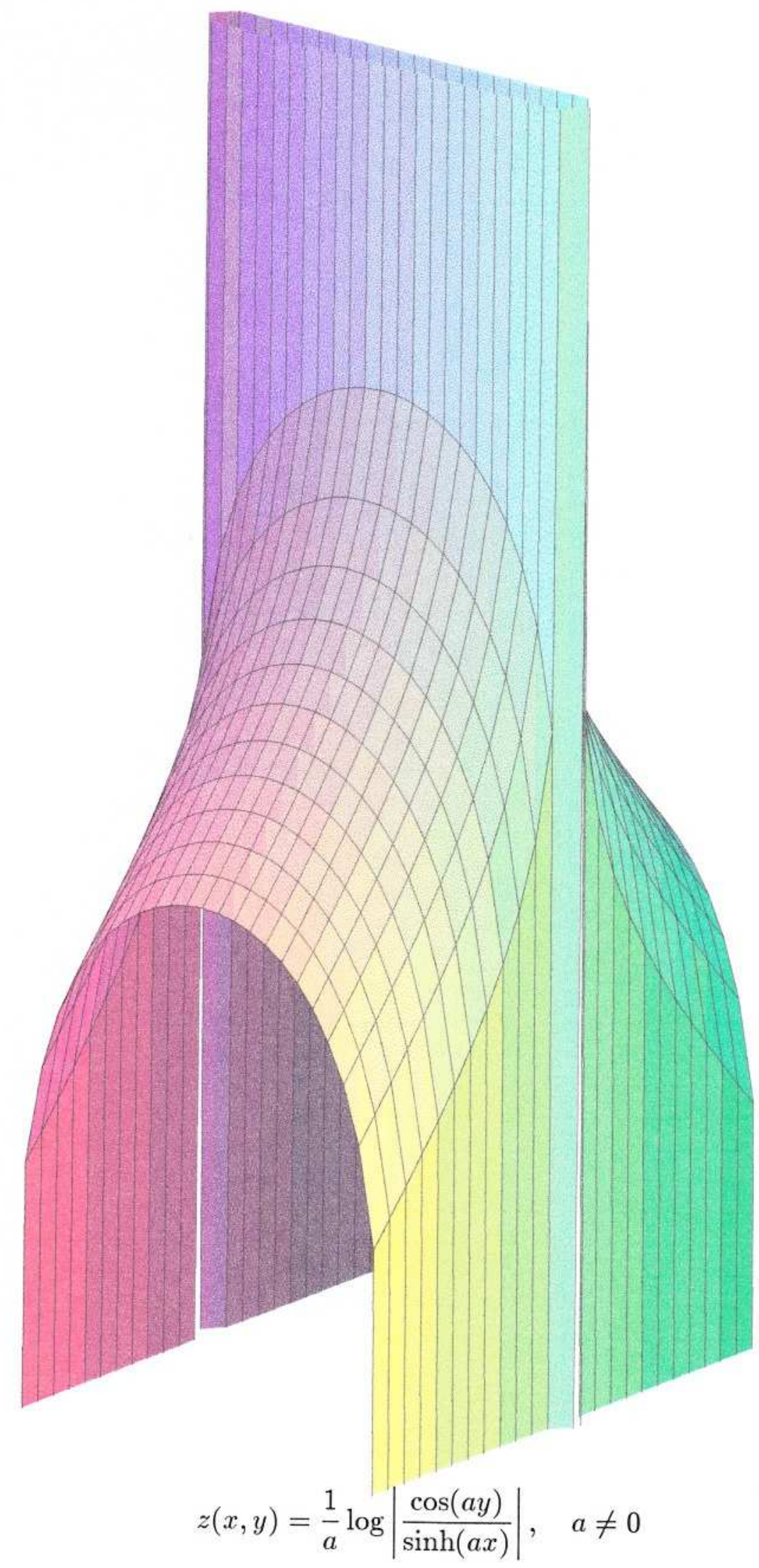


Figure 12 : The surface of Scherk of fifth kind

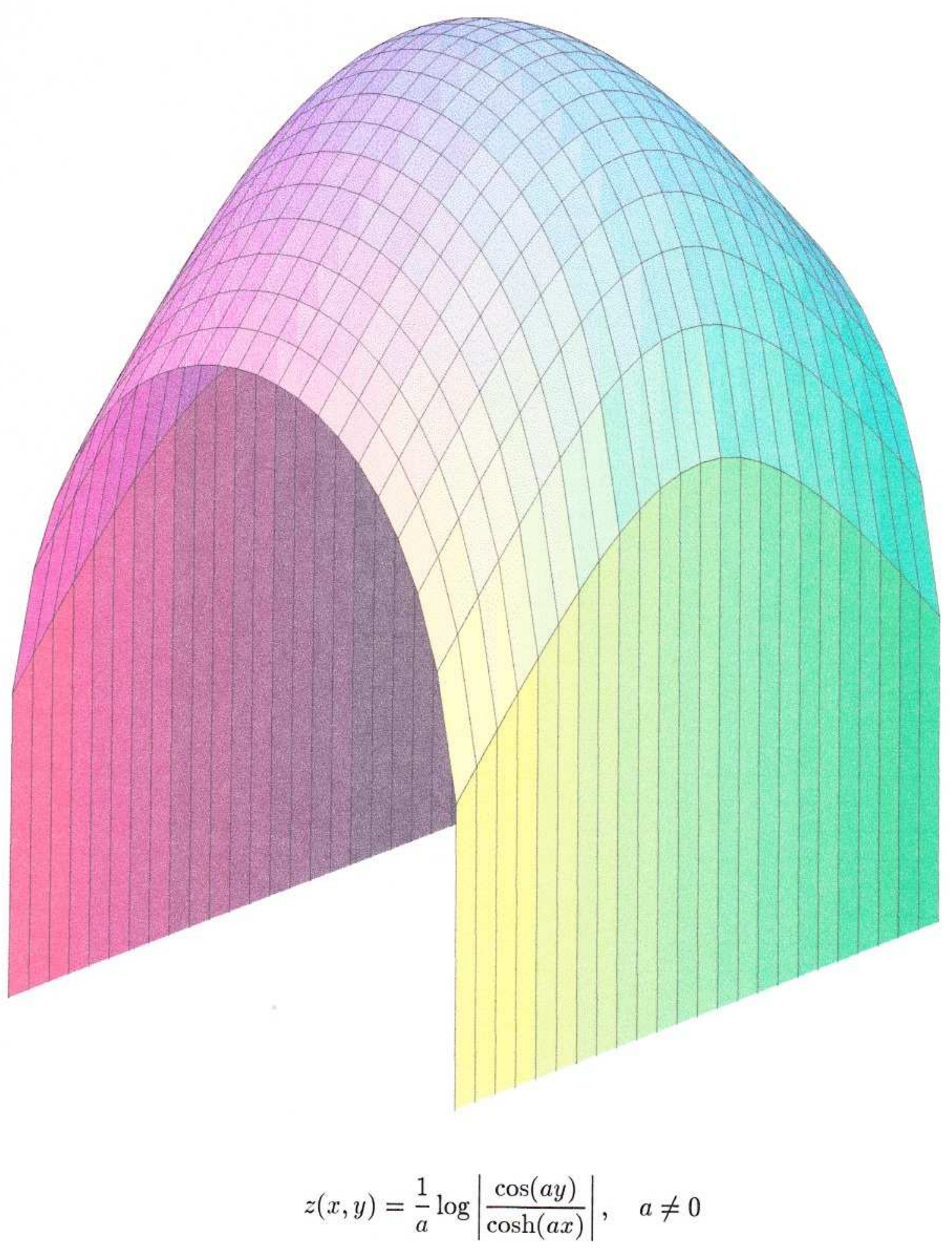


Figure 13 : A flat B-scroll over a null curve

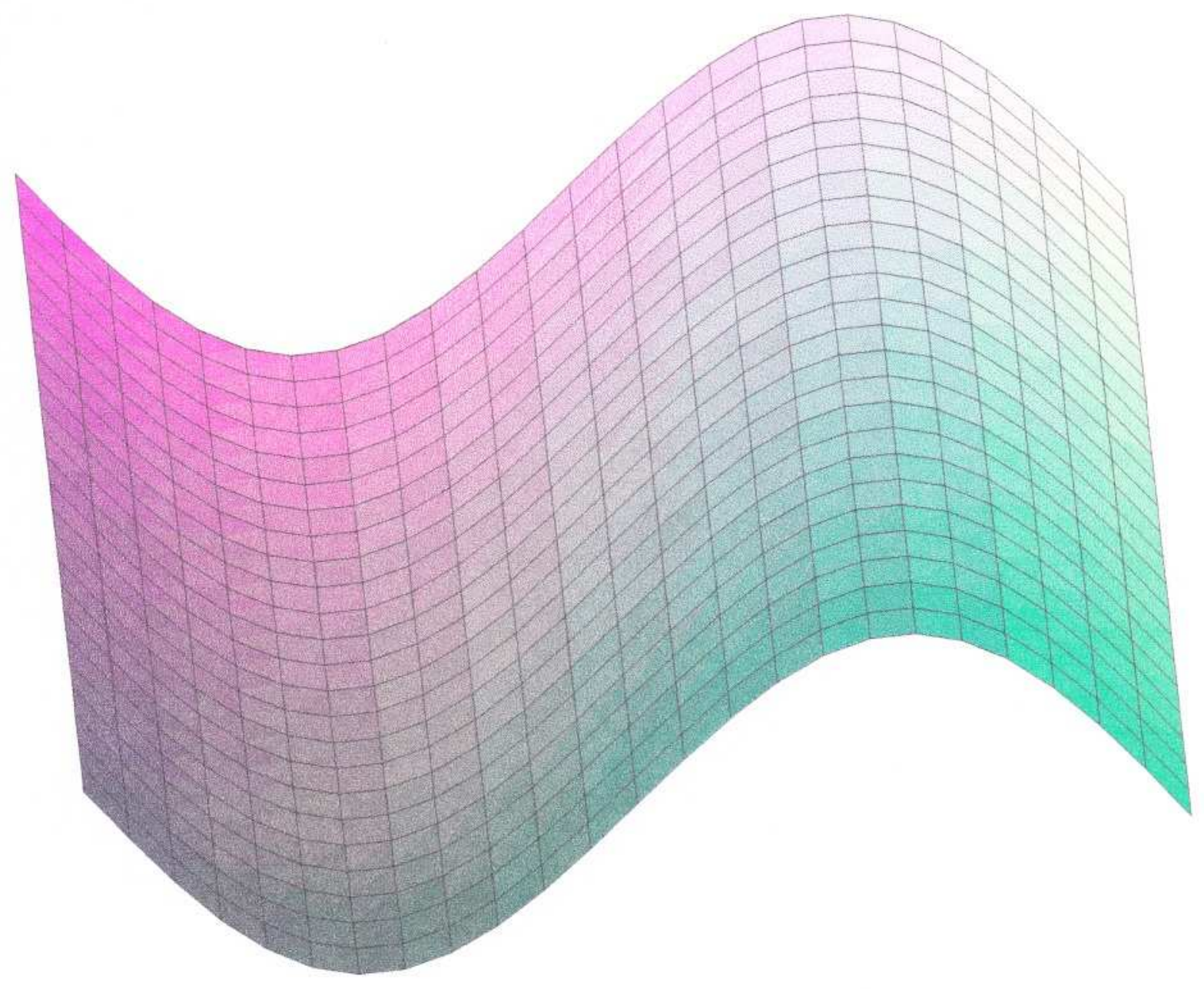

$$
z(x, y)= \pm x+g(y), \quad \text { with } g(y)=\sin y
$$


Figure 14: A circular cylinder

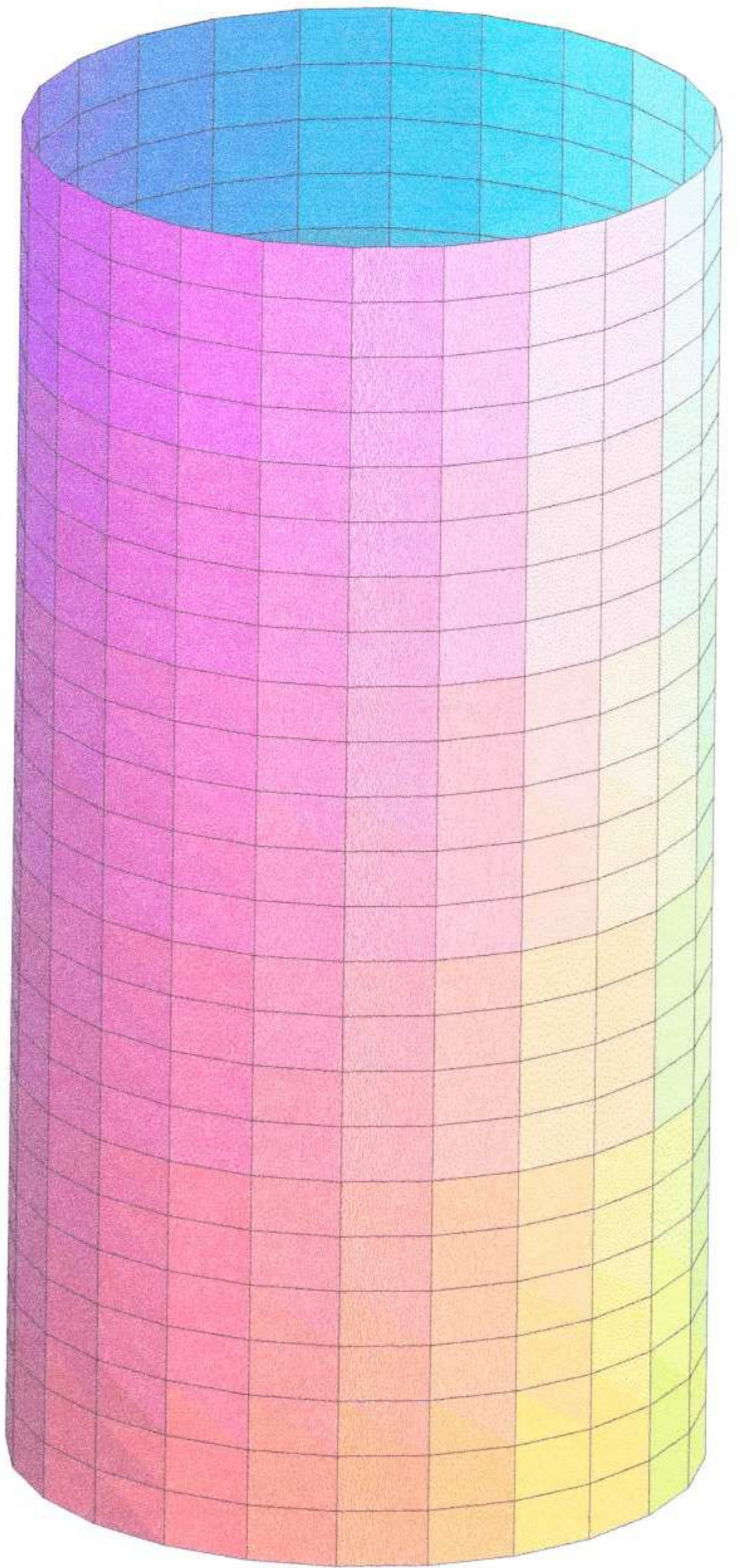

$$
X(s, t)=(a \cos s, a \sin s, b t), \quad a, b \in \mathbb{R}_{0}
$$


Figure 15 : A hyperbolic cylinder

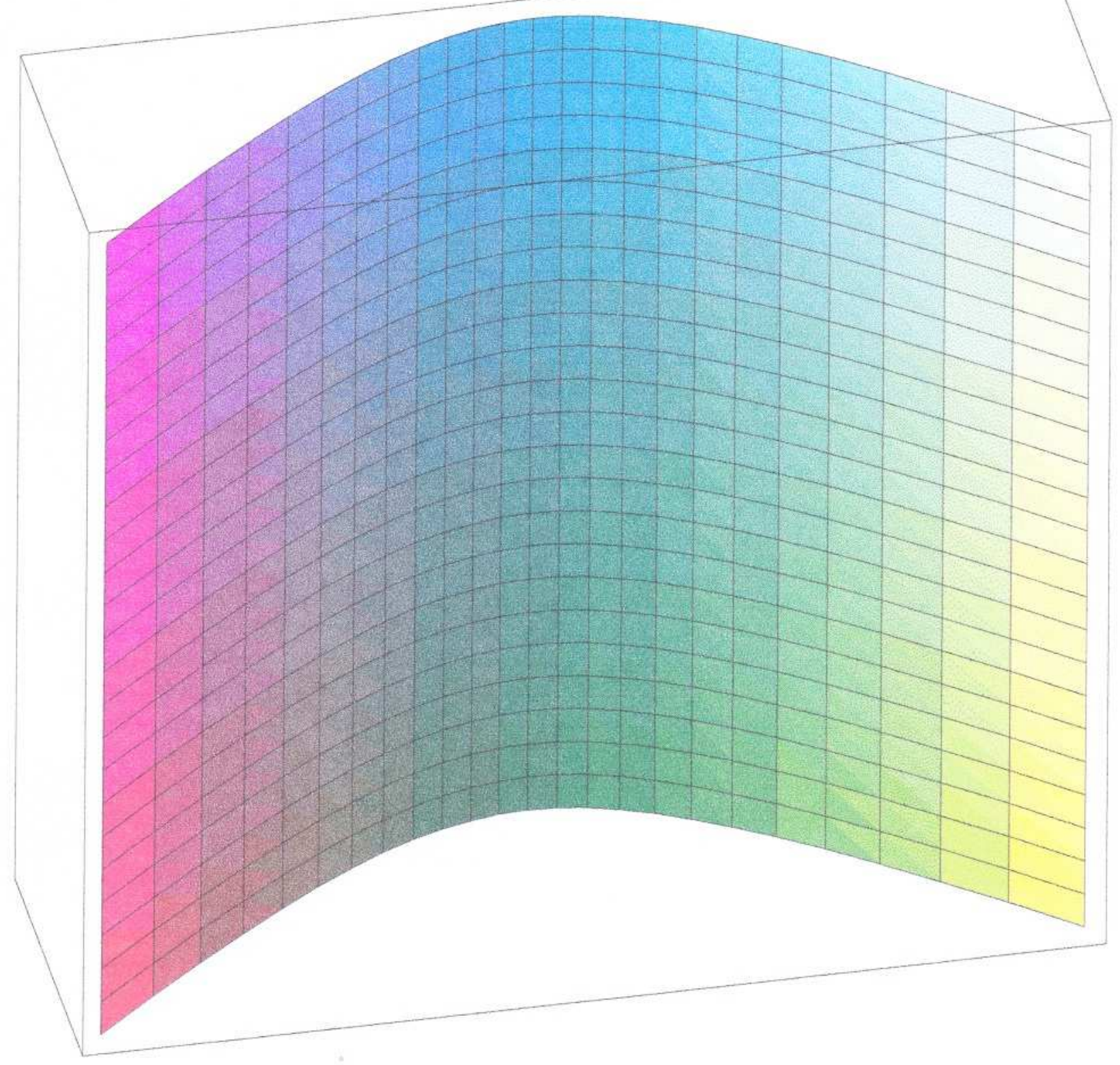

$X(s, t)=(a \cosh s, a \sinh s, b t), \quad a, b \in \mathbb{R}_{0}$ 
Figure 16 : An isoparametric surface with null rules : I

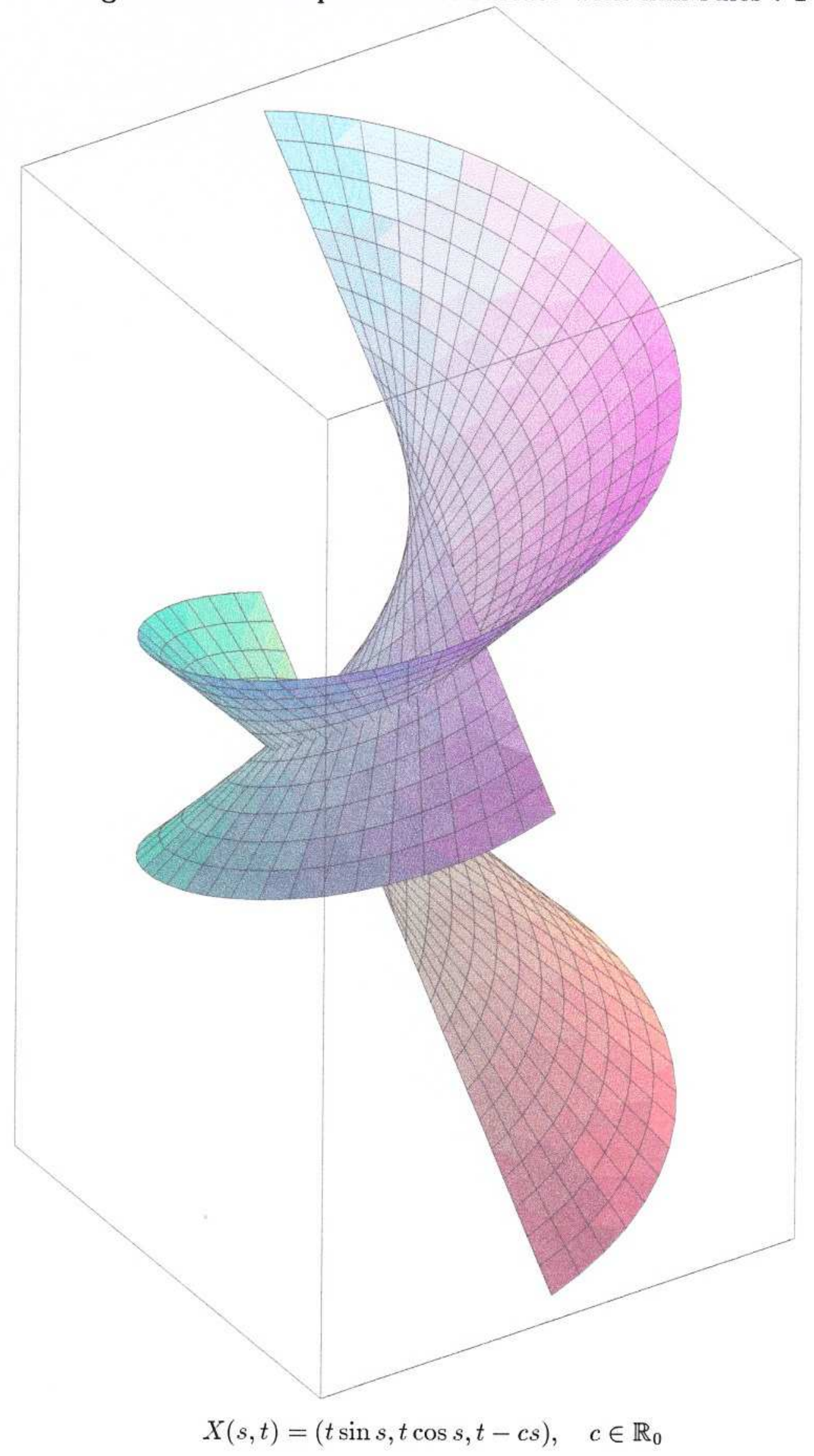


Figure 17 : An isoparametric surface with null rules : II

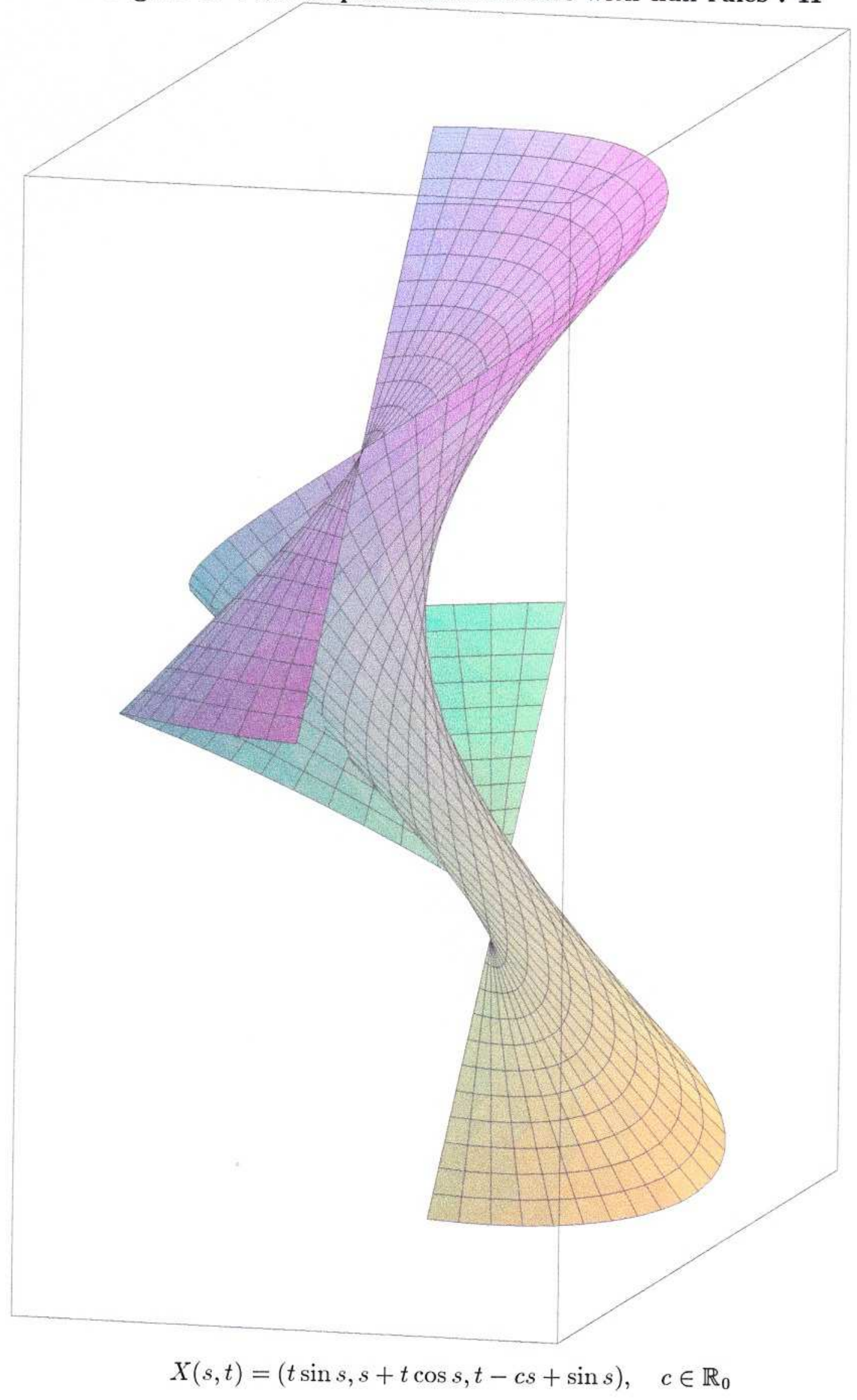


Figure 18 : An isoparametric surface with null rules : III

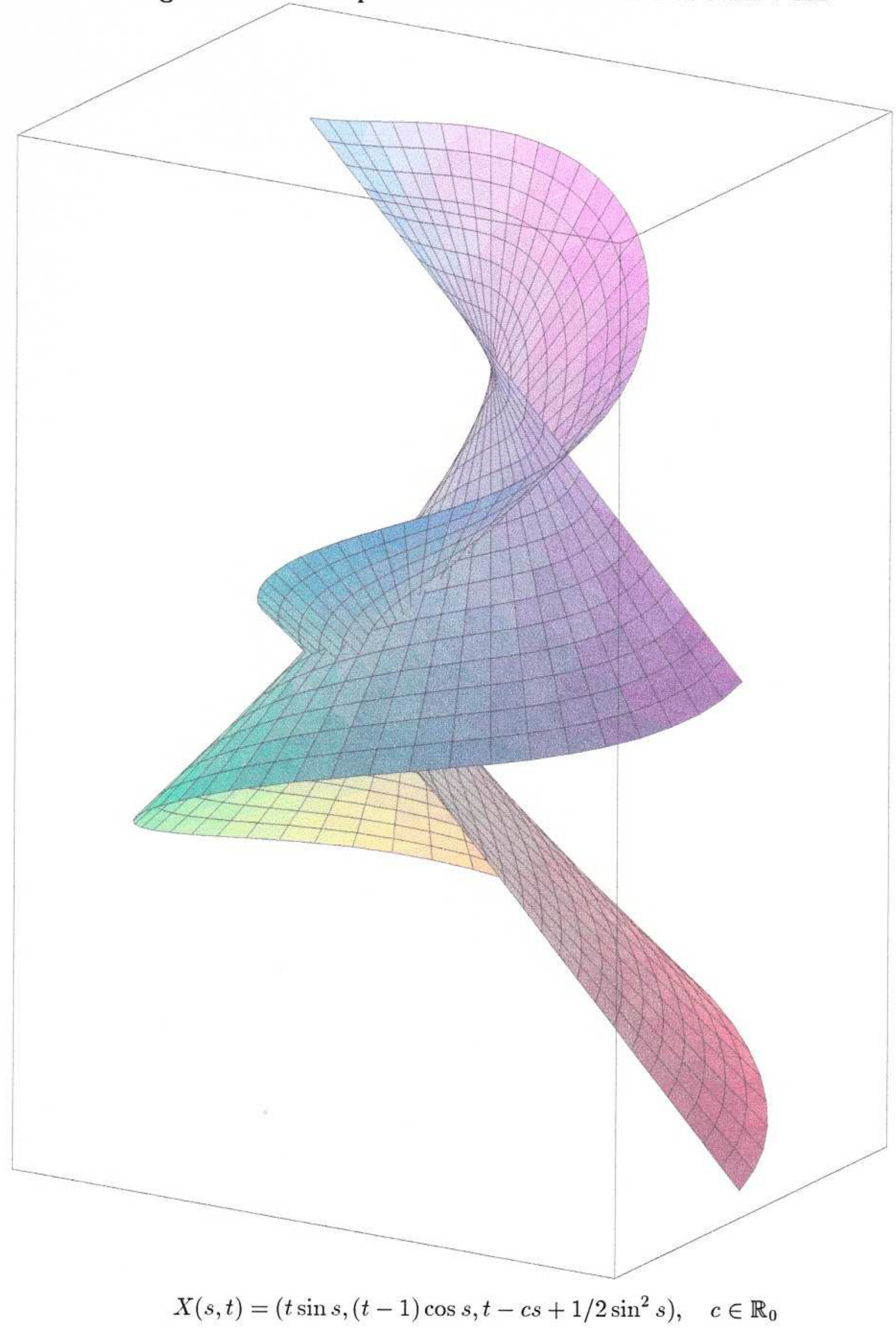




\section{REFERENCES}

[BC] M. Barros and B.Y. Chen, Spherical submanifolds which are of 2-type via the second standard immersion of the sphere, Nagoya Math. J., 108 (1987), 77-91.

[BD] J.L. Barbosa and M.P. Do Carno, Helicoids, catenoids and minimal hypersurfaces on $\mathbb{R}^{n}$ invariant by a 1-parameter group of motions, Ann. Acad. Brasil. Cienc. 53 (1981), 403-408.

[Bl] D. Blair, On a generalization of the catenoid, Can.J.Math. 27(1975), 231-236.

[Bo1] W.B. Bonner, Null curves in a Minkowski spacetime, Tensor, 20 (1969), 229-242.

[Bo2] W.B. Bonner, Curves with null normals in Minkowski spacetime, A random walk in relativity and cosmology, Wiley Easten Limited, 1985, 33-47.

[BU] M. Barros and F. Urbano,Special Geometry of minimal surfaces in the sphere, Tohoku Math. J., 39 (1987), 575-588.

[BV] D.E. Blair and J.R. Vanstone, A generalization of the helicoid in minimal submanifolds and geodesics, Kadai Publications, Tokyo, (1978), 13-16.

[Ch1] B.Y. Chen, Total mean curvature and submanifolds of finite type, World Scientific, Singapore, 1984.

[Ch2] B.Y. Chen, Geometry of slant submanifolds, K.U.Leuven,1990.

[Ch3] B.Y. Chen, Null 2-type surfaces in $\mathbb{E}^{3}$ are circular cylinders, Kodai Math. J., 11 2 (1988), 295-299.

[Ch4] B.Y. Chen, Geometry of Submanifolds, M. Dekker, New York, 1973.

[Ch5] B.Y. Chen, Differential Geometry of semiring of immersions, I: General Theory, Bull. Inst. Math. Acad. Sinica 21 (1993), 1-34.

[CDDVV] B.Y. Chen, J. Deprez, F. Dillen, L. Verstraelen and L. Vrancken, Curves of finite type, Geometry and Topology of submanifolds II, (1990), 76-110.

[CDV] B.Y. Chen, F. Dillen and L. Verstraelen, Finite type space curves, Soochow J. Math., 12 (1986), 1-10.

[CDVV1] B.Y. Chen, F. Dillen, L. Verstraelen and L. Vrancken, Ruled surfaces of finite type, Bull.Austral.Math.Soc., 42 (1990), 447-453.

[CDVV2] B.Y. Chen, F. Dillen, L. Verstraelen and L. Vrancken, Submanifolds of restricted type, Journal of Geometry, to appear.

[Di] F. Dillen,Ruled submanifolds of finite type, Proc.Amer.Math.Soc., 114 (1992), 795798.

[DDVV] F. Decruyenaere, F. Dillen, L. Verstraelen and L. Vrancken, The semiring of immersions of manifolds, Beiträge Algebra Geometrie 34 (1993), 209-215. 
[DPVV] F. Dillen, M. Petrovic, L. Verstraelen and L. Vrancken, Classification of curves of Chen type 2, Differential Geometry in honor of Radu Rosca, K.U.Leuven Departement Wiskunde, 1991, 101-107.

[DVVW1] F. Dillen, I. Van de Woestyne, L. Verstraelen, J. Walrave, Curves of restricted type in Minkowski space, Geometry and Topology of Submanifolds V, 1993, 161-168.

[DVVW2] F. Dillen, I. Van de Woestyne, L. Verstraelen, J. Walrave, Ruled surfaces of finite type in 3-dimensional Minkowski space, to appear.

[DVZ] F. Dillen, L. Verstraelen and G. Zafindratafa, A generalization of the translation surfaces of Scherk, Differential Geometry in the honor of Radu Rosca, K.U.Leuven,(1991), 107-109.

[IM] S. Ishikawa and S. Miyasato, On finite type curves in hyperbolic space, Soochow J.Math., 193 (1993), 339-356.

[Ko] O. Kobayashi, Maximal surfaces in the 3-dimensional Minkowski space $L^{3}$, Tokyo J. Math., 62 (1983), 297-309.

[Ma1] M. Magid, Lorentzian Isoparametric hypersurfaces, Pac.J.Math., 118 1(1985), 165-197.

[Ma2] M. Magid, Indefinite Einstein hypersurfaces with nilpotent shape operators, Hokkaido Math. Journal, 13 (1984), 241-250.

[Mi] I. Mihai, The study of tensor products of submanifolds reveals some close relations between differential geometry and ring theory, Bijgevoegde stelling, K.U. Leuven, 1993.

[MR] I. Mihai and B. Rouxel,Tensor product surfaces of Euclidean plane curves, to appear.

[MRVV] I. Mihai, R. Rosca, L. Verstraelen and L. Vrancken, Tensor product surfaces of Euclidean planar curves, to appear.

[MV] I. Mihai and L. Verstraelen, Introduction to tensor products of submanifolds, Geometry and Topology of Submanifolds VI, World Scientific Publ., 1994, 141-151.

[MVVW] I. Mihai, I. Van de Woestyne, L. Verstraelen and J. Walrave, Tensor product surfaces of Lorentzian planar curves, preprint.

[ON] B. O’Neill, Semi-Riemannian Geometry, Academic Press, New-York, 1983.

[Os] R. Osserman, A survey of minimal surfaces, Van Nostrand, New York, 1969.

[P] A.Z. Petrov, Einstein Spaces, Pergamon Press, Hungary, 1969.

[Ro] R. Rosca, Pseudo-umbilical two dimensional manifolds with m-index ${ }_{p} M=1$, immersed in a Minkowski space-time $M^{4}$, Tensor N.S. 24 (1972), 173-181.

[Ro1] B. Rouxel, Sur les courbes isotropes, pseudo-isotropes et les surfaces isotropes d'un espace-temps de Minkowski $M^{4}$, Rendiconti del Seminario della Facolta di Scienze dell' Universita di Cagliari, 49 (1979), 571-584.

[Ro2] B. Rouxel, Sur quelques proprié té anallagmatiques de l'espace Euclidien $\mathbb{E}^{4}$. 
[Ros] A. Ros, Eigenvalue inequalities for minimal submanifolds and P-manifolds, Math. Z., 191 (1984), 393-404.

[Sp] M. Spivak, $A$ comprehensive introduction to differential geometry, Publish or Perish, Houston, Texas, 1970.

[Ta] T. Takahashi, Minimal immersions of Riemannian manifolds, J. Math. Soc. Japan, 18 (1966), 380-385.

[Tai] S.S. Tai, Minimum imbeddings of compact symmetric spaces of rank one, J. Diff. Geometry, 2 (1968), 55-66.

[VdW1] I. Van de Woestyne, Krommingscondities op deelvarieteiten van semi-Euclidische ruimten en voorbeelden van minimale oppervlakken van de 3-dimensionale Minkowskiruimte, Doctoral thesis, 1989.

[VdW2] I. Van de Woestyne, Minimal surfaces of the 3-dimensional Minkowski space, Geometry and Topology of Submanifolds II, World Scientific Publ., 1990, 344-369.

[VVWY] I. Van de Woestyne, L. Verstraelen, J. Walrave and S. Yaprak, Minimal surfaces of translation in $\mathbb{R}_{1}^{n}$, Geometry and Topology of Submanifolds IV, World Scientific Publ., 1992, 217-228. 
KROMMEN EN OPPERVLAKKEN IN DE MINKOWSKIRUIMTE (BEKNOPTE SAMENVATTING) 


\section{§1. INLEIDING}

(In deze beknopte samenvatting zullen we proberen de belangrijkste resultaten van deze thesis terug te geven. Het is uiteraard niet mogelijk om alle resultaten met bewijs op te nemen in deze samenvatting. Daarom verwijzen we naar de Engelstalige versie indien $\mathrm{U}$ een bepaald onderwerp volledig uitgewerkt wil zien.)

Differentiaalmeetkunde is een jonge, snel evoluerende discipline binnen het meetkundeonderzoek. Alhoewel het begin van deze discipline kan gesitueerd worden in de $17 \mathrm{de}$ eeuw. Het was immers Isaac Newton die in 1671 de kromming van een kromme $\alpha$ voor het eerst uitdrukte in funktie van de afgeleiden van deze kromme $\alpha$. Newton beschouwde een kromme $\alpha(t)=\left(\alpha_{1}(t), \alpha_{2}(t)\right)$ als het traject beschreven door een punt dat beweegt in een vlak in funktie van de tijdparameter $t$. Hij definieerde de kromming als volgt. Neem twee punten $q_{1}$ en $q_{2}$ op de kromme, die dicht bij elkaar gelegen zijn, elk aan één kant van een gegeven punt $p$. Door deze drie punten gaat meestal juist één cirkel met middelpunt $m$. Wanneer de punten $q_{1}$ en $q_{2}$ naderen tot $p$, dan zal in de limiet een speciale cirkel bekomen worden, die raakt aan $\alpha$ in het punt $p$. Of met andere woorden, in het punt $p$ hebben $\alpha$ en deze cirkel dezelfde raaklijn. Deze cirkel is gekend als de osculatiecirkel of de kussende cirkel. Als $c$ het middelpunt is van deze osculatiecirkel en $r$ de straal van deze cirkel, dan definieerde Newton $\kappa=\frac{1}{r}$ als de kromming van deze kromme $\alpha$ in het punt $p$. Hij berekende $\kappa$ ook expliciet en bekwam de volgende uitdrukking

$$
\kappa=\frac{\dot{\alpha}_{1} \ddot{\alpha}_{2}-\ddot{\alpha}_{1} \dot{\alpha}_{2}}{\left(\dot{\alpha}_{1}^{2}+\dot{\alpha}_{2}^{2}\right)^{\frac{3}{2}}} .
$$

Men hoeft echter niet te werken met de parameter $t$, het was Euler die de booglengteparameter $s$ invoerde, zodanig dat $\dot{\alpha}_{1}^{2}+\dot{\alpha}_{2}^{2}=1$. Definieer dan $T(s)$ als het éénheidsvectorveld rakend aan $\alpha$, en $N(s)$, als het éénheidsvectorveld dat loodrecht staat op $T(s)$ in $\alpha(s)$ zodanig dat de oriëntatie van $\{T(s), N(s)\}$ dezelfde is als de standaardoriëntatie van $\mathbb{R}^{2}$. De kromming $\kappa(s)$ van $\alpha$ in $s$ is dan als volgt gedefinieerd

$$
\dot{T}(s)=\kappa(s) N(s) \text {. }
$$

Merk op dat $|\kappa(s)|=|\ddot{\alpha}(s)|$ de lengte is van $\ddot{\alpha}(s)$, die loodrecht staat op de éénheidsvector $\dot{\alpha}(s)$. Verder is $|\kappa(s)|$ ook de oppervlakte van de rechthoek gevormd door $\dot{\alpha}(s)$ en $\ddot{\alpha}(s)$. Deze oppervlakte is gelijk aan $\operatorname{det}(\dot{\alpha}(s), \ddot{\alpha}(s))$, dus hebben we dat

$$
\kappa(s)=\left|\begin{array}{cc}
\dot{\alpha}_{1}(s) & \ddot{\alpha}_{1}(s) \\
\dot{\alpha}_{2}(s) & \ddot{\alpha}_{2}(s)
\end{array}\right|=\dot{\alpha}_{1} \ddot{\alpha}_{2}-\ddot{\alpha}_{1} \dot{\alpha}_{2},
$$

wat dus overéénstemt met Newtons definitie. De congruentiestelling voor krommen gelegen in het Euclidisch vlak is als volgt, als er een continue funktie $\kappa$ met variabele $s$ gegeven wordt, dan bestaat er, op Euclidische transformaties na, een unieke kromme $\alpha$ in $\mathbb{E}^{2}$ met $s$ als booglengteparameter zodanig dat $\kappa(s)$ de kromming is van $\alpha$ in $\alpha(s)$. Dit betekent dat, als we rotaties en translaties buiten beschouwing laten, een kromme in $\mathbb{E}^{2}$ volledig bepaald wordt door haar kromming.

De studie van ruimtelijke krommen in $\mathbb{E}^{3}$, evolueerde enorm na de ontdekking van de Frenet-Serret formules. Deze formules werden door Frenet in 1847 en door Serret in 1851, 
onafhankelijk van elkaar, gevonden. Zij definieerden een orthonormaal referentiestelsel $\{T, N, B\}$ - gekend als het Frenetstelsel - in elk punt van de kromme $\alpha$, een ruimtelijke kromme geparameteriseerd door de booglengteparameter s. $T$ is het snelheidsvectorveld of nog het éénheidsvectorveld rakend aan $\alpha$. De versnelling $\ddot{\alpha}(s)$ van de kromme $\alpha$ in het punt $\alpha(s)$ is een vector die loodrecht staat op $T(s)$. Als $\ddot{\alpha}(s) \neq 0$, dan is het normale vectorveld $N$, het genormaliseerde versnellingsvectorveld $\ddot{\alpha}$. Het binormale vectorveld $B$ bekomt men door het vectorieel product van $T$ en $N$ te nemen. De Frenet-Serret formules worden dan gegeven door

$$
\begin{aligned}
\dot{T} & =\kappa N \\
\dot{N} & =-\kappa T+\tau B \\
\dot{B} & =-\tau N .
\end{aligned}
$$

De funkties $\kappa$ en $\tau$, die respectievelijk bepaald worden door de eerste en de derde vergelijking, worden de kromming en de torsie van de kromme genoemd. De congruentiestelling voor krommen in de 3-dimensionale Euclidische ruimte stelt : als $\kappa$ en $\tau$ twee continue funkties van $s$ zijn, dan bestaat er een kromme $\alpha$, booglengtegeparameteriseerd door $s$, met kromming $\kappa$ en torsie $\tau$. Deze stelling werd bewezen door Aoust in 1876 .

De studie van oppervlakken in $\mathbb{E}^{3}$ begon pas goed in 1760 dankzij Euler, die de kennis van krommen in het vlak gebruikte om oppervlakken te bestuderen. Door een punt $p$ op een oppervlak $M \subset \mathbb{E}^{3}$ construeren we een rechte $l$ loodrecht op het raakvlak aan $M$ in $p$. Voor elke éénheidsvector $X$ in het raakvlak, beschouwen we het vlak door $p$ dat zowel $X$ als $l$ bevat. De doorsnede van dit vlak met $M$ is het beeld van een kromme $\alpha_{X}$ met $\alpha_{X}(0)=p$. We veronderstellen dat $\alpha_{X}$ booglengtegeparameteriseerd is, zodanig dat $\dot{\alpha}_{X}(0)=X$. We oriënteren al deze vlakken door $X$ en $l$ door een vector $v_{p}$ te kiezen loodrecht op het raakvlak aan $M$ in $p$ en oriënteren het vlak door $X$ en $l$ zodanig dat $X, v_{p}$ positief georiënteerd is. $\alpha_{X}$ heeft dan een kromming in $s=0$, genoteerd $\kappa_{X}$. Euler ontdekte dat, als niet alle $\kappa_{X}$ gelijk zijn, er juist één richting bestaat, voorgesteld door de éénheidsvector $X_{1}$, waarin $\kappa_{X}$ een minimum bereikt $\kappa_{1}=\kappa_{X_{1}}$, en één richting waarin een maximum bereikt wordt $\kappa_{2}=\kappa_{X_{2}}$. Deze twee richtingen staan loodrecht op elkaar, en als $X$ een hoek $\theta$ vormt met $X_{1}$, dan is

$$
\kappa_{X}=\kappa_{1} \cos ^{2} \theta+\kappa_{2} \sin ^{2} \theta .
$$

50 jaar na Euler werd het belangrijkste werk in de geschiedenis van de differentiaalmeetkunde geschreven, de titel is " Disquisitiones generales circa superficies curvas" en Carl Friederich Gauss schreef het in 1827. In elk punt $p$ van $M$ is er een unieke vector $\nu(p)$ zodanig dat $\nu(p)$ loodrecht staat op het raakvlak aan $M$ in $p$ en zodanig dat $\nu(p), v, w$ positief georiënteerd is in $\mathbb{E}^{3}$ telkens als $v$ en $w$ positief georiënteerd zijn in het raakvlak aan $M$ in $p$. Gauss definieerde de normale afbeelding $\nu$ - gekend als de Gauss afbeelding - van het oppervlak $M$ naar $\mathbb{R}^{3}$, of beter naar de éénheidssfeer $S^{2} \subset \mathbb{R}^{3}$. De definitie van de kromming $K(p)$ van $M$ in $p$ wordt gegeven door

$$
K(p)=\lim _{A \rightarrow p} \frac{\operatorname{area} \nu(A)}{\operatorname{area} A}
$$


de limiet is zodanig dat het gebied $A$ rond $p$ smaller en smaller wordt. Gauss bewees dat deze kromming $K(p)$ in een willekeurig punt $p \in M$ het produkt is van de extreme krommingen van de krommen door $p$ bekomen als doorsnede van $M$ met normale vlakken,

$$
K(p)=\kappa_{1}(p) \kappa_{2}(p) .
$$

Het Theorema Egregium, of " Het merkwaardige Theorema ", van Gauss wordt als het belangrijkste resultaat uit de geschiedenis van de differentiaalmeetkunde beschouwd :

Theorema. De kromming $K(p)$ van een oppervlak is een intrinsieke invariant, dit wil zeggen is onafhankelijk van de ruimte waarin men het oppervlak bekijkt.

Gauss toonde aan dat de kromming $K(p)$ in een punt $p$ van een oppervlak in $\mathbb{E}^{3}$ een isometrische invariant is, dus niet enkel invariant onder Euclidische translaties en rotaties, maar ook invariant onder de veel grotere groep afbeeldingen, namelijk de isometriën van oppervlakken. De gemiddelde kromming $H(p)$ daarentegen,

$$
H(p)=\frac{1}{2}\left(\kappa_{1}(p)+\kappa_{2}(p)\right)
$$

is invariant onder de Euclidische transformaties, maar niet onder de isometriën van oppervlakken. Dus buiten de extrinsieke meetkunde van oppervlakken in $\mathbb{E}^{3}$, ontdekte Gauss een meetkundig begrip, zijn kromming, die de eerste invariant was voor de intrinsieke meetkunde van oppervlakken.

Georg Friederich Bernhard Riemann zette Gauss's meesterwerk in de praktijk om en ontwikkelde differentiaalmeetkunde voor objecten die niet noodzakelijk in de Euclidische ruimte moeten gelegen zijn. Hij slaagt erin van een lengtefunktie te verbinden aan elke rakende ruimte, dus een metriek, een norm, ... . Het eenvoudigste geval, wanneer de metriek positief-definiet is, wordt door Riemann zelf uitvoerig bestudeerd. Het definiëren van een inwendig produkt voor elke rakende ruimte aan een variëteit is wat we nu kennen als een Riemannse metriek op een variëteit $M$. De daaraan gekoppelde intrinsieke meetkunde is gekend als de Riemannse meetkunde.

Indien de metriek op een differentieerbare variëteit indefiniet is, dan spreken we van een semi-Riemannse variëteit, de corresponderende meetkunde is de semi-Riemannse meetkunde. Een semi-Riemannse variëteit kan beschouwd worden als een deelvariëteit in een semi-Euclidische ruimte, we kunnen hier dus ook intrinsiek als extrinsiek werken.

De Minkowskiruimte $\mathbb{E}_{1}^{n}$, i.e. de variëteit $\mathbb{R}^{n}$ voorzien van een metrische tensor $g$ van index 1 , is een zeer belangrijke semi-Riemannse variëteit. Als $n=4$, is het het voorbeeld van een relativistische tijdruimte.

In het eerste hoofdstuk van deze thesis bestuderen we krommen in de drie en vier dimensionale Minkowskiruimte.

Het bewegend referentiestelsel van Frenet voor krommen in de Minkowskiruimte wordt bestudeerd. Het causaal karakter van de kromme zal hier een belangrijke rol in spelen.

In paragraaf drie classificiëren we alle krommen met constante Frenet-krommingen in de drie dimensionale Minkowskiruimte. De nulkrommen hebben maar één kromming, wat toch uitzonderlijk is. Als deze kromming nul is, bekomen we de gekende nulkubiek. 
In de vierde en vijfde paragraaf behandelen we krommen van beperkt type en krommen van eindig type. Resultaten van krommen van eindig type in de Euclidische ruimte werden onder andere gevonden door B.Y. Chen, die het begrip ook invoerde. Zo bewees hij dat een gesloten kromme in de Euclidische ruimte van eindig type is als en slechts als de Fourierreeks van elke coördinaatfunktie van de kromme een eindig aantal van nul verschillende termen heeft. Als deze kromme $\alpha$ een gesloten vlakke kromme is, dan is ze van eindig type als en slechts als ze van type 1 is en dus een cirkel is. Chen, Deprez, Dillen, Verstraelen en Vrancken [CDDVV] bestudeerden ruimtelijke krommen van eindig type. $\mathrm{Zij}$ toonden aan dat voor elk positief geheel getal $\mathrm{k}$, er in $\mathbb{E}^{3}$ een gesloten kromme bestaat van type $\mathrm{k}$. Wij bewijzen dat alle krommen van eindig type in het Minkowskivlak van type 1 zijn, en dus een deel van een orthogonale hyperbool of een deel van een rechte zijn. Bovendien geven we een classificatie van alle krommen van type 2 in $\mathbb{E}_{1}^{3}$.

Oppervlakken in de drie en $n$-dimensionale Minkowskiruimte bestuderen we in hoofdstuk twee.

Eerst beschouwen we minimale oppervlakken, oppervlakken met een gemiddelde kromming $H=0$. Kobayashi [Ko] en Van de Woestyne [VdW1] gaven een classificatie van alle minimale omwentelingsoppervlakken in $\mathbb{E}_{1}^{3}$, alle minimale regeloppervlakken in $\mathbb{E}_{1}^{3}$ en alle minimale translatieoppervlakken in $\mathbb{E}_{1}^{3}$ met metriek $g=d x^{2}+d y^{2}-d z^{2}$. Een translatieoppervlak $M$ in $\mathbb{E}_{1}^{n}$ is een oppervlak gegeven door de volgende immersie

$$
X: U \subset \mathbb{E}^{2} \rightarrow \mathbb{E}_{1}^{n}:(x, y) \rightarrow\left(x, y, f_{3}(x)+g_{3}(y), \ldots, f_{n}(x)+g_{n}(y)\right) .
$$

Wij geven een classificatie van alle minimale translatieoppervlakken in de $n$-dimensionale Minkowskiruimte $\mathbb{E}_{1}^{n}$ met metriek $g=-d x_{1}^{2}+\sum_{i=2}^{n} d x_{i}^{2}$.

In [CDVV1] is bewezen dat een regeloppervlak van eindig type in $\mathbb{E}^{n}$ ofwel een deel van een cilinder over een kromme van eindig type is, ofwel ee helicoïde in $\mathbb{E}^{3}$ is. Voor $n=3$ hebben we dus dat een regeloppervlak in $\mathbb{E}^{3}$ van eindig type is als en slechts als het een deel is van een vlak, of een cirkulaire cilinder of een helicoide. Dillen [Di] toonde aan dat een regeldeelvariëteit van eindig type in een Euclidische ruimte is als en slechts als het een cilinder is op een kromme van eindig type of een veralgemeende helicoïde. In paragrafe 3 van hoofdstuk twee geven we een classificatie van alle regeloppervlakken van eindig type in $\mathbb{E}_{1}^{3}$. Buiten de minimale oppervlakken en de te verwachten cirkulaire en hyperbolische cilinders, bekomen we een andere klasse van regeloppervlakken van eindig type, namelijk de isoparametrische regeloppervlakken met nulregels. De gemiddelde kromming $H$ en de Gausskromming $K$ zijn constant voor deze oppervlakken en ze zijn van type 1 of van het nul 2 type. We tonen verder aan dat een isoparametrisch oppervlak met nulregels van type 1 is als en slechts als het een éénbladige hyperboloïde is.

In de laatste paragrafe van hoofdstuk twee bestuderen we regeloppervlakken met constante gemiddelde kromming in $\mathbb{E}_{1}^{3}$. De minimale regeloppervlakken behoren tot deze klasse en ook de regeloppervlakken van eindig type behoren tot deze klasse. We bewijzen dat een regeloppervlak in $\mathbb{E}_{1}^{3}$ constante gemiddelde kromming heeft als en slechts als het van eindig type is.

In het laatste hoofdstuk onderzoeken we tensorproduktoppervlakken in $\mathbb{E}_{2}^{4}$, afkomstig van een Lorentzkromme en een Euclidische kromme. Stel dat $\alpha: \mathbb{R} \rightarrow \mathbb{E}_{1}^{2}$ en $\beta: \mathbb{R} \rightarrow \mathbb{E}^{2}$ 
respectievelijk een vlakke Lorentz kromme en een vlakke Euclidische kromme is. Als $\alpha(t)=\left(\alpha_{1}(t), \alpha_{2}(t)\right)$ en $\beta(s)=\left(\beta_{1}(s), \beta_{2}(s)\right)$, dan wordt hun tensorprodukt gegeven door

$$
\alpha \otimes \beta: \mathbb{R}^{2} \rightarrow \mathbb{E}_{2}^{4}:(t, s) \rightarrow\left(\alpha_{1}(t) \beta_{1}(s), \alpha_{1}(t) \beta_{2}(s), \alpha_{2}(t) \beta_{1}(s), \alpha_{2}(t) \beta_{2}(s)\right.
$$

De minimale tensorproduktoppervlakken bespreken we in paragrafe 2. Een volledige classificatie van deze klasse van oppervlakken wordt gegeven. Het tensorprodukt $\alpha \otimes \beta$ is een minimale immersie als en slechts als ofwel $\alpha$ of $\beta$ een rechte door de oorsprong is, ofwel zowel $\alpha$ als $\beta$ een orthogonale hyperbool met middelpunt de oorsprong is, ofwel $\beta$ een cirkel met middelpunt de oorsprong is en $\alpha$ ofwel een cirkel ofwel een hyperbool met middelpunt de oorsprong is.

In de volgende paragrafe bestuderen we totally real en complex tensorproduktoppervlakken. Deze twee klassen zijn speciale gevallen van slant oppervlakken. We bewijzen dat het tensorprodukt $\alpha \otimes \beta$ een totally real immersie t.ov. de pseudo-Hermitische structuur $J_{0}$,

$$
J_{0}(u, v, z, w)=(-v, u,-w, z),
$$

op $\mathbb{E}_{2}^{4}$ is als en slechts als $\alpha$ een orthogonale hyperbool met middelpunt de oorsprong is, ofwel als $\beta$ een rechte door de oorsprong is. Verder bewijzen we dat het tensorprodukt $\alpha \otimes \beta$ een complexe immersie t.o.v. $J_{0}$ op $\mathbb{E}_{2}^{4}$ is als en slechts als $\alpha$ een rechte door de oorsprong is.

Verder bestuderen we nog pseudo-minimale en pseudo-umbilicale tensorproduktoppervlakken. We bekomen dat een tensorprodukt $\alpha \otimes \beta$ een niet-minimale pseudominimale immersie is als en slechts als $\alpha$ een hyperbolische spiraal in een Minkowskivlak is en $\beta$ een logarithmische spiraal in een Euclidisch vlak is. Verder bewijzen we dat het tensorprodukt van een hyperbool en een logarithmische spiraal een pseudo-umbilicale immersie is in $\mathbb{E}_{2}^{4}$. En analoog is het produkt van een cirkel en een hyperbolische spiraal een pseudo-umbilicale tensorproduktimmersie in $\mathbb{E}_{2}^{4}$. 


\section{§2. KROMMEN MET CONSTANTE KROMMINGEN}

Een één-dimensionale deelvariëteit of met andere woorden een kromme gelegen in een willekeurige variëteit $M$ wordt als volgt gedefinieerd.

Definitie 1. Een kromme gelegen in een variëteit $M$ is een differentieerbare functie $\alpha: I \rightarrow M$, met $I$ een open interval in $\mathbb{R}$.

Wanneer een kromme gelegen is in een semi-Riemannse variëteit dan kan zij verschillende causale karakters aannemen, zoals blijkt uit de volgende definitie.

Definitie 2. Een kromme $\alpha$ gelegen in een semi-Riemannse variëteit $M$ met metriek $g$ is

(1) ruimtelijk als en slechts als $g(\dot{\alpha}(s), \dot{\alpha}(s))>0$ of $\dot{\alpha}(s)=0$ voor elke $s$,

(2) $n u l$ als en slechts als $g(\dot{\alpha}(s), \dot{\alpha}(s))=0$ voor elke $s$ met $\dot{\alpha}(s) \neq 0$,

(3) Lorentz als en slechts als $g(\dot{\alpha}(s), \dot{\alpha}(s))<0$ voor elke $s$.

Het is niet zo dat een kromme altijd juist één van deze causale karakters heeft, zij kan evengoed in een bepaald gebied ruimtelijk zijn en in een ander gebied Lorentz.

We kunnen een kromme beschrijven ten opzichte van een vast referentiestelsel, maar ook ten opzichte van een bewegend referentiestelsel. We beschouwen eerst het bewegend Frenet referentiestelsel in $\mathbb{E}^{3}$.

Beschouw een kromme $\alpha(s)=\left(\alpha_{1}(s), \alpha_{2}(s), \alpha_{3}(s)\right)$, met $s$ de booglengteparameter voor $\alpha$. Dit betekent dat $\forall s: g(\dot{\alpha}, \dot{\alpha})=1$. Deze kromme ligt in de 3-dimensionale Euclidische ruimte $\mathbb{E}^{3}$, voorzien van de standaard metriek $g=d x_{1}^{2}+d x_{2}^{2}+d x_{3}^{2}$. Het Frenet referentiestelsel voor $\alpha$ is het orthonormale referentiestelsel $\{T, N, B\}$, dat als volgt geconstrueerd wordt. $T$ is het éénheidsvectorveld rakend aan $\alpha$, ook wel eens snelheidsvectorveld genoemd. De versnellingsvector $\ddot{\alpha}(s)$ van de kromme $\alpha$ in het punt $\alpha(s)$ is een vector die loodrecht staat op de vector $T(s)$ in dat punt $\alpha(s)$. Het hoofdnormaalvectorveld $N(s)$ is niets anders dan het genormaliseerde versnellingsvectorveld $\ddot{\alpha}$. Het binormale vectorveld $B(s)$ is tenslotte gedefinieerd als het vectorieel product van $T(s)$ met $N(s)$. De formules van Frenet geassocieerd aan dit referentiestelsel zijn dan

$$
\begin{aligned}
\dot{T} & =\kappa N \\
\dot{N} & =-\kappa T+\tau B \\
\dot{B} & =-\tau N
\end{aligned}
$$

of nog

$$
\left(\begin{array}{l}
\dot{T} \\
\dot{N} \\
\dot{B}
\end{array}\right)=\left(\begin{array}{ccc}
0 & \kappa & 0 \\
-\kappa & 0 & \tau \\
0 & -\tau & 0
\end{array}\right) \cdot\left(\begin{array}{c}
T \\
N \\
B
\end{array}\right)
$$

en bepalen alle verdere afgeleiden van $\alpha$. De functies $\kappa(s)$ en $\tau(s)$, die bepaald worden door de eerste en de derde formule, worden respectievelijk de eerste en de tweede 
kromming van $\alpha$ genoemd, of nog beter de kromming en de torsie. De hoofdstelling voor krommen in $\mathbb{E}^{3}$ stelt dat twee krommen met dezelfde kromming en torsie congruent zijn.

Wat Frenets systeem voor krommen in een Euclidische ruimte doet, is eigenlijk niets anders dan in elk punt van de kromme een aangepast referentiestelsel definiëren en dan de afgeleide van de bekomen referentievectorvelden uitdrukken ten opzichte van deze referentievectorvelden. Met als gevolg dat de krommingen van deze kromme, die deze kromme volledig bepalen, opduiken als coëfficiënten in deze formules.

Ook voor krommen gelegen in de drie-dimensionale Minkowskiruimte $\mathbb{E}_{1}^{3}$ kunnen we een Frenet referentiestelsel definiëren en de bijhorende Frenet formules afleiden. Het causaal karakter van de kromme speelt hierin een belangrijke rol. Bij wijze van voorbeeld behandelen we hier het geval waarin de kromme een nul kromme is.

Gegeven: een nul kromme $\alpha$

Het vectorveld $T(s)$ stellen we gelijk aan het nul vectorveld $\dot{\alpha}(s)$. Dan weten we dat $\ddot{\alpha}(s)$ een ruimtelijk vectorveld is dat loodrecht staat op het vectorveld $T(s)$, behalve als $\ddot{\alpha}(s)=0$ voor elke $s$ (wat dus betekent dat $\alpha(s)$ een nul rechte is). Indien $\alpha(s)$ geen nul rechte is, kunnen we de parameter $s$ zodanig kiezen dat $g(\ddot{\alpha}(s), \ddot{\alpha}(s))=1, \quad \forall s ; s$ wordt dan de pseudo-booglengteparameter genoemd voor $\alpha$. In dit geval definiëren we $N(s)$ als het éénheidsvectorveld $\ddot{\alpha}(s)$. Het binormaal vectorveld $B(s)$ is het unieke nul vectorveld dat loodrecht staat op het vectorveld $N(s)$ in elk punt $\alpha(s)$ van $\alpha$, zodanig dat $g(T(s), B(s))=1$.

De Frenet formules kunnen dan als volgt afgeleid worden,

$$
\left(\begin{array}{c}
\dot{T} \\
\dot{N} \\
\dot{B}
\end{array}\right)=\left(\begin{array}{ccc}
0 & \kappa_{1} & 0 \\
\kappa_{2} & 0 & -\kappa_{1} \\
0 & -\kappa_{2} & 0
\end{array}\right) \cdot\left(\begin{array}{l}
T \\
N \\
B
\end{array}\right),
$$

let wel, de "kromming" $\kappa_{1}$ kan maar twee waarden aannemen; 0 , als $\alpha$ een nul rechte is, of 1 in alle andere gevallen. Wat dus betekent dat een nul kromme in $\mathbb{E}_{1}^{3}$ eigenlijk maar één echte kromming heeft.

Men kan dan gaan kijken naar de krommen waarvoor deze krommingen constant zijn. Wij hebben dit gedaan voor alle krommen in $\mathbb{E}_{1}^{3}$. Hier bekijken we opnieuw enkel het speciale geval wanneer $\alpha(s)$ een nul kromme is. De classificatie van nul krommen in $\mathbb{E}_{1}^{3}$ met constante krommingen wordt gegeven in de volgende stelling.

Stelling 3. Een nul kromme in $\mathbb{E}_{1}^{3}$, met metriek $g=-d x^{2}+d y^{2}+d z^{2}$, met constante krommingen ziet er als volgt uit

(1) $\kappa_{1}=0$ als en slechts als $\alpha(s)$ is een deel van een nul rechte;

(2) $\kappa_{1}=1$ en $\kappa_{2}=0$ als en slechts als $\alpha(s)$ is een deel van een nul kubiek met de volgende parameterisatie,

$$
\alpha(s)=\frac{1}{6 \sqrt{2}}\left(6 s+s^{3}, 3 \sqrt{2} s^{2}, 6 s-s^{3}\right) ;
$$

(3) $\kappa_{1}=1$ en $\kappa_{2}>0$ als en slechts als $\alpha(s)$ een deel is van een circulaire nul helix, geparameteriseerd door

$$
\alpha(s)=\frac{1}{K^{2}}(K s, \cos (K s), \sin (K s))
$$


met $K=\sqrt{2 \kappa_{2}}$;

(4) $\kappa_{1}=1$ en $\kappa_{2}<0$ als en slechts als $\alpha(s)$ een deel is van een hyperbolische nul helix, met parameterisatie

$$
\alpha(s)=\frac{1}{K^{2}}(\sinh (K s), K s, \cosh (K s))
$$

met $K=\sqrt{-2 \kappa_{2}}$.

Bewijs. Als $\kappa_{2}=0$, dan ziet de Maclaurin reeksontwikkeling van $\alpha(s)$ er als volgt uit

$$
\alpha(s)=\alpha(0)+T(0) s+N(0) \frac{s^{2}}{2}-B(0) \frac{s^{3}}{6}
$$

met $\{T(s), N(s), B(s)\}$ het Frenet referentiestelsel voor $\alpha$. Dus $T(s)$ en $B(s)$ zijn twee nul vectorvelden, zodanig dat $g(T(s), B(s))=1 . N(s)$ is een genormaliseerd ruimtelijk vectorveld loodrecht op $T(s)$ en $N(s)$. We kunnen er dus voor zorgen dat voor $s=0$,

$$
T(0)=\frac{1}{\sqrt{2}}(1,0,1), \quad N(0)=(0,1,0), \quad B(0)=\frac{1}{\sqrt{2}}(-1,0,1) .
$$

Wat dus moest bewezen worden.

Als $\kappa_{2} \neq 0$, dan definiëren we

$$
\alpha_{0}(s)=\alpha(s)-\frac{1}{2 \kappa_{2}} N(s)
$$

Dit is een rechte. Het causaal karakter van deze rechte is afhankelijk van het teken van de kromming $\kappa_{2}$. Als $\kappa_{2}<0$, dan is $\alpha_{0}(s)$ een Lorentz rechte en kan dus, zonder afbreuk te doen aan de algemeenheid, beschouwd worden als de $\mathrm{X}$-as. In het andere geval is $\alpha_{0}(s)$ een ruimtelijke rechte, bijvoorbeeld de $\mathrm{Y}$-as. In beide gevallen staat het ruimtelijke éénheidsvectorveld $N(s)$ loodrecht op $\dot{\alpha}_{0}$. Wat betekent dat $N(s)$ in beide gevallen een specifieke vorm aanneemt. Uit de definitie van $\alpha_{0}$ volgt dat als we $\alpha_{0}(s)$ en $N(s)$ kennen, we ook $\alpha(s)$ kennen. 


\section{§3. KROMMEN VAN RESTRICTED EN EINDIG TYPE}

Een deelvariëteit $N^{n}$ in een Euclidische ruimte $\mathbb{E}^{m}$ is van restricted type (R.T.) als voor elke raakvector $X$ in om het even welk punt $\mathrm{p}$ van $N^{n}$ geldt dat

$$
A_{H}(X)=(M X)^{T},
$$

met $A_{H}$ de shape-operator of Weingartenfunctie van $N^{n}$ in het punt p ten opzichte van het gemiddeld krommingsvectorveld $H$. De notatie ()$^{T}$ staat voor de rakende component en $M \in \mathbb{R}^{m \times m}$ is een vast endomorfisme in de omgevende ruimte $\mathbb{E}^{m}$ [CDVV]. Voor een kromme $\alpha(s)$ in $\mathbb{E}^{2}$, met standaard metriek $g$, geparameteriseerd door de booglengteparameter $s$, wordt de bovenstaande definitie

$$
\kappa^{2}=g(M T, T),
$$

met $M$ een vast endomorfisme in $\mathbb{E}^{2}, \quad T(s)=\dot{\alpha}(s)$ en $\kappa(s)$ is de kromming van $\alpha(s)$. Een complete classificatie van restricted type krommen in het Euclidische vlak $\mathbb{E}^{2}$ vindt men in [CDVV2].

Hetzelfde kan gedaan worden in het Minkowskivlak, vandaar dat wij een classificatie gevonden hebben van alle vlakke krommen van restricted type in de 2-dimensionale Minkowskiruimte $\mathbb{E}_{1}^{2}$, dit is $\mathbb{R}^{2}$ voorzien van de Lorentz metriek $g=d x^{2}-d y^{2}$.

Beschouw een kromme $\alpha(s)$ in $\mathbb{E}_{1}^{2}$. Omdat we in een Minkowskiruimte werken moeten we rekening houden met het causale karakter van $\alpha(s)$. Wij zullen echter maar twee gevallen in beschouwing nemen, namelijk de ruimtelijke en de Lorentz krommen, de enige nul krommen in $\mathbb{E}_{1}^{2}$ zijn immers de nul rechten.

We geven eerst de aangepaste definitie.

Een kromme $\alpha(s)$, geparameteriseerd door de booglengteparameter $s$, is van restricted type in $\mathbb{E}_{1}^{2}$ als er een vast endomorfisme $M \in \mathbb{R}^{2 \times 2}$ bestaat zodat

$$
-\kappa^{2}=g(M T, T),
$$

met $\kappa$ de kromming uit de Frenet formules voor $\alpha(s)$ en $T(s)=\dot{\alpha}(s)$. Wegens de symmetrie van de shape-operator $A_{H}$ in de oorspronkelijke definitie, mogen we veronderstellen dat $M$ een zelf-toegevoegde operator is, eventueel door $M$ te vervangen door

$$
\tilde{M}=\frac{1}{2}\left(M+\varepsilon M^{t} \varepsilon\right)
$$

met

$$
\varepsilon=\left(\begin{array}{cc}
-1 & 0 \\
0 & 1
\end{array}\right)
$$

Dankzij een gekend theorema van Petrov [Pe], weten we dat $M$ één van de volgende vormen aanneemt ( het is namelijk niet zo dat in een Minkowskiruimte elke symmetrische, lineaire operator diagonaliseerbaar is) :

$$
M=\left(\begin{array}{ll}
a & 0 \\
0 & b
\end{array}\right),
$$


ten opzichte van een orthonormale basis;

$$
M=\left(\begin{array}{cc}
a & b \\
-b & a
\end{array}\right),
$$

ten opzichte van een orthonormale basis en met $b \neq 0$;

$$
M=\left(\begin{array}{ll}
a & 0 \\
1 & a
\end{array}\right),
$$

ten opzichte van een pseudo-orthonormale basis $u_{1}, u_{2}$;

$g\left(u_{1}, u_{1}\right)=g\left(u_{2}, u_{2}\right)=0 ; \quad g\left(u_{1}, u_{2}\right)=-1 ;$ met $a, b \in \mathbb{R}$.

Met behulp van deze classificatiestelling van Petrov, kunnen wij nu alle krommen van restricted type in $\mathbb{E}_{1}^{2}$ bepalen. Daarvoor gebruiken we echter eerst de volgende stelling.

Stelling 4. Als $\alpha(s)$ een kromme van restricted type is in $\mathbb{E}_{1}^{2}$, dan is de spiegeling van $\alpha(s)$ ten opzichte van één van de bissectrices ook een kromme van restricted type in $\mathbb{E}_{1}^{2}$.

Dit heeft als gevolg dat als we alle ruimtelijke krommen van restricted type kennen, we meteen ook alle Lorentz krommen van restricted type kennen, en vice versa. Vandaar dat we enkel een classificatiestelling geven van alle ruimtelijke krommen van restricted type.

Stelling 5. Een ruimtelijke kromme in $\mathbb{E}_{1}^{2}$ is van restricted type als en slechts als het een deel is van één van de volgende krommen :

(1) een rechte;

(2) een orthogonale hyperbool;

krommen met vergelijking :

(3) $\sinh (c y)=e^{c x}, \quad c \neq 0$;

(4) $\cosh (c x)=e^{c y}, \quad c \neq 0$;

(5) $-a \cosh ^{2}(\sqrt{c} x)+b \cosh ^{2}(\sqrt{c} y)=c, \quad b>a>0, \quad c=b-a$;

(6) $a \sinh ^{2}(\sqrt{c} x)+b \cosh ^{2}(\sqrt{c} y)=c, \quad b>0>a, \quad c=b-a$;

(7) $a \sinh ^{2}(\sqrt{c} x)-b \sinh ^{2}(\sqrt{c} y)=c, \quad 0>b>a, \quad c=b-a$;

(8) $a \sin ^{2}(\sqrt{c} x)-b \sin ^{2}(\sqrt{c} y)=c, \quad 0>a>b, \quad c=a-b$;

(9) $[\cos (\sqrt{2 b}(x+y))-d] \cdot[\cosh (\sqrt{2 b}(x-y))+d]=c^{2}, \quad b>0, \quad c=\frac{b}{\sqrt{a^{2}+b^{2}}}$, $d=\frac{a}{\sqrt{a^{2}+b^{2}}}$;

$d=\frac{a}{\sqrt{a^{2}+b^{2}}}$;

(10) $[\cosh (\sqrt{-2 b}(x+y))+d] \cdot[\cos (\sqrt{-2 b}(x-y))-d]=c^{2}, \quad b<0, \quad c=\frac{b}{\sqrt{a^{2}+b^{2}}}$,
(11) $e^{x}=\frac{1}{2 \sqrt{y}}$
(12) $y=c \tanh x, \quad c<0$;
(13) $y=c \operatorname{coth} x, \quad c>0$.

Voor het bewijs verwijzen we naar de Engelstalige versie. 
Het begrip deelvariëteit van eindig type is gedefinieerd en uitgewerkt door B.Y. Chen [Ch1] in 1984. Een deelvariëteit $M^{n}$ gelegen in een Euclidische ruimte $\mathbb{E}^{n+p}$ is van eindig type, als en slechts als elke component van het positievectorveld $X$ kan geschreven worden als een eindige som van eigenfuncties van de Laplaciaan $\Delta$ van $M^{n}$. Dit betekent dat

$$
X=X_{0}+\sum_{i=1}^{k} X_{i}
$$

met $X_{0}$ een constante vector en $\Delta X=\lambda_{i} X_{i}$ voor elke $i=1, . ., k$ met $\lambda_{i} \in \mathbb{R}$. Als alle eigenwaarden $\left\{\lambda_{1}, \lambda_{2}, . ., \lambda_{k}\right\}$ verschillend zijn , dan is $M^{n}$ van type $k$. Als één van de $\lambda$ 's nul is, dan is $M^{n}$ van type nul $k$.

Krommen van eindig type in de Euclidische ruimte werden reeds grondig bestudeerd [Ch1],[CDDVV],[CDV]. Eén van de mooie eigenschappen is dat een gesloten kromme $\alpha(s)$ in $\mathbb{E}^{m}$ eindig Chen type heeft als en slechts als de Fourierreeksontwikkeling van de periodische coördinaatsfuncties van $\alpha(s)$ ten opzichte van de booglengteparameter $s$ eindig is.

Verder werd reeds bewezen dat cirkels de enige gesloten krommen van type 1 zijn in $\mathbb{E}^{m}$, de enige niet gesloten krommen van type 1 zijn rechten. Bovendien bewees B.y. Chen dat een gesloten kromme van eindig type gelegen in een Euclidisch vlak $\mathbb{E}^{2}$ een cirkel moet zijn [Ch1]. De enige niet gesloten vlakke kromme van eindig type is de rechte [CDVV]. In hoger dimensionale Euclidische ruimten ziet de situatie er helemaal anders uit. Bijvoorbeeld, in de drie-dimensionale Euclidische ruimte $\mathbb{E}^{3}$ bestaat er voor elke $k \in \mathbb{N}$ een gesloten kromme van type $k$ [CDDVV]. Een volledige classificatie van alle gesloten type 2 krommen in $\mathbb{E}^{m}$ werd beschreven in [CDV]. Bovendien werd in [DPVV] bewezen dat alle type 2 krommen in $\mathbb{E}^{m}$ ofwel gesloten zijn, ofwel W-krommen zijn (= krommen met constante Frenet krommingen).

Beschouw een kromme $\alpha(s)$ gelegen in de n-dimensionale Minkowskiruimte $\mathbb{E}_{1}^{n}$, geparameteriseerd door de booglengteparameter $s$ (we sluiten nul krommen uit). De Laplace operator $\Delta$ van $\alpha(s)$ is dan gelijk aan

$$
\Delta= \pm \frac{d^{2}}{d s^{2}}
$$

De eigenfuncties van $\Delta$ zijn $\cos (a s), \sin (a s), \cosh (a s), \sinh (a s)$ en $s$. Analoog met Chens definitie, bekomen we dat een kromme $\alpha(s)$ in $\mathbb{E}_{1}^{n}$ van eindig type kan geschreven worden als

$$
\alpha(s)=a_{0}+b_{0} s+\sum_{t=1}^{k_{1}}\left[a_{t} \cos \left(p_{t} s\right)+b_{t} \sin \left(p_{t} s\right)\right]+\sum_{t=1}^{k_{2}}\left[c_{t} \cosh \left(q_{t} s\right)+d_{t} \sinh \left(q_{t} s\right)\right]
$$

met $a_{i}, b_{i} \in \mathbb{R}^{n} ; \quad 0<p_{1}<p_{2}<. .<p_{k_{1}} \quad$ en $\quad 0<q_{1}<q_{2}<. .<q_{k_{2}}$.

We gaan op zoek naar de krommen van eindig type in $\mathbb{E}_{1}^{2}$, en bekomen een resultaat dat vrij parallel loopt met het Euclidische geval. 
Stelling 6. Elke kromme van eindig type in $\mathbb{E}_{1}^{2}$ is van type 1 , en zodoende een deel van een orthogonale hyperbool of een deel van een rechte.

De classificatie van alle krommen van type 2 in $\mathbb{E}_{1}^{3}$ is onze volgende doelstelling. Sommige van deze krommen zijn gelegen in een vlak, maar dan wel in een isotropisch vlak.

Stelling 7. Een vlakke kromme van type 2, gelegen in een isotropisch vlak van $\mathbb{E}_{1}^{3}$, is een ruimtelijke kromme van type nul 2.

Omdat het aantal niet-vlakke krommen van Chen-type 2 in $\mathbb{E}_{1}^{3}$ nogal omvangrijk is, bekijken we eerst de krommen van nul 2-type.

Stelling 8. Een niet-vlakke kromme $\alpha(s)$ in $\mathbb{E}_{1}^{3}$ is een nul 2-type kromme als en slechts als $\alpha(s)$ een deel is van één van de volgende krommen :

$$
\begin{array}{cll}
\alpha(s)=(a s, b \cos s, b \sin s), & a, b \in \mathbb{R}_{0} ; & |a| \neq|b| ; \\
\alpha(s)=(a \cosh s, a \sinh s, b s), & a, b \in \mathbb{R}_{0} ; \quad|a| \neq|b| ; \\
\alpha(s)=(a \sinh s, a \cosh s, b s), & a, b \in \mathbb{R}_{0} ; \quad|a| \neq|b| .
\end{array}
$$

Merk op dat al deze krommen gelegen zijn op een cylinder; (1) op een circulaire cylinder, (2) en (3) op een hyperbolische cylinder. Kromme (2) is een ruimtelijke kromme; het causaal karakter van kromme (1) en (3) is afhankelijk van de gekozen constanten.

We bestuderen nu de krommen van Chen-type 2 waarvan de beide eigenwaarden verschillen van nul.

Stelling 9. Een niet-vlakke kromme $\alpha(s)$ in $\mathbb{E}_{1}^{3}$ is van type 2 met beide eigenwaarden verschillend van nul als en slechts als $\alpha(s)$ een deel is van één van de volgende krommen :

$$
\begin{gathered}
\alpha(s)=(\rho \sin s, \epsilon \cos s+a \cos (3 s), \epsilon \sin s+a \sin (3 s)), \quad \rho^{2}-12 a \epsilon=0 ; \quad \rho, \epsilon, a \in \mathbb{R}_{0} \\
\alpha(s)=\left(a \cosh s+\lambda b \sinh s-4 c e^{(3 \lambda s)},-b \cosh s-\lambda a \sinh s+4 c e^{(3 \lambda s)}, 2 d e^{\lambda s}\right) \\
d^{2}-6(a-b) c=0, \quad a, b, c, d \in \mathbb{R}_{0}, \quad \lambda \in\{-1,1\} \\
\alpha(s)=\left(a e^{s}+b \cosh (3 s), a e^{s}+b \sinh (3 s), c e^{-s}\right) ; \\
c^{2}+6 a b=0, \quad a, b, c \in \mathbb{R}_{0} ;
\end{gathered}
$$

$\alpha(s)=(\epsilon \cosh s+a \cosh (3 s), \epsilon \sinh s+a \sinh (3 s), \rho \cosh s) ; \quad \rho^{2}+12 a \epsilon=0 ; \quad \rho, \epsilon, a \in \mathbb{R}_{0} ;$ $\alpha(s)=(\epsilon \cosh s+a \cosh (3 s), \epsilon \sinh s+a \sinh (3 s), \rho \sinh s) ; \quad \rho^{2}+12 a \epsilon=0 ; \quad \rho, \epsilon, a \in \mathbb{R}_{0} ;$

$$
\begin{gathered}
\alpha(s)=\left(a e^{s}+b \sinh (3 s), a e^{s}+b \cosh (3 s), c e^{-s}\right) \\
c^{2}-6 a b=0, \quad a, b, c \in \mathbb{R}_{0}
\end{gathered}
$$


$\alpha(s)=(\epsilon \sinh s+a \sinh (3 s), \epsilon \cosh s+a \cosh (3 s), \rho \cosh s) ; \quad \rho^{2}-12 a \epsilon=0 ; \quad \rho, \epsilon, a \in \mathbb{R}_{0} ;$

$\alpha(s)=(\epsilon \sinh s+a \sinh (3 s), \epsilon \cosh s+a \cosh (3 s), \rho \sinh s) ; \quad \rho^{2}-12 a \epsilon=0 ; \quad \rho, \epsilon, a \in \mathbb{R}_{0}$.

Merk op dat al deze krommen gelegen zijn op een kwadriek, (1) op een elipsoïde en de andere op een hyperboloïde.

* De krommen (1), (3), (4) en (5) zijn ruimtelijke krommen; krommen (6), (7) en (8) zijn Lorentz krommen. Het causaal karakter van de andere krommen is afhankelijk van de keuze van de constanten. 


\section{§4. MINIMALE OPPERVLAKKEN}

Minimale oppervlakken en minimale deelvariëteiten staan, ondanks het feit dat het begrip al "lang" bekend is, nog steeds in de belangstelling. De lezer kent allicht in deze context het probleem van de fysicus J. Plateau, die rond 1850 experimenteel tot de bevinding kwam dat minimale oppervlakken kunnen geconstrueerd worden met een gegeven gesloten ruimtekromme als rand (zeepbellenprobleem).

Een oppervlak $M$ in $\mathbb{E}_{1}^{n}$ is minimaal als en slechts als het gemiddeld krommingsvectorveld gelijk is aan nul, dus

$$
H=0
$$

of, nog anders geformuleerd als

$$
\operatorname{tr} A_{\xi_{i}}=0, \text { voor elke } i=3, \ldots, n .
$$

Deze voorwaarde komt overéén met het gelijkstellen aan nul van de eerste variatie van de oppervlaktefunctionaal.

Historisch gezien, zijn de drie eerste voorbeelden van niet-triviale minimale oppervlakken in de 3-dimensionale Euclidische ruimte $\mathbb{E}^{3}$ de catenoïde, de helicoïde en de minimale translatieoppervlakken. De catenoïden zijn (vlakken niet in beschouwing genomen ) de enige minimale omwentelingsoppervlakken (Euler 1744, Meusnier 1776). Deze oppervlakken bekomt men door een kettinglijn ("catenary") te wentelen rond de z-as. De helicoïden zijn (op vlakken na ) de enige minimale regeloppervlakken (Meusnier 1776, Catalan 1844). De minimale translatieoppervlakken werden ontdekt door Scherk in 1835; hij bewees dat (buiten vlakken) de oppervlakken met de volgende vergelijking

$$
z=\log \left|\frac{\cos y}{\cos x}\right|
$$

de enige minimale translatieoppervlakken zijn. Van alle andere minimale oppervlakken, is dat van Enneper het best gekende [Sp].

De minimale oppervlakken in de 3-dimensionale Minkowskiruimte $\mathbb{E}_{1}^{3}$ met metriek $g=d x^{2}+d y^{2}-d z^{2}$ werden in 1983 bestudeerd door Kobayashi. Hij gaf een classificatie van alle ruimtelijke minimale - hijzelf noemde ze "maximale" omdat de tweede variatie van de oppervlaktefunctionaal steeds negatief definiet is voor ruimtelijke oppervlakken in $\mathbb{E}_{1}^{3}$ - omwentelingsoppervlakken en regeloppervlakken. Van de Woestyne classificieerde in 1989 alle Lorentz minimale omwentelingsoppervlakken en Lorentz regeloppervlakken in $\mathbb{E}_{1}^{3}$. Bovendien vond hij alle minimale translatieoppervlakken in $\mathbb{E}_{1}^{3}$ voorzien van de metriek $g=d x^{2}+d y^{2}-d z^{2}$.

We geven de bekomen resultaten zonder bewijs, voor de bewijzen verwijzen we naar [Ko] en [VdW1].

Stelling 10. Elk minimaal ruimtelijk translatieoppervlak in $\mathbb{E}_{1}^{3}$ met metriek $g=$ $d x^{2}+d y^{2}-d z^{2}$, is congruent met een deel van één van de volgende oppervlakken :

i. een ruimtelijk vlak,

ii. het oppervlak van Scherk van de eerste soort. 
Stelling 11. Elk minimaal Lorentz translatieoppervlak in $\mathbb{E}_{1}^{3}$ met metriek $g=d x^{2}+$ $d y^{2}-d z^{2}$, is congruent met een deel van één van de volgende oppervlakken:

i. een Lorentz vlak,

ii. het oppervlak van Scherk van de eerste soort,

iii. het oppervlak van Scherk van de tweede soort,

iv. het oppervlak van Scherk van de derde soort,

v. een vlakke B-scroll over een nul kromme,

$$
z(x, y)= \pm x+g(y)
$$

met $g(y)$ een willekeurige functie.

De ruimtelijke minimale oppervlakken in $\mathbb{E}_{1}^{3}$ worden bepaald in de volgende stelling.

Stelling 12. Elk minimaal ruimtelijk regeloppervlak in $\mathbb{E}_{1}^{3}$ is congruent met een deel van één van de volgende oppervlakken:

i. een ruimtelijk vlak;

ii. de helicoïde van de eerste soort;

iii. de helicoïde van de tweede soort;

iv. het toegevoegde oppervlak van Enneper van de tweede soort.

Een classificatie van de Lorentz minimale oppervlakken in $\mathbb{E}_{1}^{3}$ wordt gegeven in de volgende stelling.

Stelling 13. Elk minimaal Lorentz regeloppervlak in $\mathbb{E}_{1}^{3}$ is congruent met een deel van één van de volgende oppervlakken:

i. een Lorentz vlak;

ii. de helicoïde van de eerste soort;

iii. de helicoilde van de tweede soort;

iv. de helicoïde van de derde soort;

v. het toegevoegde oppervlak van Enneper van de tweede soort;

vi. een vlakke B-scroll over een nul kromme.

We geven nu een classificatie van alle minimale translatieoppervlakken in de $\mathrm{n}$ dimensionale Minkowskiruimte $\mathbb{E}_{1}^{n}$, met metriek, $g=-d x_{1}^{2}+\sum_{i=2}^{n} d x_{i}^{2}$. De gebruikte metriek speelt een belangrijke rol, dit hangt samen met de definitie van een translatieoppervlak. Daarom herhalen we kort wat we juist bedoelen met een translatieoppervlak.

Een translatieoppervlak $M$ in $\mathbb{E}_{1}^{n}$ is een oppervlak beschreven door een immersie

$$
X: U \subset \mathbb{E}^{2} \rightarrow \mathbb{E}_{1}^{n}:(x, y) \rightarrow\left(x, y, f_{3}(x)+g_{3}(y), \ldots, f_{n}(x)+g_{n}(y)\right) .
$$

De volgende stelling geeft de classificatie van minimale translatieoppervlakken in $\mathbb{E}_{1}^{n}$ met de gegeven metriek $g$. 
Stelling 14. $M$ is een minimaal translatieoppervlak in $\mathbb{E}_{1}^{n}$, met metriek $g=-d x_{1}^{2}+$ $\sum_{i=2}^{n} d x_{i}^{2}$, als en slechts als het congruent is met een deel van één van de volgende oppervlakken,

(1) een vlak;

(2) een veralgemeende $B$-scroll;

(3) een oppervlak met parameterisatie

$$
F(x, y)=\left(x, y, f_{3}(x)+g_{3}(y), \ldots, f_{n}(x)+g_{n}(y)\right),
$$

met $\forall i \in\{3, . ., n\}$,

$$
f_{i}(x)=\frac{-c_{i}}{A} \log |A x|+\left(k_{i}-c_{i} \frac{B}{A}\right) x ;
$$

of

$$
f_{i}(x)=\frac{-c_{i}}{A} \log \mid \cos \left(\sqrt{A K-B^{2}}(x+l) \mid+\left(k_{i}-c_{i} \frac{B}{A}\right) x\right.
$$

of

$$
f_{i}(x)=\frac{-c_{i}}{A} \log \left(\cosh \left(\sqrt{B^{2}-A K} x\right)\right)+\left(k_{i}-c_{i} \frac{B}{A}\right) x
$$

of

$$
f_{i}(x)=\frac{-c_{i}}{A} \log \left|\sinh \left(\sqrt{B^{2}-A K} x\right)\right|+\left(k_{i}-c_{i} \frac{B}{A}\right) x
$$

en

$$
g_{i}(y)=\frac{c_{i}}{A} \log \left|\cos \left(\sqrt{A L-\tilde{B}^{2}} y\right)\right|+\left(l_{i}-c_{i} \frac{\tilde{B}}{A}\right) y
$$

met

$$
\begin{gathered}
A=\sum_{j=3}^{n} c_{j}^{2}, \quad B=\sum_{j=4}^{n} c_{j} k_{j}, \quad K=-1+\sum_{j=4}^{n} k_{j}^{2}, \\
\tilde{B}=\sum_{j=4}^{n} c_{j} l_{j}, \quad L=1+\sum_{j=4}^{n} l_{j}^{2} .
\end{gathered}
$$

Voor $n=3$ geeft dit

Stelling 15. $M$ is een minimaal ruimtelijk translatieoppervlak in $\mathbb{E}_{1}^{3}$, met metriek $g=-d x_{1}^{2}+d x_{2}^{2}+d x_{3}^{2}$, als en slechts als het congruent is met een deel van één van de volgende oppervlakken,

(1) een ruimtelijk vlak;

(2) een oppervlak met parameterisatie

$$
z(x, y)=\frac{1}{a} \log \left|\frac{\cos (a y)}{\sinh (a x)}\right|,
$$

met $a \in \mathbb{R}_{0}, \quad \operatorname{coth}^{2}(a x)-\tan ^{2}(a y)>1$. 
Stelling 16. $M$ is een minimaal Lorentz translatieoppervlak in $\mathbb{E}_{1}^{3}$, met metriek $g=-d x_{1}^{2}+d x_{2}^{2}+d x_{3}^{2}$, als en slechts als het congruent is met een deel van één van de volgende oppervlakken,

(1) een Lorentz vlak;

(2) een vlakke B-scroll;

$$
z(x, y)= \pm x+g(y)
$$

met $g(y)$ een willekeurige functie;

(3) een oppervlak geparameteriseerd door

$$
z(x, y)=\frac{1}{a} \log \left|\frac{\cos (a y)}{\cosh (a x)}\right|,
$$

met $a \in \mathbb{R}_{0}$;

(4) een oppervlak met parameterisatie

$$
z(x, y)=\frac{1}{a} \log \left|\frac{\cos (a y)}{\sinh (a x)}\right|,
$$

met $a \in \mathbb{R}_{0}, \quad \operatorname{coth}^{2}(a x)-\tan ^{2}(a y)<1$. 


\section{§5. REGELOPPERVLAKKEN VAN EINDIG TYPE}

Zoals we reeds zagen in de vorige paragraaf, bewees Catalan dat vlakken en helicoïden de enige minimale regeloppervlakken zijn in de drie-dimensionale Euclidische ruimte. Minimale deelvariëteiten, dus ook oppervlakken, zijn een deel van een veel grotere klasse van speciale deelvariëteiten, namelijk de deelvariëteiten van eindig type.

In [CDVV] werden de regeloppervlakken van eindig type in de Euclidische ruimte $\mathbb{E}^{n}$ bestudeerd, dit resulteerde in de volgende stellingen.

Stelling 16. Een regeloppervlak $M$ in $\mathbb{E}^{n}$ is van eindig type als en slechts als $M$ een cylinder over een kromme van eindig type is of $M$ een deel is van een helicoïde in een drie-dimensionale deelruimte $\mathbb{E}^{3}$.

Als we deze stelling bekijken voor $n=3$, en wetend dat de krommen van eindig type in $\mathbb{E}^{2}$ rechten en cirkels zijn, dan bekomen we de volgende stelling.

Stelling 17. Een regeloppervlak $M$ in $\mathbb{E}^{3}$ is van eindig type als en slechts als $M$ een deel is van een vlak, of van circulaire cylinder of van een helicoïde.

In [D] werden alle regeldeelvariëteiten van eindig type gelegen in een Euclidische ruimte als volgt geclassificieerd.

Stelling 18. Een regeldeelvariëteit $M^{n+1}$ in $\mathbb{E}^{n+p}$ is van eindig type als en slechts als $M^{n+1}$ een deel is van een cylinder over een kromme van eindig type of van een veralgemeende helicoïde.

De minimale regeloppervlakken in $\mathbb{E}_{1}^{3}$ zijn een deelverzameling van alle regeloppervlakken van eindig type in $\mathbb{E}_{1}^{3}$. We gaan dus na of er niet-minimale regeloppervlakken van eindig type bestaan in de drie-dimensionale Minkowskiruimte.

Stelling 19. Een regeloppervlak $M$ in $\mathbb{E}_{1}^{3}$ is van eindig type als en slechts als

(1) $M$ is minimaal, of

(2) $M$ is een deel van een circulaire cylinder, of

(3) $M$ is een deel van een hyperbolische cylinder, of

(4) $M$ is een isoparametrisch oppervlak met nul regels.

De gevallen (2) en (3) zijn van nul 2-type; het laatste geval is ook van nul 2-type, behalve wanneer het een éénbladige hyperboloïde is, want dat heeft type 1 . We bekijken de regeloppervlakken met nul regels eens van naderbij.

Stelling 20. Stel dat $M$ een niet cylindrisch regeloppervlak is in $\mathbb{E}_{1}^{3}$ met nul regels, dan zijn de volgende eigenschappen equivalent,

(1) $\frac{g(\dot{\beta}, \dot{\beta})}{g(\dot{\alpha}, \beta)^{2}}=$ constante verschillend van nul,

(2) de Gausskromming $K$ van $M$ is constant,

(3) de gemiddelde kromming $H$ van $M$ is constant,

(4) $M$ is isoparametrisch (zoals beschreven in [M]). 
Zoals we reeds opmerkten, is de éénbladige hyperboloïde een regeloppervlak van eindig type met nul regels. Inderdaad, als we er vanuit gaan dat $g(\alpha, \beta)=0$ ( merk op dat deze voorwaarde onafhankelijk is van de keuze van $\alpha$ of $\beta$ ), dan kunnen we aantonen dat

$$
\alpha=b \beta+\frac{g(\alpha, \dot{\beta})}{g(\dot{\beta}, \dot{\beta})} \dot{\beta},
$$

met $b$ een willekeurige functie van $s$. Dan is

$$
X(s, t)=\frac{g(\alpha, \dot{\beta})}{g(\dot{\beta}, \dot{\beta})} \dot{\beta}+(b+t) \beta,
$$

en

$$
g(X, X)=\frac{1}{\rho}
$$

Als $\rho$ constant is, of dus $M$ van eindig type is, bekomen we de éénbladige hyperboloïde.

Er bestaat een procedure om lokaal alle regeloppervlakken van eindig type met nul regels te genereren. Merk op dat $\beta$ altijd lokaal kan geherparameteriseerd worden als

$$
\beta(s)=(1, \cos (s), \sin (s)) .
$$

Dan is $g(\dot{\beta}(s), \dot{\beta}(s))=1$ en $\alpha$ moet voldoen aan

$$
g(\dot{\alpha}(s), \beta(s))=c
$$

met $c$ een constante verschillend van nul.

Stel $\alpha(s)=\left(\alpha_{1}(s), \alpha_{2}(s), \alpha_{3}(s)\right)$, dan wordt de bovenstaande voorwaarde

$$
\dot{\alpha}_{1}=-c+\dot{\alpha}_{2}(s) \cos (s)+\dot{\alpha}_{3}(s) \sin (s) .
$$

We kunnen, zonder afbreuk te doen aan de algemeenheid, veronderstellen dat $\alpha$ gelegen is in het vlak $x_{3}=0$. Als $\alpha_{2}(s)=f(s)$, dan wordt het oppervlak beschreven door

$$
X(s, t)=\left(-c s+\int f^{\prime}(s) \cos (s) d s+t, f(s)+t \cos (s), t \sin (s)\right) .
$$

Dit is, lokaal, de meest algemene vorm van een regeloppervlak van eindig type met niet-parallelle nul regels.

Aan de hand van de bekomen parameterisatie, berekenen we de Laplaciaan $\Delta$,

$$
\Delta=\frac{1}{c^{2}}\left(2 c \frac{\partial^{2}}{\partial s \partial t}-\frac{\partial q}{\partial t} \frac{\partial}{\partial t}-q \frac{\partial^{2}}{\partial t^{2}}\right)
$$

met

$$
q=\left(-c^{2}+\dot{f}^{2} \sin ^{2}(s)+2 c \dot{f} \cos (s)\right)-2 \dot{f} \sin (s) t+t^{2} .
$$

Om het type van deze oppervlakken te kennen, berekenen we

$$
\Delta X(s, t)=\lambda(-\dot{f} \sin (s)+t, c \sin (s)-\dot{f} \sin (s) \cos (s)+\cos (s) t,
$$




$$
\left.-c \cos (s)-\dot{f} \sin ^{2}(s)+\sin (s) t\right)
$$

met $\lambda=\frac{-2}{c^{2}} \in \mathbb{R}_{0}$.

Er zijn nu twee gevallen :

Het eerste geval is dat

$$
\Delta X(s, t)=\lambda X(s, t) .
$$

Dit betekent dat het oppervlak $M$ van type 1 is. In dit geval moet er onder andere voldaan zijn aan

$$
-c \cos (s)-\dot{f} \sin ^{2}(s)=0 .
$$

$\mathrm{Na}$ integratie en door gebruik te maken van de overige voorwaarden, zien we dat

$$
f(s)=\frac{c}{\sin (s)}
$$

Wat de volgende parameterisatie geeft $\operatorname{van} M$,

$$
X(s, t)=\left(c \cot (s)+t, \frac{c}{\sin (s)}+t \cos (s), t \sin (s)\right) \text {. }
$$

Dit is een niet alledaagse parameterisatie van de hyperbolö̈de.

In het tweede geval is

$$
\begin{gathered}
\Delta X(s, t)=\lambda X_{2}(s, t), \\
X_{2}(s, t) \neq X(s, t) .
\end{gathered}
$$

Men kan eenvoudig nagaan dat

$$
X(s, t)=X_{1}(s, t)+X_{2}(s, t)
$$

met

$$
X_{1}(s, t)=\left(-c s+\dot{f} \sin (s)+\int \dot{f} \cos (s) d s,-c \sin (s)+\dot{f} \sin (s)+f, c \cos (s)+\dot{f} \sin ^{2}(s)\right) .
$$

Daar $X_{1}$ enkel een functie is van $s$, hebben we dat

$$
\Delta X_{1}=0 .
$$

$X_{1}$ is dus een niet-constante functie, wat betekent dat in dit geval $M$ van type nul 2 is.

Door een speciale keuze van $f(s)$ bekomen we enkele mooie, eenvoudige voorbeelden: i) $f(s)=0$, geeft

$$
X(s, t)=(t-c s, t \cos (s), t \sin (s)) \text {. }
$$

ii) $f(s)=s$, geeft

$$
X(s, t)=(t-c s+\sin (s), s+t \cos (s), t \sin (s)) \text {. }
$$

en tenslotte 
iii) $f(s)=\sin (s)$, geeft

$$
X(s, t)=\left(t-c s+\frac{1}{2} \sin ^{2}(s),(t-1) \cos (s), t \sin (s)\right) .
$$

Minimale deelvariëteiten zijn niet alleen een deel van de deelvariëteiten van eindig type, maar ook van de deelvariëteiten met constante gemiddelde kromming.

Een oppervlak $M$ in $\mathbb{E}^{3}$ heeft constante gemiddelde kromming als

$$
g(H, H)=c,
$$

met $c \in \mathbb{R}, g$ de standaard metriek in $\mathbb{E}^{3}$ en $H$ het gemiddeld krommingsvectorveld. Als $c=0$, dan is $H=0$, dus $M$ is een minimaal oppervlak. De volledige classificatie in $\mathbb{E}^{3}$ ziet er als volgt uit.

Stelling 21. Een regeloppervlak met constante gemiddelde kromming in $\mathbb{E}^{3}$ is een minimaal oppervlak of een circulaire cylinder.

Merk op dat elk regeloppervlak met constante gemiddelde kromming in $\mathbb{E}^{3}$ van eindig type is, en vice versa.

We bestuderen de regeloppervlakken met constante gemiddelde kromming in de 3dimensionale Minkowskiruimte. Vooral de niet-minimale regeloppervlakken zullen uiteraard onze aandacht krijgen.

Stelling 22. Een regeloppervlak $M$ in $\mathbb{E}_{1}^{3}$ heeft constante gemiddelde kromming als en slechts als

(1) $M$ is minimaal, of

(2) $M$ is een deel van een circulaire cylinder, of

(3) $M$ is een deel van een hyperbolische cylinder, of

(4) $M$ is een isoparametrisch oppervlak met nul regels.

Merk op dat ook in het Minkowski geval, elk regeloppervlak met constante gemiddelde kromming van eindig type is en vice versa. 


\section{§6. TENSOR PRODUCT OPPERVLAKKEN}

De studie van tensor product deelvariëteiten is ontstaan uit het onderzoek naar minimale deelvariëteiten in sferen. Het gekende theorema van T. Takahashi [Ta] over deelvariëteiten van type 1 in $\mathbb{E}^{m}$, zette vele meetkundigen aan om alle minimale deelvariëteiten in sferen te bestuderen.

Stelling 23. Een deelvariëteit $M^{n}$ van $\mathbb{E}^{m}$ is van type 1 als en slechts als ofwel $M$ een minimale deelvariëteit van de Euclidische ruimte $\mathbb{E}^{m}$ is, ofwel $M$ een minimale deelvariëteit van een hypersfeer $S^{m-1}$ in $\mathbb{E}^{m}$ is.

De klasse van alle minimale deelvariëteiten in sferen is zeer omvangrijk. Dat is ook de reden dat er bijkomende voorwaarden werden opgelegd om tot concrete resultaten te komen. Eén van deze voorwaarden was dat de kwadratische representatie van zo'n deelvariëteit van eindig type moest zijn. Volgens S.S. Tai [Tai] is de tweede standaard minimale immersie van een sfeer

$$
S^{m}(r)=\left\{x=\left(x_{1}, x_{2}, \ldots, x_{m+1}\right) \in \mathbb{E}^{m+1} \mid x_{1}^{2}+x_{2}^{2}+\ldots+x_{m+1}^{2}=r^{2}\right\}
$$

te schrijven als

$$
\begin{gathered}
f: S^{m}(r) \rightarrow S M(m+1) \\
x \rightarrow x^{t} \cdot x=\left(\begin{array}{c}
x_{1} \\
x_{2} \\
\cdot \\
\cdot \\
x_{m+1}
\end{array}\right) \cdot\left(x_{1}, x_{2}, \ldots, x_{m+1}\right),
\end{gathered}
$$

met $S M(m+1)$ de verzameling van alle reële symmetrische matrices van orde $m+1$, die op een natuurlijke wijze kan geïdentificieerd worden met $\mathbb{R}^{\frac{1}{2}(m+1)(m+2)}$, en zodoende een Euclidische ruimte is met metriek

$$
<A, B>:=\frac{1}{r^{2}} \cdot \operatorname{tr}(A B)
$$

met $A, B \in S M(m+1)$.

Als dan een sferische deelvariëteit gegeven is $x: M^{n} \rightarrow S^{m}(r) \subset \mathbb{E}^{m+1}$, dan is de functie

$$
\phi: M^{n} \stackrel{x}{\longrightarrow} S^{m}(r) \stackrel{f}{\longrightarrow} S M(m+1),
$$

de kwadratische representatie van de deelvariëteit $M^{n}$. Verschillende meetkundigen, waaronder A. Ros [Ros], bestudeerden minimale deelvariëteiten in sferen met een kwadratische representatie van eindig type. Vooral het werk van M. Barros en B.Y. Chen [BC], en van M. Barros en F. Urbano [BU], geeft een overzicht van mooie resultaten in dit domein. 
Stelling 24. Zij $M$ een minimaal oppervlak in een hypersfeer $S^{m}(1)$. Dan is de $k$ wadratische representatie $\phi$ van $M$ :

(1) van type 1 als en slechts als $M$ een totaal geodetische sfeer $S^{2}$ in $S^{3}(1)$ is;

(2) van type 2 als en slechts als $M$ een Veronees oppervlak in $S^{4}(1)$ is of een Clifford torus in $S^{3}(1)$ is;

(3) van type 3 als en slechts als $M$ een Veronees oppervlak in $S^{6}(1)$ is of een equilaterale torus in $S^{5}(1)$ is.

B.Y. Chen bestudeerde de tensor product immersie van twee immersies van een bepaalde Riemannse variëteit. Hij haalde zijn inspiratie in het onderzoek naar de kwadratische representatie van deelvariëteiten. Voor een Euclidische immersie $i: M \rightarrow$ $\mathbb{E}^{m}$ is het tensor product $i \otimes i$ de kwadratische representatie van $M$. Geïnspireerd door Chens aanpak bestudeerden F. Decruyenaere, F. Dillen, L. Vrancken and L. Verstraelen het tensor product van twee immersies van verschillende variëteiten [DDVV].

Definitie 25. [DDVV] Stel dat $M$ en $N$ twee differentieerbare variëteiten van dimensie $r$, resp. $s$ zijn, en veronderstel verder dat

$$
f: M \rightarrow \mathbb{E}^{m}
$$

en

$$
h: N \rightarrow \mathbb{E}^{n}
$$

twee immersies zijn. Dan zijn de directe som en het tensor product als volgt gedefinieerd

$$
f \oplus h: M \times N \rightarrow \mathbb{E}^{m+n}:(p, q) \rightarrow(f(p), h(p))=\left(f^{1}(p), \ldots, f^{m}(p), h^{1}(q), \ldots, h^{n}(q)\right),
$$

en

$f \otimes h: M \times N \rightarrow \mathbb{E}^{m n}:(p, q) \rightarrow f(p) \otimes h(q)=\left(f^{1}(p) h^{1}(q), \ldots, f^{1}(p) h^{n}(q), \ldots, f^{m}(p) h^{n}(q)\right)$

Interessante voorbeelden van tensor product immersies zijn de omwentelingsoppervlakken van Vrănceanu. Deze oppervlakken hebben de volgende parameterisatie

$$
X(u, v)=(r(v) \cos (u) \cos (v), r(v) \cos (u) \sin (v), r(v) \sin (u) \cos (v), r(v) \sin (u) \sin (v))
$$

of als tensor product

$$
X(u, v)=(\cos (u), \sin (u)) \otimes(r(v) \cos (v), r(v) \sin (v)) .
$$

C.S. Houh bewees onder andere dat zo'n oppervlak van eindig type is als en slechts als het een Clifford-torus is, dit is het product van twee vlakke cirkels met dezelfde straal.

De tensor product oppervlakken van twee Euclidische vlakke krommen werden bestudeerd door I. Mihai, R. Rosca, L. Verstraelen en L. Vrancken [MRVV] en door I. Mihai en B. Rouxel [MR].

De tensor product oppervlakken van twee Lorentz krommen staan beschreven in [MVVW] en de bijgevoegde stelling van I.Mihai [M]. 
Wij bestuderen de tensor product oppervlakken afkomstig van een Euclidische kromme en een Lorentz kromme. Het eerste resultaat is de classificatie van alle minimale tensor product oppervlakken.

$\mathrm{Zij} \alpha: \mathbb{R} \rightarrow \mathbb{E}_{1}^{2}(-+)$ en $\beta: \mathbb{R} \rightarrow \mathbb{E}^{2}$ een vlakke Lorentz kromme en een vlakke Euclidische kromme. Stel $\alpha(t)=\left(\alpha_{1}(t), \alpha_{2}(t)\right)$ en $\beta(s)=\left(\beta_{1}(s), \beta_{2}(s)\right)$. Hun tensor product is dan

$$
\begin{gathered}
f=\alpha \otimes \beta: \mathbb{R}^{2} \rightarrow \mathbb{E}_{2}^{4}(--++) \\
f(t, s)=\left(\alpha_{1}(t) \beta_{1}(s), \alpha_{1}(t) \beta_{2}(s), \alpha_{2}(t) \beta_{1}(s), \alpha_{2}(t) \beta_{2}(s)\right) .
\end{gathered}
$$

Als we dan de minimale oppervlakken zoeken, bekomen we het volgende resultaat.

Stelling 26. Als $\alpha: \mathbb{R} \rightarrow \mathbb{E}_{1}^{2}$ een vlakke Lorentz kromme is en $\beta: \mathbb{R} \rightarrow \mathbb{E}^{2}$ een vlakke Euclidische kromme, dan is hun tensor product $\alpha \otimes \beta$ een minimale immersie als en slechts als ofwel:

(1) $\alpha$ of $\beta$ een rechte door de oorsprong is;

(2) $\alpha$ en $\beta$ orthogonale hyperbolen met middelpunt de oorsprong zijn;

(3) $\beta$ een cirkel met middelpunt de oorsprong en $\alpha$ een cirkel of een orthogonale hyperbool met middelpunt de oorsprong is.

We zoeken nu de totally real tensor product oppervlakken. Een totally real deelvariëteit $M$ van een bijna Hermitische variëteit $N$ is een deelvariëteit waarvoor de complexe structuur $J$ van de omvattende variëteit $N$ elke raakvector aan $M$ omzet in een vector gelegen in de normale ruimte van $M$ in $N$. Dit betekent dus dat,

$$
J\left(T_{p} M\right) \subseteq T_{p}^{\perp} M
$$

voor elk punt $p \in M$. Met andere woorden $M$ is een totally real deelvariëteit van $(N, g, J)$ als en slechts als elke raakvector $X$ aan $M$ in om het even welk punt $p \in M$, loodrecht staat op $J X$.

Stelling 27. Het tensor product $\alpha \otimes \beta$ is een totally real immersie ten opzichte van de pseudo-Hermitische structuur $J_{0}$,

$$
J_{0}(u, v, z, w)=(-v, u,-w, z),
$$

op $\mathbb{E}_{2}^{4}$ als en slechts als

(1) $\alpha$ is een orthogonale hyperbool met middelpunt de oorsprong, of

(2) $\beta$ is een rechte door de oorsprong.

Een complexe deelvariëteit $M$ van een bijna Hermitische variëteit $(N, g, J)$ moet voldoen aan de volgende voorwaarde

$$
J\left(T_{p} M\right) \subseteq T_{p} M
$$

voor elk punt $p \in M$. Met andere woorden $M$ is een complexe deelvariëteit $\operatorname{van}(N, g, J)$ als en slechts als voor elke raakvector $X$ aan $M$ in om het even welk punt $p \in M$, de vector $J X$ gelegen is in de rakende ruimte $T_{p} M$. 
Stelling 28. Het tensor product $\alpha \otimes \beta$ is een complexe immersie ten opzichte van de pseudo-Hermitische structuur $J_{0}$,

$$
J_{0}(u, v, z, w)=(-v, u,-w, z),
$$

op $\mathbb{E}_{2}^{4}$ als en slechts als $\alpha$ een rechte is door de oorsprong.

Een deelvariëteit $M$ van een variëteit $N$ is pseudo-minimaal als de lengte van het gemiddeld krommingsvectorveld identiek nul is.

Elke minimale deelvariëteit is ook pseudo-minimaal. Niet-minimale pseudo-minimale deelvariëteiten werden voornamelijk bestudeerd door $R$. Rosca $[R]$. Een classificatie van pseudo-minimale tensor product oppervlakken wordt gegeven in de volgende stelling.

Stelling 29. Het tensor product $\alpha \otimes \beta$ is een niet-minimale pseudo-minimale immersie als en slechts als

$$
\alpha(t)=k e^{ \pm \sqrt{1+b^{2}} t}(\cosh t, \sinh t) \quad, \quad k, b \in \mathbb{R}_{0},
$$

en

$$
\beta(s)=a e^{b s}(\cos s, \sin s) \quad, \quad a>0, \quad b \in \mathbb{R}_{0}
$$

dus $\alpha$ is een hyperbolische spiraal en $\beta$ is een logaritmische spiraal.

Een isometrische immersie $f$ is pseudo-ombilicaal als de shape operator van $H$ een veelvoud is van de identieke functie.

Een equivalente voorwaarde is dat:

$$
<h(X, Y), H\rangle=\lambda\langle X, Y\rangle \quad, \quad X, Y \in \Gamma(T M),
$$

met $\lambda=<H, H>$.

Elke minimale deelvariëteit is pseudo-ombilicaal. Een volledige classificatie van pseudoombilicale tensor producten is niet evident, vandaar dat we enkele deelresultaten vermelden. We bepalen bijvoorbeeld de pseudo-ombilicale tensor product oppervlakken afkomstig van een orthogonale hyperbool met middelpunt de oorsprong in $\mathbb{E}_{1}^{2}$ en een willekeurige kromme in $\mathbb{E}^{2}$.

Stelling 30. Het tensor product $\alpha \otimes \beta$ van een orthogonale hyperbool $\alpha$ met middelpunt de oorsprong in $\mathbb{E}_{1}^{2}$ en een vlakke Euclidische kromme $\beta$ is een niet-minimaal pseudo-ombilicaal oppervlak in $\mathbb{E}_{2}^{4}$ als en slechts als $\beta$ een logaritmische spiraal in $\mathbb{E}^{2}$ is, dus

$$
\beta(s)=k e^{b s}(\cos s, \sin s), \quad k, b \in \mathbb{R}_{0} .
$$

Tenslotte bepalen we pseudo-ombilicale tensor product oppervlakken afkomstig van een willekeurige kromme $\alpha$ in $\mathbb{E}_{1}^{2}$ en een cirkel $\beta$ met middelpunt de oorsprong in $\mathbb{E}^{2}$. We bekomen dat:

Stelling 31. Het tensor product $\alpha \otimes \beta$ van een willekeurige kromme $\alpha$ in $\mathbb{E}_{1}^{2}$ en een cirkel $\beta$ met middelpunt de oorsprong in $\mathbb{E}^{2}$ is een niet-minimaal pseudo-ombilicaal oppervlak in $\mathbb{E}_{2}^{4}$ als en slechts als $\alpha$ een hyperbolische spiraal is, dus

$$
\alpha(t)=k e^{b t}(\cosh t, \sinh t), \quad k, b \in \mathbb{R}_{0} \backslash\{-1,1\} .
$$

